\title{
Geospatial risk analysis of mosquito-borne disease vectors in the Netherlands
}

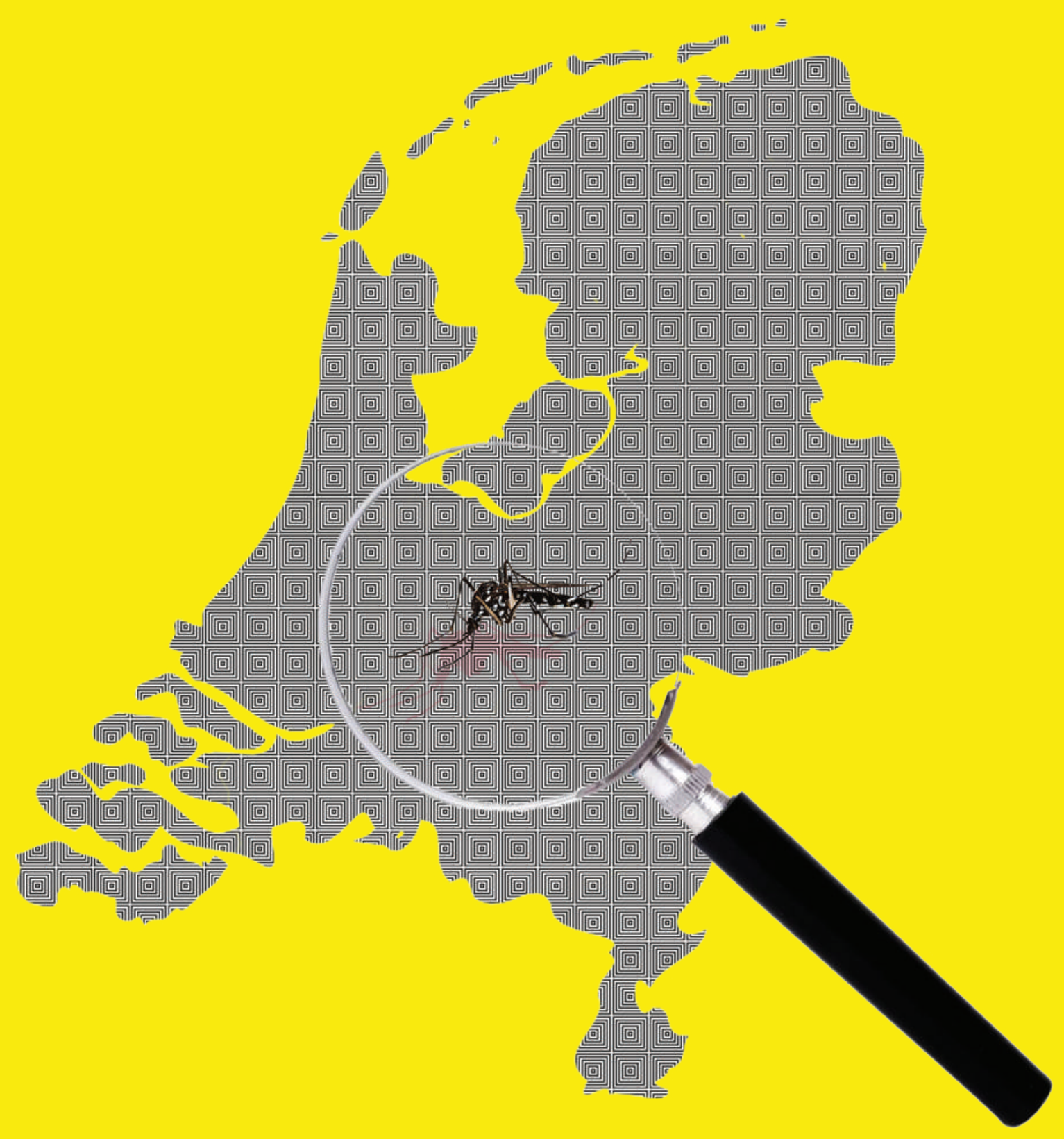







\section{Geospatial risk analysis of mosquito-borne disease vectors in the Netherlands}

Adolfo Ibáñez-Justicia 


\section{Thesis committee}

\section{Promotor}

Prof. Dr W. Takken

Personal chair at the Laboratory of Entomology

Wageningen University \& Research

\section{Co-promotors}

Dr C.J.M. Koenraadt

Associate professor, Laboratory of Entomology

Wageningen University \& Research

Dr R.J.A. van Lammeren

Associate professor, Laboratory of Geo-information Science and Remote Sensing Wageningen University \& Research

\section{Other members}

Prof. Dr G.M.J. Mohren, Wageningen University \& Research

Prof. Dr N. Becker, Heidelberg University, Germany

Prof. Dr J.A. Kortekaas, Wageningen University \& Research

Dr C.B.E.M. Reusken, National Institute for Public Health and the Environment, Bilthoven, The Netherlands

This research was conducted under the auspices of the C.T. de Wit Graduate School for Production Ecology \& Resource Conservation 


\title{
Geospatial risk analysis of mosquito-borne disease vectors in the Netherlands
}

\author{
Adolfo Ibáñez-Justicia
}

Thesis

submitted in fulfilment of the requirements for the degree of doctor at Wageningen University

by the authority of the Rector Magnificus, Prof. Dr A.P.J. Mol, in the presence of the

Thesis Committee appointed by the Academic Board to be defended in public on Friday 1 February 2019 at 4 p.m. in the Aula. 
Adolfo Ibáñez-Justicia

Geospatial risk analysis of mosquito-borne disease vectors in the Netherlands, 254 pages.

$\mathrm{PhD}$ thesis, Wageningen University, Wageningen, the Netherlands (2019) With references, with summary in English

ISBN 978-94-6343-831-5

DOI https://doi.org/10.18174/465838 




\section{Table of contents}

$\begin{array}{lr}\text { Abstract } & 9\end{array}$

$\begin{array}{ll}\text { Chapter } 1 & 11\end{array}$

General introduction

Chapter 2

National Mosquito (Diptera: Culicidae) Survey in The Netherlands 2010-2013

\section{Chapter 3}

Modelling the spatial distribution of the nuisance mosquito species Anopheles plumbeus

(Diptera: Culicidae) in the Netherlands

\section{Chapter 4}

Pathways for introduction and dispersal of invasive mosquito species in Europe: a review

\section{Chapter 5}

Evaluating perceptions of risk in mosquito experts and identifying undocumented pathways for the introduction of invasive mosquito species into Europe

\section{Chapter 6}

Risk-based and adaptive surveillance at Lucky bamboo and used tire importers to prevent the establishment of invasive mosquitoes in the Netherlands

\section{Chapter 7}

The first detected airline introductions of yellow fever mosquitoes (Aedes aegypti) to

Europe, at Schiphol International airport, the Netherlands

\section{Chapter 8}

The effectiveness of Asian bush mosquito (Aedes japonicus japonicus) control actions in colonised peri-urban areas in the Netherlands

\section{Chapter 9}

Habitat suitability modelling to assess the introductions of Aedes albopictus (Diptera,

Culicidae) in the Netherlands

Chapter 10

General discussion

\section{References}

Curriculum vitae 
Abstract: The availability of data on distribution and density of mosquito vectors of disease is needed to understand the risk of mosquito-borne diseases. In case of an outbreak of a newly introduced mosquito-borne pathogen of medical or veterinary importance, such information is required in order to decide on a contingency and eventual control plan. In the recent decade, several exotic mosquito species have become established in European countries and they have rapidly expanded their distribution. The rationale for the present study was the increasingly frequent reporting of invasive mosquito species (IMS) in the Netherlands, some of which are known vectors of infectious diseases, as well as the lack of detailed knowledge on the spatio-temporal distribution of the indigenous mosquito fauna. The aim was therefore to develop methodologies for acquiring accurate information on the actual and potential distribution of indigenous and exotic mosquito species in the Netherlands, and to evaluate the surveillance and control methodologies applied after IMS findings.

To establish a baseline for the spatio-temporal distribution of the indigenous mosquito fauna present in the Netherlands, a survey was conducted at the start of the study. Cross-sectional mosquito field surveys were carried out over a period of four years (2010-2013). These surveys provided occurrence maps for 26 indigenous species. One invasive mosquito species, Aedes japonicus, was discovered using this strategy. Furthermore, data on seasonality of the species, biodiversity and habitat preferences were also provided. Using the collected data on occurrence and abundance, a special study using random forest models was done to investigate the potential spatial distribution and population density of Anopheles plumbeus, a native nuisance mosquito species. I found a high environmental suitability and abundance of this species in the south-eastern provinces, mostly associated with abandoned pig farm buildings, and reports of biting nuisance. The identification of pathways for introduction of IMS was investigated in a next step taking into account the current knowledge and expert opinion. This was done in order to decide on the surveillance strategies needed to reduce the risk of future IMS introductions and/or potential outbreaks of mosquito-borne diseases. Four main routes for IMS introduction and dispersal were identified: the trade in used tires, the import of Lucky bamboo plants from Asia, the passive transport of IMS in vehicles (traffic by road, airplanes, and sea), and the natural dispersal of IMS. The results of the risk-based surveillance of IMS revealed yearly introductions of Ae. albopictus since 2010 at used tire companies and Lucky bamboo greenhouses, sporadic findings of Ae. japonicus associated with used tire trade, the first aircraft associated import of Ae. aegypti in Europe, and the first associated Ae. japonicus import with Lucky bamboo plants from elsewhere in the world. The control of these IMS, implemented after detection, has proven effective to avoid proliferation at these locations and their surroundings. Due to the yearly findings of Ae. albopictus, the potential risk of establishment of this invasive species was further investigated using habitat suitability models. Results show that the current average climatic conditions limit the overwintering of eggs of Ae. albopictus and their survival as adults in many inland areas of the country. However, due to the expected increase of the temperatures in the next decades as a result of climate change, these parts of the Netherlands will offer climatic conditions suitable for sustain populations of this species.

The results presented in this thesis show that nationwide surveillance of mosquitoes is pivotal to gain detailed information on the spatio-temporal distribution and abundance of mosquito species, which is useful to study the habitat suitability of vector species. Furthermore, this thesis highlights the main pathways for introduction and dispersal of IMS, designed a riskbased surveillance of IMS, and evaluated the surveillance and control measures applied in the Netherlands against IMS introductions. The work presented provides essential insights for identifying locations at risk of vector-borne disease transmission, and for designing targeted control of newly introduced IMS in the Netherlands, which is expected in the future. 

Chapter 1

General introduction 


\section{Mosquito-borne diseases in Europe}

Outbreaks of mosquito-borne diseases (MBD) can cause considerable animal and human suffering, and high economic damage (Rich and Wanyoike 2010, Tarantola et al. 2014). With the eradication of dengue and malaria from Europe in the 1950's, the threat from MBD was considered then to be limited only to countries in the tropics. However, during the first years of the 21st century, this situation changed in Europe due to several MBD outbreaks. Some MBD are transmitted by indigenous European mosquito species and are known to occur frequently in Europe, such as Tahyna (Hubalek et al. 2010), tularaemia (Rijks et al. 2013), Sindbis/Ockelbo, Batai, Inkoo and West Nile (Lundstrom 1999). However, in the last years malaria re-emerged in some parts of Europe (Danis et al. 2011), and dengue appeared in France (La Ruche et al. 2010), Croatia (Gjenero-Margan et al. 2010) and Madeira (Sousa et al. 2012). Furthermore, several outbreaks of MBD that originate from (sub)tropical areas also recently occurred in Europe, such as chikungunya in Italy (Rezza et al. 2007, Venturi et al. 2017) and France (Calba et al. 2017), and Usutu outbreaks in Austria (Weissenbock et al. 2003), Italy (Calzolari et al. 2010), Germany (Becker et al. 2012) and The Netherlands (Rijks et al. 2016). Of the above MBD, dengue and chikungunya are exclusively transmitted in Europe by established populations of the invasive mosquitoes species (IMS) Aedes aegypti (Linnaeus) or Ae. albopictus (Skuse) (Medlock et al. 2012). The well-known main driver of these pathogen introductions in the last decades is the accelerating increase in trade and travel (Kilpatrick and Randolph 2012). Nowadays, the growth in air travel has accelerated introductions allowing pathogens to reach other continents within the few days that hosts are infectious, and even during the latent period for some diseases (Kilpatrick et al. 2006). There is concern that other MBD, not indigenous to Europe, might be introduced causing outbreaks, such as Zika, Rift Valley Fever, or Japanese encephalitis. Data from Italy suggest that Japanese encephalitis was already introduced into Europe in 2010 (Ravanini et al. 2012), and until March 2017 more than 2,000 confirmed cases of Zika infections were reported in Europe (Spiteri et al. 2017).

In the European context, the VBORNET and VectorNet projects established a European network of entomological and public health specialists in order to assist the European Centre for Disease Prevention and Control (ECDC) and the European Food Safety Agency (EFSA) in its preparedness activities on vector-borne diseases in the framework of the One-Health concept. These projects have been focused on acquiring a baseline data on confirmed distribution data of major arthropod vectors at various administrative scales. As a result, one 
of the outputs of the projects are the quarterly VectorNet maps showing the current known distribution of vector species in Europe at 'regional' adm inistrative level (NUTS3) (Figure 1; (ECDC \& EFSA 2018).

The aim of this thesis is to develop a methodology to evaluate intervention strategies for the prevention of mosquito borne diseases outbreaks, and includes the implementation of methodologies for knowledge acquisition of the actual and potential spatio-temporal distribution of indigenous and exotic mosquito species.

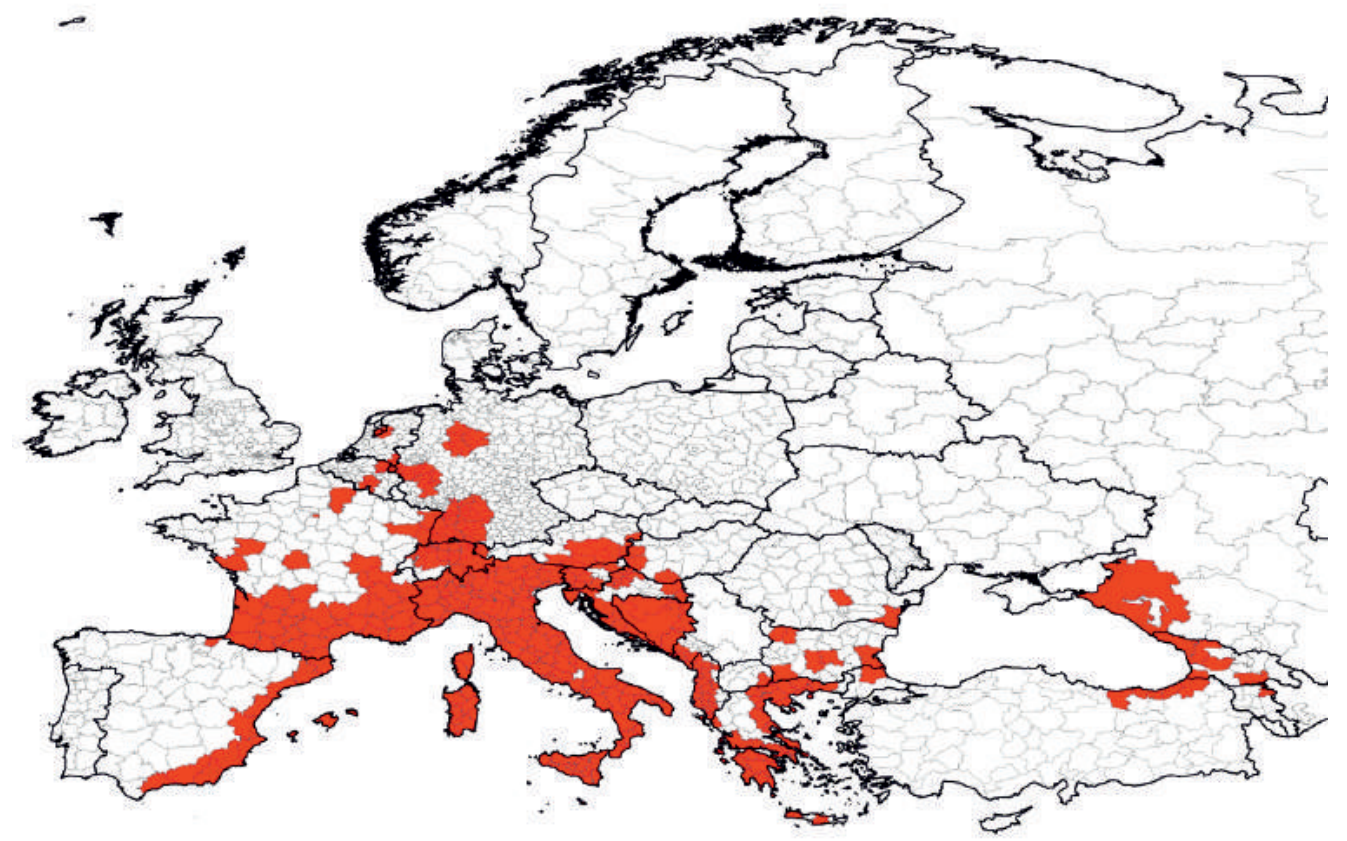

Figure 1. Current known distribution of IMS in Europe at 'regional' administrative level (NUT3). The map is based on confirmed data (published and unpublished) of IMS provided by experts from the respective countries as part of the VBORNET and VectorNet projects. The map includes the areas with Aedes IMS established populations (Ae. albopictus, Ae. aegypti, Ae. japonicus and Ae. koreicus) based on ECDC mosquito maps update June 2018.

\section{Mosquito surveillance}

The collection of spatiotemporal data on mosquito vectors of public health significance is crucial to understand the levels of risk of MBD that the affected countries in Europe face. In case of an outbreak of a new introduced mosquito-borne pathogen of medical or veterinary 
importance, it is necessary to have a distribution map of established populations of all mosquito species (indigenous and exotic) that can serve as vectors of the pathogens, and to know as well when, and roughly in which numbers these vectors occur. Being presence of the mosquito vector a basic requirement for transmission to occur, the higher the abundance of pathogen-infected vectors, the higher will be the risk of disease transmission. Vector abundance is especially important in emerging epidemics because high vector populations (relative to host abundance) result in a high basic reproduction number $\left(\mathrm{R}_{0}\right)$ of the pathogen (Kilpatrick and Randolph 2012). As consequence, a high $\mathrm{R}_{0}$ number will result in higher ability of a disease to persist in a susceptible host population. With the vector information, and information of other factors that can affect the disease transmission (e.g. hosts abundance, environmental conditions), it could be possible to estimate which could be high or low risk areas for a specific disease, which should be the focus areas for the authorities, and where necessary preventive or control measures could and should be taken.

In order to harmonise the mosquito surveillance procedures within the European countries, the European Centre for Disease Prevention and Control (ECDC) produced surveillance guidelines which aim to support the implementation of tailored surveillance for native and invasive mosquito species of public health relevance (Schaffner et al. 2013, ECDC 2014a). In 2010, in the Netherlands, the Centre for Monitoring of Vectors (CMV) of the Netherlands Food and Consumer Product Safety Authority (NVWA) started a risk based and adaptive surveillance of vector species in order to improve the preparedness of the country against vector-borne diseases outbreaks. One of the factors that influenced the establishment of the CMV was the detection of the IMS Ae. albopictus in the summer of 2005 at companies that import Lucky bamboo plants (Dracaena sanderiana) (Scholte et al. 2007). Prior to the start of the CMV monitoring activities, surprisingly little was known about the spatial distribution and life-histories of indigenous mosquito species in the Netherlands, or locations in the country at risk for importing IMS. The Dutch mosquito fauna was then listed in a checklist for dipterans in the Netherlands (Verdonschot 2002), and included 35 indigenous mosquito species. From 2010 onwards, the IMS Ae. albopictus, Ae. aegypti and Ae. atropalpus have been reported from used tire companies (Scholte et al. 2010a, Scholte et al. 2012), and in 2016 Ae. aegypti was reported to be introduced via aircrafts at Schiphol airport (Ibañez-Justicia et al. 2017). Furthermore, established populations of Ae. japonicus have been found in the municipality of Lelystad (Ibañez-Justicia et al. 2014). The introduction and possible establishment of these IMS is a risk for public health due to their ability to transmit vector-borne diseases (VBD): Ae. aegypti is a 
vector in the field of several viruses including yellow fever virus, dengue virus, chikungunya virus and Zika virus, Ae. albopictus is a known field vector of chikungunya virus, dengue virus and dirofilariasis (dog heartworm), laboratory studies have shown the ability of Ae. atropalpus to transmit a number of viruses including La Crosse virus and West Nile virus, and Ae. japonicus has shown to play a role in the transmission of La Crosse virus in the field (Harris et al. 2015, Westby et al. 2015) and under laboratory conditions has shown to be competent for the transmission of a number of viruses including dengue and chikungunya (Schaffner et al. 2011).

\section{Spatio-temporal distribution patterns of mosquito species}

As ectothermal arthropods, mosquito species are unable to regulate their body temperature and are thus sensitive to climate. Climatic conditions influence many important factors in the mosquito life traits such as survival and reproduction rates (Rogers and Randolph 2006), habitat suitability, distribution, abundance, vector activity (particularly biting rates) throughout the year, rates of development, and survival and reproduction of pathogens within vectors (ECDC 2018a). However, climate is only one of many factors influencing vector spatio-temporal distribution. Other factors such as land use, host-preferences, connectivity or vegetation type, can play an important role for the spatio-temporal distribution of mosquito vectors.

For the modelling of the spatial distribution of species, several techniques exist (Elith et al. 2006, Rogers 2006), differing in their assumptions and predictive performance. Species distribution models (SDM) quantitatively describe geographic areas that support the presence of a given species, based on known occurrence data and the associated environmental conditions (Elith and Leathwick 2009). The general idea behind species distribution modelling is to identify relationships between known occurrence of a species (presence/absence) and environmental data (e.g. meteorological data, land use and land cover) and to use these relationships to make predictions for all the unsampled areas in the study region. Among several different spatial distribution modelling techniques for occurrence data of native species, machine learning techniques such as decision trees (e.g., random forests) have been reported to outperform other traditional modelling techniques like linear (and non) discriminant analysis or logistic regression (Cutler et al. 2007, Peters et al. 2007, Cianci et al. 
2015). The insights obtained using random forest modelling has provided more knowledge on vector and non-vector mosquito species distribution (Cianci et al. 2015).

The potential distribution of several Aedes IMS in a new geographical area in Europe has also been assessed through SDM. Using the spatio-temporal distribution and detailed knowledge on the ecological and physiological requirements of the species, in combination with environmental data, habitat suitability maps have been developed to investigate the potential risk of establishment and spread of these invasive species into new areas. For this purpose, several SDM techniques have been employed in Europe such as Geographic Information Systems (GIS) models based on temperature thresholds (Neteler et al. 2011, Roiz et al. 2011, Neteler et al. 2013, Marcantonio et al. 2016), or correlative approaches (Cunze et al. 2016a, Cunze et al. 2016b, Koch et al. 2016). In published SDM for Ae. albopictus (Benedict et al. 2007, ECDC 2009, Kraemer et al. 2015a), the probability of occurrence of the species in the Netherlands is shown as low in comparison with southern European countries. However, to date, this species has been intercepted annually in the Netherlands since 2010 at used tire importers. This raises the question whether the country is suitable for the establishment of this species, and which environmental factors could determine the possible establishment. Local climate conditions can indeed be an important limiting factor for the establishment of $A e$. albopictus after its introduction into 'virgin' territories such as the Netherlands. In previous studies of Takumi et al. (2009), based on the model developed for the UK (Medlock et al. 2006), the potential establishment of Ae. albopictus in the Netherlands was assessed using GIS and climate data (temperature and precipitation) recorded at weather stations. The 2006 climate data set used in the study, was intended to represent the climate experienced by Ae. albopictus following its introduction in the summer of 2005. The study concludes that climate conditions in the Netherlands did not appear to preclude the establishment of the species. However, it was recommended to perform further analysis using long term climate data sets to strengthen the conclusion of the study that the winter conditions in the Netherlands can be permissible for the establishment of temperate strains of Ae albopictus. To date, this analysis has not been done and will be part of this thesis. This analysis will be implemented using data obtained from the Moderate Resolution Imaging Spectroradiometer (MODIS) sensors incorporated in the Terra and Aqua satellites, in order to obtain more spatio-temporal detail regarding micro-climates, especially around cities where warmer temperatures could be expected (Zhao et al. 2014). 


\section{Aim of this thesis}

The aim of this thesis is to develop and implement a methodology to evaluate intervention strategies for the prevention of mosquito borne diseases outbreaks. Such a methodology includes the major goal of knowledge acquisition of the actual and potential spatio-temporal distribution of indigenous and exotic mosquito species.

\section{Outline of this thesis}

Chapter 2 designs the Dutch nationwide mosquito survey with the purpose of providing information about the composition of the indigenous mosquito fauna in the Netherlands. In the study, cross-sectional mosquito field surveys were carried out in more than 700 locations over a period of four years. Based on the National Land Use Map File of The Netherlands, the obtained data were analysed and presented in terms of species composition in three habitat types (urban, agricultural and natural), geographical distribution of the species over the country, and seasonality. Diversity of the three habitats was calculated using richness and evenness indices. The unique value of this study are the generated maps, thereby showing for the first time the current geographical distribution of the native mosquito species. In addition, this survey aims to serve as a tool for discovering undetected IMS established populations in the sampled areas.

Chapter 3 presents habitat suitability and abundance maps for the Netherlands for the mosquito species Anopheles plumbeus, a nuisance species and potential malaria vector. Random forest models were used to link the occurrence and abundance of An. plumbeus with environmental features obtained from satellite imagery and weather. Geospatial mosquito data were provided by the four-year cross-sectional study performed in Chapter 2. For the validation of the occurrence model, data on An. plumbeus presence from confirmed nuisance notifications and data from other mosquito surveys carried out by the CMV during the years 2010-2014 was used.

Chapter 4 and 5 aim to identify and evaluate the pathways for introduction and dispersal of invasive mosquito species in Europe. Identifying and evaluating the importance of these different pathways is key for designing a targeted surveillance strategy that will promptly detect and control introductions of IMS. Chapter 4 presents a review of the pathways for introduction and dispersal of IMS in Europe. The study presented in Chapter $\mathbf{5}$ was set out to identify the 
European mosquito experts' perception of risk for introduction of IMS via different known documented pathways (Chapter 4), to investigate what drives experts' attitudes towards support of taking actions against introduction of IMS and to investigate if other, not previously documented introduction pathways are present. For this purpose, a questionnaire to collect expert data was distributed among participants of the EMCA (European Mosquito Control Association) conference in Montenegro in March 2017.

Chapters 6, 7 and 8 analyze the results of the risk based IMS surveillance, and their management and control in the Netherlands. Chapter 6 presents the results (2010-2016) of a risk-based and adaptive surveillance of IMS implemented in Lucky bamboo greenhouses and used tires companies, with a focus on the effectiveness of the surveillance methodology, management and control operations. In addition, a revised methodology to assign used tire companies to different risk categories for the import of IMS is presented. Chapter $\mathbf{7}$ describes the methodology used for surveillance of IMS at the international airport of Schiphol in the Netherlands, and reports the first detected airline introductions of yellow fever mosquitoes ( $A e$. aegypti) at the airport facilities in 2016. The vector-control response that followed these events was also detailed. Efforts to identify the origin of the introductions, combined data on flight departure locations provided by the airport authorities and genetic assignment tests of the specimens captured. Chapter 8 presents the results of surveillance of the Asian bush mosquito (Ae. japonicus) as well as the control measures applied in colonized peri-urban areas in the Netherlands. The aim of the study was 1) to evaluate the effectiveness of the mosquito control actions conducted in 2015 and 2016 in terms of reducing the populations at the mosquito hotspots (allotment gardens), 2) to identify the most preferred breeding sites in the allotment gardens, 3) to evaluate the reduction of the mosquito populations at the treated allotments compared to the populations in non-infested allotments from adjacent municipalities in the province, and 4) to study the population dynamics of Ae. japonicus through longitudinal mosquito surveillance at the infested locations.

In Chapter 9 I develop, first suitability maps using a species distribution model based on the maximum entropy niche modelling approach (MAXENT) taking into consideration the most updated occurrence data of Ae. albopictus in Europe, and second, an spatial logic conditional model based on the temperature requirements of the species and using Land Surface Temperature data (LST-model). The final aim of the suitability maps is to evaluate the findings of Ae. albopictus obtained in Chapter 6 during the IMS surveillance in used tire companies in order to discern the possibility of overwintering of the species at these locations, or to relate the 
findings to new introductions (e.g. with import of tires). The obtained modelling results aim to help to optimize the efficiency of surveillance and control of IMS programs to contain the possible spread of the species if introduced.

Chapter 10 integrates the obtained results of this thesis into a broader context, by evaluating the factors that influence the acquired knowledge about the spatiotemporal distribution of mosquito species in the Netherlands obtained with the CMV surveillance. In this chapter, results obtained in this thesis will be discussed with a view to obtain a general model on which risk of introduction and establishment, and potential control strategies can be based.

\section{Acknowledgements}

I thank Willem Takken, Sander Koenraadt, Ron van Lammeren and Anton van der Sommen for providing comments on an earlier version of this chapter. 



\section{Chapter 2}

\section{National Mosquito (Diptera: Culicidae) Survey in the Netherlands \\ 2010-2013}

Adolfo Ibáñez-Justicia, Arjan Stroo, Marian Dik, Jacob Beeuwkes \& Ernst-Jan Scholte

Centre for Monitoring of Vectors (CMV), Netherlands Food and Consumer Product Safety Authority (NVWA), Wageningen, The Netherlands

Published in Journal of Medical Entomology (2015) 52(2):185-98 


\begin{abstract}
From 2010 onwards, a nationwide mosquito monitoring scheme has been conducted in the Netherlands with the aim of gaining crucial information about mosquito (Diptera, Culicidae) species composition, geographical distributions, biodiversity and habitat preferences. The results of this study are based on 778 randomly sampled mosquito locations. These are divided into three main habitat types: urban, rural-agricultural and natural areas. Twenty-seven mosquito species were found: 26 indigenous and one exotic, Aedes j. japonicus (Theobald, 1901). The preliminary results are presented here, with details of their species distribution and seasonality. Monitoring the temporal and spatial distribution of mosquitoes is an essential step in the risk analysis of emerging mosquito-borne diseases.
\end{abstract}

Keywords: mosquito, monitoring, vector, nationwide, Netherlands 


\section{Introduction}

Over recent decades, Europe has witnessed several outbreaks of mosquito-borne diseases. Some of these are known to occur frequently in Europe, such as Tahyna virus (Hubalek et al. 2010), Tularaemia (Rijks et al. 2013), Sindbis, Ockelbo, Batai, Inko and West Nile viruses (Lundstrom 1999). Furthermore, some diseases have re-emerged in some parts of Europe, e.g. malaria in Greece (Danis et al. 2011). Since the turn of the century, several outbreaks of mosquito-borne diseases that originated from (sub)tropical areas have also occurred sporadically in Europe. There were major dengue outbreaks in parts of Europe in the past (Reiter 2010), but it has reemerged in France (La Ruche et al. 2010), Croatia (Gjenero-Margan et al. 2010) and Madeira Islands, Portugal (Sousa et al. 2012). Others that have never been reported in Europe before, have been recorded in recent years including chikungunya virus in Italy (Rezza et al. 2007) and France (Gould et al. 2010), and Usutu virus outbreaks in Austria (Weissenbock et al. 2003), Italy (Calzolari et al. 2010), and Germany (Becker et al. 2012). Dengue and chikungunya viruses are transmitted in Europe by the invasive mosquitoes species Aedes aegypti and Aedes albopictus only (Medlock et al. 2012), whereas the other viruses are transmitted by indigenous European mosquitoes. Concern has been raised over the potential for other mosquito-borne diseases, currently not indigenous to Europe, to be introduced, such as Rift Valley Fever virus, or Japanese encephalitis virus. Recent data from Italy suggest that the latter was already introduced into Europe in 2010 (Ravanini et al. 2012). Outbreaks of these diseases can cause considerable animal/human suffering, and high economic impact (Rich and Wanyoike 2010, Tarantola et al. 2014). Furthermore, with regard to the important role as vectors of veterinary and human pathogens, mosquito bites can cause a considerable nuisance for humans and mammals and can have negative economic consequences (e.g. in tourism, work productivity outdoors, meat and dairy production) when mosquitoes attack in large numbers (Connelly and Carlson 2009). Should an outbreak of a mosquito transmitted pathogen of human or veterinary concern (such as Rift Valley fever, West Nile virus, dengue, chikungunya, or Japanese encephalitis) take place in the Netherlands or in a neighboring country, it is necessary to have an understanding of the spatial extent of all mosquito species (indigenous and exotic) that might be vectors of pathogens, with some comparative information on their abundance and seasonal activity. This kind of information is important for estimating levels of risks for a variety of diseases, enabling better targeting for surveillance and control. For this reason, information on mosquito fauna 
composition, potential vector competence and capacity, and their respective geographical distribution is essential to produce disease risk maps.

The Dutch mosquito fauna is listed in a checklist for dipterans in the Netherlands (Verdonschot 2002), and includes 35 indigenous mosquito species. Despite their importance as vectors, surprisingly little has been studied, published or is known about their spatial distribution and lifehistories in the Netherlands. Since the foundation of the Centre for Monitoring of Vectors (CMV) at the end of 2009, the CMV has carried out various surveys focused on vectors (indigenous and exotic) throughout the Netherlands. During exotic mosquito surveys, invasive mosquito species have been routinely found in known risk locations such as used tire storage facilities, Lucky bamboo greenhouses, and airports. Exotic species found were Aedes albopictus (Skuse, 1895), Ae. aegypti (Linnaeus, 1762), Ae. atropalpus (Coquillett, 1902) (Scholte et al. 2007, Scholte et al. 2008, Scholte et al. 2010a, Scholte et al. 2012), Culex quinquefasciatus (Say, 1823), Cx. antennatus (Becker, 1903), and Ae. mcintoshi (Huang, 1985) (Scholte et al. 2010b, Scholte et al. 2014). In other vector surveys, CMV has reported several mosquito species, including Anopheles algeriensis (Theobald, 1903) (Scholte et al. 2011), and the presence of populations of the exotic species Ae.j. japonicus (Theobald, 1901) in the municipality of Lelystad (Ibañez-Justicia et al. 2014).

In order to prepare for the threat of future vector-borne diseases (VBD), CMV started a nationwide inventory of indigenous mosquitoes in 2010 with the aim of gaining crucial information about the current mosquito species composition and its geographical distribution, biodiversity and habitat patterns. This survey was adapted in the Netherlands from the MODIRISK national inventory of mosquitoes in Belgium carried out in 2007 and 2008 (Versteirt et al. 2013). Data collected during the NVS-mosquito survey will also be used to inform statistical vector distribution models. Species distribution modelling combines the presence and/or abundance of species with environmental data in order to identify environmental predictor variables associated with their distribution. Models will provide input for the disease-risk models of The National Institute for Public Health and the Environment (RIVM) and the Central Veterinary Institute (CVI) in the Netherlands. Sam pling protocol and results of four years' mosquito survey (2010-2013) are described in this paper. 


\section{Materials and Methods}

The National Vector Survey (NVS) is nationwide and designed to run for many years. The approach of this survey is to define the mosquito species composition for three habitat types (urban, rural-agriculture, and nature) in the Netherlands. For this purpose, samples of mosquitoes were collected in 778 random locations in the months April until October from 2010 until 2013 (Figure 1).

\section{Sampling design:}

Cross-sectional field surveys were carried out using the National Land Use Map File of The Netherlands (LGN5) as a basis. This LGN5 file, based on satellite images from the years 2003 and 2004, provides 39 land use classes in the Netherlands, from farmland, forests, water, urban areas and nature classes. The 39 land use classes of the raster file were aggregated into three classes: urban, rural-agriculture, and nature. Intensively used agricultural areas such as arable land (e.g. potato or bulb fields), were not included in the rural-agricultural sampling area and considered as unsuitable for the presence of mosquito breeding sites. Using ArcGIS version 9.3, 792 randomly generated sample coordinates (random points) were created from 2010 until 2013 (260 random points in 2010, 225 in 2011, 157 in 2012, and 150 in 2013) in the three defined land use classes in the following proportion: $40 \%$ urban, $40 \%$ rural-agricultural, $20 \%$ natural. Urban areas represent around $20 \%$ of the country total area. Rural-agricultural areas and natural areas represent $60 \%$ and $20 \%$ of the country total area respectively. Urban areas were sampled to a greater extent because of their higher involvement in public health risks caused by mosquito vectors. Each random point was assigned to a full postal address. Fourteen of the planned random points were not sampled during the survey.

\section{Sampling trap:}

In each location, one $\mathrm{CO}_{2}$ baited Mosquito Magnet Liberty Plus trap (Woodstream ${ }^{\circledR}$ Co., Lititz, USA; hereafter MM trap) trap was used. This MM trap uses $\mathrm{CO}_{2}$ and octenol to attract blood seeking mosquitoes, and has been evaluated successfully against a variety of mosquito genera and species for trapping and surveillance (Dennett et al. 2004, McKenzie and Bedard 2004). This trap lures the mosquitoes and other biting insects by producing a continuous stream of $\mathrm{CO}_{2}$, heat and moisture into the air while at the same time the counter flow system (another integrated fan) sucks the mosquitoes into a net where they die from dehydration. The MM trap was chosen over 
other trapping alternatives for the following reasons: 1) this trap captures blood seeking mosquitoes (mosquitoes that could potentially transmit VBD when biting their hosts) attracted by $\mathrm{CO}_{2}$; 2) MM traps are the only available trap type that allows the autonomy needed for this sampling scheme; 3) In comparison with other trap types where $\mathrm{CO}_{2}$ is provided by, for example, dry-ice or $\mathrm{CO}_{2}$ tanks, $\mathrm{CO}_{2}$ autonomy is provided by an easy to purchase and replace propane tank; 4) This trap type was also chosen and used for the MODIRISK national inventory of mosquitoes in Belgium (Versteirt et al. 2013), enabling the possibility of comparing results.

\section{Sampling frequency and field operations:}

Mosquitoes were sampled from mid-April until the second half of October (mosquito season) during the years 2010, 2011, 2012 and 2013. To increase the efficiency of the sampling strategy, and to evenly distribute the sampling sites in the study area and during the mosquito season, the entire country was sampled twice every year. In this way, half of the random points created for the mosquito season were sampled from the middle of April until the middle of July (run 1), and the other half from the middle July until the middle of October (run 2) (Figure 1). Sampling sites were grouped by region (total six working regions), and linked to a field inspector. Each site was sampled once for seven days with an MM trap. After this, the trap was placed in the next sampling site belonging to the same region/inspector. The six inspectors had the possibility of visiting two sites (two traps) in one week, visit only one site, or skip one week sampling. An average of seven traps operated simultaneously during the campaign. Despite the fact that each sampling site was only visited once, the total amount of sampling sites sampled in the vicinity gives an overview of mosquito species present in a specific area. Instructions were given to place the trap as close as possible to the provided XY random coordinates. If it was not possible (e.g. in some urban areas with the presence of children, or the presence of temporary water bodies on location), the trap could be moved to a safe place within $100 \mathrm{~m}$ of the original XY coordinates. In that case, new coordinates were recorded. Field information, sampling information (inspector name, site number, trap start date, trap end date), $X Y$ coordinates recorded with a Global Positioning System (GPS), and one unique sampling number barcode were recorded in a fieldwork data form. Trapping nets from the MM trap with collected mosquitoes and fieldwork dataform were sent together inside a sealed plastic bag to the laboratory for morphological identification. All data from each sampling location were input into VecBase (a tailor-made application built for CMV using Oracle Application Express (Oracle Corporation 2009)), with 
details on $\mathrm{XY}$ coordinates, field inspector, number of days in the field, species captured, quantity, sex (male, female), etc. VecBase provided options to manage the stored data and to make specific queries (ECDC 2012).

\section{Morphological identification}

The contents of the traps were collected each week and sent to the CMV laboratory. In the laboratory, mosquitoes were morphologically identified by specialists on European mosquito species using, among others, the Culicidae key specifically designed for rapid field-identification of Dutch adult Culicidae (modified key after (Snow 1990, Schaffner et al. 2001, Verdonschot 2002, Becker et al. 2010)). Exotic species were identified using the electronic identification key of (Schaffner et al. 2001). Afterwards, sampling results of morphological identification were introduced in VecBase. To allow specimen validation, and to provide specimens for subsequent molecular identification, samples from this study were stored dry inside a plastic Petri dish with absorbent paper in a $-20^{\circ} \mathrm{C}$ freezer.

\section{Data analysis}

Diversity (as defined by (Whittaker 1972)) of the defined three habitats was calculated using richness and evenness indices. Sim pson's Index of diversity $(1-\mathrm{D}=1-\Sigma[n \mathrm{i} \times(n \mathrm{i}-1) / N \times(N$ $-1)]$ ), where $n \mathrm{i}$ is the number of the $\mathrm{i}$-th species and $N$ is the number of individuals in the studied defined habitat), is a measurement of the probability that two randomly selected individuals in an area belong to a different species. The value of this index ranges between 0 and 1 and the greater the value is, the greater the sample diversity. Shannon-Wiener index $\left(\mathrm{H}^{\prime}=-\Sigma \mathrm{pi} \times \ln \mathrm{pi}\right)$, where pi is the proportion of the $\mathrm{i}$-th species in the studied habitat, was used as a measure of community heterogeneity (Krebs 1989), and Shannon evenness $\left(E^{\prime \prime}=S / \ln \left(H^{\prime}\right)\right.$, where $S$ is the total number of species), was used as a measure of biodiversity which quantifies how equal the community is numerically. The value of this index also ranges between 0 and 1 , with 1 being complete evenness.

The Kruskal-Wallis one-way analysis of variance was used to test whether there was a difference in sampling results between seasons and years. To obtain robust statistics we considered the 99.5 percentile of the data (Hodge and Austin 2004). 


\section{Results}

During the survey, 792 random locations were selected for mosquito sampling in the three described basic habitats types (urban, rural-agricultural and natural), 98\% $(n=778)$ of these being effectively sampled and in $91 \%(n=707)$ of the locations mosquitoes were collected (see Table 1). Details about the number of sites sampled, biodiversity and abundance in each category are summarized in Table 2.

Table 1. Total number of sampled locations from 2010 until 2013

\begin{tabular}{lrrrrr}
\hline \hline Sampled locations & 2010 & 2011 & 2012 & 2013 & Total \\
\hline $\begin{array}{l}\text { No. sampled locations } \\
\begin{array}{l}\text { No. sampled locations without } \\
\text { mosquitoes }\end{array}\end{array}$ & 254 & 224 & 151 & 149 & 778 \\
\hline
\end{tabular}

Table 2. The total number of sampled locations, species collected, specimens collected and mean number of specimens per trap in each habitat type.

\begin{tabular}{lrrr}
\hline \hline Taxonomic diversity & Urban & Agricultural & Natural \\
\hline No. sampled locations & 309 & 314 & 155 \\
No. species collected & 23 & 23 & 23 \\
No. of specimen collected & 8,927 & 9,924 & 19,857 \\
Mean no. specimens per trap & 28.89 & 31.61 & 128.11 \\
Simpson Index & 0.755 & 0.756 & 0.874 \\
Shannon-Wiener diversity & 1.801 & 1.808 & 2.234 \\
Shannon-Wiener evenness & 0.574 & 0.576 & 0.712 \\
\hline
\end{tabular}

In total, 38,708 mosquitoes were captured and 27 mosquito species (or species complexes) from five genera were found (see Table 3). Due to the extremely similar morphology of the adult females of Cx. pipiens (Linnaeus, 1758) and Cx. torrentium (Martini, 1925), Ae. annulipes (Meigen, 1830) and Ae. cantans (Meigen, 1818), and the An. maculipennis complex (An. atroparvus (van Thiel, 1927), An. maculipennis s.s. (Meigen, 1818), An. melanoon (Hackett, 
Table 3. Total number of species collected, total number of sites were the species were caught, total number of specimens per species and its habitat type in the Netherlands.

\begin{tabular}{|c|c|c|c|}
\hline Species & $\begin{array}{c}\text { No. sites species found } \\
\text { (\% all sites) }\end{array}$ & $\begin{array}{l}\text { Total no. of specimens } \\
\text { collected ( } \% \text { of total) }\end{array}$ & Habitat type \\
\hline Ae. annulipes/cantans & $50(6.43)$ & $1,546(3.99)$ & All \\
\hline Ae. caspius & $2(0.26)$ & $5(0.01)$ & Natural \\
\hline Ae. cinereus & $106(13.62)$ & $3,222(8.32)$ & All \\
\hline Ae. communis & $23(2.96)$ & $168(0.43)$ & All \\
\hline Ae. detritus & $5(0.64)$ & $7(0.02)$ & Natural, Agricultural \\
\hline Ae. dorsalis & $6(0.77)$ & $15(0.04)$ & All \\
\hline Ae. flavescens & $9(1.16)$ & $2,454(6.34)$ & All \\
\hline Ae. geniculatus & $51(6.56)$ & $416(1.07)$ & All \\
\hline Ae.j.japonicus & $2(0.26)$ & $2(0.01)$ & Natural, Urban \\
\hline Ae. leucomelas & $5(0.64)$ & $23(0.06)$ & All \\
\hline Ae. nigrinus & $2(0.26)$ & $40(0.10)$ & Urban, Agricultural \\
\hline Ae. punctor & $73(9.38)$ & $1,168(3.02)$ & All \\
\hline Ae. riparius & $1(0.13)$ & $1(0.002)$ & Agricultural \\
\hline Ae. rusticus & $4(0.51)$ & $34(0.09)$ & All \\
\hline Ae. sticticus & $14(1.80)$ & $91(0.24)$ & All \\
\hline Ae. vexans & $17(2.19)$ & $11,096(28.67)$ & All \\
\hline An. claviger & $130(16.71)$ & $549(1.42)$ & All \\
\hline An. maculipennis s.l. & $144(18.51)$ & $681(1.76)$ & All \\
\hline An. plumbeus & $100(12.85)$ & $2,267(5.86)$ & All \\
\hline Cq. richiardii & $155(19.92)$ & $2,547(6.58)$ & All \\
\hline Cs. annulata & $451(57.97)$ & $5,007(12.94)$ & All \\
\hline Cs. fumipennis & $6(0.77)$ & $47(0.12)$ & All \\
\hline Cs. morsitans & $3(0.39)$ & $7(0.02)$ & Natural, Urban \\
\hline Cs. subochrea & $1(0.13)$ & $1(0.002)$ & Natural \\
\hline Cx. modestus & $2(0.26)$ & $2(0.01)$ & Agricultural \\
\hline Cx. pipiens/torrentium & $455(58.48)$ & $7,025(18.15)$ & All \\
\hline Cx. territans & $3(0.39)$ & $3(0.01)$ & All \\
\hline
\end{tabular}




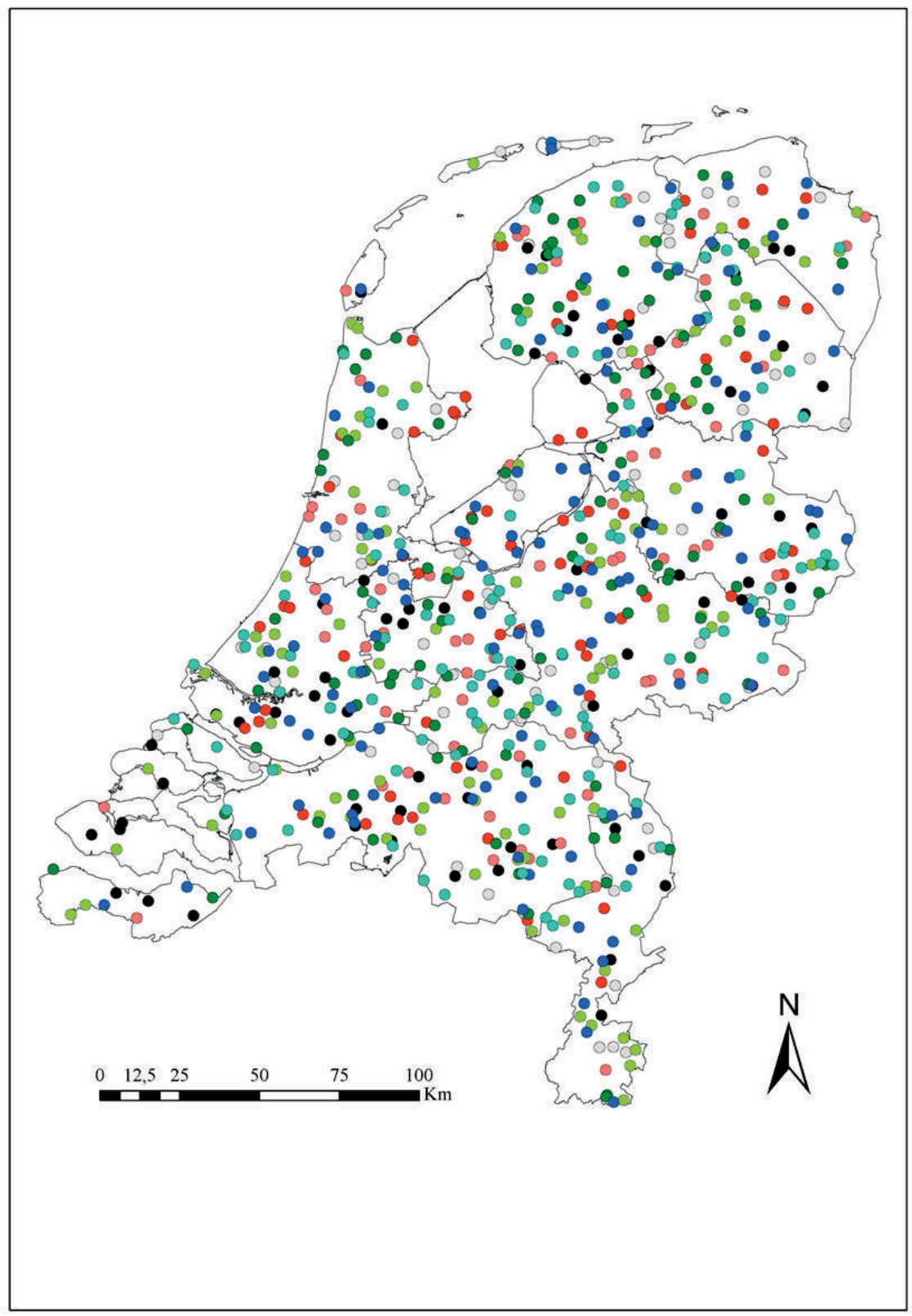

Fig 1. The Netherlands, showing location of mosquito sampling sites during NVS-mosquitoes (2010-2013). Dark blue dots: sampling sites 2010, run 1; light blue dots: sampling sites 2010, run 2; dark green dots: sampling sites 2011, run 1; light green dots: sampling sites 2011, run 2; red dots: sampling sites 2012, run 1; pink dots: sampling sites 2012, run 2; black dots: sampling sites 2013, run 1; grey dots: sampling sites 2013, run 2. 
1934) and An. messeae (Falleroni 1926)), specimens in these groups were not identified to species level (e.g. Cx. pipiens/torrentium, Ae, annulipes/cantans, and An. maculipennis s.1.). The most species-rich genus in the Netherlands is Aedes, whereas Coquillettidia was only represented by one species. The most widespread species found in the sampling locations were $C x$. pipiens/torrentium and Culiseta annulata (Schrank, 1776) being present in 58.48\% and 57.97\% of all samples collected (Table 3). Other frequent species, present in at least 100 locations were Coquilletidia richiardii (Ficalbi, 1889) (19.92\%), An. maculipennis s.1. (18.51\%), An. claviger (Meigen, 1804) (16.71\%), Ae. cinereus (Meigen, 1818) (13.62\%) and An. plumbeus (Stephens, 1828 ) (12.85\%). The five most abundant species were Ae. vexans (Meigen, 1830) (28.67\%), Cx. pipiens/torrentium (18.15\%), Cs. annulata (12.94\%), Ae. cinereus $(8.32 \%)$ and Cq. richiardii (6.58\%). Only four species considered to be indigenous were not captured during this survey: An. algeriensis (Theobald, 1903), Cs. ochroptera (Peus, 1935), Cs. alaskaensis (Ludlow, 1906) and Ae. excrucians (Walker, 1856). One exotic species, Aedes j. japonicus, was found during the survey in two consecutive years (2012-2013) in two trap locations in the same municipality (Lelystad, Flevoland province).

About $28.67 \%$ of the individuals collected in the survey belong to one single species Ae. vexans, but this is heavily influenced by one single sampling during one week in the survey in 2011 where thousands of Ae. vexans individuals were collected. Culex pipiens/torrentium made up $18.1 \%$ of the mosquitoes collected, and is considered the most abundant species in the study with 7,025 individuals collected in 455 sampled locations.

Mosquitoes were captured in a wide variety of land use classes of the National Land Use Map File of The Netherlands (LGN5), including vegetated dunes, salt marshes, buildings in urban areas, forest in swamp areas, pastures, etc. From the 39 land use classes, no mosquito sampling sites were randomly assigned to seven land use classes: bare soil in built-up area, heathlands in dune areas, shifting sands, heathlands, heathlands with minor grass influence, forest in raised bogs, and swampy pastures in peat areas. Nineteen species of the 27 species captured in this study were present in all three classified habitats considered (Figure 2). This group includes the most widespread species Cx. pipiens/torrentium, Cs. annulata, An. maculipennis s.1., Cq. richiardii and An. claviger. Species captured in one habitat type, or in a combination of two habitat types are Ae. detritus (Haliday, 1833), Cs. morsitans (Theobald, 1901), Cx. modestus (Ficalbi, 1890), Ae. caspius (Pallas, 1771), Ae. j. japonicus, Ae. nigrinus (Eckstein, 1918), Ae. 
riparius (Dyar \& Knab, 1907) and Cs. subochrea (Edwards, 1921). This group of species was captured in the study in five or fewer sampling sites (see Table 3). The total number of species captured in each habitat was the same for all three habitats $(n=23)$, but calculated species diversity (H'+Sim pson) and evenness (E') indicate higher richness and evenness in natural sampled sites in comparing with agricultural and urban sites (Table 2). Urban and agricultural sites showed almost identical species diversity and evenness (Table 2).

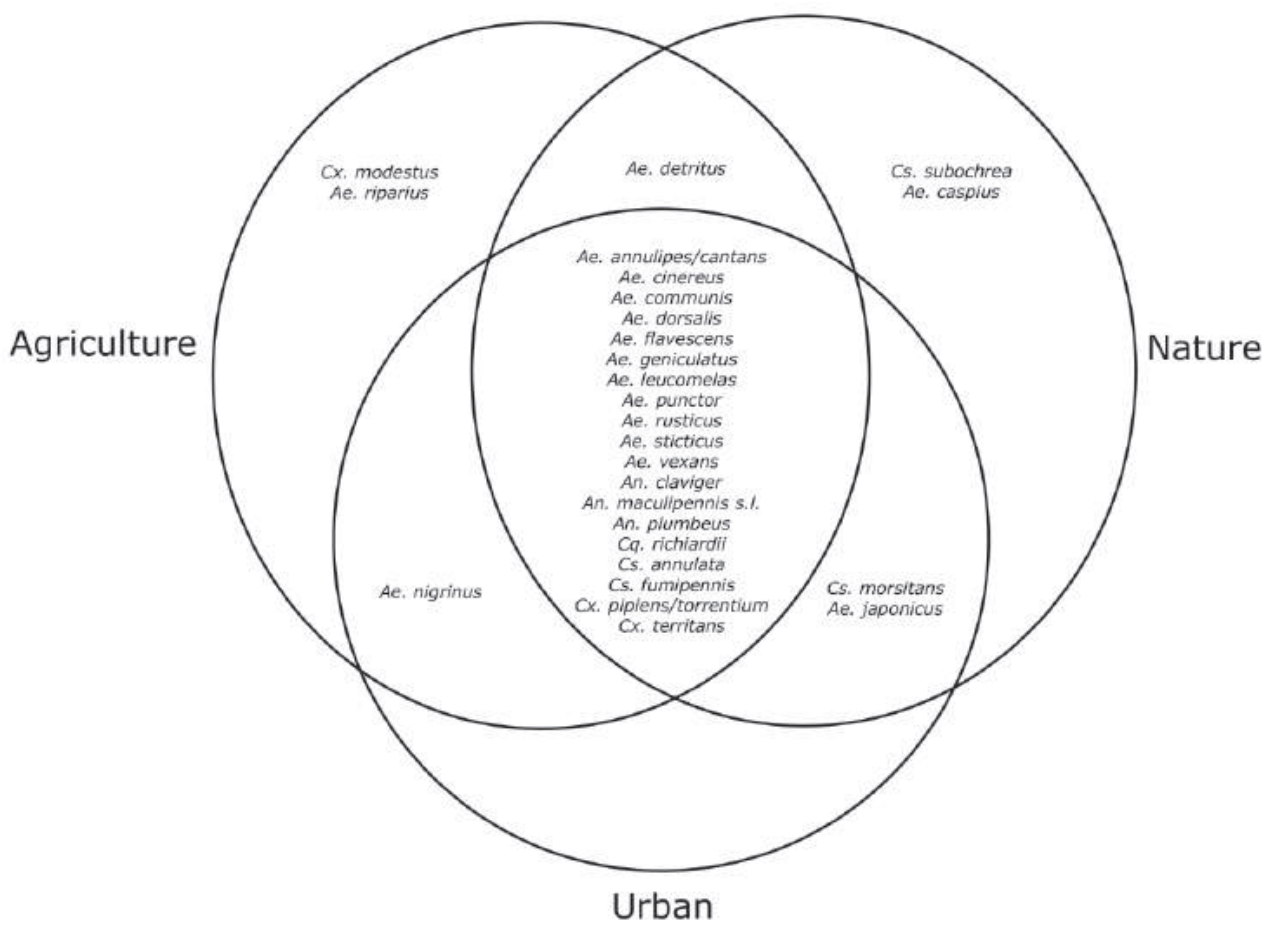

Figure 2. Habitat classification of mosquitoes species collected during the NVS-mosquitoes, year 2010-2013.

Species composition was not identical every year, and significant differences in abundance were found across the years and seasons. There was no significant difference between years 20102012, while in 2013 there was a significant decrease in the number of mosquitoes (KruskalWallis test, $\mathrm{df}=1, \mathrm{P}<0.05)$. We also observed that there was significant increase of captures from the middle of July onwards (run2) when compared with the captures between April and July (run1) (Kruskal-Wallis test, $\mathrm{df}=1, \mathrm{P}<0.05$ ). 
The survey has provided data on adult periodicity for the species captured (Figure 3), and maps depicting the geographical occurrence of these species in the country (Figure 4). Diseases putatively transmitted by these mosquito species are given in Table 4 .

\section{Discussion}

In a previous longitudinal study performed in the Netherlands in 2009-2010 (Versteirt et al. 2009), 22 indigenous species were reported. In comparison, this study detected the presence of 26 species considered to be indigenous in the Netherlands (Verdonschot 2002), and one invasive mosquito species. Given that species groups (e.g. Cx. pipiens/torrentium, Ae. annulipes/cantans, and An. maculipennis s.1.: An. atroparvus, An. maculipennis s.s., An. melanoon and An. messae) were considered together for morphological reasons, it is possible that as many as 32 species (31 indigenous and one invasive mosquito species) may have been detected in this study. The Dutch mosquito fauna listed in the checklist for dipterans in the Netherlands (Verdonschot 2002) includes a total of 35 indigenous mosquito species. Therefore the four species not detected in this study (Verdonschot 2002) includes An. algeriensis, Cs. ochroptera, Cs. alaskaensis, and Ae. excrucians. Aedes excrucians was found during a survey performed in 2005 in the Netherlands only in one out of 12 locations (Oostvaardersplassen wetland, W. Takken, personal communication) using the same type of trap. Anopheles algeriensis was also found in the same area in 2009 during a specific study of arbovirus circulation in this wetland (Scholte et al. 2011). No recent geo-referenced records of Cs. alaskaensis and Cs. ochroptera are known to us. The absence of these four species can be attributed to different reasons: 1) rareness of these four species within the country; 2) the trap type device used could be inappropriate for capturing this species (e.g. An. algeriensis was captured using CDC Miniature Light traps), and 3) even if the species could occur in a sampled site, the species could be in a non-detectable stage (egg, pupa, or larvae). In addition to the indigenous species captured during the survey, the invasive mosquito species Ae.j. japonicus was captured in two different locations in the municipality of Lelystad in two consecutive years. Females of this invasive species could be detected using this strategy, giving a reason to use this strategy, not only to better understand the indigenous mosquito fauna, but also as a passive surveillance technique for the detection of hitherto unknown populations of established invasive mosquito species. 
Table 4. Overview diseases putatively transmitted by mosquito species collected in during the NVSmosquitoes survey 2010 - 2013 (adapted from vector-disease relationships mentioned in Becker et al.,2010, Schaffner et al., 2001 and Versteirt et al., 2011.). (*) No parasitic disease transmission has been assigned to this species.

\begin{tabular}{|c|c|c|}
\hline Mosquito species & Naturally infected & Lab infected only \\
\hline Aedes annulipes & Myxomatosis, Tahyna, West Nile & \\
\hline Aedes cantans & Myxomatosis, Tahyna, West Nile & \\
\hline Aedes caspius & $\begin{array}{l}\text { Myxomatosis, Tahyna, Tularemia, } \\
\text { West Nile }\end{array}$ & $\begin{array}{l}\text { Dirofilaria immitis, Dirofilaria } \\
\text { repens }\end{array}$ \\
\hline Aedes cinereus & Sindbis , Tahyna , Tularemia & \\
\hline Aedes communis & Batai , Inkoo, Tularemia & Tahyna \\
\hline Aedes detritus & Dirofilaria repens, Myxomatosis & \\
\hline Aedes dorsalis & $\begin{array}{l}\text { California enceohalitis, St. Louis } \\
\text { encephalitis, Tularemia, Western equine } \\
\text { encephalitis }\end{array}$ & Japanese Encephalitis \\
\hline Aedes flavescens & & Tahyna, Tularemia \\
\hline Aedes geniculatus & Tularemia & West Nile, Yellow fever \\
\hline Aedes j. japonicus & Japanese encephalitis, West Nile & $\begin{array}{l}\text { La Crosse, Chikungunya, Dengue, } \\
\text { Eastern equine encephalitis, St. } \\
\text { Louis encephalitis }\end{array}$ \\
\hline \multicolumn{3}{|l|}{ Aedes leucomelas (*) } \\
\hline $\begin{array}{l}\text { Aedes punctor } \\
\text { Aedes riparius }(*) \\
\text { Aedes rusticus }(*)\end{array}$ & Inkoo, Tularemia & Tahyna, West Nile \\
\hline Aedes sticticus & Inkoo, Tahyna & \\
\hline Aedes vexans & $\begin{array}{l}\text { California encephalitis, Dirofilaria } \\
\text { immitis, Eastern equine encephalits, } \\
\text { Lednice, Rift valley fever, Tahyna, } \\
\text { Tularemia, Western Equine } \\
\text { Encephalitis, West Nile }\end{array}$ & \\
\hline Anopheles claviger & $\begin{array}{l}\text { Anaplasmosis, Batai, Dirofilaria } \\
\text { immitis, Malaria, Setaria } \\
\text { labiatopapillosa, Tahyna, Tularemia }\end{array}$ & Myxomatosis \\
\hline Anopheles maculipennis s.l. & $\begin{array}{l}\text { Batai, Dirofilaria immitis, Malaria, } \\
\text { Tahyna, Myxomatosis, Tularemia, West } \\
\text { Nile }\end{array}$ & \\
\hline Anopheles plumbeus & Malaria & Dirofilaria immitis, West Nile \\
\hline Coquillettidia richiardii & $\begin{array}{l}\text { Batai, Omsk haemorrhagic fever, } \\
\text { Tahyna, West Nile }\end{array}$ & \\
\hline Culex modestus & $\begin{array}{l}\text { Dirofilaria immitis, Lednice, Sindbis, } \\
\text { Tahyna, Tularemia, West Nile } \\
\text { Myxomatosis }\end{array}$ & \\
\hline Culex pipiens & $\begin{array}{l}\text { Avian malaria, Batai, Sindbis, Usutu, } \\
\text { West Nile }\end{array}$ & Dirofilaria immitis, Tahyna \\
\hline Culex territans & Batrachian filariasis & \\
\hline Culex torrentium & Sindbis & \\
\hline $\begin{array}{l}\text { Culiseta annulata } \\
\text { Culiseta fumipennis }(*)\end{array}$ & Avian malaria, Tahyna & Myxomatosis \\
\hline Culiseta morsitans & Sindbis & \\
\hline Culiseta ochroptera & Sindbis & \\
\hline
\end{tabular}


Seventy percent of the species $(n=19 / 27)$ were captured in all three selected and reclassified habitat types (Figure 2). This result could be explained by looking at the landscape structure of the Netherlands, characterized by high fragmentation, and where urban and rural habitats are adjacent and connected to natural habitats, even in small areas. For instance, in a $1 \mathrm{Km}^{2}$ pixel it is not difficult to find all three habitat types. Considering the wide flight range of several bloodseeking mosquito species, and the fragmentation of habitats, it was possible for a salt marsh mosquito species such as Ae. dorsalis (Meigen, 1830), only expected to be found in natural areas, to also be captured in neighboring urban and agricultural areas, or for a forest mosquito such as Ae. rusticus (Rossi, 1790) to be captured in a park in the cosmopolitan city of Amsterdam.

The phenology of the species varies (Figure 3), showing that some species become active early in the spring and are active during the whole season (e.g. Cx. pipiens/torrentium), while others had a distinctly shorter season and appear later in the spring and end earlier in the season (e.g. Ae. vexans). Data on the phenology of the mosquito species provided by the NVS survey (Figure 3) can usefully depict the activity period of the adult mosquitoes in the Netherlands only for species captured on a representative number of locations (e.g. Cs. annulata). However, for mosquito species in the Netherlands that were not frequently captured (e.g. Cx. modestus), these phenology data can only give an estimate of adult activity. Information on the phenology and abundance of a mosquito species is crucial in understanding its role as a disease vector. For example, abundance of Cx. pipiens increases drastically from mid-July (Kruskal-Wallis test, $\mathrm{P}<0.01, \mathrm{df}=1$ ), being in the second sampling period (run2) when it is expected that West Nile virus would more likely be circulating.

Due to the difficulty of morphologically identifying $C x$. pipiens and $C x$. torrentium adult mosquitoes, specimens of this group were identified as $C x$. pipiens/torrentium. Recent outbreaks of WNV in Southern Europe (Balenghien et al. 2008) have implicated members of the $C x$. pipiens complex mosquitoes (Cx. pipiens pipiens, $C x$. pipiens molestus or hybrids), and recent outbreaks of Sindbis virus have implicated the species $C x$. torrentium (Hesson et al. 2011). For these reasons, molecular investigations based on a real-time PCR version of Rudolf et al. (2013) are in progress with the goal of analyzing the majority of the Cx. pipiens/torrentium samples included in this study in order to provide better information on the geographical distribution of the $C x$. pipiens complex and $C x$. torrentium in the Netherlands. 


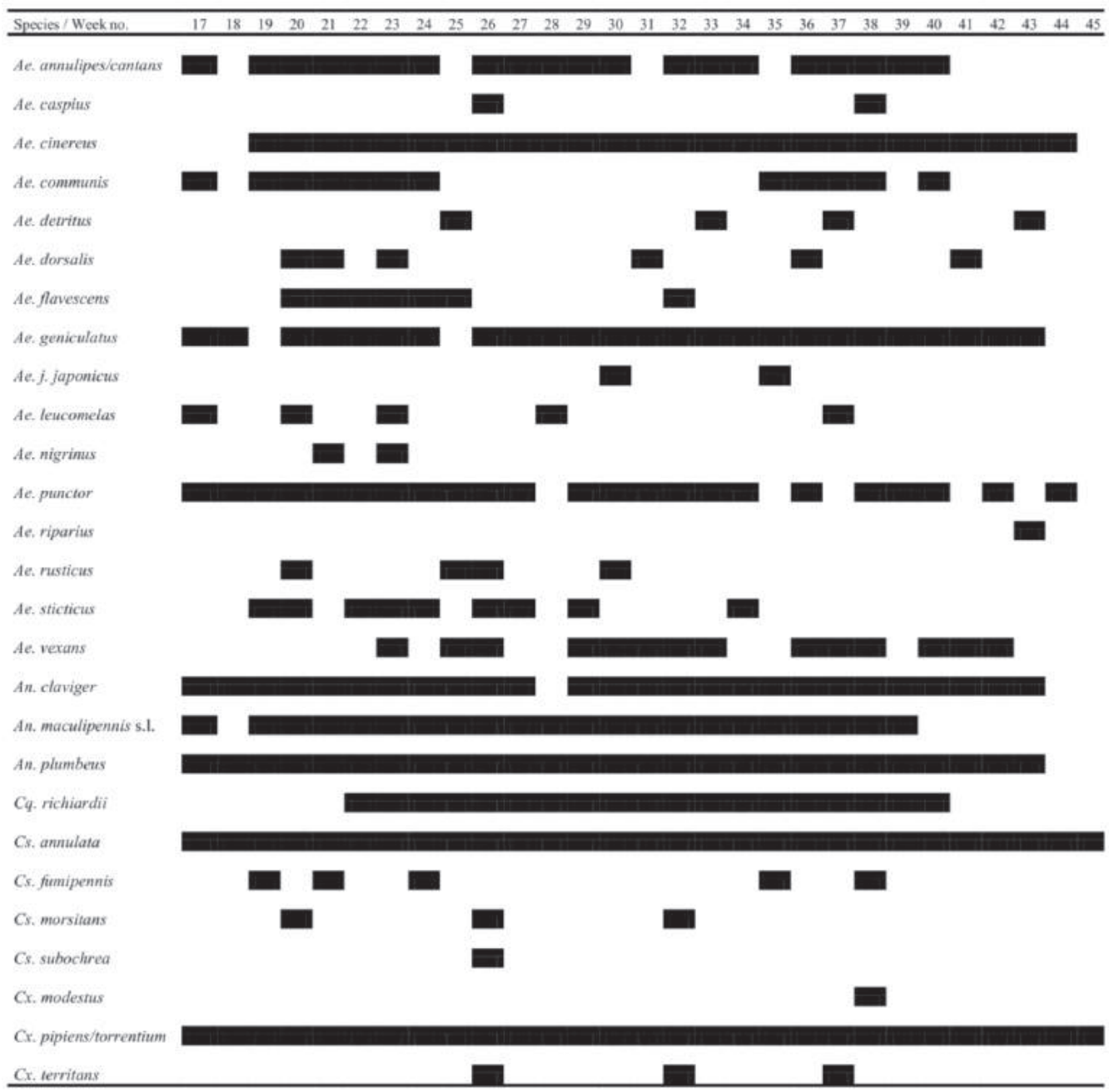

Figure 3. Seasonal occurrence of mosquitoes species collected during the NVS-mosquitoes, year 2010-2013.

The most abundant species (species with most individuals collected during the study) for the Netherlands is the potential vector of RVFV, Ae. vexans (Fischer et al. 2013). Among all mosquitoes captured, $28.67 \%$ of the captures belong to this aggressive floodplain mosquito, with most (more than 10,000 individuals) captured in a single location. This result shows the ability of this species to appear in high numbers after an area containing Ae. vexans eggs is inundated. Similar results were encountered for the mosquito species Ae. flavescens (Müller, 1764). This species is not reported as occurring in great numbers by (Becker et al. 2010), but is reported as a 

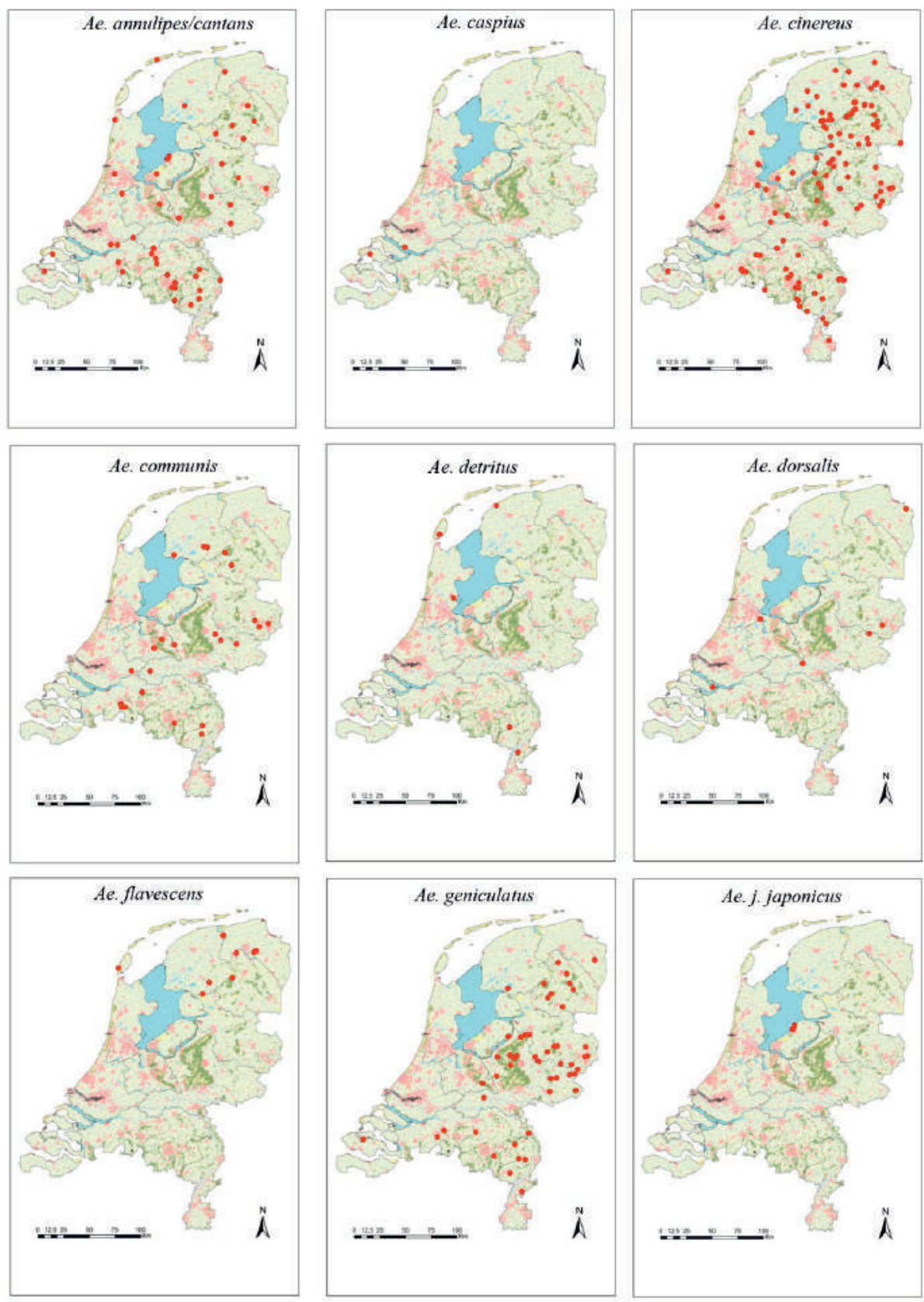

Figure 4. The Dutch geographical distribution of: Aedes annulipes/cantans, Ae. caspius, Ae. cinereus, Ae. communis, Ae. detritus, Ae. dorsalis, Ae. flavescens, Ae. geniculatus and Ae. j. japonicus. Red dot: species records during study 2010-2013. 
species that can cause an important nuisance in Northern Europe (Schaffner et al. 2001). In a natural floodplain near a lake site in the northern province of Friesland, 2,400 specimens were collected during one week, indicating the possibility of temporal high abundance of this species in floodplain areas, depending on the availability of floodwater pools.

From all the sampling sites, $18 \%$ contained individuals of the An. maculipennis complex and were found in all three habitat types. In the Netherlands, four members of the complex are listed as present (Verdonschot 2002): An. atroparvus, An. messae, An. maculipennis s.s. and An. melanoon, the last species not referenced as present in the Netherlands by Takken et al. (2007), and An. maculipennis s.s. considered as present but not common. In this study, no morphological or molecular identification of the captured An. maculipennis s.l. was performed. Investigations by Takken et al. (2002) and recent investigations on overwintering mosquitoes (Scholte et al. 2013) confirm An. messae as the dominant and abundant species of the complex in the Netherlands and An. atroparvus (the only incriminated as malaria (Plasmodium vivax and Plasmodium malariae) vector in the Netherlands (Takken et al. 2002)) as more confined to coastal areas (presence of brackish water) and less abundant. An. maculipennis s.s. was not found during the Scholte et al. (2013) study.

An. claviger and An. plumbeus are the fifth and seventh most frequent mosquito species in the country. The wetland mosquito An. claviger is only considered as a malaria vector in Southern Europe and it has never been involved in malaria in temperate climate zones (Schaffner et al. 2003), but the tree-hole mosquito An. plumbeus has been involved in cases of indigenous malaria transmission in Germany (Kruger et al. 2001) and has proven to be a moderate to highly receptive of $P$. falciparum, the most deadly form of malaria (Schaffner et al. 2012), and laboratory vector of WNV (Schaffner et al. 2001). This species was found in all three habitat types, including areas where forest was not present. Nowadays, it is usual to find high populations of this active day biting species in recently abandoned farms with underlying water contaminated with organic waste in rural areas of the country. This situation causes nuisance to the humans living in the surroundings, and eventually could be responsible for autochthonous malaria cases, created by the chance of putting in contact infected P. falciparum humans (travellers/temporary workers) with a high density of this malaria vector. 

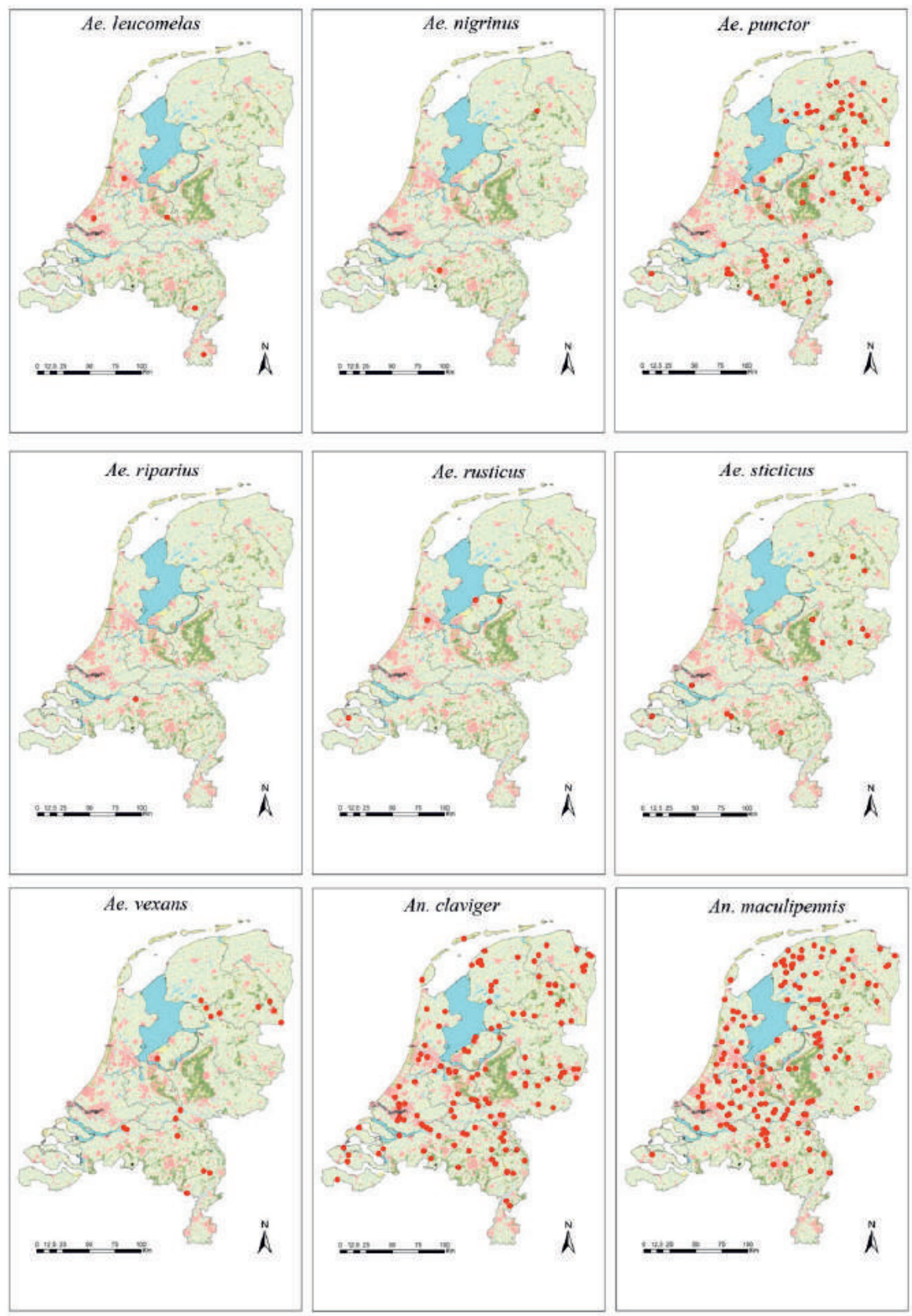

Figure 5. The Dutch geographical distribution of: Aedes leucomelas, Ae. nigrinus, Ae. punctor, Ae. riparius, Ae. rusticus, Ae. sticticus, Ae. vexans, Anopheles claviger and An. maculipennis s.l. Red dot: species records during study 2010-2013. 
The NVS study was based on a cross-sectional and random (statistical) approach. Random sampling points were chosen to avoid biased choice of sampling sites. Using this methodology the entire country could be sampled over different years and periods, providing data on the mosquito species collected all over the country. Deficiencies in the sampling scheme were assumed, such as the use of only one trapping device, only one week of trapping on each site, and the necessity to lead with areas or specific habitats that may have been insufficiently sampled or sampled during the wrong flying period of adult mosquitoes. However, comparing the number of found and expected species, the detection of one exotic mosquito species (Ae. $j$. japonicus), and the provided mosquito diversity results using this methodology, it can be concluded that this survey approach was efficient. Similar methodology and the same trap type was also used for the MODIRISK national inventory of mosquitoes in Belgium (Versteirt et al. 2013) giving satisfactory results. In both studies, one exotic mosquito species was collected from random generated sampling sites. Ae. j. japonicus in the NVS study and Ae. koreicus in MODIRISK. In addition, in MODIRISK, Ae. j. japonicus was also collected but in a manually selected importation site. In contrast to the MODIRISK project, no molecular tests were planned for the present study, but because the sample collections are currently stored, further molecular studies with the aim of molecularly identify between $C x$. pipiens/torrentium and for the An. maculipennis s.l. species can be perform ed. MODIRISK's sam pling strategy included selected sites in import risk areas (IRA) nature (zoos, safari parks, protected areas involving the presence of large numbers of migratory birds where importation of pathogens is possible) and IRAindustry (importation risk areas for exotic mosquitoes such as used tire importation/storage companies, bamboo importers, harbours and airports) areas. In NVS, IRA nature areas are also included in the sampling area but locations were not selected, but sampled in the same way as the rest of the sites (random sampling locations). IRA-industry sites included in MODIRISK were not previously selected for sampling in the NVS survey. From the 778 sampled sites, several could be randomly assigned to the vicinity of used tire import/storage companies or lucky bamboo import companies, but no prior selection of the import risk location was done because this is not the purpose of this survey. For the purpose of detecting introduction of exotic mosquitoes, an especially designed Exotic Mosquito Survey has been carried out by the CMV from 2010 onwards providing data on the detection of exotic/invasive mosquitoes (Scholte et al. 2010a, Scholte et al. 2010b, Scholte et al. 2012, Scholte et al. 2014). 

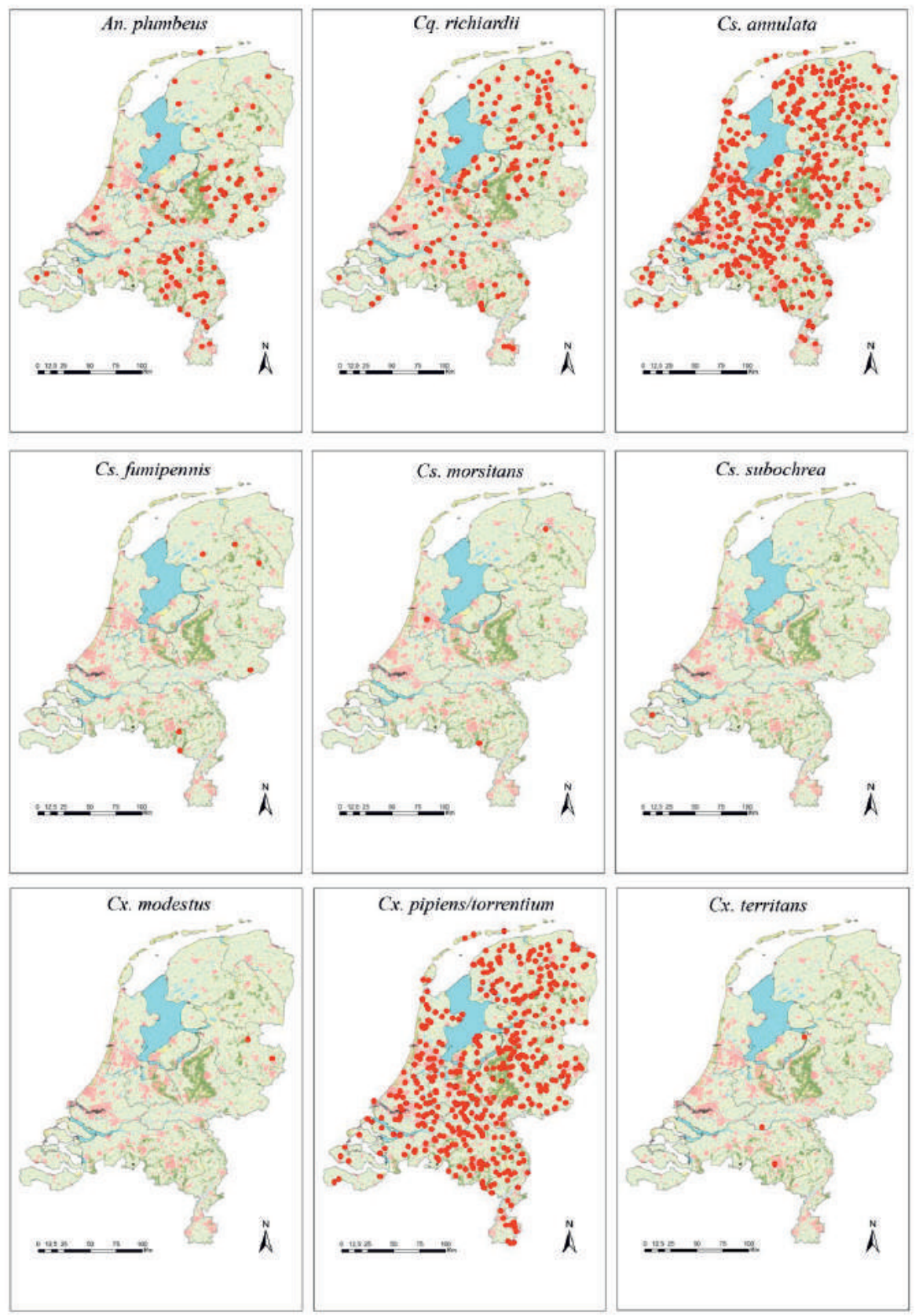

Figure 6. The Dutch geographical distribution of: Anopheles plumbeus, Coquillettidia richiardii, Culiseta annulata, Cs. fumipennis, Cs. morsitans, Cs. subochrea, Culex modestus, Cx. pipiens/torrentium and Cx. territans. Red dot: species records during study 2010-2013. 
The most important and unique value of this study is the resulting nationwide geographical distribution of the mosquito fauna. Prior mosquito studies in the country focused only on species composition and phenology, and not on geographical distribution of species. Here we add a unique value to the mosquito fauna composition. Maps of presence/absence of mosquitoes at randomly selected locations, created unbiased data that can be used for develop mosquito species distribution models. These maps are a solid base for risk analysis of vector borne diseases transmitted by mosquitoes, useful in case of the introduction of a VBD transmitted by indigenous vector competent mosquito species. Mapping the distribution of the mosquito species in the Netherlands using this methodology is strongly recommended as a routine surveillance given the potential risk of mosquito-borne diseases in Europe.

\section{Acknowledgements}

During the preparation of this manuscript, our respected and beloved colleague Ernst-Jan Scholte passed away due to cancer. Even after the diagnosis and treatment, Ernst-Jan was enthusiastic about this work and he spent a lot of time discussing the first drafts. On behalf of the authors of this publication we would like to thank Ernst-Jan Scholte for the inspiration, his support and great contribution. We would also like to thank Dr. Jolyon Medlock and Ms. Tessa Bremen for reviewing the English in the manuscript, Dr. Alberto Llera Arenas for helping with the statistical analysis of the data, the inspectors of the Netherlands Food and Consumer Product Safety Authority (NVWA) for the excellent field work, and the private home owners and nature organizations for giving us permission for sampling on their properties. 



\section{Chapter 3}

\section{Modelling the spatial distribution of the nuisance mosquito species Anopheles plumbeus (Diptera: Culicidae) in the Netherlands}

Adolfo Ibáñez-Justicia ${ }^{1}$ and Daniela Cianci ${ }^{2}$

1. Centre for Monitoring of Vectors (CMV), Netherlands Food and Consumer Product Safety Authority (NVWA), Wageningen, The Netherlands

2. Faculty of Veterinary Medicine, Utrecht University, Utrecht, The Netherlands

Published in Parasites \& Vectors (2015) 8(1):258 doi:10.1186/s13071-015-0865-7 


\section{Abstract}

Landscape modifications, urbanization or changes of use of rural-agricultural areas can create more favourable conditions for certain mosquito species and therefore indirectly cause nuisance problems for humans. This could potentially result in mosquito-borne disease outbreaks when the nuisance is caused by mosquito species that can transmit pathogens. Anopheles plumbeus is a nuisance mosquito species and a potential malaria vector. It is one of the most frequently observed species in the Netherlands. Information on the distribution of this species is essential for risk assessments. The purpose of the study was to investigate the potential spatial distribution of An. plumbeus in the Netherlands. Random forest models were used to link the occurrence and the abundance of An. plumbeus with environmental features and to produce distribution maps in the Netherlands. Mosquito data were collected using a cross-sectional study design in the Netherlands, from April to October 2010-2013. The environmental data were obtained from satellite imagery and weather stations. Statistical measures (accuracy for the occurrence model and mean squared error for the abundance model) were used to evaluate the models performance. The models were externally validated. The maps show that forested areas (centre of the Netherlands) and the east of the country were predicted as suitable for An. plumbeus. In particular high suitability and high abundance was predicted in the south-eastern provinces Limburg and North Brabant. Elevation, precipitation, day and night temperature and vegetation indices were important predictors for calculating the probability of occurrence for An. plumbeus. The probability of occurrence, vegetation indices and precipitation were important for predicting its abundance. The AUC value was 0.73 and the error in the validation was 0.29 ; the mean squared error value was 0.12. The areas identified by the model as suitable and with high abundance of An. plumbeus, are consistent with the areas from which nuisance was reported. Our results can be helpful in the assessment of vector-borne disease risk.

Keywords Species distribution modelling, Anopheles plumbeus, Mosquito nuisance, Vectorborne diseases, Random forest 


\section{Background}

Mosquitoes (Diptera: Culicidae) are known to be vectors of a large number of pathogens around the globe and are considered as prime candidates for transmitting (re-)emerging vector-borne diseases (VBDs) in Europe (Versteirt et al. 2009). The increased mobility of humans, that has also increased the mobility of livestock and pathogens, as well as environmental modifications and climate changes can contribute to the (re-)emergence of vector-borne diseases (Randolph and Rogers 2010). Furthermore, mosquito bites can cause a considerable nuisance for humans and mammals. Severe nuisance can have negative economic consequences (e.g., in tourism, work productivity outdoors, meat and dairy production) (Connelly and Carlson 2009). These nuisance situations can eventually lead to autochthonous VBD cases, when in non-endemic areas infectious reservoirs, either humans (travellers, temporary workers) or animals (livestock, migrating animals) come in contact with high density of mosquito vectors.

In 2010, in the Netherlands, the Centre for Monitoring of Vectors (CMV) started a nationwide inventory of indigenous mosquitoes to acquire basic information on the composition, geographical distribution, biodiversity and environmental preferences of mosquito species. In this survey, the nuisance mosquito species and potential malaria vector Anopheles plumbeus (Stephens, 1828) (Figure 1) was one of the most frequently collected mosquito species (Ibañez-Justicia et al. 2015). This species has been proven to be able to transmit Plasmodium falciparum (Welch, 1897), the causative agent of malaria tropica (Schaffner et al. 2012). Circumstantial evidence for local transmission of P. falciparum malaria by An. plumbeus has been reported for Germany (Kruger et al. 2001). Anopheles plumbeus has also been incriminated as a vector of Plasmodium vivax (Grassi \& Feletti, 1890) (Shute and Maryon 1974, Sokolova and Snow. 2002) and has been proven to be a laboratory vector of West Nile virus (Schaffner et al. 2001). Even though the health care system is likely to rapidly identify malaria patients and thereby prevent the building up of an infectious human reservoir of Plasmodium parasites (Takken et al. 2007), it is important to gain information on the spatial distribution of An. plumbeus, in order to inform the health care system on the areas at risk.

Anopheles plumbeus is a mosquito species commonly found in forests, where larvae are usually found in water in rot-holes of trees with high salinity and deficiency of oxygen (Bradshaw and Holzapfel 1991). They can also be found in containers with stagnant rain water and groundwater, such as tyres, rainwater casks and cemetery vases (Marshall 1938, 
Schaffner et al. 2012). In the last decade, this species has also been associated with abandoned stables where it breeds in the rain water collected in the manure cellars (Dekoninck et al. 2011). This species is known to be a particularly aggressive biter, feeding at any time of the day on different mammalian hosts (including humans), and to a lesser degree on birds and reptiles (Bueno-Mari and Jimenez-Peydro 2012). In June 2006, nuisance caused by An. plumbeus was reported for the first time in the Netherlands, near the city of Nijmegen (Versteirt et al. 2009). Since then, An. plumbeus nuisance have been reported every year in the Netherlands, mostly in proximity to abandoned pig stables (Ibañez-Justicia, unpublished).

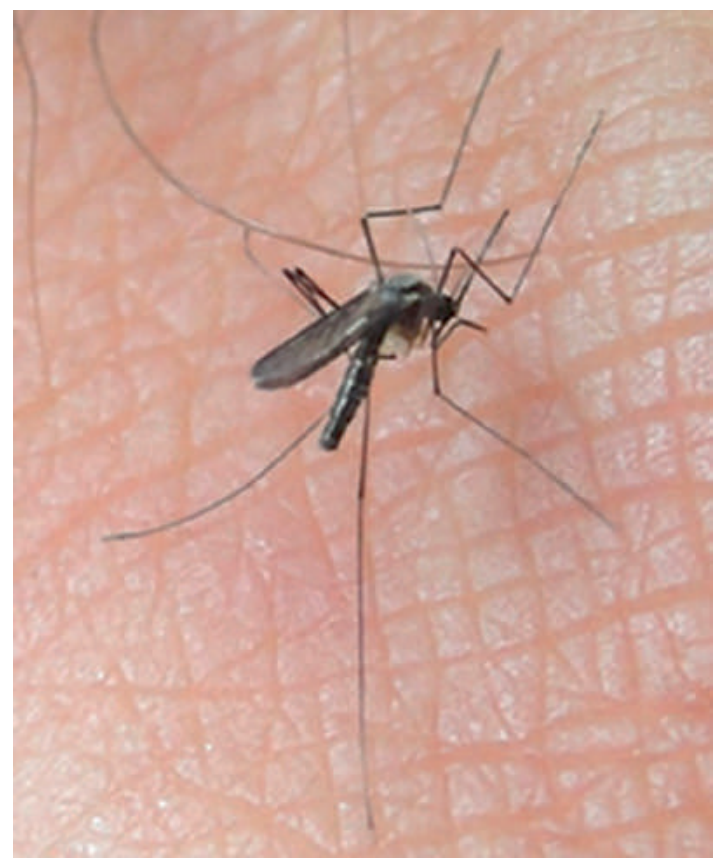

Figure 1. Anopheles plumbeus female (source: A. Ibañez-Justicia).

An understanding of the spatial extent of potential vector species, their abundance and seasonal activity, is important for estimating levels of risks of VBDs and enabling better targeting for surveillance and control. In order to develop basic reproduction number (R0) models and construct risk maps that indicate the risk for an outbreak after an introduction, abundance data of vectors are an essential parameter (Hartemink et al. 2009, Hartemink et al. 2011). Although vector presence and abundance are not the only factors determining whether or not a pathogen can spread in an area, determining the distribution of the vector is an essential step in studying the risk of transmission of a pathogen. Given the nuisance and potential risk for the human health, such information on An. plumbeus is needed. Currently, 
no information on the potential spatial distribution of this species is available for the Netherlands.

In this study, we modelled the potential spatial distribution, expressed in occurrence (predicted probability of presence) and abundance of An. plumbeus in the Netherlands, based on data collected during the National Mosquito Survey and environmental data. The occurrence was modelled to predict the environmental suitability of the species using a random classification forest model. The abundance was modelled using a random regression forest model with the aim to identify areas where mosquito peaks could be expected. Random (classification and regression) forest models allow external validation through a bootstrapping procedure. The occurrence model was validated also with an external dataset. The resulting maps are in agreement with the reported nuisance for this species and the predictions show a good matching with an external dataset used to validate the model.

\section{Methods}

Species distribution modelling links the occurrence or the abundance of species with environmental data and estimates the similarity of the conditions at any site based on the conditions at the locations of known occurrence/abundance of a species. Here we describe the mosquito data collection, the environmental data used and the statistical methods applied in this study.

\section{Mosquito data}

Mosquito data used for the modelling were obtained from the national mosquitoes survey that was carried out from April to October 2010-2013 by the Dutch Centre for Monitoring of Vectors. Mosquitoes were captured using $\mathrm{CO}_{2}$ baited Mosquito Magnet Liberty Plus MM3100 (Woodstream ${ }^{\circledR}$ Co., Lititz, USA). Traps were randomly distributed in the country following a cross-sectional study design, with the following constraint: $40 \%$ of the traps were placed in urban areas, $40 \%$ in rural-agricultural areas and $20 \%$ in natural areas (IbañezJusticia et al. 2015). Urban and agricultural areas were sampled more intensively, because of the potential higher human and veterinary health risk in those areas due to higher exposure.

Data consisted of mosquito abundance data, sampled at 778 locations. For this study the abundance data were reclassified into data of presence (when at least one mosquito was found in the trap) and absence (when no mosquitoes were found in the trap). Each of the locations 
Table 1. Surveys used for the validation. The predictions obtained with the occurrence model that used National Mosquito Survey data were validate with data from these surveys. EMS: Exotic Mosquito Survey. NVS: National Vector Survey.

\begin{tabular}{|c|c|c|c|c|}
\hline Survey name & Year & Sampling strategy & Capturing device & $\begin{array}{l}\text { Total nr } \\
\text { locations }\end{array}$ \\
\hline EMS-Used tires & $2010-2014$ & Target longitudinal sampling & $\begin{array}{l}\text { Larval sampling, } \\
\text { manual aspirator, } \\
\text { BG-Sentinel trap }\end{array}$ & 16 \\
\hline EMS-Lelystad & 2013 & Target sampling & MM-Liberty Plus trap & 3 \\
\hline NVS-Limburg & 2009 & Cross-sectional & MM-Liberty Plus trap & 14 \\
\hline $\begin{array}{l}\text { NVS-Mosquitoes } \\
\text { longitudinal }\end{array}$ & 2011 & Target longitudinal sampling & MM-Liberty Plus trap & 1 \\
\hline Projects & 2011,2012 & Target longitudinal sampling & $\begin{array}{l}\text { CDC light trap, } \\
\text { manual aspirator }\end{array}$ & 3 \\
\hline Nuisance & $\begin{array}{l}2010,2011 \\
2013,2014\end{array}$ & $\begin{array}{l}\text { Check at locations of reported } \\
\text { nuisance }\end{array}$ & $\begin{array}{l}\text { Larval sampling, } \\
\text { manual aspirator }\end{array}$ & 6 \\
\hline $\begin{array}{l}\text { West-Nile-Virus } \\
\text { Wetlands }\end{array}$ & 2010 & Target longitudinal sampling & $\begin{array}{l}\text { MM-Liberty Plus trap, } \\
\text { CDC light trap }\end{array}$ & 2 \\
\hline
\end{tabular}

was sampled only once and each trap was active for one week. The content of the traps was collected weekly and sent to the CMV laboratory. In the laboratory, mosquitoes were morphologically identified using, among others, the Culicidae key specifically designed for rapid field-identification of Dutch adult Culicidae (modified key after (Snow 1990, Schaffner et al. 2001, Verdonschot 2002, Becker et al. 2010)). Twenty-seven mosquito species were found in the National Mosquito Survey and An. plumbeus was the 7th mosquito species most commonly found in the Netherlands. This species was active in the whole period of the survey, from April until October (Ibañez-Justicia et al. 2015).

When a presence and an absence point were in the same square kilometre only the presence point was used because presences inform about the places that are environmental suitable for a species, but absences do not necessarily indicate the opposite (Lobo et al. 2010). This reduced the number of locations used in the analysis from 778 to 766 .

For the validation of the occurrence model, data on An. plumbeus presence from confirmed nuisance notifications and data from other mosquito surveys carried out by the CMV in the Netherlands during the years 2010-2014 were used (Table 1). The mosquito data from these surveys were collected with various trapping methods: dippers, pooters, CDC miniature light traps Model 512 (John W. Hock Company, Gainesville, USA), BG Sentinel traps (Biogents AG, Regensburg, Germany) and Mosquito Magnet traps. These data were extracted from VecBase, a tailor-made application built for CMV in 2010 for vector surveillance data. 
Table 2. Fourier components from temporal Fourier analysis of an imagery time series. Component is the name used in Vecmap.

\begin{tabular}{ll}
\hline \hline Component & Description \\
\hline A0 & Fourier mean for entire time series \\
MN & Minimum value \\
MX & Maximum value \\
A1 & Amplitude of annual cycle \\
A2 & Amplitude of bi-annual cycle \\
A3 & Amplitude of tri-annual cycle \\
VR & Total variance \\
P1 & Phase of annual cycle \\
P2 & Phase of bi-annual cycle \\
P3 & Phase of tri-annual cycle \\
D1 & Proportion of total variance due to annual cycle \\
D2 & Proportion of total variance due to bi-annual cycle \\
D3 & Proportion of total variance due to tri-annual and cycle \\
DA & Proportion of total variance due to all three cycles \\
\hline
\end{tabular}

\section{Environmental variables}

The environmental data included in the analysis as predictor variables are $1 \mathrm{~km}^{2}$ resolution satellite images and meteorological data in raster file format, commonly used for mosquito distribution modelling (ECDC 2013). The images were obtained from the MODIS sensor on NASA's Terra and Aqua satellites (Justice et al. 2000, MODIS 2012) for 2000-2012 and subjected to temporal Fourier transformation (Rogers and Williams 1994, Rogers 2000) to summarise the images and to produce sets of data that capture characteristics of the annual seasonality: the mean, the annual, bi-annual and tri-annual amplitudes and phases, the maxima, minima and variances of the middle infra-red, day and night-time land surface temperature, the enhanced vegetation index and the normalized difference vegetation index signals (Scharlemann et al. 2008). Other environmental data used in this study are precipitation ((WorldClim) [www.worldclim.org] and CMORPH (National Weather Service Climate Prediction Center) [www.cpc.ncep.noaa.gov] 1950-2000), population density (compiled from the Gridded Population of the World Dataset (Socioeconomic Data and Applications Center) [sedac.ciesin.columbia.edu] 2000), the digital elevation model (MODIS 2012) and land cover (Corine land cover map (European Environment Agency) [www.eea.europa.eu] of 2006). The Fourier components used are provided in Table 2 and the environmental data in Table 3. For each trap location the pixel values of the environmental variables were extracted. 
Table 3. Environmental predictor variables

\begin{tabular}{ll}
\hline \hline Source & Variables \\
\hline MODIS & Middle Infra-red (MIR) \\
MODIS & Day-time land surface temperature (DLST) \\
MODIS & Night-time land surface temperature (NLST) \\
MODIS & Enhanced vegetation index (EVI) \\
MODIS & Normalised difference vegetation index(NDVI) \\
CMORPH & Precipitation \\
WorldClim & Precipitation \\
MODIS & Digital elevation model (DEM) \\
Gridded Population of the World & Human population density \\
European Environment Agency & Corine land cover \\
\hline
\end{tabular}

\section{Statistical analysis}

\section{Occurrence model}

Three distribution modelling techniques suitable for occurrence data were applied: non-linear discriminant analysis (Rogers 2000), random classification forest (Breiman 2001) and generalised linear model (McCullagh and Nelder 1989). For each model, the accuracy was assessed using (i) sensitivity, i.e. the ability of a model to correctly identify known positive sites; (ii) specificity, i.e. the ability of a model to correctly identify known negative sites; (iii) the area under the curve, (AUC) that can be roughly interpreted as the probability that a model will correctly predict positive and negative sites (Fielding and Bell 1997). Of the three techniques, random forest provided the best accuracy and therefore the results of this model are presented.

A random classification forest model consists of an ensemble of trees. To create a reliable model, it is generally considered necessary to have the same number of presence and absence points as input. This is because having a different number will create a bias in the model prediction towards the more prevalent category (presence or absence) (Fielding and Bell 1997). For this reason, a 'balanced' subset of the data, i.e., a dataset with the same number of presences and absences, was selected. The output produced by the model is an environmental suitability indicator, expressed as a value between 0 (low suitability) and 1 (high suitability). The predictions are visualised in a map with colours ranging from red (high suitability) to blue (low suitability). A list of the most important variables used in the model is given based on the mean decrease in Gini index (Breiman et al. 1984, Breiman 2001). Random forest allows external validation through a bootstrapping procedure: for each tree, a random subset 
of the full dataset is sampled with replacement. The model validation is carried out for each tree using the points not used from the full dataset. This validation method is referred to as external, because the model is validated using data that are not used to build the tree. The comparison of the observed and predicted results enables us to calculate accuracy statistics, such as sensitivity and specificity. These measures are calculated for each tree and then averaged to give the overall values.

The predictions produced by the random classification forest were also externally validated against 45 observations from other surveys (Table 1) that reported only the presence of $A n$. plumbeus. Comparing the observations obtained with the other surveys and the predictions made by the model using National Mosquito Survey data, the error rate was calculated as the proportion of incorrectly predicted pixels to the total number of points used in the validation.

\section{Abundance model}

The abundance of the species was modelled using a random regression forest model. The abundance data were transformed according to the formula $\log _{10}($ abundance +1 ) (Avia-GIS 2012). Because the aim was to identify areas where mosquito peaks could be expected, only the data collected in months in which peaks were observed were selected (June-September). The predicted environmental suitability obtained with the occurrence model described above, was included as one of the predictor variables for modelling the abundance of the species, as it is frequently done in this type of analysis (Osborne et al. 2001, Boyce et al. 2002, Gibson et al. 2004, Chefaoui et al. 2005, Calvete et al. 2008). The predicted abundance is interpreted as the expected maximum number of mosquitoes caught in a trap in a certain pixel. The predictions are visualised in a map with colours ranging from light green (low abundance) to dark green (high abundance). The importance of the predictors was assessed using the Increase in Node Purity (INP). The difference between observed and predicted values was expressed as the mean squared error. The analysis has been performed with the software Vecmap demo version (Avia-GIS 2014). The maps have been produced with Quantum GIS (QGIS Development Team 2012).

\section{Results and discussion}

The probability of occurrence (environmental suitability) and the abundance of An. plumbeus have been predicted using mosquito field data and environmental data. The estimated environmental suitability and abundance are shown in maps. The important environmental 
variables used in the models and the accuracy of the models are discussed. The fact that out of three different modelling techniques for occurrence data random forest model was selected based upon its higher classification accuracy is consistent with earlier findings; random forest has been reported to outperform other traditional modelling techniques (Cutler et al. 2007, Peters et al. 2007, Cianci et al. 2015).
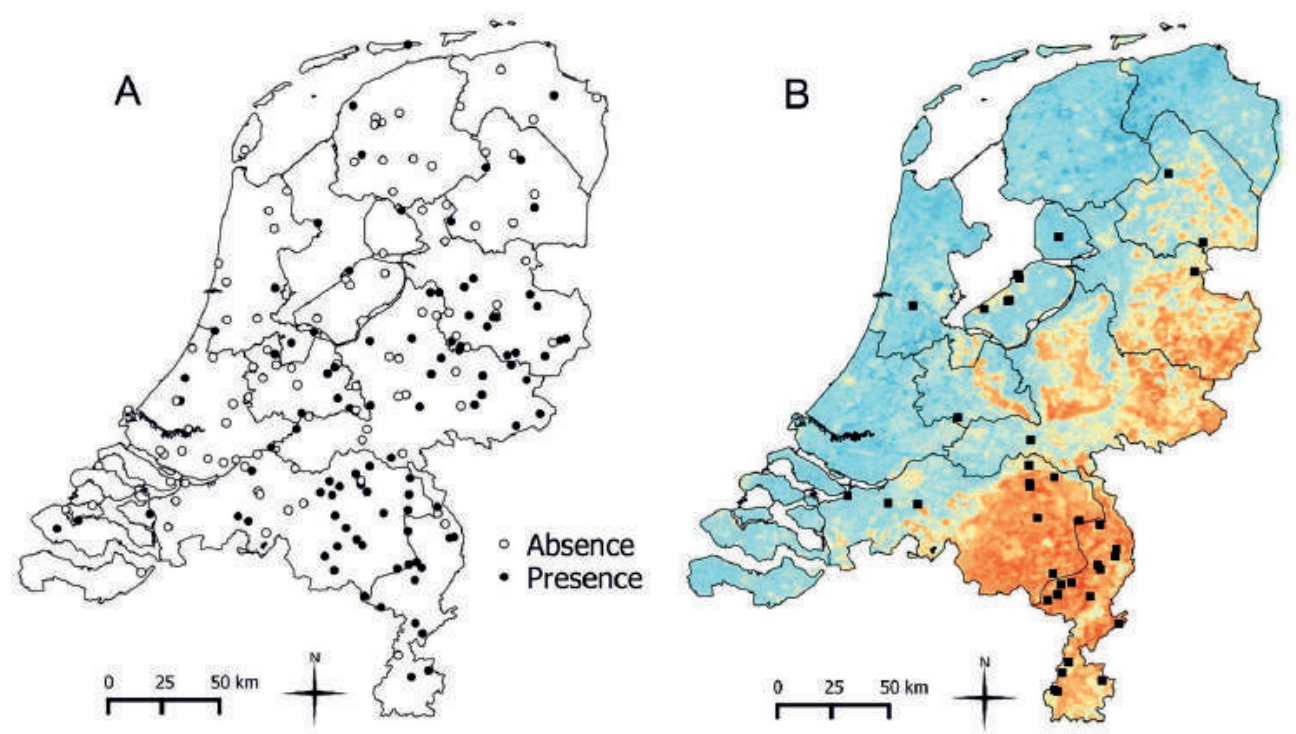

Figure 2. Observed presence and absence points and map of the estimated environmental suitability for An. plumbeus. A- Presence and absence observed during the National Mosquito Survey program carried out from April to October 2010-2013. B- Environmental suitability map of An. plumbeus produced using classification random forest. Environmental suitability is depicted using a gradient fill: blue indicates low environmental suitability, red indicates high suitability. Locations where other surveys took place are also shown on the map (black squares).

An. plumbeus was found in 100 locations out of 766, and it was observed in particular in the eastern part of the Netherlands (Figure 2a). The percentage of traps containing An. plumbeus per week is shown in Figure 3. For the modelling, 97 presence points and 97 absence points were selected. Using the random forest model, forest-rich areas in the centre of the Netherlands (e.g. National Park Hoge Veluwe and National Park Utrechtse Heuvelrug) are predicted as suitable for An. plumbeus (Figure 2b). Also the eastern parts of the country and in particular the southeastern provinces (Limburg and North Brabant) are predicted to be suitable. In these two provinces nuisance is often reported, especially close to abandoned and un-cleaned pig stables, where mosquitoes breed in manure pools (Dekoninck et al. 2011). Based on the environmental characteristics included in the analysis, the model was capable of 
identifying areas where An. plumbeus is truly present, meaning that these characteristics can be a good proxy for abandoned and un-cleaned stables.

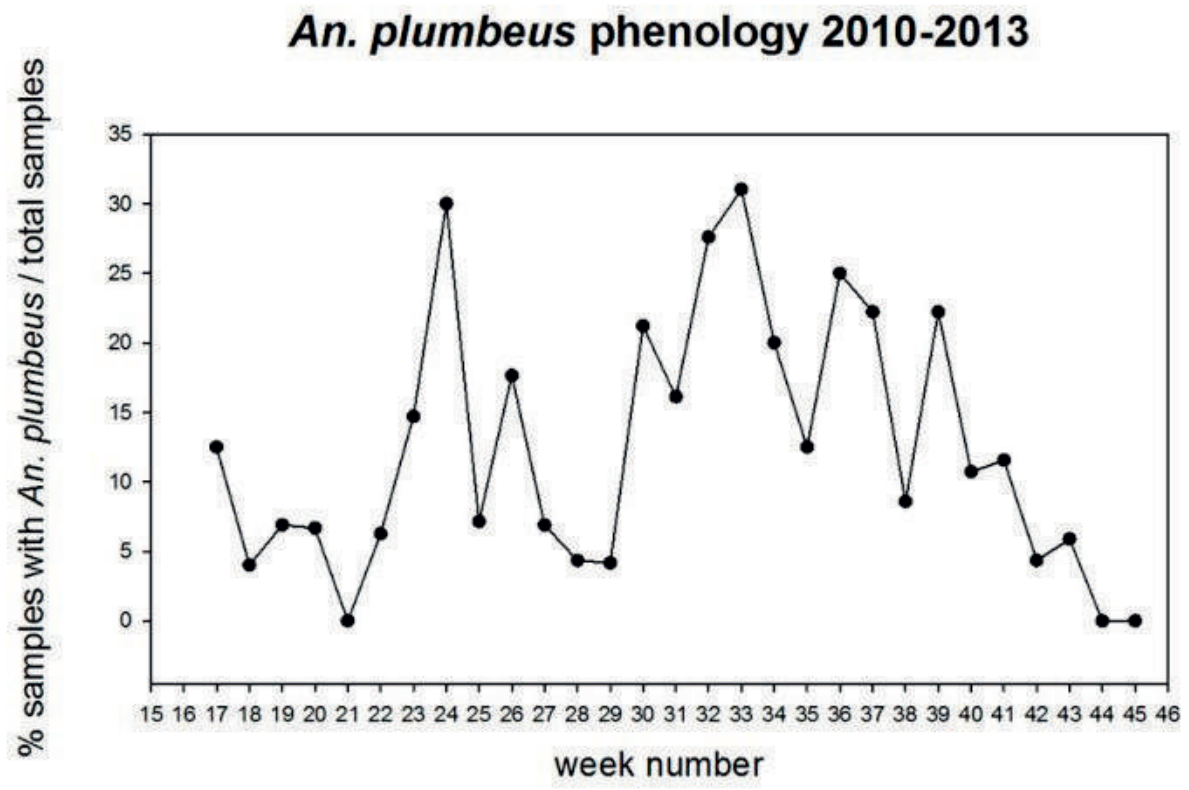

Figure 3. Percentage of positive sites of An. plumbeus per week in 2010-2013.

Elevation, precipitation, day and night temperature, vegetation indices and middle infra-red (index sensitive to changes in the vegetation) were found to be important predictors for environmental suitability for An. plumbeus (Table 4). Precipitation and vegetation are likely to be biologically relevant, since this species typically breeds in water-filled tree holes with high organic material content (Becker et al. 2010). Presence of tree-holes is related to the age of the tree or to the tree species. Eggs of this mosquito species are not laid on the water surface but on the sides of potential breeding sites, just above the waterline, so the number of generations produced each year are often dependent upon hydrological conditions (Becker et al. 2010). The occurrence model predicts environmental suitability for An. plumbeus in areas where old trees with tree-holes are known to occur (e.g., National Park Hoge Veluwe). Even though the species is considered to be a tree-hole breeding species, results obtained using the random forest occurrence model depict environmental suitability for this species in areas without forests in the Netherlands. The results indicate the potential successful use of unforested environments for An. plumbeus populations, and imply a similar trend to that seen in continental Europe and UK, where An. plumbeus is shifting habitats from almost 
exclusively breeding in tree-holes to exploiting a wider array of novel man-made larval breeding sites (Townroe and Callaghan 2014, Ibañez-Justicia et al. 2015). Day and night temperature were found to be related to environmental suitability also in another study in Belgium (Versteirt et al. 2009).

Fair accuracy was obtained with the model $(\mathrm{AUC}=0.73)$, which showed a better ability in identifying suitable environments (sensitivity 0.71) than unsuitable environments (specificity 0.66). The accuracy is improved as compared with a first attempt of predicting the environmental suitability for An. plumbeus in the Netherlands, where the environmental suitability was extrapolated from Belgium to the Netherlands (sensitivity $=0.50$, specificity $=$ 0.49) (Versteirt et al. 2009). The error rate calculated to compare the predicted values to data of other surveys was low (0.29); $71 \%$ of the pixels were correctly predicted, meaning that the model could make good predictions in non-sampled areas. However, this is only a partial validation because it considers only presence points and does not give information about the performance of the model in predicting absence points.

Table 4. List of the top 10 most important variables in the occurrence model. The lowest ranking number indicates the most important variable (e.g., rank $=1$ is the most important variable).

\begin{tabular}{ll}
\hline \hline Rank & Variables \\
\hline 1 & DEM \\
2 & CMORPH precipitation, phase of bi-annual cycle \\
3 & CMORPH precipitation, phase of annual cycle \\
4 & Worldclim precipitation, phase of annual cycle \\
5 & Worldclim precipitation, proportion of total variance due to annual cycle \\
6 & MIR, phase of annual cycle \\
7 & NTLS temperature, minimum value \\
8 & DTLS temperature, amplitude of annual cycle \\
9 & NDVI mean \\
10 & CMORPH precipitation, maximum value \\
\hline
\end{tabular}

The observed and estimated abundance are shown in Figure 4. In the summer, the observed field abundance was low; rarely more than 10 mosquitoes per trap were found (Table 5). The maximum value observed was 1701 mosquitoes followed by 62 mosquitoes per trap. Given the huge difference between the maximum value and the numbers of mosquitoes observed in the other traps, the maximum value was considered as outlier and excluded from the analysis. However, the reason of this high abundance was investigated and it turned out that the trap 
was located in a rural area where cattle farms with abandoned stables, not in use anymore, are present. In total, 505 mosquitoes were used in the analysis and they were captured in 80 traps/weeks from 2010 to 2013. The predicted abundance, produced with the random forest technique, was also low (with a maximum of 15 individuals per trap) and the highest abundance was predicted in the eastern part of the country and especially in the south-eastern provinces Limburg and Brabant. These findings are in agreement with the suitable areas identified by the occurrence model. This is not surprising because the probability of occurrence was the most important factor among the environmental factors included in the abundance model (Table 6). Similar results, where the probability of occurrence appears to be the most important factor when using this technique, were already observed in a study conducted on other mosquito and biting midges species in the Netherlands (Avia-GIS 2012). Other important predictors for abundance of An. plumbeus were precipitation and vegetation, in accordance with the biology of the species, as it is explained above. The difference between the observed abundance and the predicted abundance was small, with a mean squared error value of 0.12 , meaning that the model predictions matched the observation.

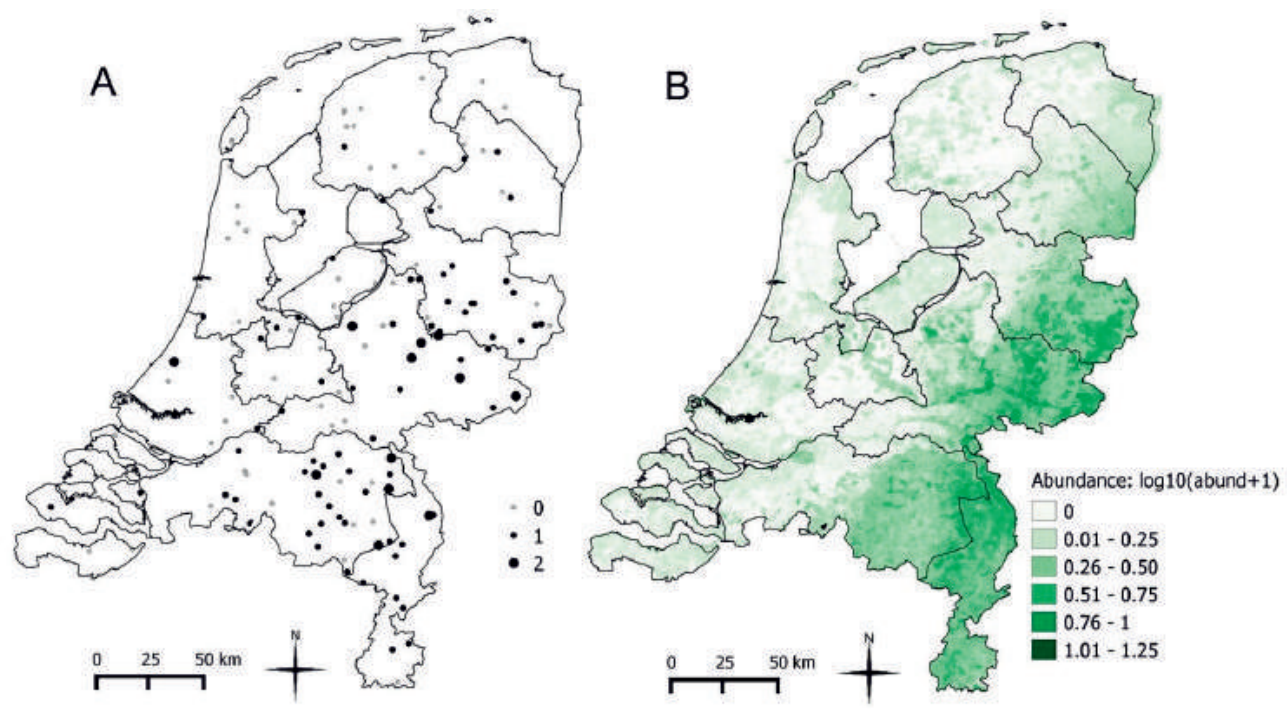

Figure 4. Observed and estimated abundance of An. plumbeus. A - Observed abundance represented as $\log _{10}($ abundance +1$)$. B - Map of the estimated abundance produced using a regression random forest. A darker colour indicates higher abundance. 
Table 5. Observed abundance used in the model

\begin{tabular}{ll}
\hline \hline Count & Frequency \\
\hline 0 & 66 \\
$1-10$ & 70 \\
$11-20$ & 4 \\
$21-30$ & 1 \\
$31-40$ & 2 \\
$41-50$ & 2 \\
$51-60$ & 0 \\
$61-70$ & 1 \\
\hline
\end{tabular}

Table 6. List of the top 10 most important variables in the abundance model. The lowest ranking number indicates the most important variable (e.g., rank $=1$ is the most important variable).

\begin{tabular}{ll}
\hline \hline Rank & Variables \\
\hline 1 & Occurrence \\
2 & Worldclim precipitation, phase of annual cycle \\
3 & Worldclim precipitation, proportion of total variance due to bi-annual cycle \\
4 & NDVI, amplitude of annual cycle \\
5 & Worldclim precipitation, amplitude of bi-annual cycle \\
6 & MIR, amplitude of annual cycle \\
7 & DEM \\
8 & NTLS temperature, phase of bi-annual cycle \\
9 & Worldclim precipitation, total variance \\
10 & CMORPH precipitation, phase of bi-annual cycle \\
\hline
\end{tabular}

\section{Conclusions}

The aim of this study was to investigate the potential spatial distribution of An. plumbeus in the Netherlands. Using random (classification and regression) forest models, we identified areas with high environmental suitability and high abundance of this species in south-eastern provinces of Limburg and Brabant. These areas coincide with the areas where in recent years most nuisances have been reported. The predictions of the occurrence model were accurate and matched the external dataset used for validation. The abundance model predictions also matched the observation.

The output of species distribution modelling method can be used as an input for risk assessment of establishment and spread of vector-borne diseases (Miller 2010, Fischer et al. 2013). Understanding and depicting the potential spatial distribution of mosquito species with modelling techniques is of increasing importance, especially for nuisance mosquito species that can cause economic implications or impact on human health. 


\section{Acknowledgements}

The authors would like to thank Nienke Hartemink and Marieta Braks for a critical reading of the manuscript and useful comments. The authors would also like to thank Els Ducheyne and Wesley Tack for helpful discussion and suggestions. This study was funded by EU grant FP7-261504 EDENext and is catalogued by the EDENext Steering Committee as EDENext311 (http://www.edenext.eu). The contents of this publication are the sole responsibility of the authors and do not necessarily reflect the views of the European Commission. 



\section{Chapter 4}

Pathways for introduction and dispersal of invasive mosquito species in Europe: a review

Adolfo Ibáñez-Justicia

Centre for Monitoring of Vectors (CMV), Netherlands Food and Consumer Product Safety Authority (NVWA), Wageningen, The Netherlands. 


\begin{abstract}
In the last decades, invasive mosquito species (IMS) originating from (sub)tropical regions have arrived to Europe from overseas, introduced via different pathways. Invasive mosquito species such as Aedes albopictus, Ae. japonicus and Ae. koreicus initially passed unnoticed, build populations, and spread in parts of the continent. The introduction and possible establishment of IMS in Europe is a risk for public health, due to the ability of these mosquitoes to transmit vector-borne diseases. The identification of pathways for introduction of IMS is critical to decide on surveillance strategies needed to reduce the risk of future introductions and/or potential outbreaks of mosquito-borne diseases. This chapter reviews the state-of-the-art in our knowledge of pathways associated with the introduction and dispersal of IMS in Europe. Four main routes are identified and discussed: the trade on used tires, the import of Lucky bamboo plants from Asia, the passive transport of IMS in vehicles (traffic by road, airplanes, and sea), and the natural dispersal of IMS. It is concluded that the trade in used tires remains the main pathway for long distance transportation of IMS into and within Europe. Furthermore, passive transport in ground vehicles represents the major driving force for dispersion from already established IMS populations.
\end{abstract}

Keywords: Aedes, Europe, vector-borne diseases, invasive mosquitoes 


\section{Introduction}

In the last decades, invasive mosquito species (IMS) originating from (sub)tropical regions have colonised large areas in Europe due to human trade and transport and helped by suitable climate conditions in Europe. Species such as Aedes albopictus (Skuse, 1895), Ae. japonicus (Theobald, 1901), and Ae. koreicus (Edwards, 1917) have arrived from overseas, introduced by different pathways. Once introduced, they initially passed unnoticed, built populations, and spread across the continent. Following establishment and spread, efforts to control IMS populations or eradicate them are considered extremely difficult if not impossible (Paupy et al. 2009, Baldacchino et al. 2015). The introduction and possible establishment of IMS in Europe is a risk for public health, due to the ability of these mosquitoes to transmit several vector-borne diseases (VBD). This has been demonstrated for Ae. albopictus by outbreaks of dengue and chikungunya in southern France (Gould et al. 2010, La Ruche et al. 2010, Calba et al. 2017), dengue in Croatia (Gjenero-Margan et al. 2010) and chikungunya in Italy (Rezza et al. 2007, Venturi et al. 2017). Similarly, populations of Ae. aegypti have been implicated in a dengue outbreak on Madeira island (Portugal) (Sousa et al. 2012).

It is generally agreed that these outbreaks were only possible because of the presence of established populations of IMS, capable to transmit these VBD. The first sighting of IMS in Europe came from Albania in 1979, when Ae. albopictus was probably introduced with trade of goods from China (Adhami and Reiter 1998). The first Italian observations of Ae. albopictus were reported from Genoa and Padova in 1990 and 1991 (Sabatini et al. 1990, Dalla Pozza and Majori 1992). After the discovery of the introductions, the species started spreading from Italy across southern Europe (Knudsen et al. 1996). Several specific pathways, associated to human trade and transport have been identified in further introductions of IMS in Europe. Trade of used tires has been incriminated for introduction of Ae. albopictus in European countries such as France, Belgium, the Netherlands, Croatia, Montenegro and Spain (Dalla Pozza and Majori 1992, Schaffner and Karch 2000, Petrić et al. 2001, Scholte and Schaffner 2007, Generalitat de Catalunya 2008, Scholte et al. 2010a), as well as Ae.japonicus in Belgium (Versteirt et al. 2009), and Ae. atropalpus and Ae. aegypti in the Netherlands (Scholte et al. 2010a). Trade of Lucky bamboo ornamental plants has also been linked to introduction of Ae. albopictus into the Netherlands and Belgium (Scholte et al. 2008, Demeulemeester et al. 2014). Ground transport from areas heavily infested with Ae. albopictus resulted in the spread of this species from Italy into new areas in Europe such as Spain, Switzerland, the Czech Republic, and Germany (Aranda et al. 2006, Scholte and 
Schaffner 2007, Sebesta et al. 2012, Kampen et al. 2013). It is also expected that the continuous trade and global tourism will promote further accidental introductions of IMS (e.g. Ae. albopictus) into Europe and also beyond regions where they have already established (Cunze et al. 2016a).

In order to monitor the spread of IMS and identify new introductions, the VBORNET and VectorNet projects established a European network of entomological and public health specialists in order to assist the European Centre for Disease Prevention and Control (ECDC) and the European Food Safety Agency (EFSA) in its preparedness activities on VBDs. These programmes provide information needed for risk assessment. As a result, one of the outputs of these programmes are the quarterly VectorNet maps showing the currently known distribution of invasive species in Europe at 'regional' adm inistrative level (NUTS3) (ECDC \& EFSA 2018), and the mosquito factsheets for experts (ECDC 2017). Furthermore, the ECDC produced guidelines to support the implementation of IMS surveillance in Europe (ECDC 2012).

In 2012, Medlock et al. published a review of IMS in Europe including an overview of the importation routes of the exotic aedine mosquitoes established or intercepted in European countries (Medlock et al. 2012). Nowadays, in 2018, IMS have expanded their distribution in Europe, including new countries, and they have been associated to new pathways for introduction and dispersal. The main objective of this review is to compile information on known and suspected pathways for introduction and dispersal of IMS, and discuss their relative importance in Europe. An exhaustive review identifying the pathways is lacking, and the evaluation of their importance at the European level will provide a "check list" for monitoring activities on IMS surveillance in Europe. This "check list" can be used by monitoring teams and decision makers at national and regional level within Europe to decide on surveillance strategies needed to reduce the risk of future introductions and/or potential outbreaks of mosquito-borne disease.

\section{Overview of pathways for introduction and dispersal of invasive mosquitoes in Europe}

Trade of used tires 
The global trade and transport of used tires is considered as the greatest risk for importation of invasive mosquitoes. Water that collects in tires stored outdoors provides excellent mosquito breeding sites. Exposed to the elements, this water accumulates organic material and microbes that are used as larval nutrition (Yee 2008). In addition such artificial breeding sites offer high humidity, high temperatures, and a lack of natural enemies. Female aedine invasive species lay their eggs in these tires, and when tires are transported, tires serves as a 'vehicle' to accidentally transport IMS eggs to new areas (Reiter and Sprenger 1987). These eggs are drought-resistant and therefore able to withstand prolonged periods without water when tires are transported to other countries or continents. When the water level inside tires rises due to rain (e.g., in a new area where that species did not previously occur) the eggs can hatch. Adult mosquitoes that originate from such breeding sites can subsequently invade natural water bodies near the site where the tires were stored, and initiate new, viable mosquito populations.

The trade in used tires has been the principal hypothesis as introduction pathway for $A e$. albopictus from endemic areas in Asia to the United States (Hawley et al. 1987). After the arrival and establishment of Ae. albopictus in U.S. from Asia, the species also expanded in the country primarily via the transport of eggs in used tires (Hawley et al. 1987), and using the interstate highway networks as a major expansion pathway (Medley et al. 2014; Moore \& Mitchell 1997). The transport of used tires was also confirmed as the pathway for introduction of Ae. japonicus in New Zealand, when larvae were found in imported used tires from Japan (Derraik 2004).

The discovery of Ae. albopictus in Albania in 1979 and Italy in 1990 (see above) was associated to the trade on used tires. However, it was only after the introduction in Italy when the species spread quickly and it was considered a threat to public health (Knudsen et al. 1996). Trade of used tires has been incriminated in the introduction of Ae. albopictus in countries such as France, Belgium, Croatia, Montenegro, Spain, the Netherlands, and recently in Portugal (Schaffner and Karch 2000, Petrić et al. 2001, Scholte and Schaffner 2007, Generalitat de Catalunya 2008, Scholte et al. 2010a, Osorio et al. 2018).

Using this pathway, the North American species Ae. triseriatus (Say, 1823) was intercepted in France in 2004 when it was introduced with used tires originating from Louisiana (USA) (S. Chouin \& F. Schaffner, unpublished data in (Medlock et al. 2012)). Similarly, Ae. japonicus has been also detected in used tire companies in Belgium (Versteirt et al. 2009) and in The Netherlands (in this thesis). In Belgium, larvae were collected in consecutive years 
from the used tires, and also early in the season (March) indicating overwintering of the species at these locations. Surprisingly, no evidence of spread of this population was found in the surrounding area (Versteirt et al. 2009).

The finding of Ae. atropalpus in 2009 at two companies which import used tires into the Netherlands demonstrated that also this country is exposed to the risk of IMS imported via the international used tire trade. Prior to 2009, there are no reports of IMS imported into the Netherlands via used tires, although data collected in 2009 and 2010 suggest that it could have happened before the first reported finding (Scholte et al. 2009). In Europe, the same species was found in Italy in 1996 (Romi et al. 1997) and in France in 2003 and 2005 (S. Chouin and F. Schaffner, unpublished data in (Medlock et al. 2012)), through import of used tires. Following the mosquito control applied to control the populations in the Netherlands, Ae. atropalpus was no longer detected at any of the sites.

In 2010, Ae. aegypti was detected for the first time in the Netherlands at two used tire companies (Scholte et al. 2010a). Back-tracing data of the companies suggests the introduction of the species by a shipment of used airplane tires at the end of May 2010, originating from southern Florida, an area where the species is widely present (Scholte et al. 2010a). Additionally, in a study supported by VectorNet in the Eastern Black sea area in Turkey (Akiner et al. 2016), both Ae. albopictus and Ae. aegypti were found from 2015 to 2016 in used tire storage areas.

\section{Trade of Lucky bamboo (Dracaena sp.) plants}

Dracaena sanderiana (Sparagalus: Dracaenaceae [Agavaceae]), an ornamental plant species known as 'Lucky bam boo', is a popular ornamental plant in Europe. These plants are imported from southern China and Taiwan, usually by ship, and they are transported in Perspex boxes containing a few centimetres of water or with gel protecting them from desiccation during transport. Long-distance spread of Ae. albopictus has been linked to the importation of this plant. In 2001, Ae. albopictus was reported in California (USA) associated to import of Dracaena sanderiana plants (Linthicum et al. 2003, Madon et al. 2003) and linked to sea-trailer import of these plants from southern China. The mosquitoes reported on the nurseries had probably developed from eggs that had been deposited near the water level, either on the plants or the Perspex boxes, at the moment the plants were still in southern China. The Netherlands is an important trading country for flowers and plants and the 
principal port of entry of Lucky bamboo plants in Europe. After the finding of Ae. albopictus specimens in a Lucky bamboo nursery in the Netherlands in 2005 (Scholte et al. 2007), it was proposed that transporting the plants on gel rather than in water would reduce the presence of Ae. albopictus adults in Lucky bamboo shipments. However, $70 \%$ of the Dutch nurseries where adult $A e$. albopictus were found in later inspections, claimed to have imported plants on gel only (Scholte et al. 2008). Furthermore, in 2013, a live Ae. albopictus larva was detected on a gel substrate being the first direct evidence of the importation of this species on gel-transported Lucky bamboo (Demeulemeester et al. 2014). Fortunately, these populations found at the nurseries in Europe have not further spread beyond the nurseries, even though on two occasions, there was evidence that the species had escaped from the glasshouses in the Netherlands (Scholte et al. 2008). This fact suggests that these populations are unable to develop under temperate conditions and are thus likely to originate from the tropical strain of Ae. albopictus (Medlock et al. 2012).

At the end of 2013 the IMS Ae. japonicus was identified associated with the import of Lucky bamboo in the Netherlands (see Chapter 6 in this thesis). This finding represents the first associated Ae. japonicus import with this plant elsewhere in the world. Aedes japonicus, similar to Ae. albopictus, is native to southern China (Tanaka et al. 1979) and has a similar oviposition behaviour by laying eggs in bamboo stumps (Sota et al. 1994). This may explain the presence of Ae. japonicus in the imported plants from southern China.

\section{Passive transportation by aircraft}

Due to globalization and increased air connectivity of major cities, air travel has been considered to play a major role in long-distance dispersal of vectors as well as their associated diseases (Gezairy 2003, Tatem et al. 2006). In areas with high mosquito densities, there is a greater probability that mosquitoes can enter aircrafts at the airports following their human hosts (Gratz et al. 2000). These aircrafts can then transfer mosquitoes to another country rapidly, thus increasing the chance of mosquitoes surviving the trip and reaching a location where the mosquitoes are non-native (Tatem et al. 2006). The transport of mosquitoes on aircraft may be more common than expected. A 13-year study from 19471960, where mosquitoes were collected from incoming international flights in the USA, yielded a total of more than 20,000 mosquitoes, belonging to 92 different species (Hughes 1961). 
Upon arrival, mosquitoes may colonize new areas and, if they travel with a disease, even infect people living in the direct vicinity of airports (Gratz et al. 2000) (Bataille et al. 2009). For example, 'airport malaria' refers to malaria caused by infected mosquitoes that are transported rapidly by aircraft from a malaria-endemic country to a non-endemic country. If the local conditions allow their survival, they can bite local residents who can thus acquire malaria without having travelled abroad (CDC 2015). Prior to August 1999, a total of 83 cases of 'airport m alaria' were reported in Europe (Gratz et al. 2000). Similarly, cases of dengue fever acquired at the airport have been reported. In 2010, a mosquito which alighted from Bali with a military aircraft was considered the most likely source of a case of "airport dengue" in Australia (Whelan et al. 2012).

In Europe, mosquitoes have also been introduced from abroad with airplanes. In a French study (Giacomini et al. 1995) it was estimated that 8-20 Anopheles mosquitoes were imported into France on each flight from Africa. In 2008, the Netherlands reported live mosquitoes on a flight from Tanzania to Schiphol airport in Amsterdam, with several passengers complaining of being bitten on board (Scholte et al. 2010b). A follow-up study in Schiphol showed that exotic mosquitoes were transported on 10 of the 38 aircrafts inspected (Scholte et al. 2014). In 2016, adult Ae. aegypti mosquitoes were captured indoors and outdoors at the airport of Schiphol in the Netherlands (Ibañez-Justicia et al. 2017), and in 2017 Ae. aegypti and Ae. albopictus were also found at the airport (NVWA 2018) confirming, for the first time, air transport of these mosquito vector species in Europe. Another invasive mosquito species, Ae. koreicus, was found in 2015 inside the premises of the international airport of Genova (EMCA 2017). No relationship was established with accidental aircraft mediated transport, but the presence of an invasive mosquito species at the airport, nearby an important commercial port is worrisome for a potential further spread of this IMS via aircrafts or boats in Italy and beyond.

\section{Passive transportation: ground}

Intercontinental jumps by IMS are mainly driven by unintentional mass transportation of desiccation-resistant eggs, together with imported products. However, in the newly colonized areas such as in the USA or in Europe, dispersal of İMS has been related to ground transport. For instance, after arrival in the USA, analysis reveals that Ae. japonicus, the most recent mosquito to become globally invasive, has continued to exploit humans as their main mode 
of travel, being their presence associated to their proximity to roads (Egizi et al. 2016). In Europe, it has been hypothesized that Ae. japonicus individuals could have been transported by vehicles along the motorways between the German federal states (Zielke et al. 2015). This assumption is supported by the fact that the initial findings of Ae. japonicus in northern Germany were concentrated in cemeteries of towns close to a motorway running northwards from West Germany (Werner and Kampen 2013).

Passive dispersal of adults of Ae. albopictus in private vehicles could partly explain the rapid spread of the species within European countries. This has been a likely mode of transportation to the Czech Republic from southern Europe in 2016 and 2017 (Rudolf et al. 2018). From an annual survey in Switzerland it became evident that the European motorway E35 running from Rome, Italy, to Amsterdam, the Netherlands, is a key route of invasion of Ae. albopictus into northern Europe (Flacio et al. 2016). In the same survey, another IMS, Ae. koreicus was detected in parallel with Ae. albopictus and seemed to have similar introduction pathways (Suter et al. 2015). A systematic evaluation of service stations along different main motorways in southern Germany demonstrated repeated introductions of Ae. albopictus into Germany, probably originating from established source populations in southern Europe, and introduced by transit road traffic (Pluskota et al. 2008, Werner et al. 2012, Becker et al. 2013). An example confirming that vehicle transport of Ae. albopictus is an important and probably frequent mode of importation is the findings of this species in the city of Freiburg in the Upper Rhine valley (Kampen et al. 2013, Becker et al. 2017). Here, it was proven that the population of mosquitoes is spatially restricted to the allotment garden area, which is adjacent to a train station where trucks from Novara, Italy, arrive by rail (Becker et al. 2017). The recent northernmost findings of Ae. albopictus in Germany, and reported by citizens, are also probably attributed to vehicle introduction from the south (Walther et al. 2017). In the United Kingdom, owing to the existence of Ae. albopictus in continental Europe, and its fast spread in the last years through the French highway systems, a network of mosquito traps was arranged for the detection of the arrival of the species. This trapping network was implemented at arrival locations of vehicles from continental Europe (e.g. ferry ports, Eurotunnel). In 2016, eggs of Ae. albopictus were detected in one ovitrap placed in a lorry park at Folkestone service station (England), close to the Eurotunnel (Medlock et al. 2017). The authors conclude that the finding of eggs in one ovitrap was not evidence of establishment nor an elevated disease risk, and it is probably indicative of an individual female mosquito having entered the UK in a vehicle from continental Europe. 
The first evidence of the role of passive transportation of Ae. albopictus in private vehicles was obtained in an experimental study performed in Catalonia, Spain. In this study, in collaboration with the Catalan Road Service Department, cars were stopped and inspected for the presence of Ae. albopictus, and also vehicles were sampled at Technical Inspection Stations. The study concluded that passive transportation in vehicles is a major driving force for Ae. albopictus dispersion in Europe (Eritja et al. 2017).

\section{Passive transportation: maritime sea traffic transport}

The most relevant example of maritime sea traffic transport of IMS and further spread in other areas, is the historical movement of Ae. aegypti in ships. The species was likely introduced to the New World from Africa by slave trade ships between the fifteenth and eighteenth centuries, possibly on multiple occasions (Tabachnick 1991, Brown et al. 2014). Furthermore, in the 19th century Ae. aegypti was repeatedly introduced into ports by vessels and was widely established in southern Europe (Schaffner and Mathis 2014).

Nowadays, the high number of containers and goods transported by ship, and the high number of direct ferries carrying vehicles with tourists from locations colonized by $A e$. albopictus on the European mainland, are attributed as the main factors for introduction and dispersal of Ae. albopictus to Mediterranean islands and northern Africa. In this case, introduction could be possible by unintentional mass transportation of desiccation-resistant eggs, together with imported goods, and with the passive dispersal of adults of Ae. albopictus in private vehicles carried by the ferries. Using this pathway, Ae. albopictus has been reported to arrive and colonize Greek islands (Samanidou-Voyadjoglou et al. 2005), Malta (Buhagiar 2009), Majorca (Miquel et al. 2013), Ibiza (Barceló et al. 2015), Minorca (Bengoa et al. 2016) and Thyrrhenian islands (Toma et al. 2017). In North Africa, an entomological survey was conducted in 2015 in the town Ain Turk (West of Algeria) to determine the origin of the mosquito nuisance. This revealed the presence of Ae. albopictus in the town. The intense maritime traffic of travellers and goods were considered as the main routes of its introduction in the area (Benallal et al. 2016). Probable introduction using ports is also suspected in the findings of Ae. albopictus on the Sicilian islands of Lampedusa, Linosa and Pantelleria, representing the Southernmost European limit of the species (Di Luca et al. 2017). 


\section{Natural dispersal}

The flight of mosquitoes is influenced by factors such as oviposition site availability, climate (e.g., wind, humidity, temperature, rainfall), terrain, vegetation, housing characteristics and blood source (Forattini 1962, in Honorio et al. (2003)). Active migration of mosquitoes plays a minor role over long distances (Egizi et al. 2016). Specially, invasive Aedes species have a relatively low capability for active dispersal over long distance. Females of Ae. albopictus are only capable of autonomous dispersal over short distances (Hawley 1988, Liew and Curtis 2004). For example, in Missouri, USA, non-blood fed Ae. albopictus females marked with fluorescent dyes dispersed up to $525 \mathrm{~m}$ (female) and $225 \mathrm{~m}$ (male) (Niebylski and Craig 1994). In a study in a dengue endemic area, the dispersal of Ae. aegypti and Ae. albopictus was measured over a distance of at least $800 \mathrm{~m}$ within a six day period (Honorio et al. 2003). Furthermore, results of an extensive literature survey showed that in comparison with other Aedes species (e.g. Aedes vexans 5,727m), the average maximum flight distances of Ae. albopictus $(676 \mathrm{~m})$ and Ae. aegypti $(333 \mathrm{~m})$ are considered weak and very weak respectively (Verdonschot and Besse-Lototskaya 2014).

The autonomous dispersal capabilities of Ae. japonicus appear also limited as evidenced by its failure to expand in Belgium from known distributions sites (Damiens et al. 2014). However, the species has been reported to have a moderate dispersal capability in comparison with other species (Verdonschot and Besse-Lototskaya 2014) with an average maximum flight distance of $1,600 \mathrm{~m}$. Furthermore, it has been proposed that Ae. japonicus adults may autonomously disperse along stream corridors, since this species often deposits eggs in rock pools along streams when they are available (Bevins 2007). The natural dispersal of $A e$. japonicus is suggested as the pathway for introduction in new forested regions in BadenWüttemberg, southwest Germany (EMCA 2017), the introductions in Austria from Germany (Seidel et al. 2016a), and the introductions in Italy from Austria (Seidel et al. 2016b). Since its detection in Western Germany in 2012 (Kampen et al. 2012), the annual geographic expansion of the population has been monitored. Results showed an important increase of the colonised area to more than $8,000 \mathrm{~km}^{2}$ in 2015 (Kampen et al. 2016), probably resulting from a combination of natural spread and human aided transportation. The invasion in Slovenia showed similar patterns to that in West Germany (Kalan et al. 2017). Since the first detection of Ae japonicus close to the Austrian border, the population spread across the border to Croatia, and by 2015 it was present almost throughout Slovenia (Kalan et al. 2017). In the Netherlands, the detection of an established population in a municipality (Lelystad) at more 
than 100 and $150 \mathrm{~km}$ from the Belgium and German borders respectively was surprising (Ibañez-Justicia et al. 2014). Natural dispersal of the species from neighbouring populations in Belgium or Germany was not considered likely due to the absence of the species during monitoring activities in surrounding municipalities contiguous to Lelystad (Ibañez-Justicia et al. 2018a).

\section{Discussion}

This literature review reveals that the main pathways for introduction and dispersal of IMS in Europe are the trade of used tires, the import of Lucky bamboo plants, the passive transportation in vehicles (by ground, sea, air) and the natural dispersal of the IMS. Since the review of Medlock et al. in 2012, new pathways for introduction and dispersal of IMS in Europe have been added. For example, introductions with aircrafts (Scholte et al. 2014, Ibañez-Justicia et al. 2017), with maritime sea traffic in European islands (Miquel et al. 2013, Barceló et al. 2015, Bengoa et al. 2016, Di Luca et al. 2017, Toma et al. 2017), new detections in greenhouses with Lucky bamboo imported from Asia (Demeulemeester et al. 2014, Ibañez-Justicia et al. 2018b), and the movement of IMS with ground vehicles (Becker et al. 2013, Kampen et al. 2013, Flacio et al. 2016, Becker et al. 2017, Eritja et al. 2017, Rudolf et al. 2018). This review points the trade in used tires as one of the greatest risks for long distance transportation of IMS into and within Europe, and the passive transport of IMS in ground vehicles as the major driving force for dispersion within Europe.

Based on the reviewed literature, it can be concluded that the global and national transportation of used tires represents one of the greatest risks for long distance importation of IMS into and within Europe. Several IMS, including Ae. albopictus, Ae. atropalpus, Ae. japonicus, Ae. triseriatus and Ae. aegypti, have been detected using this pathway (see Table 1). This fact stresses the importance of the used tire pathway for introducing a broader range of IMS. Furthermore, the transport of used tires has been confirmed as the main source of introduction of Ae. albopictus in the Netherlands, with yearly detections at companies that import used tires since 2010 .

Depending on the demand in the market, different volumes of containers with Lucky bamboo plants from southern Asia are imported and maintained in Europe. To date, the role of Lucky bamboo for introducing IMS has only been confirmed in The Netherlands and in Belgium where this activity has been identified and it is subjected to monitoring. Since the first 
detection in 2005, the number of Ae. albopictus reports in The Netherlands has been considerably reduced due to the use of hydroscopic gel for transport of plants, and the application of mosquito larvicides in the water where the plants are maintained (in this thesis). Due to the origin of the plants imported, it is likely that the introduced specimens belongs to the Asian tropical strain of Ae. albopictus. This would present a lower probability for adapting to the colder winters in the Netherlands or Belgium. However, this Asian tropical strain could be a risk if introduced with Lucky bamboo plant at suitable areas in southern Europe.

The natural spread of IMS, but in combination with possible human aided transport, has only been suggested as the pathway for spread populations of Ae. japonicus to new areas in Western Europe. Instead, the greatest concern in Europe is represented by the association of IMS dispersal to passive ground transportation (e.g. cars, trucks, train). Aedes albopictus threats to colonize new areas northwards in Western Europe, mainly due to hitchhiking behaviour in cars and trucks. This threat is especially relevant for areas adjacent to established populations of the species, and well connected by roads. The maritime traffic transportation has played an important role to introduce the IMS Ae. albopictus from colonized coastal areas in Europe, to almost all the islands in the Mediterranean sea. Thus far, Ae. albopictus is the only IMS that has been recently introduced and dispersed with maritime traffic transport. In addition, repeated introductions of IMS specimens at the international airport of Schiphol in The Netherlands has shown that transportation of IMS in aircrafts is possible in Europe (Ibañez-Justicia et al. 2017). The substantial number of mosquito species introduced via aircrafts into European countries indicates that accidental introductions are not unusual. In comparison with other pathways that can transport large numbers of mosquito eggs (e.g. used tires), this can be considered a less likely means of establishment due to the low number of individuals potentially introduced inside the aircrafts (Lounibos 2002, Scholte et al. 2010b). However, due to the increase of air-travel and the possibility of repeated undetected introductions of IMS individuals, this could increase the risk of establishment of IMS species. Therefore, it is strongly recommended that European regions implement surveillance at these points of entry (ports and airports) to warn for the introduction of IMS that have been implicated as vectors of disease.

Although clear evidence is lacking, the import of plants other than Lucky bamboo has been suggested as a pathway for introduction into Europe in two cases Aedes koreicus was first detected in 2011 in the locality of Belluno, Italy, during a routine monitoring for Ae. 
albopictus (Capelli et al. 2011). In 2012 it was already found in 37 municipalities (Montarsi et al. 2013). According to the authors (F. Montarsi, pers.comm), the route of entry has not been traced to date. The hypothesis is that Ae. koreicus could have been introduced with the trade of ornamental plants because of the small number of tire traders, and the large amount of garden centres in the area of the first finding. Moreover, plant importers in the area trade with companies that import ornamental plants from The Netherlands and Belgium. In latter country, the first finding of this species was reported in Europe. The import of plants (other than Lucky bamboo) was also suggested as the pathway for Ae. albopictus introduction in 2017 in the Netherlands. An adult specimen of the species was caught in a trap inside the premises of one of the largest flower auctions in Europe (NVWA 2017). Introduction of larvae or pupae in buckets within the thousands of fresh flowers that are imported daily from south European areas could not be ruled out in this case.

In recent years, other discoveries of breeding invasive mosquito populations have also occurred in Europe, with no apparent link to a known pathway. Examples are the discovery of Ae. albopictus (NVWA 2016, 2018) and Ae. japonicus (Ibañez-Justicia et al. 2014) in Dutch urban and peri-urban areas, the uneven dispersal of Ae. albopictus in regions in Spain (Roiz et al. 2007, Collantes et al. 2015, Delacour et al. 2015), the finding of Ae. aegypti on the Canary Islands (Spain) (ECDC 2018b), and the findings of Ae. koreicus in Belgium, Russia, Switzerland, Hungary and Germany (Versteirt et al. 2012, Bezzhonova et al. 2014, Suter et al. 2015, Kurucz et al. 2016, Werner et al. 2016). The current hypothesis for the presence of these populations is human aided transport of the founders of the new populations by air, sea or road. However, the origin of the introductions is not known yet by the authors. The introduction and dispersal of IMS in Europe is a risk for public health and the identification of their pathways of movement within regions is pivotal to decide on surveillance strategies needed to reduce the risk of future introductions and/or potential outbreaks of mosquito-borne diseases.

\section{Conclusion}

The importance of pathways for introduction and dispersal of IMS has changed since several IMS have nowadays established populations in large areas in Europe (e.g. Ae. albopictus in Italy, Aedes japonicus in Germany). Pathways for first introductions in new areas seem nowadays less associated to the "usual suspects" such as used tires or Lucky bam boo, but 
more associated to other human activities (international or domestic travel/trade). In this review, four main routes for introduction of invasive mosquito species were identified: the trade in used tires, the import of Lucky bamboo plants from Asia, the passive transport of IMS in vehicles (traffic by road, airplanes, and sea), and the natural dispersal of IMS. The trade in used tires still represents one of the greatest risks for long distance transportation of IMS into and within Europe. However, the passive transport of IMS in ground vehicles, represents a major driving force for dispersion in Europe. Identification of potential other pathways for introduction of IMS, and evaluating the importance of the different pathways are critical for designing surveillance strategies to promptly detect and control introductions in non-infested areas. 
Table 1. Overview of pathways for introduction and dispersal of invasive mosquito species reported in Europe.

\begin{tabular}{|c|c|c|c|c|c|c|}
\hline Pathway & Species & Country (Region) & Year & $\begin{array}{l}\text { Later } \\
\text { establishment? }\end{array}$ & $\begin{array}{l}\text { Later } \\
\text { spread? }\end{array}$ & References \\
\hline \multirow[t]{3}{*}{$\begin{array}{l}\text { Lucky } \\
\text { bamboo }\end{array}$} & \multirow[t]{2}{*}{ Aedes albopictus } & The Netherlands & 2005 & No & No & $\begin{array}{l}\text { (Scholte et al. 2008, } \\
\text { Hofhuis et al. 2009) }\end{array}$ \\
\hline & & Belgium & 2013 & No & No & $\begin{array}{l}\text { (Demeulemeester et al. } \\
\text { 2014) }\end{array}$ \\
\hline & Aedes japonicus & The Netherlands & 2013 & No & No & $\begin{array}{l}\text { (Ibañez-Justicia, } \\
\text { unpublished data) }\end{array}$ \\
\hline \multirow[t]{18}{*}{ Used tires } & \multirow[t]{2}{*}{ Aedes aegypti } & The Netherlands & 2010 & No & No & (Scholte et al. 2010a) \\
\hline & & Turkey (Black Sea Region) & 2016 & Unknown & Unknown & (Akiner et al. 2016) \\
\hline & \multirow[t]{3}{*}{ Aedes atropalpus } & Italy (Veneto) & 1996 & Yes & No & (Romi et al. 1997) \\
\hline & & France & 2003 & No & No & (ECDC 2014c) \\
\hline & & The Netherlands & 2010 & No & No & (Scholte et al. 2010a) \\
\hline & \multirow[t]{10}{*}{ Aedes albopictus } & Albania & 1979 & Yes & Yes & $\begin{array}{l}\text { (Adhami and Reiter } \\
\text { 1998) }\end{array}$ \\
\hline & & Italy & 1991 & Yes & Yes & $\begin{array}{l}\text { (Dalla Pozza and Majori } \\
\text { 1992) }\end{array}$ \\
\hline & & France & 1999 & No & No & $\begin{array}{l}\text { (Schaffner and Karch } \\
2000)\end{array}$ \\
\hline & & Belgium & 2000 & No & No & (Schaffner et al. 2004) \\
\hline & & Montenegro & 2001 & Yes & Yes & (Petrić et al. 2001) \\
\hline & & Croatia & 2005 & Yes & Yes & $\begin{array}{l}\text { (Scholte and Schaffner } \\
\text { 2007) }\end{array}$ \\
\hline & & Spain (Lleida) & 2008 & No & No & $\begin{array}{l}\text { (Generalitat de Catalunya } \\
\text { 2008) }\end{array}$ \\
\hline & & The Netherlands & 2010 & No & No & (Scholte et al. 2010a) \\
\hline & & Turkey (Black Sea Region) & 2016 & Yes & Unknown & (Akiner et al. 2016) \\
\hline & & Portugal & 2018 & Unknown & Unknown & (Osorio et al. 2018) \\
\hline & \multirow[t]{2}{*}{ Aedes japonicus } & Belgium & 2002 & Yes & No & (Versteirt et al. 2009) \\
\hline & & The Netherlands & 2013 & No & No & $\begin{array}{l}\text { (Ibañez-Justicia et al. } \\
\text { 2018b) }\end{array}$ \\
\hline & Aedes triseriatus & France & 2004 & No & No & (ECDC 2014b) \\
\hline \multirow{11}{*}{$\begin{array}{l}\text { Passive } \\
\text { transport } \\
\text { ground } \\
\text { vehicles }\end{array}$} & \multirow[t]{11}{*}{ Aedes albopictus } & Czech republic & 2012 & No & No & $\begin{array}{l}\text { (Sebesta et al. 2012, } \\
\text { Rudolf et al. 2018) }\end{array}$ \\
\hline & & Germany & 2013 & Yes & No & $\begin{array}{l}\text { (Kampen et al. 2013, } \\
\text { Becker et al. 2017) }\end{array}$ \\
\hline & & Germany & 2008 & No & No & (Pluskota et al. 2008) \\
\hline & & Germany & 2011 & No & No & (Werner et al. 2012) \\
\hline & & Spain & 2006 & Yes & Yes & (Aranda et al. 2006) \\
\hline & & Spain & 2016 & Yes & Yes & (Eritja et al. 2017) \\
\hline & & Switzerland & 2003 & Yes & Yes & (Flacio et al. 2016) \\
\hline & & Germany & 2012 & No & No & (Becker et al. 2013) \\
\hline & & England & 2016 & No & No & (Medlock et al. 2017) \\
\hline & & Montenegro & 2001 & Yes & Yes & $\begin{array}{l}\text { (Scholte and Schaffner } \\
\text { 2007) }\end{array}$ \\
\hline & & Slovenia & 2013 & Yes & Yes & (Kalan et al. 2017) \\
\hline \multirow{2}{*}{$\begin{array}{l}\text { Passive } \\
\text { aircraft } \\
\text { transport }\end{array}$} & Aedes aegypti & The Netherlands & 2016 & No & No & $\begin{array}{l}\text { (Ibañez-Justicia et al. } \\
\text { 2017) }\end{array}$ \\
\hline & Aedes albopictus & The Netherlands & 2017 & No & No & (NVWA 2018) \\
\hline \multirow{8}{*}{$\begin{array}{l}\text { Passive } \\
\text { Sea/Ferry } \\
\text { traffic } \\
\text { transport }\end{array}$} & \multirow[t]{8}{*}{ Aedes albopictus } & Algeria & 2016 & Yes & Unknown & (Benallal et al. 2016) \\
\hline & & Italy (Sicilian islands) & 2017 & Yes & Yes & (Di Luca et al. 2017) \\
\hline & & $\begin{array}{l}\text { Italy (Tyrrhenian sea } \\
\text { islands) }\end{array}$ & 2017 & Yes & Yes & (Toma et al. 2017) \\
\hline & & Spain (Ibiza) & 2014 & Yes & Yes & (Barceló et al. 2015) \\
\hline & & Spain (Minorca) & 2015 & No yet & No yet & (Bengoa et al. 2016) \\
\hline & & Spain (Majorca) & 2013 & Yes & Yes & (Miquel et al. 2013) \\
\hline & & Greece & 2003 & Yes & Yes & $\begin{array}{l}\text { (Samanidou-Voyadjoglou } \\
\text { et al. 2005) }\end{array}$ \\
\hline & & Malta & 2009 & Yes & Check & (Buhagiar 2009) \\
\hline \multirow{3}{*}{$\begin{array}{l}\text { Natural } \\
\text { dispersal }\end{array}$} & \multirow[t]{3}{*}{ Aedes japonicus } & Italy (Austrian border) & 2015 & Yes & Yes & (Seidel et al. 2016b) \\
\hline & & Austria & 2011 & Yes & Yes & (Seidel et al. 2016a) \\
\hline & & Croatia & 2013 & Yes & Yes & (Klobucar et al. 2018) \\
\hline
\end{tabular}


Review pathways introduction invasive mosquito species

\begin{tabular}{|c|c|c|c|c|c|c|}
\hline Pathway & Species & Country (Region) & Year & $\begin{array}{l}\text { Later } \\
\text { establishment? }\end{array}$ & $\begin{array}{l}\text { Later } \\
\text { spread? }\end{array}$ & References \\
\hline \multirow[t]{15}{*}{ Unknown } & Aedes aegypti & Canary Islands (Spain) & 2017 & Unknown & Unknown & (ECDC 2018b) \\
\hline & \multirow[t]{5}{*}{ Aedes albopictus } & Spain (Catalonia) & 2004 & Yes & Yes & (Collantes et al. 2015) \\
\hline & & Spain (Valencia) & 2007 & Yes & Yes & (Roiz et al. 2007) \\
\hline & & Spain (Basque Country) & 2014 & No & No & (Delacour et al. 2015) \\
\hline & & The Netherlands & 2016 & No & No & (NVWA 2016) \\
\hline & & The Netherlands & 2017 & No & No & (NVWA 2018) \\
\hline & \multirow[t]{7}{*}{ Aedes koreicus } & Germany & 2015 & Unknown & Unknown & (Werner et al. 2016) \\
\hline & & Hungary & 2016 & Unknown & Unknown & (Kurucz et al. 2016) \\
\hline & & Belgium & 2008 & Yes & Yes & (Versteirt et al. 2012) \\
\hline & & Russia & 2013 & Unknown & Unknown & (Bezzhonova et al. 2014) \\
\hline & & Switzerland & 2015 & Yes & Yes & (Suter et al. 2015) \\
\hline & & Italy (Veneto) & 2011 & Yes & Unknown & (Capelli et al. 2011) \\
\hline & & Italy (Veneto) & 2012 & Yes & Yes & (Montarsi et al. 2013) \\
\hline & \multirow[t]{2}{*}{ Aedes japonicus } & The Netherlands & 2012 & Yes & Yes & $\begin{array}{l}\text { (Ibañez-Justicia et al. } \\
\text { 2014) }\end{array}$ \\
\hline & & Slovenia & 2013 & Yes & Yes & (Kalan et al. 2014) \\
\hline
\end{tabular}





\section{Chapter 5}

\section{Evaluating perceptions of risk in mosquito experts and identifying undocumented pathways for the introduction of invasive mosquito species into Europe}

Adolfo Ibáñez-Justicia ${ }^{1}$, P. M. (Marijn) Poortvliet ${ }^{2}$, Constantianus J.M. Koenraadt ${ }^{3}$

1. Centre for Monitoring of Vectors (CMV), Netherlands Food and Consumer Product Safety Authority (NVWA), Wageningen, The Netherlands

2. Department of Social Sciences - Strategic Communication, Wageningen University \& Research, Wageningen, The Netherlands

3. Laboratory of Entomology, Wageningen University \& Research, Wageningen, The Netherlands

Published in Medical and Veterinary Entomology (2018), doi: 10.1111/mve.12344 


\begin{abstract}
In several reported cases of the entry of invasive mosquito species (IMSs) into Europe, the introduction was associated with a specific pathway of introduction or dispersal. The identification of potential pathways for the introduction of IMSs and evaluations of the importance of the different pathways are key to designing proper surveillance strategies to promptly detect and control introductions in non-infested areas. The main goals of the present study were to identify other, previously undocumented, pathways of introduction into Europe, and to identify mosquito experts' perceptions regarding control m easures against IMS introductions via different documented pathways. At the European Mosquito Control Association (EMCA) conference in Montenegro in March 2017, a questionnaire was distributed among meeting participants to collect expert data. Results showed that ground transportation (by cars, trucks, etc.), passive natural dispersal and the shipping of used tyres are perceived as the most likely pathways. Introduction via aircraft did not appear to be well known and was not perceived as probable. This study shows that there were no pathways unknown to European experts that could lead to cryptic introductions into the experts' countries. Furthermore, the findings demonstrated that the perceived efficacy of surveillance and control is key to overcoming the constraints experienced and to supporting the implementation of actions against introductions.
\end{abstract}

Keywords: Aedes, action tendencies, EMCA, mosquito control, mosquito surveillance, questionnaire 


\section{Introduction}

Over the last decades, Europe has witnessed several introductions of exotic mosquito species with invasive potential, here term ed 'invasive m osquito species' (IMSs). Exam ples include the entry of the Asian tiger mosquito [Aedes albopictus (Skuse) (Diptera: Culicidae)] into Italy (Sabatini et al., 1990), the Am erican rockpool m osquito [ledes atropalpus (Coquillett)] and the yellow fever mosquito [Aedes aegypti (L.)] into the Netherlands (Scholte et al., 2009; Brown et al., 2011), the Asian bush mosquito [Aedes japonicus (Theobald)] into Switzerland, Germ any, Belgium and the Netherlands (Schaffner et al., 2009; Versteirt et al., 2009; Ibañez Justicia et al., 2014), and Aedes koreicus (Edwards) into Belgium, Italy, European Russia, the Swiss-Italian border, Hungary and Germ any (Capelli et al., 2011; Versteirt et al., 2012; Bezzhonova et al., 2014; Suter et al., 2015; Kurucz et al., 2016; Werner et al., 2016). The introduction and possible establishment of these IMSs represent a risk to public health due to the ability of some IMSs to transmit vector-borne diseases. Recently, established populations of Ae. albopictus have been involved in outbreaks of dengue and Chikungunya in southern France (Gould et al., 2010; La Ruche et al., 2010; Calba et al., 2017), and Chikungunya outbreaks in Italy (Rezza et al., 2007; Venturi et al., 2017), and established populations of Ae. aegypti caused an outbreak of dengue on Madeira island (Portugal) (Sousa et al., 2012). It has also been speculated that countries in which Ae. aegypti or Ae. albopictus mosquitoes are present may represent sites of future Zika virus outbreaks (Jupille et al., 2016).

Increasing international trade and international travel are the main drivers of the expansions in the native distributions of several mosquito vector species (Enserink, 2010). The first sighting of Ae. albopictus in Europe occurred in Albania and probably resulted from the trade of goods with China (Adhami \& Reiter, 1998). However, only when the species was introduced into Italy in 1990 through the import of used airplane tyres from Atlanta (GA, U.S.A.) (Dalla Pozza \& Majori, 1992), and following its subsequent spread in Italy, was it considered a threat to public health (Knudsen et al., 1996).

In several of these events, the introduction was clearly associated with a specific pathway. Trade in used tyres has been incriminated in the introduction of Ae. albopictus into European countries such as France, Belgium, the Netherlands, Croatia, Montenegro and Spain (Dalla Pozza \& Majori, 1992; Schaffner \& Karch, 2000; Petrić et al., 2001; Scholte \& Schaffner, 2007; Generalitat de Catalunya, 2008; Scholte et al., 2010), as well as Ae. japonicus into Belgium (Versteirt et al., 2009), and Ae. atropalpus and Ae. aegypti into the Netherlands 
(Scholte et al., 2010). Trade in ornam ental lucky bam boo plants has also been linked to the introduction of Ae. albopictus into the Netherlands and Belgium (Scholte et al., 2008; Dem eulem eester etal., 2014). Ground transport from areas heavily infested with $A e$. albopictus resulted in the spread of this species from Italy into new areas in Europe such as southern France, Germany, the Balkans, the Czech Republic, Spain and Switzerland (Aranda et al., 2006; Scholte \& Schaffner, 2007; Sebesta et al., 2012; Kam pen et al., 2013).

Identifying all the potential pathways for the introduction of IMSs at the European level and evaluating the respective importance of these different pathways is key to designing a surveillance strategy that will promptly detect and control introductions of IMSs in noninfested areas.

Surveillance and control measures against IMSs are not likely to be implemented if, for instance, experts experience little interest from government organizations. In this event, their belief that taking action will be effective will be reduced. In order to obtain relevant information, it is important to collect the opinions and perceptions of country experts in mosquito surveillance and control. For this purpose, the present study used protection motivation theory (PMT) (Boer \& Seydel, 1996), which proposes that the intention to protect oneself (action tendency) depends upon four factors: (a) the perceived severity of a given hazard; (b) the perceived probability of its occurrence, or vulnerability to this hazard; (c) the perceived effectiveness of the preventive behaviour recommended to deal with the hazard (response efficacy), and (d) the individual's level of confidence in his or her ability to undertake the recommended preventive behaviour against the hazard (self-efficacy) (Boer \& Seydel, 1996). In addition to these four factors, a fifth factor can be included: the perception of constraints that impede the implementation of preventive behaviour. Perceived constraints are often considered as important predictors of health promotion behaviour (Janz \& Becker, 1984) and can be expected to play a role in explaining the action tendencies of the experts consulted to support the implementation measures that would prevent the introduction of IMSs. In other words, even if an expert perceives the introduction of an IMS as a threat, and believes that surveillance and control actions will effectively reduce the threat, perceived constraints may prevent that individual from supporting the implementation of actual actions against the IMS. As well as playing a role in health promotion and disease prevention, PMT has been successfully used to predict people's actions to prevent injury, share in environm ental concerns, and to im prove public safety (Floyd et al., 2000; Westcott et al., 2017). Protection motivation theory can be used to influence and predict various behaviours 
(Boer \& Seydel, 1996) and, if appropriately used in the IMS context, could influence attitudes towards supporting the taking of action against the introduction of IMSs to prevent their establishment.

The main goals of the current study were to identify other, previously undocumented, pathways of introduction and dispersal of IMSs in Europe, and to identify experts' perceptions regarding control measures against IMS introductions via different documented pathways. In addition, as well as providing descriptive information on perceptions of the risk for introductions, PMT provides insight into what drives experts' attitudes towards supportive actions against the introduction of IMSs.

\section{Materials and methods}

\section{Questionnaire}

Data were collected by distributing a questionnaire among experts during the European Mosquito Control Association (EMCA) conference in Bečići, Montenegro (March 2017). The main objectives of the study and instructions for the completion of the questionnaire were introduced during the plenary opening of the conference by the president of the EMCA and by the first author (AI-J). Subsequently, the questionnaire was distributed to all participants. To ensure independent answers, participants were asked to answer all questions without consulting other participants. Completed questionnaires were collected up to the end of the conference. Participants who arrived after the opening of the conference were asked to participate in the study during the openings of conference sessions over the next days.

Introduction pathways identified from the literature were categorized in four main groups: trade in used tyres; import of lucky bamboo plants; passive transport in vehicles (by road, aeroplanes, ships), and natural dispersal. The questionnaire was designed following the methodology of PMT (Boer \& Seydel, 1996) taking into account the following factors: (a) the perceived severity of a threatening event; (b) the perceived probability of the occurrence, or vulnerability, and (c) the efficacy of the recommended preventive behaviour (response efficacy). Self-efficacy (the fourth factor in PMT) was omitted in the present study because this factor measures the individual's level of confidence in his or her own ability to undertake the recommended preventive behaviour; it is considered less relevant to the present study because IMS control measures take place at a collective rather than an individual level. 
Instead, an additional factor that assessed perceived constraints with respect to the implementation of preventive behaviour was included. This was done because there are many practical issues that may limit the likelihood of an IMS control programme being successful. This factor was expected to play a role in explaining the action tendencies of participants in supporting the implementation of IMS surveillance and control measures.

The questionnaire was divided into several sections. The first section asked for general information about the participant, including his or her name and institution (not obligatory), gender, age, country and region. Nine subsequent questions addressed the knowledge of the participant regarding pathways for the introduction of IMSs. Questions could be answered with any of the responses 'Yes', 'No' and 'Don't know'. Correct answers were awarded 1 point. Incorrect or blank answers scored no points. Thus, a final knowledge index of 0-9 points (on a 10-point scale) was computed. Thirdly, respondents were asked to evaluate four statements on the perceived severity of introductions of IMSs into their respective countries/regions (e.g. 'Introduction of Aedes albopictus in my country/region can lead to irreversible establishm ent and dispersal of this vector species'). Statem ents were evaluated using a Likert-based scale with five response alternatives $(1=$ do not agree at all; $2=$ do not agree; $3=$ neither agree nor disagree; $4=$ agree; $5=$ fully agree). Next, respondents were asked to evaluate six statements on the perceived vulnerability of their respective countries/regions to the introduction of IMSs via different known pathways (e.g. 'The chance of introduction of Aedes invasive species in my country/region with import of used tyres is rather large'). Statem ents were evaluated using a 5-point Likert scale similar to that used in the evaluation of severity. Then, respondents were asked to evaluate four statements on the perceived response efficacy of measures for the surveillance and control of IMS introductions into their respective countries/regions (e.g. 'Invasive Aedes species surveillance and control measures implemented in my country/region lead to accurate evaluation of potential introduction locations for Aedes species'). Statem ents were evaluated using a 5-point Likertbased scale similar to that used to evaluate severity. After the response efficacy items, respondents were asked to evaluate four statements on perceived constraints with respect to the im plem entation of IMS surveillance in their respective countries/regions (e.g. 'In $\mathrm{m} \mathrm{y}$ country/region, adequate surveillance of invasive Aedes species will be difficult to implement because of the lack of interest of the governm ental organizations'). Statem ents were evaluated using a 5-point Likert scale similar to that used for severity. With the aim of collecting more information about the constraints faced by experts in implementing IMS 
surveillance, participants were asked to write these constraints in this section and to assign a score to each of the constraints experienced. Lastly, participants were asked to respond to six statements evaluating action tendencies regarding the implementation of IMS surveillance and control $\mathrm{m}$ easures in their respective countries/regions (e.g. 'To what extent do you agree that IMS surveillance needs to be implemented in all countries/regions of Europe?'). These statements were also answered using a 5-point Likert scale.

After the questions and statements on knowledge, perceived severity, perceived vulnerability, perceived response efficacy, perceived constraints and action tendencies, the questionnaire also asked whether participants were aware of other pathways of introduction that had not been reported previously in the literature. Participants were asked to indicate at least one other pathway of introduction in their respective countries/regions. These data are important for detecting new or neglected pathways for the introduction of IMSs in Europe.

The questionnaire is included in the supplementary data (Appendix S1). Correct answers to the knowledge questions are provided in the questionnaire using bold text. Scores across participants on individual questions are provided in Appendix S2.

\section{Data analysis}

The internal consistency of the set of items representing a variable was checked and these items were collapsed into a single index after the Cronbach's alpha values (Cronbach, 1951) were verified as being 0.7 or higher. Alpha values were computed for each variable measured in the questionnaire: Knowledge; Severity; Vulnerability; Response efficacy; Constraints, and Action tendencies. This test is used when a tool includes multiple questions or statements that have scaled answers, and when there is a need to determine whether the scale is reliable. Pearson product-moment correlation coefficients were computed and linear regression analyses were conducted to identify relationships among the variables. Variables included in the regression m odel were those for which item $\mathrm{s}$ form ed a reliable scale (Cronbach's $\alpha>0.7$ ), which allowed the results to be combined into one index, and also variables that were significantly correlated with the dependent variable in the Pearson correlation coefficient analysis. Finally, a mediation analysis was performed to explore whether a relationship between an independent variable and a dependent variable runs via a mediating variable (Baron \& Kenny, 1986). This means that an independent variable (e.g. perceived constraints) influences a mediating variable (the mediator; e.g. perceived response efficacy), which, in 
turn, influences the dependent variable (e.g. action tendencies). Therefore, the mediator provides insight into the underlying process of the relationship between the independent and the dependent variable. To formally test for mediation, a bootstrap analysis (Shrout \& Bolger, 2002; Preacher \& Hayes, 2004) was employed to test the reduction in the direct effect. This approach involves computing 95\% confidence intervals (CIs) (5000 bootstrap resamples) around indirect effects; mediation is indicated by CIs that do not include 0. All statistical analyses were performed using spss Statistics for Windows Version 17.0 (SPSS, Inc., Chicago, IL, U.S.A.).

\section{Results}

\section{Demographic characteristics}

In total, 118 participants from 25 countries participated in the EMCA conference in 2017. The countries with the largest delegations were Germany and Greece with 18 (15.3\%) and 15 (12.7\%) participants, respectively. There were also participants from the non-European countries of the U.S.A., Israel, Thailand and Iran (7/118, 5.9\%). Forty-eight participants from 15 European countries filled out the questionnaire. These respondents came from Belgium, Croatia, the Czech Republic, France, Germany, Greece, Italy, Montenegro, the Netherlands, Portugal, Romania, Serbia, Spain, Sweden and Switzerland (Fig. 1). Of these 48 participants, 31 provided information on the name of their organization in the questionnaire. Fifteen participants worked for surveillance agencies, seven for universities, five for control agencies, and four for public health authorities.

As at March 2017, of the 15 European countries represented in responses to the questionnaire, all except Sweden and Portugal had previously reported occurrences of $A e$. albopictus. These countries were Belgium (not established to date), Croatia, the Czech Republic (not established to date), France (including Corsica), Germany, Greece, Italy (including Sardinia, Sicily, Lampedusa and other islands), Montenegro, the Netherlands (not established to date), Romania, Serbia (not established to date), Spain and Switzerland. Except for records of occurrence on the island of Madeira (Portugal), introductions of Ae. aegypti had been reported only in the Netherlands, but the species had not established. Also in the Netherlands and in Italy, Ae. atropalpus introductions had been reported without further establishment. Six of the countries represented by respondents to the questionnaire had reported occurrences of Ae. japonicus; these included Belgium, Croatia, France, Germany, 
the Netherlands and Switzerland. Finally, Ae. koreicus had been reported in four countries, including Belgium, Germany, Italy and Switzerland.

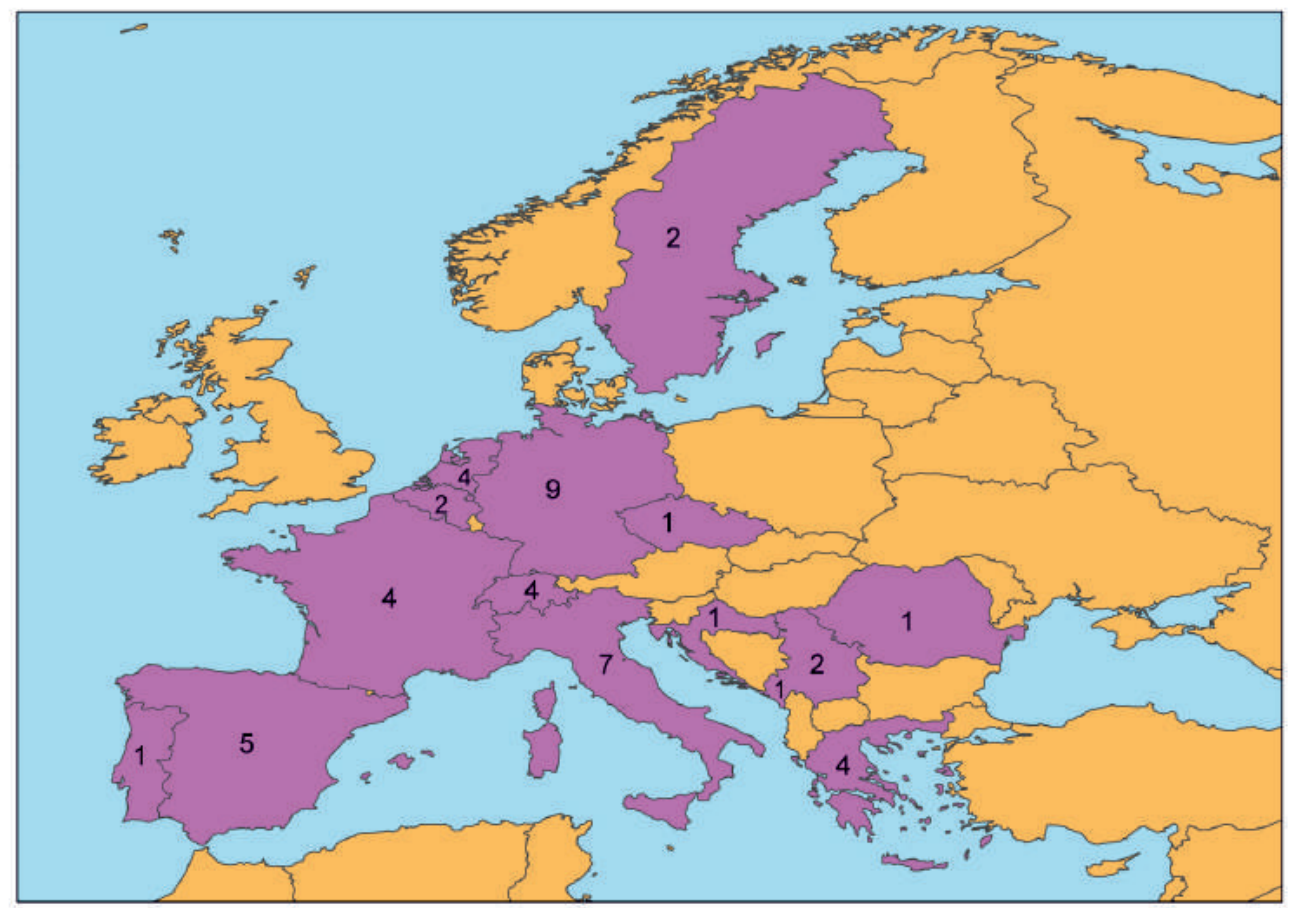

Fig. 1. Countries (in purple) and respective numbers of conference participants completing the study questionnaire.

Of the 48 participants, 33 were male and 14 were female; one participant did not report gender. The average age of respondents was 40.2 years in wom en (range: 26-53 years), and 44.3 years in m en (range: 27-65 years).

\section{Questionnaire results}

\section{Knowledge}

On a scale of 0-9 points (10-point scale), the m ean \pm standard deviation (SD) score of all participants was $6.33 \pm 1.79$. Figure 2 shows total knowledge scores across the 48 participants. Eight participants (16.7\%) answered all nine questions correctly. 


\section{Knowledge score participants}

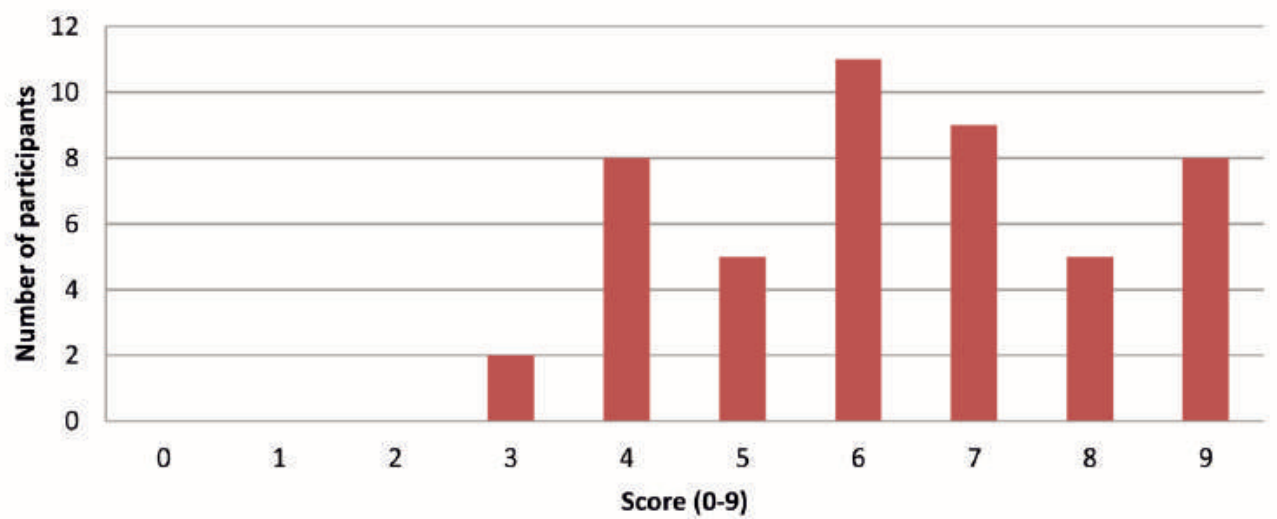

Fig. 2. Knowledge scores of conference participants who completed the study questionnaire.

Knowledge questions evaluation

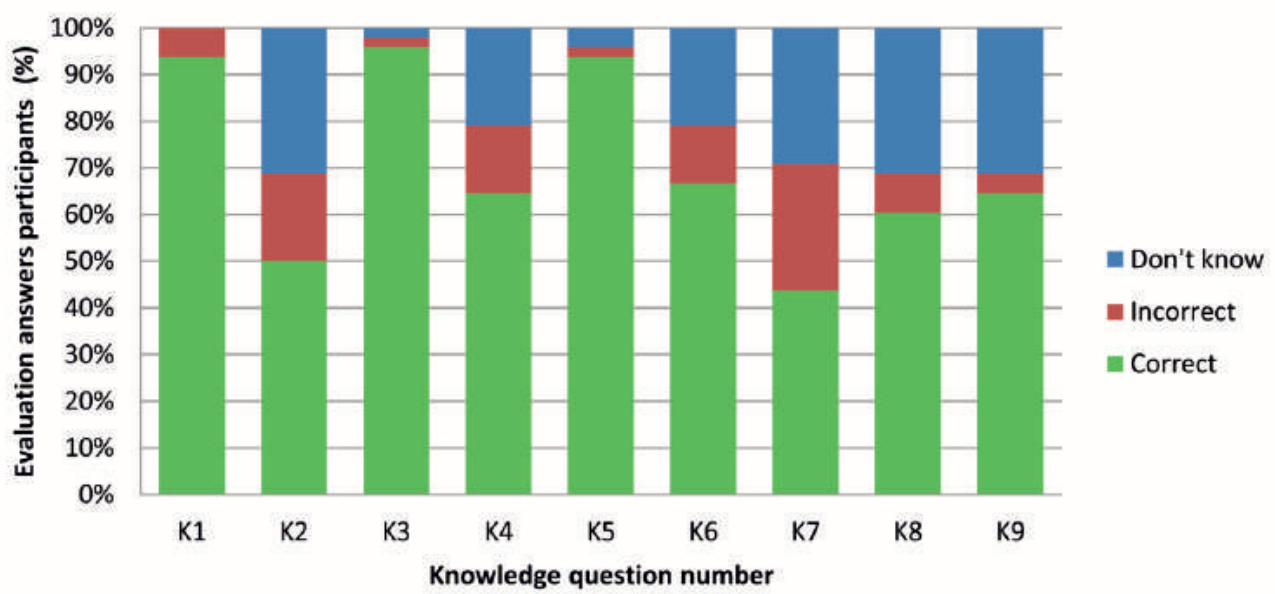

Fig. 3. Evaluation of answers to the nine knowledge-based items on the questionnaire.

Figure 3 evaluates responses to each of the nine knowledge questions across all 48 participants. In general, participants had good knowledge of the significance of the import of used tyres (question K1; Fig. 3), the inability of Ae. aegypti eggs to survive cold winters (K3), and the association of Ae. albopictus with passive transport by road traffic (K5). Overall, $19.0 \%$ of all knowledge questions were answered with the 'Don't know' option. Some questions appeared more difficult for the participants because around $30 \%$ of responses 
to these questions used the 'Don't know' option. Overall, 30.0\% of participants had difficulty in correctly identifying the only implication (to date) of the introduction of one IMS species (Ae. albopictus) through the import of lucky bamboo plants (K2 and K8). Similarly, participants also showed a lack of knowledge of the absence of Ae. albopictus populations in Denmark (K9). Answers to question K7 (absence of evidence of the passive transport of Ae. albopictus inside aircraft) showed high rates of both incorrect answers (27.0\%) and 'Don't know' options $(29.0 \%)$.

\section{Severity}

The variation in severity responses is presented in Fig. 4. The internal consistency of the scale was low (Cronbach's $\alpha=0.39$ ). Therefore, results were not com bined into one index. Nonetheless, the median of all severity scores is 5, the maximum score on the scale, indicating agreement on the perceived severity of introductions of IMSs. Introductions of IMSs were perceived to lead to the establishment and dispersal of vector IMSs, to increase the probability of the transmission of vector-borne diseases, and to heighten the risks to human and veterinary health.

\section{Vulnerability}

The variation in vulnerability responses is presented in Fig. 4. Similarly to the severity scores, the internal consistency of the scale was relatively low (Cronbach's $\alpha=0.54$ ), suggesting that the five pathways of introduction presented were perceived differently in terms of vulnerability. Therefore, the results were not combined into one index. The median scores for vulnerability to the pathways varied from 3 to 4 . The median of all vulnerability scores was 4. Participants perceived high vulnerability (median Likert scale score: 4) to passive ground transport, natural dispersal from neighbouring countries and import through used tyre shipments. Vulnerability to passive transport inside aircraft, with lucky bamboo plants or via maritime ferry traffic was not perceived as particularly high or low (median Likert scale score: 3 ). 


\section{Response-efficacy}

The variation in answers to items on response efficacy is presented in Fig. 4. The four items form ed a reliable scale (Cronbach's $\alpha=0.78$ ), which allowed the results to be com bined into one index. In general, there was a high perception of the efficacy of case surveillance and control with reference to the 'evaluation of potential introduction locations' and 'early detection of IMS', as well as for 'certainty about IMS presence' (m edian Likert scale scores: 4). By contrast, participants expressed a much lower perception of the response efficacy of surveillance and control in their countries with reference to the elimination of newly detected IMS foci (median scores: 3).

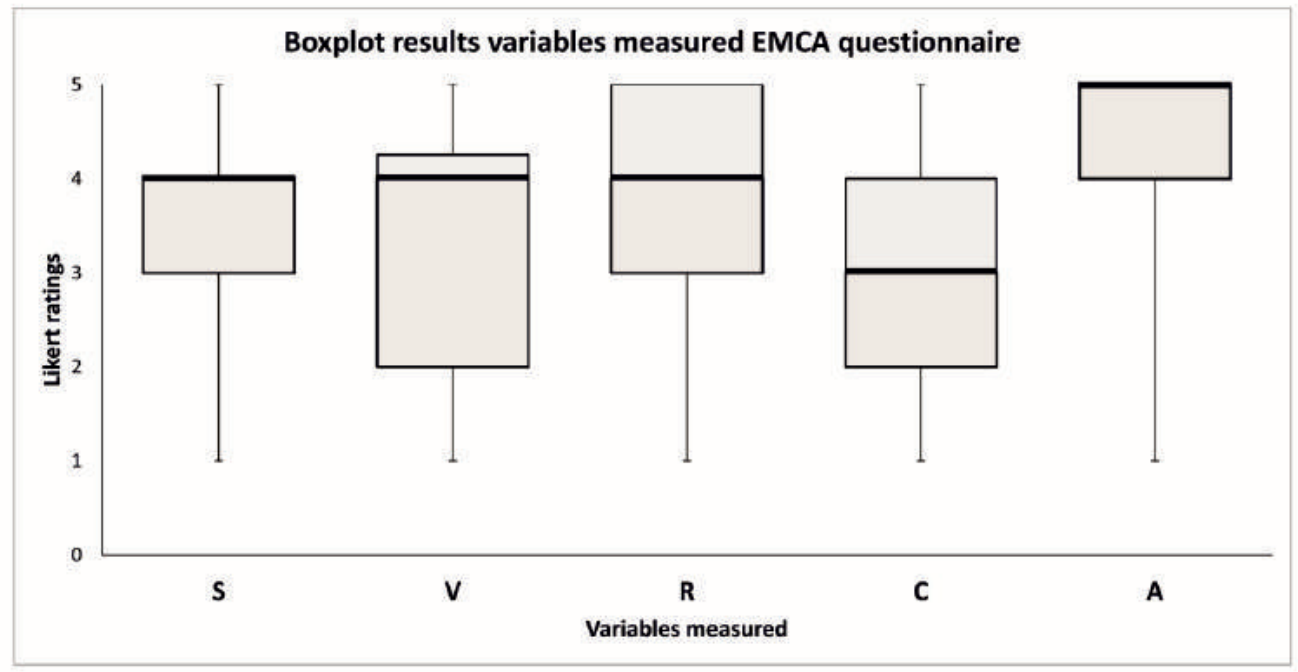

Fig. 4. Likert scale-based ratings of variables measured in the questionnaire. The boxplot represents scores on the different questions per variable measured. The boxes represent the lower and upper quartiles of the values recorded in the questionnaire. The bold horizontal line represents the median of the values. Lower and upper whiskers represent minimum and maximum values, respectively. S, severity; V, vulnerability; R, response efficacy; C, constraints; A, action tendencies.

\section{Constraints}

The variation in the constraints responses is presented in Fig. 4. The four items formed a reliable scale (Cronbach's $\alpha=0.80$ ), which allowed the results to be com bined into a single index. The median score for all constraints answers was 3, a neutral value for participants' perceptions of constraints against the implementation of IMS surveillance in their respective countries. The constraint 'lack of budget' gained higher m edian values am ong participants, whereas 'lack of vector surveillance team s' was considered a less im portant constraint. Other 
constraints optionally added by participants in their responses to the questionnaire were: lack of coordination among institutions, regional authorities and ministries; lack of unrestricted access to breeding sites; lack of control capacities; lack of universal concern; lack of awareness of local authorities about the importance of source reduction in mosquito control; lack of perception of risk, and lack of communication. The scores for these other perceived constraints were not analysed further in view of the diversity of these data.

\section{Action tendencies}

The variation in action tendency responses is presented in Fig. 4. The six items formed a reliable scale (Cronbach's $\alpha=0.70$ ), which allowed the separate item s to be collapsed into a single index. In general, there was a strong positive attitude towards the implementing of IMS surveillance and control in Europe, and participants agreed on the necessity of investing funds and efforts, including European legislation (median Likert scale score: 5). Participants also tended to agree on the necessity to eradicate Aedes IMS in Europe (median Likert scale score: 4).

\section{Other introduction pathways}

Participants reported the following other pathways of introduction: (a) import of plants other than lucky bamboo; (b) import of agricultural products; (c) import of recycled products other than used tyres; (d) import of ornaments [e.g. stone containers (fountains)]; (e) container shipping (inside international freight containers destined for merchant markets); (f) unintended introduction by people using cars, caravans, camper vans, etc.; (g) truck transport by train [e.g. the Rollende Landstrasse (RoLa) in Germany]; (h) natural dispersal (e.g. gradual shifts in the areas of Europe in which exotic Aedes spp. can live as a result of climate change); (i) travel by entomologists, and (j) introduction by refugees.

Several pathways of introduction mentioned by participants had already been reported in the literature and relate to passive ground transportation of IMSs (e.g. inside freight containers, private vehicles, trucks and trains) or to the natural dispersal of IMSs. Interesting alternative pathways relate to the import of products that have not been reported yet, such as plants other than lucky bamboo, agricultural and recycling products, and outdoor ornaments (e.g. stone fountains). Unexpected pathways perceived by some participants as potentially playing a role 
in the introduction of Aedes IMSs were travel by entomologists and the migration of refugees into European countries.

\section{Correlation analysis}

Pearson product-moment correlation coefficients were calculated to identify relationships among the computed variables for which Cronbach's alpha results indicated a reliable scale (action tendencies, response efficacy and constraints) (Table 1). A medium positive correlation $(P<0.05)$ was observed between action tendencies with regard to support of the implementation of IMS surveillance and control measures and perceived response efficacy of the surveillance and control of IMS introductions. In other words, if participants felt that surveillance and control programmes were efficacious in their own countries, they tended to support such programmes. The results also show that there is a significant negative correlation $(P<0.01)$ between perceived response efficacy of surveillance and control measures for IMS introductions, and perceived constraints against the implementation of IMS surveillance. In other words, when few constraints are perceived with respect to the implementation of IMS surveillance, participants perceive surveillance and control as more effective.

Table 1. Means, standard deviations (SDs), and Pearson correlations among variables measured on the questionnaire administered during the European Mosquito Control Association (EMCA) conference. Significant correlations $(P<0.05)$ are indicated in bold.

\begin{tabular}{lcccc}
\hline \hline & Mean & SD & Response efficacy & Constraints \\
\hline Response efficacy & 3.59 & 0.88 & & \\
Constraints & 3.18 & 0.94 & $\mathbf{- 0 . 5 6 5}$ & \\
Action tendencies & 4.36 & 0.49 & $\mathbf{0 . 3 2 3}$ & 0.075 \\
\hline
\end{tabular}

\section{Linear regression analysis}

Linear regression was used to examine the effects of predictor variables perceived Response efficacy and perceived Constraints, on the outcome variable Action tendencies. This analysis sought to examine if these two independent variables explain the participants' support for the implementation of IMS surveillance and control measures. Perceived Constraints was included in the model (no correlation with Action tendencies) based on the hypothesis that 
the more constraints perceived, the less experts will be motivated to support the implementation of surveillance and control. On the contrary, the more surveillance and control are perceived as effective, the more experts will tend to support the implementation of these actions.

Table 2. Results of the multiple regression analysis of factors affecting the action tendencies of mosquito experts towards supporting the implementation of surveillance and control measures against invasive mosquito species. CI, confidence interval. Standardized regression coefficients are reported $* P<0.05, \dagger P<0.01$

\begin{tabular}{llll}
\hline \hline Variables & $\boldsymbol{\beta}$ & $\mathbf{9 5 \%} \mathbf{C I}$ & t-statistic \\
\hline Constraints & $0.370^{*}$ & $0.018-0.362$ & 2.227 \\
Response-efficacy & $0.530^{\dagger}$ & $0.106-0.473$ & 3.186 \\
\hline
\end{tabular}

The results of the multiple linear regression analysis (Table 2) show that both the perceived response efficacy of surveillance and control measures, as well as the perceived constraints with respect to the implementation of IMS surveillance, significantly affect action tendency in this multivariate regression model. This confirms one of the study's initial hypotheses, which assumed that experts tend to support actions against introductions of IMSs when they perceive IMS surveillance and control as effective against the introductions. By contrast with the absence of a significant correlation in the Pearson product-moment analysis, the perception of constraints against the implementation of these control measures positively affected the tendency to support actions against introductions $(P<0.05)$.

\section{Mediation analysis}

The previous results show that no significant correlation was found between the values of perceived constraints and action tendencies $(P>0.05)$. However, there was a negative correlation between perceived constraints and perceived response efficacy $(P<0.01)$, and a positive correlation between perceived response efficacy and action tendencies $(P<0.05)$, pointing to a possible indirect path from perceived constraints to action tendencies via response efficacy. To test for this, a mediation analysis was performed. The results gave a $95 \% \mathrm{CI}$ of -0.2916 to -0.0343 . Based on this result ( 0 is not included in the $95 \% \mathrm{CI}$ ), the present authors conclude that the mediated effect is indeed significantly different from 0 $(P<0.05$; indirect effect: $-0.15, \mathrm{SE}=0.07)$, which $\mathrm{m}$ eans that perceived constraints 
influence action tendencies via perceived response efficacy (Fig. 5). In other words, the perceived efficacy of surveillance and control is key to the European experts overcoming the perceived constraints and supporting actions to implement the surveillance and control of IMSs.

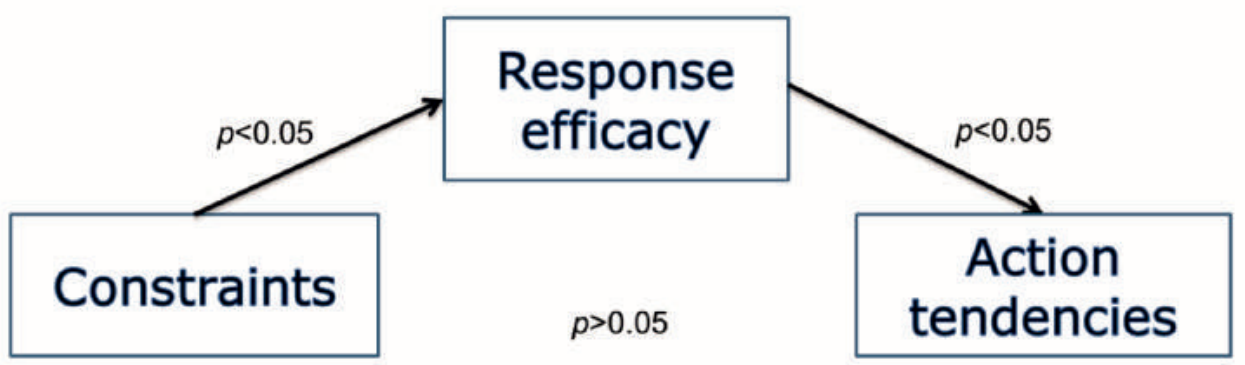

Fig. 5. Representation of mediation analysis results for the measured variables Constraints, Response efficacy and Action tendencies.

\section{Discussion}

This study was designed to investigate if other, previously undocumented, pathways for the introduction of Aedes IMSs are present in Europe, to identify experts' perceptions of the risk associated with the different documented pathways, and to investigate what drives experts' attitudes towards supporting actions to prevent and control introductions. The investigation showed that the main pathways have been adequately documented in the literature, and that no other pathways are hidden. Specifically, passive ground transportation (in cars, trucks, etc.) is perceived by experts as the most likely pathway of introduction. The investigation also highlighted the perceived vulnerability to introductions through the natural dispersal of Aedes IMSs and through shipments of used tyres within Europe. Introduction via aircraft was not perceived as probable by the participants. Other potential pathways indicated referred to the import of plants (other than lucky bamboo), import of agricultural and recycling products, and import of outdoor ornaments. Finally, the study also demonstrated that the action tendencies of mosquito experts for implementing surveillance and control of IMSs are affected by their perceptions of the efficacy of these programmes and their perceptions of the constraints they encounter.

As the questionnaire was administered during a conference of the EMCA, only a small percentage of participants were public health officers. Most of the participants at the conference who completed the questionnaire were involved in the surveillance and control of 
mosquitoes, and were often not the final decision makers on issues related to vectors and public health in their countries. In this respect, the measured action tendencies of the experts consulted could be considered as less relevant in view of the profile of the participants. However, their support, advice and reports and the results achieved by implementing surveillance and control measures are key factors on which policymakers can base decisions at regional or national levels, including those on whether surveillance or control are necessary, and if they can be financed and implemented.

In general, if participants feel that surveillance and control programmes are effective, they tend to support them and to experience fewer constraints with respect to the implementation of these measures. A higher perception of the efficacy of surveillance and control measures against IMS introductions was associated with a stronger inclination to support such actions. Furthermore, the perceived efficacy of surveillance and control was negatively associated with participants' perceptions of constraints against the implementation of these measures.

The questionnaire was designed in such a way that all relevant pathways were mentioned repeatedly, allowing the participant to think about the risk for pathways of IMS introduction. The present authors consider that if the participant had knowledge of other relevant pathways in his or her country, he or she would have recorded this at the end of the questionnaire. Of the 'other' pathways of introduction m entioned by participants, several have been suspected to introduce IMSs into or across Europe (e.g. natural dispersal, passive ground transport). Other pathways mentioned need to be evaluated for their contributions to introductions because, to date, they have not been implicated in the introduction or movement of IMSs. For example, import of plants or plant material was pointed out as a possible pathway. In 2017, Ae. albopictus was found inside a flower auction house in the Netherlands [Nederlandse Voedsel- en Warenautoriteit (NVWA), 2017]. Transport and trade of lawn ornaments, plant pots and a wide variety of stone or concrete basins may serve as means of spreading eggs and larvae of Ae. japonicus in the U.S.A. (Kaufman \& Fonseca, 2014). To date, this pathway has only been suspected as the route of introduction to the French Riviera of Ae. albopictus from China in stone fountains (ECDC, 2012). An unexpected potential pathway mentioned in the questionnaire was the migration of refugees to European countries. The present group believes that as the only possible way for this route of introduction to succeed depends on the presence of eggs in a refugee's belongings, the introduction of an IMS via this pathway is very unlikely. Nonetheless, the introduction of mosquito-borne diseases (e.g. malaria) has 
been associated with the $\mathrm{m}$ igration of refugees (Andriopoulos et al., 2013), and this $\mathrm{m}$ ay be why the respective participant indicated this pathway.

Among the documented pathways, passive ground transport (in cars, trucks, caravans, trains, etc.) was perceived as the most important mode of introduction of Aedes IMSs in participants' countries. This is indeed an important pathway of introduction, and also of dispersal across highly infested regions for Ae. albopictus in southern Europe and neighbouring regions. In this way, this species has colonized southern France from Italy and is extending its range northwards via the main highways. In 2015 it was detected in Strasbourg (France) (B. Mathieu, personal communication, 2018). In Switzerland, it became evident that the European m otorway E35 is a key route of invasion into northern Europe (Flacio et al., 2016). Findings at service stations along various main motorways in southern Germany also indicated repeated introductions of Ae. albopictus into Germ any (Becker et al, 2013). Unintended movements of IMSs by private ground vehicles have been confirmed in Catalonia (Spain) after a study on m ain roads in 2016 (Eritja et al., 2017). In European regions that are further from established populations (e.g. Belgium, the Netherlands), introductions through private vehicles may be considered less likely because mosquitoes may escape the transporting vehicle during stops made before these countries are reached.

The natural dispersal of IMSs is also considered a probable mode of introduction of IMSs into the survey participants' countries. The adult flight range of Ae. albopictus is approxim ately $200 \mathrm{~m}$ (ECDC, 2012; Medlock et al., 2015), which im plies that adults of this species remain in the immediate surroundings of the breeding sites from which they emerged. Field studies in dengue-affected areas confirmed a wider dispersal range of adults of at least $800 \mathrm{~m}$ within a 6 day period (Honorio et al., 2003), m aking natural dispersal in European areas that are climatically suitable more likely. A species that demonstrates an example of the natural spread of a mosquito species in Western Europe is Ae. japonicus. This species exploits a wider diversity of potential breeding sites than Ae. albopictus (artificial and natural) (Medlock et al., 2015), and has expanded its distribution range from colonized areas in Europe.

Trade in used tyres has been confirmed as the route of many first introductions of IMSs into European countries, and remains a source of IMS movement within and between European countries. This pathway was well known to the respondent experts, although some participants did not agree on this potential pathway in their regions. This may be because this 
type of trade is of low importance in some countries or because of the way used tyres are stored (indoors or outdoors) at used-tyre facilities.

Most of the participants did not agree or disagree on the likelihood of the introduction of IMSs by aircraft. Com bined with the high percentage of incorrect and 'Don't know' answers in the questions evaluating knowledge on this pathway of introduction, this signals some level of uncertainty around this topic or may indicate that this pathway is not subjected to adequate surveillance in most of the countries represented in the study. In Europe, there is recent evidence of introductions of Ae. aegypti at Schiphol Airport (Ibañez-Justicia et al., 2017). Further, because of the increase in international travel and trade, air travel may be considered one of the most efficient methods of transporting IMSs over large distances as m osquitoes $\mathrm{m}$ ay follow their hum an hosts into aircraft unnoticed (Gratz et al., 2000).

Introductions of IMSs with lucky bamboo plants or via maritime ferry transport were perceived as less probable by the study participants. Nonetheless, both pathways have been important in introducing Ae. albopictus into European regions. For instance, since 2010 Ae. albopictus specimens have been found annually during monitoring at lucky bamboo importing companies in the Netherlands. One reason why the likelihood of introductions with lucky bamboo plants was perceived as low may refer to the low volume of this product imported (or even no import of this product at all) into the various participants' countries. The present authors do not know the volumes of lucky bamboo plants imported into countries in Europe. Similarly, participants from countries such as Germany and Belgium, without shortdistance maritime ferry transport to regions with established IMS populations in the Mediterranean Sea, may perceive the risk for introductions through their harbours via this pathway as lower. Maritime ferry transport has been implicated as the most likely route of introductions of Ae. albopictus into Sicily and the Tyrrhenian islands from mainland Italian harbours (Di Luca et al., 2017; Tom a et al., 2017).

The observed relationship between the perceived effectiveness of surveillance and control measures, perceptions of constraints to the implementing of these measures, and the action tendencies of experts is relevant for their implementation in Europe. Regression analyses demonstrated that the perceived efficacy of the surveillance and control of IMSs is key to experts overcoming such constraints and supporting the implementation of actions against introductions of IMSs. As the results show with relation to perceived severity, the introduction, establishment and spread of IMSs in Europe are considered by experts to cause severe negative consequences, and the implementation of surveillance and control is seen by 
the mosquito experts consulted as effective against these introductions. One important recommendation to promote IMS surveillance and control measures is to increase the perceived effectiveness of the surveillance and control of IMSs by promoting and increasing the visibility of these activities. Investments in risk-based surveillance, research into new effective techniques of IMS detection and control, and the publication of IMS research in specialist journals will engender the support of actions against undesired IMS introductions in Europe.

Among the principal constraints, lack of budget is the most common, but other relevant constraints were lack of coordination and communication among institutions, and difficulty in gaining access to breeding sites for control purposes. In contexts in which an Aedes IMS has been recently detected, the combination of these constraints may threaten the effective implementation of control measures. Furthermore, mosquito surveillance is perceived as important to the collection of information about the presence of Aedes IMSs, but not in terms of supporting the elimination of newly detected foci at an early stage.

The climatological and geographical differences among countries may explain the variability in answers obtained on the perceived severity of introductions of IMSs. For example, the seriousness of the establishment of IMSs and the probability of transmission of associated vector-borne diseases were perceived as low in countries such as Sweden. In general, answers to these questions indicated the opinions and concerns of the experts about the problem of introductions of IMSs.

The present study shows that there are no pathways of introduction of Aedes IMSs unknown to experts, which may lead to cryptic introductions into the experts' countries. Passive ground transport was perceived as the most important pathway for introducing IMSs across European regions, and introduction via aircraft was not perceived as probable by the participants, which raises the question of whether this pathway is subject to adequate surveillance in European regions. Additionally, the removal of apparent constraints against the implementation of surveillance and control programmes, and the resulting high levels of perceived efficacy of surveillance and control programmes are key to enabling European mosquito experts to support the implementation of actions against IMSs. Furthermore, the present group strongly argues that the constraints perceived by experts must be considered and preferably diminished because perceptions of constraints lower levels of response efficacy, which, in turn, lower the tendencies of experts to support the implementation of actions against IMSs. 
Finally, the present authors recommend that the pathways and origins of new IMS findings be investigated and reported in journals that specialize in reporting on mosquito surveillance.

\section{Acknowledgements}

The authors acknowledge their European Mosquito Control Association (EMCA) colleagues Igor Pajovic, Spiros Mourelatos and Jan Lündstrom for allowing, announcing and supporting the study during the conference in Montenegro in March 2017. The authors especially acknowledge the EMCA participants at the conference for filling out the questionnaire. 


\section{Supporting information}

S1. Questionnaire on the perception of risk for the introduction of invasive Aedes species distributed among meeting participants at the European Mosquito Control Association (EMCA) conference, March 2017, Montenegro.

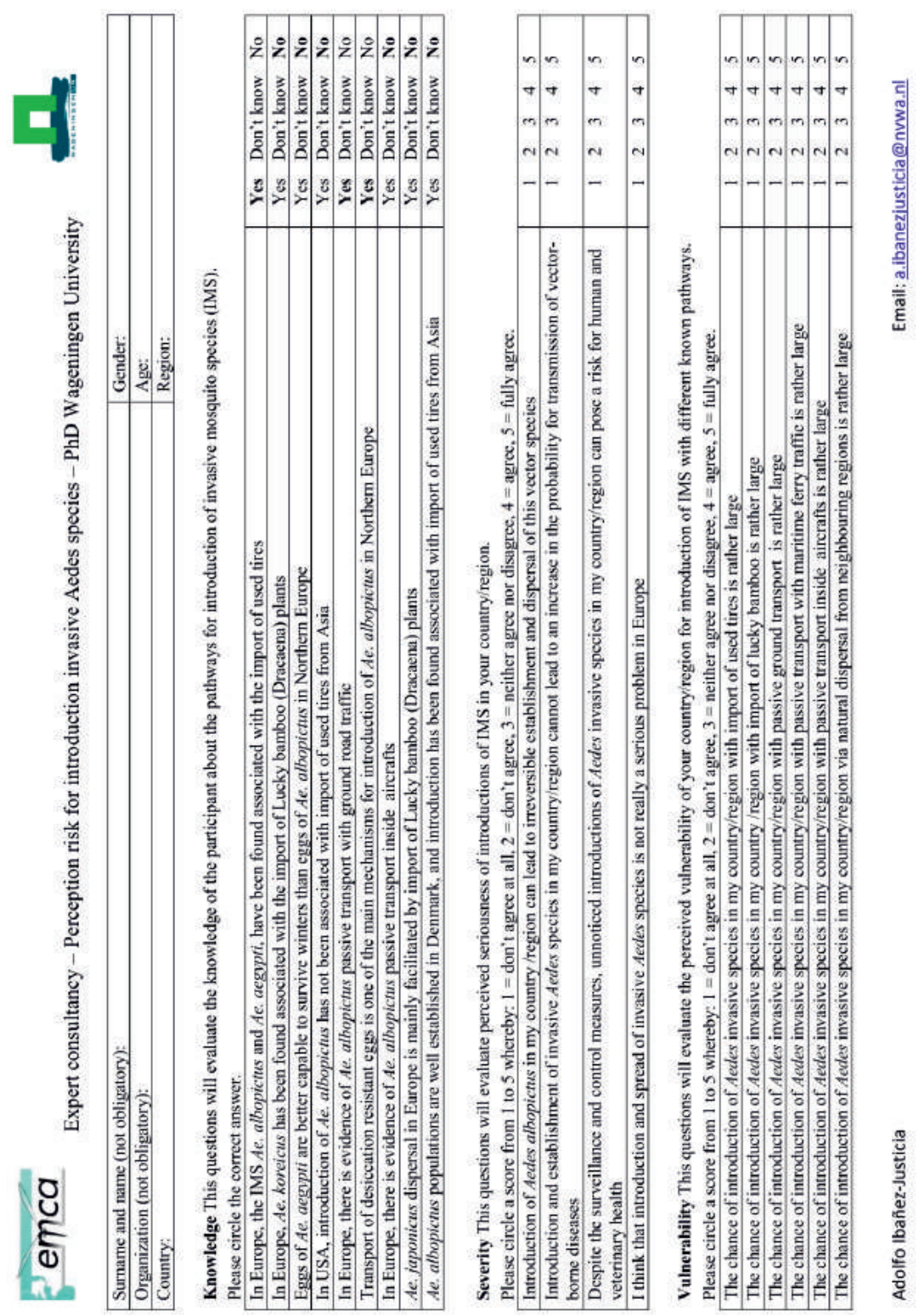



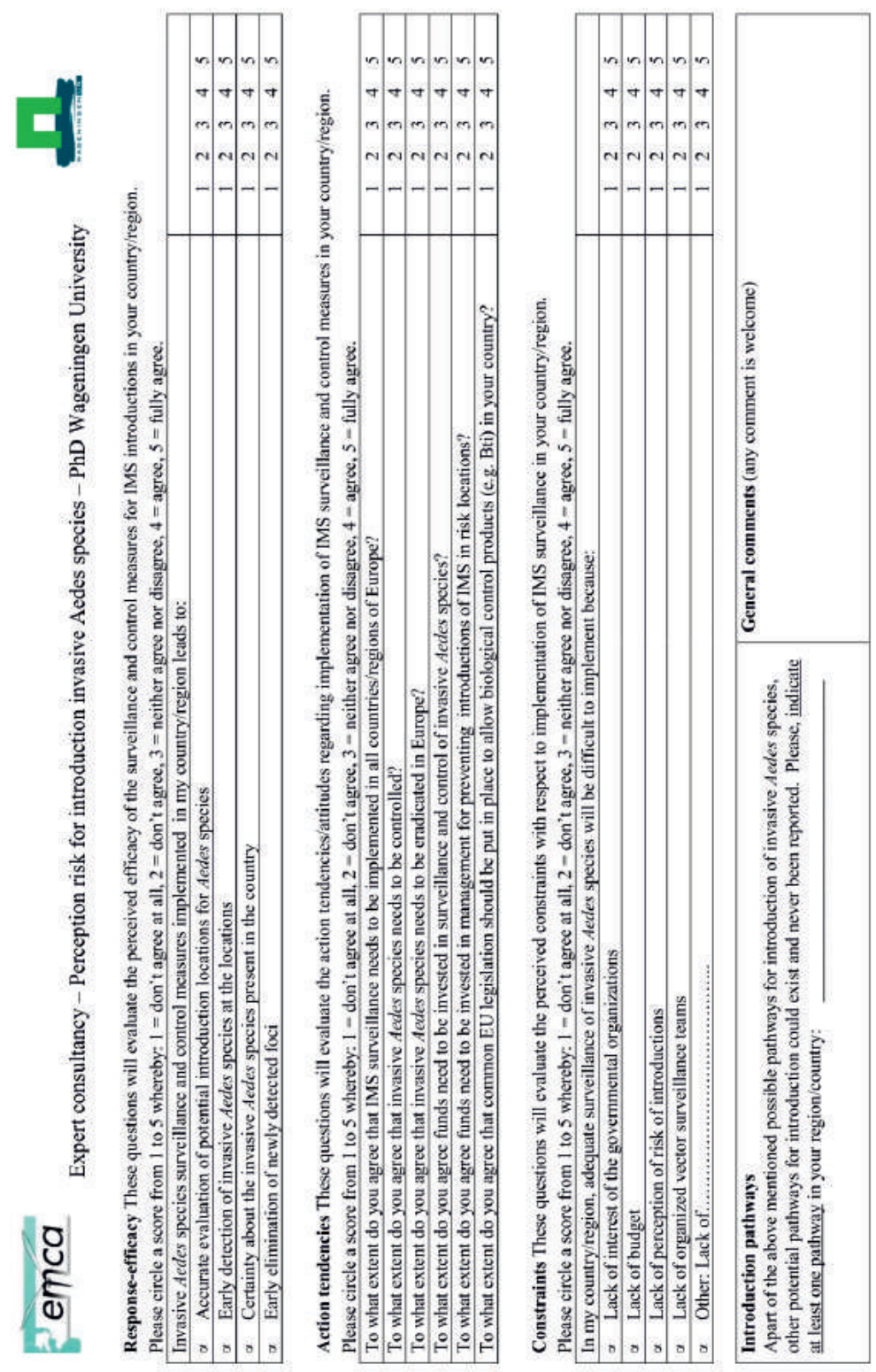

동 
S2. Scores on individual questions.
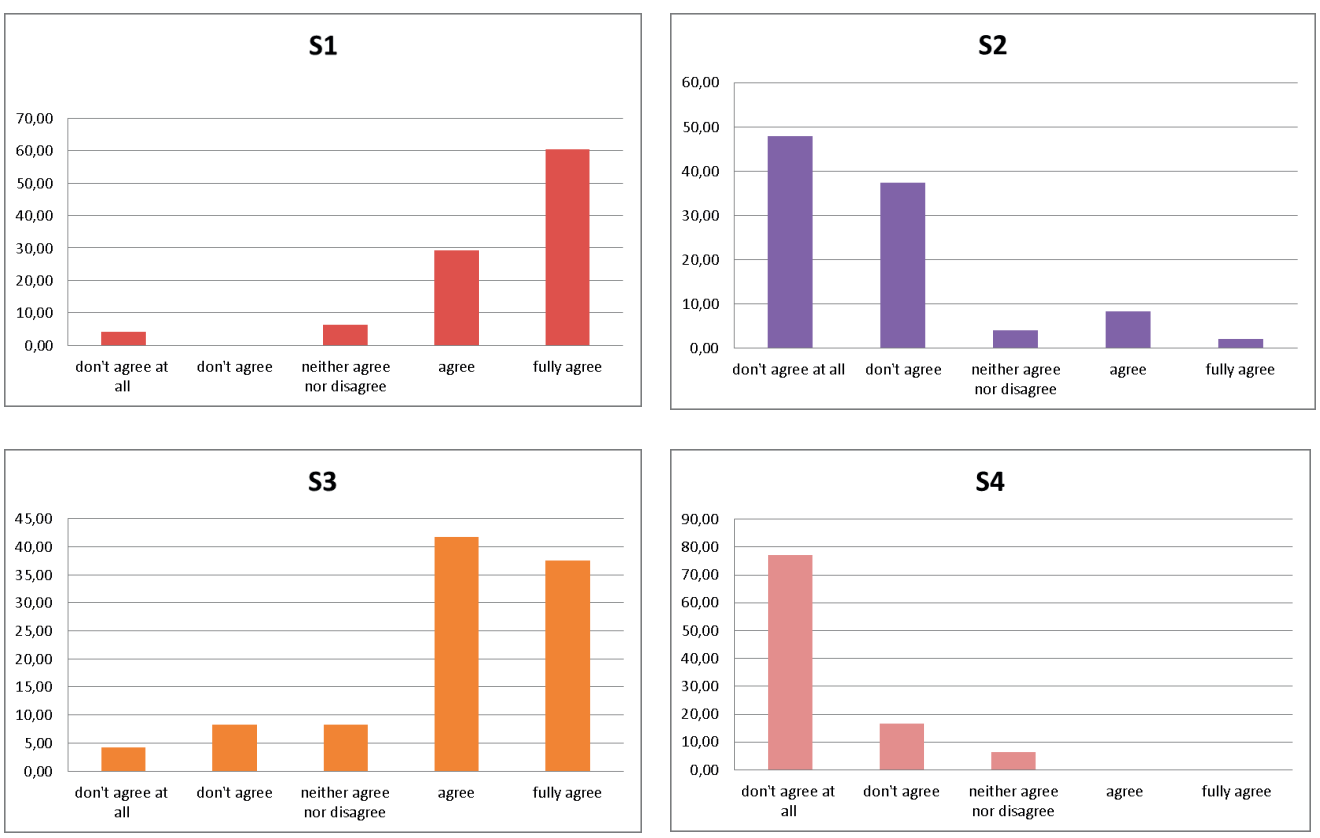

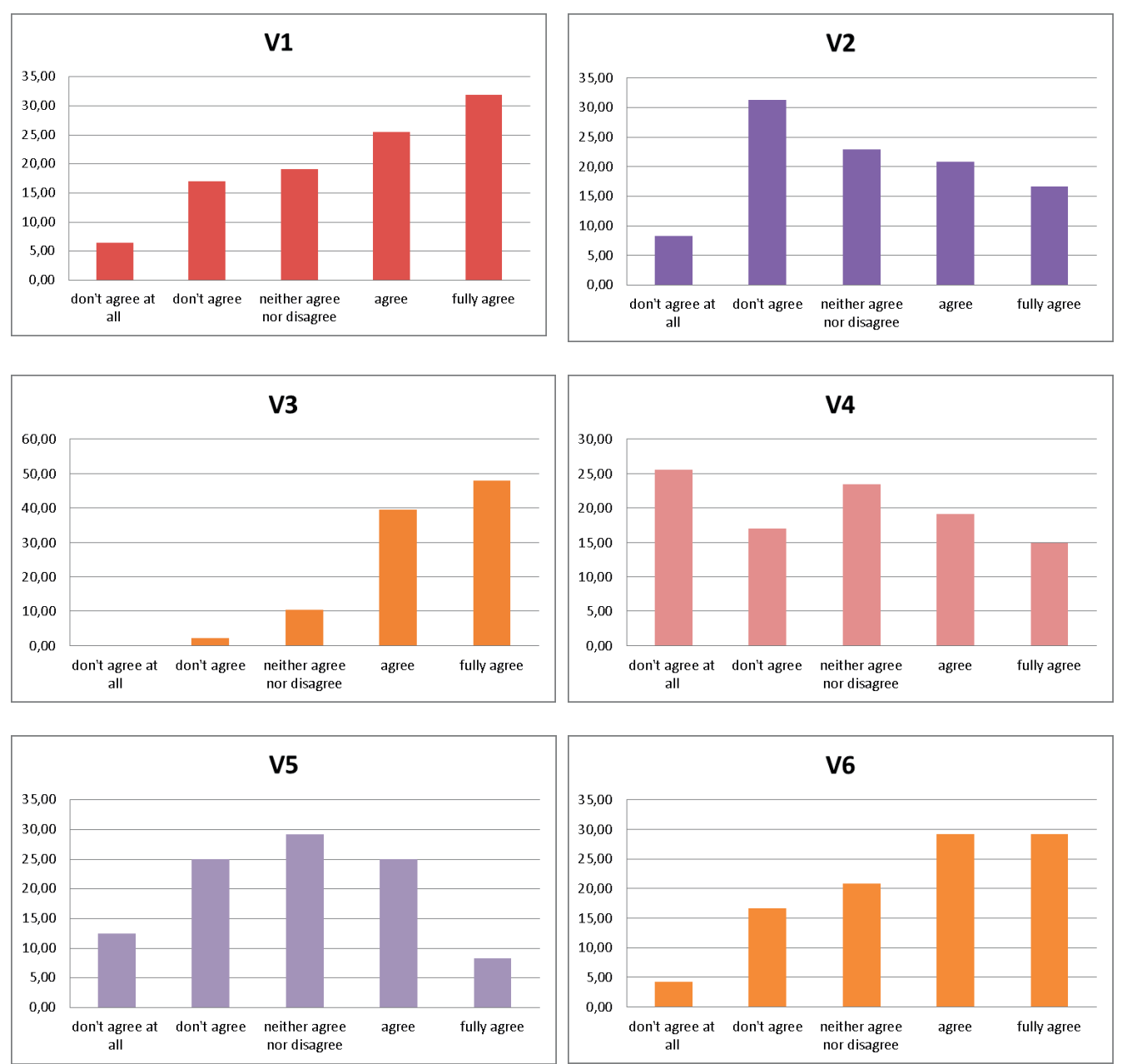

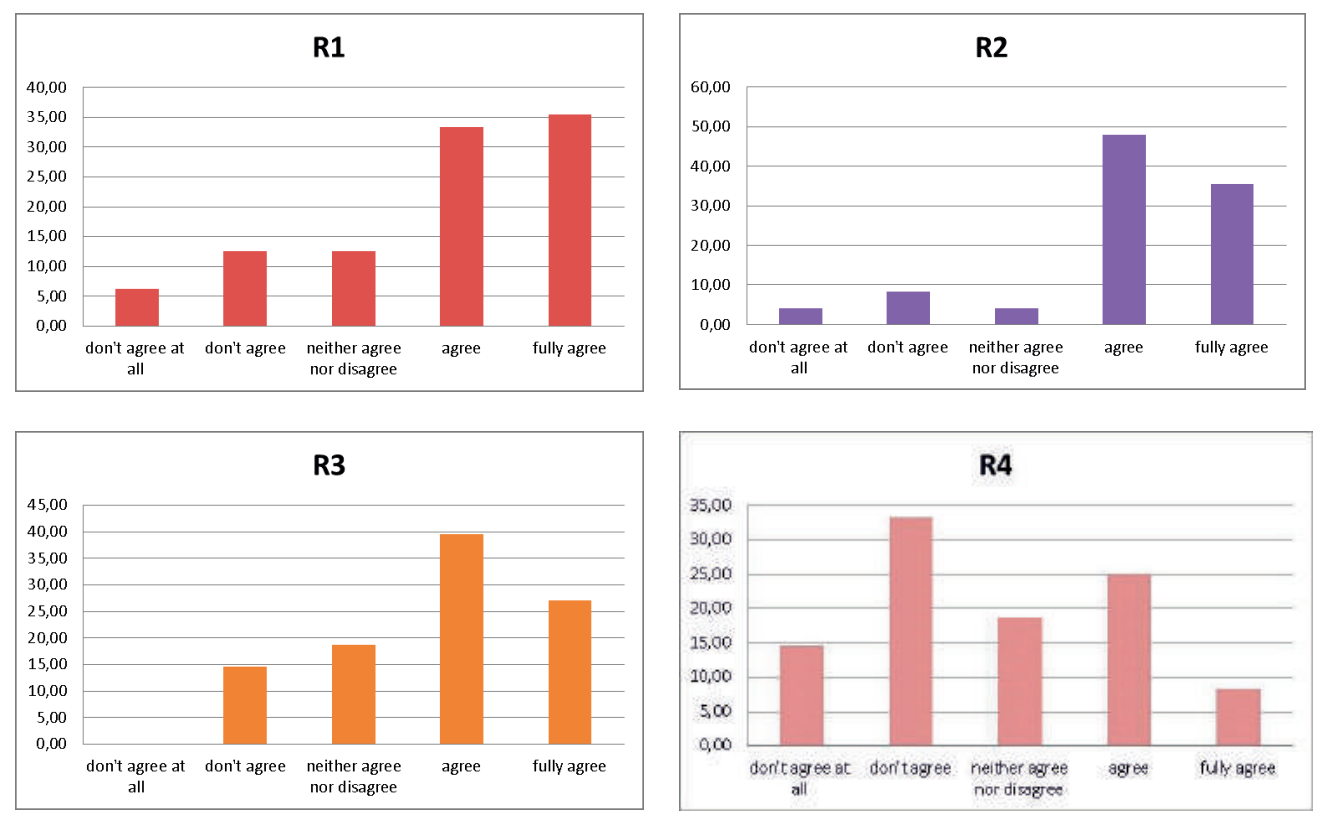

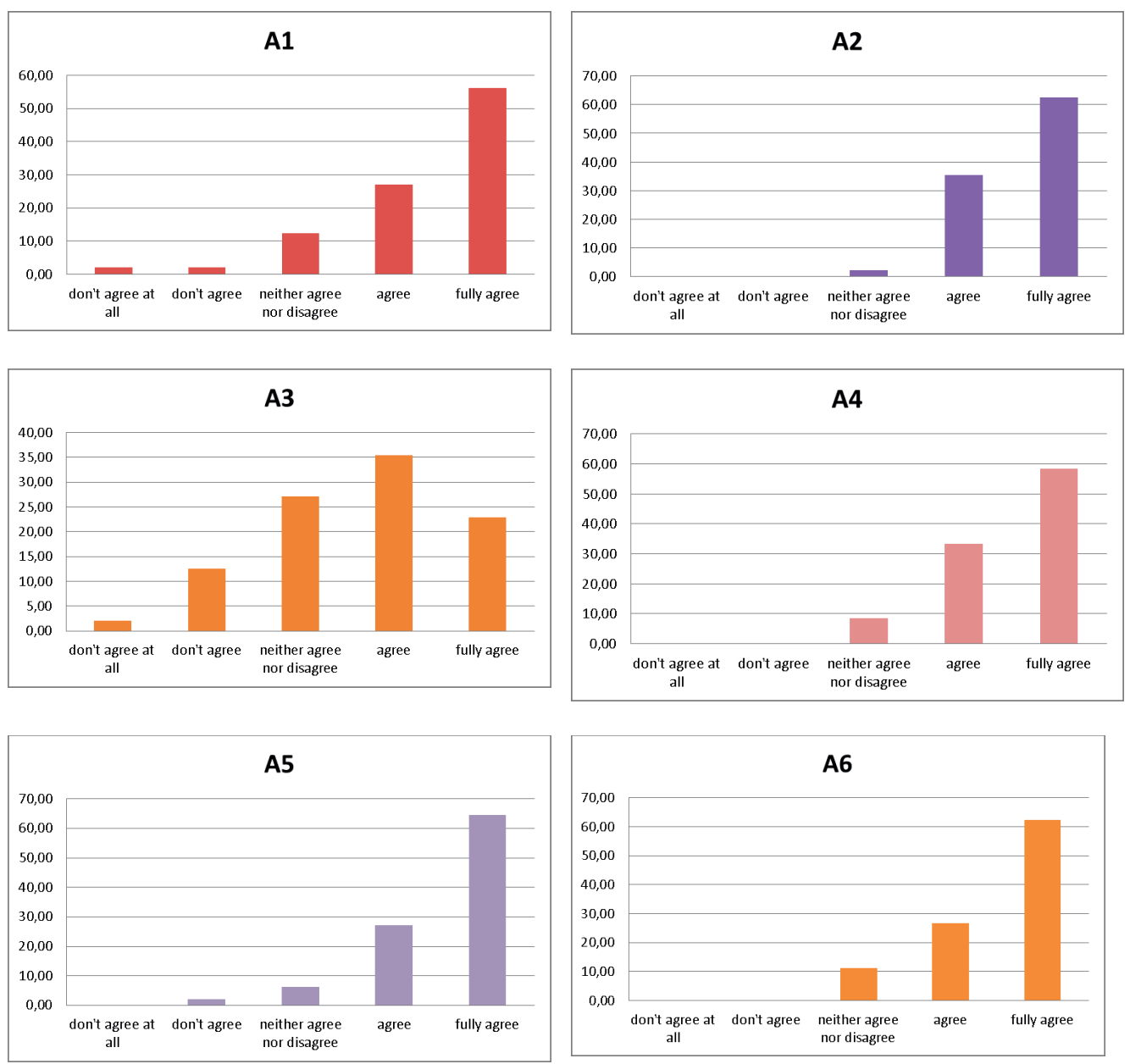
Chapter 5
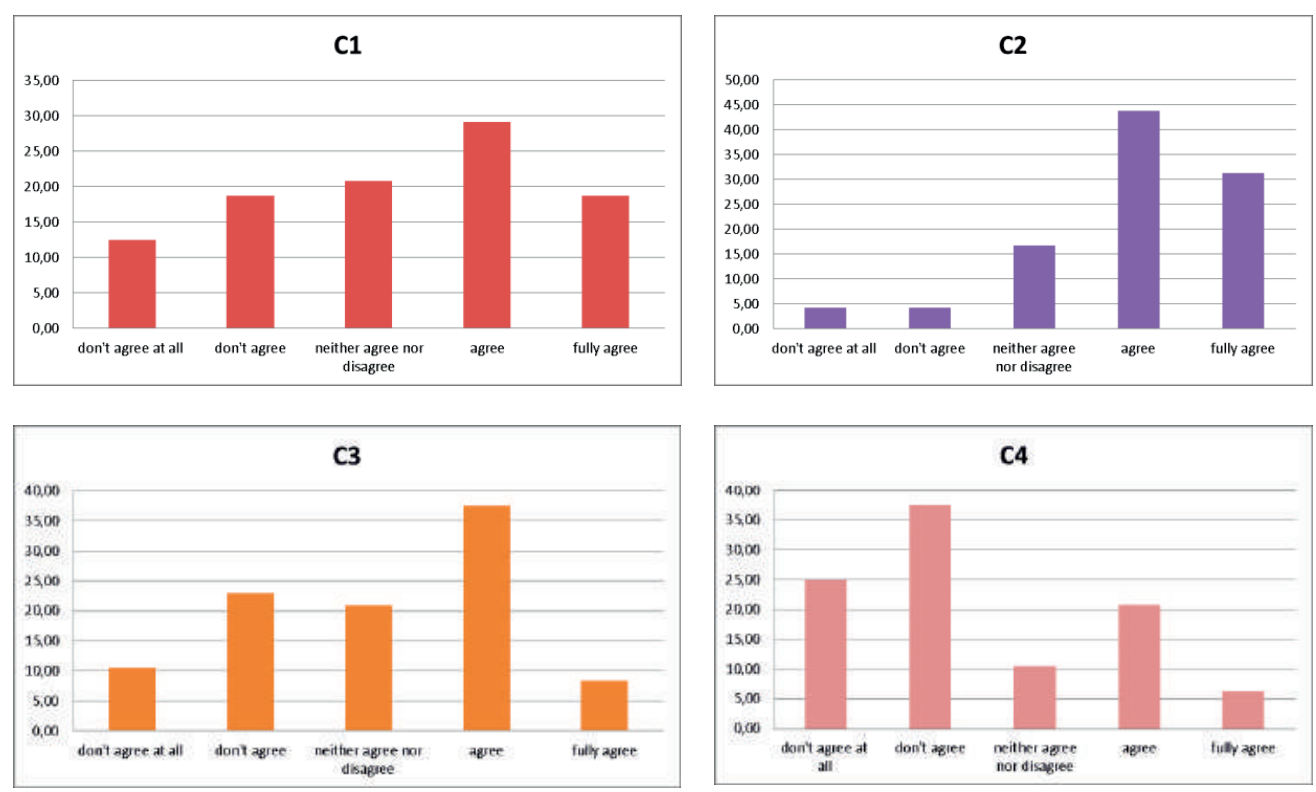



\title{
Chapter 6
}

\section{Risk-based and adaptive surveillance at Lucky bamboo and used tire importers to prevent the establishment of invasive mosquitoes in the Netherlands}

\author{
Adolfo Ibáñez-Justicia ${ }^{1}$, Constantianus J.M. Koenraadt ${ }^{2}$, Arjan Stroo $^{1}$, Ron van Lammeren ${ }^{3}$, \\ and Willem Takken ${ }^{2}$
}

1. Centre for Monitoring of Vectors, Netherlands Food and Consumer Product Safety Authority, Wageningen, The Netherlands.

2. Wageningen University \& Research, Department of Plant Sciences, Laboratory of Entomology, Wageningen, The Netherlands

3. Wageningen University \& Research, Department of Environmental Sciences, Laboratory of Geo-information Science and Remote Sensing, Wageningen, The Netherlands 


\begin{abstract}
The detection of Aedes albopictus in Lucky bamboo greenhouses in 2005 and Ae. atropalpus at used tire importers in 2009 illustrates that the Netherlands is exposed to the risk of introductions of invasive mosquito species (IMS). In order to detect introductions and avoid possible proliferation of IMS at these locations, a risk-based and adaptive surveillance has been implemented since 2010. This approach is characterized by adjustments in the sampling methodology and frequency of inspections over the course of the study. More specifically, it is informed by findings of IMS from the previous years and by the advances in detecting methods. Results of this surveillance (2010-2016) are presented, with a focus on the effectiveness of the management, monitoring and mosquito control operations. Despite the implementation of preventive measures, some imported Lucky bamboo carried Ae. albopictus eggs that developed to adults in greenhouses. One Ae. japonicus was also found in the samples from a greenhouse. At used tire companies, IMS were found at 12 out of 43 companies. Overall, results show that all findings were recorded at used tire companies considered as 'high risk' in the risk categorization. Invasive mosquito species identified were Ae. albopictus, Ae. atropalpus, Ae. aegypti, and Ae. japonicus, of which Ae. albopictus has been found every year since 2010. Actions were taken to eliminate these introductions. It is concluded that the implemented risk-based and adaptive surveillance it's an effective methodology for detection of IMS, and that application of governmental management measures in combination with mosquito control has stabilized the situation at these locations and has kept the introductions under control.
\end{abstract}

Keywords: mosquitoes, Aedes, vector control, used tires, lucky bamboo 


\section{Introduction}

Increasing international trade, together with international travel, facilitate the expansion of the native range of invasive mosquito species (IMS) (Tatem et al. 2006). Europe has witnessed several examples of the introduction of IMS in several countries. For example Aedes albopictus (Sabatini et al. 1990, Adhami and Reiter 1998, Schaffner and Karch 2000, Roiz et al. 2007, Collantes et al. 2015, Delacour et al. 2015), Ae. aegypti (Scholte et al. 2010a, Akiner et al. 2016, Ibañez-Justicia et al. 2017), Ae. atropalpus (Romi et al. 1997, Scholte et al. 2009), Ae. japonicus (Schaffner et al. 2009, Versteirt et al. 2009, Ibañez-Justicia et al. 2014, Seidel et al. 2016), and Ae. koreicus (Capelli et al. 2011, Versteirt et al. 2012, Bezzhonova et al. 2014, Suter et al. 2015, Kurucz et al. 2016, Werner et al. 2016). The introduction and possible establishment of these IMS is a risk for public health due to the ability of these IMS to transmit vector-borne diseases (VBD): Ae. aegypti is a vector of several viruses including yellow fever virus, dengue virus, chikungunya virus and Zika virus, and Ae. albopictus is a known vector of chikungunya virus, dengue virus and dirofilariasis (dog heartworm). Laboratory studies have shown the ability of Ae. atropalpus to transmit a number of viruses including La Crosse virus and West Nile virus, and Ae. japonicus has shown to be competent for the transmission of dengue and chikungunya (Schaffner et al. 2011), and to play a role in the transmission cycle of La Crosse virus (Harris et al. 2015, Westby et al. 2015, Bara et al. 2016). Established populations of IMS in Europe have also been reported to locally transmit pathogens among humans, resulting in cases of dengue and chikungunya in southern France (Gould et al. 2010, La Ruche et al. 2010, Calba et al. 2017), dengue on Madeira island (Portugal) (Sousa et al. 2012), and chikungunya outbreaks in Italy (Rezza et al. 2007, Venturi et al. 2017). It has also been suggested that countries in which invasive Aedes mosquitoes are present, could be at risk for local Zika virus transmission (Jupille et al. 2016), but this has not occurred thus far.

Since 2005, Ae. albopictus has been occasionally introduced into the Netherlands at companies that import Lucky bamboo (Dracaena sanderiana) plants (Scholte et al. 2007). The finding of Ae. atropalpus in 2009 at two companies that import used tires also illustrates that the Netherlands is exposed to the risk of IMS imported via the international used tire trade. Prior to 2009, there are no reports of IMS imported into the Netherlands via used tires, although data collected in 2009 and 2010 suggest that it could have happened before the first reported finding (Scholte et al. 2009). From 2010 onwards, Ae. albopictus, Ae. aegypti and Ae. atropalpus have been found at used tire companies (Scholte et al. 2010a, Scholte et al. 2012), and in 2016 Ae. aegypti was found at Schiphol airport, presumable introduced by aircrafts (Ibañez-Justicia et al. 
2017). To date, these species have not established populations in the country. The only established population of an IMS, Ae. japonicus, is found in the municipality of Lelystad since 2012 (Ibañez-Justicia et al. 2014). It is not known how long this species has been present in that locality prior to 2012 .

The policy of the government of the Netherlands is to prevent the establishment of IMS or to postpone their establishment as long as possible. Several public health responses have been formulated for the Netherlands. First, after signing covenants with the Lucky Bamboo sector branch in 2007, and a temporary Commodities Act Decree in 2009, the Government of the Netherlands approved the Lucky Bamboo Commodities Act Decree in April 2011. This Act provides preventive measures for Lucky bamboo import with the aim of reducing the risks to public health (Staatsblad van het Koninkrijk der Nederlanden 2011, 2014). Two control measures using biocides are included. First, containers and boxes with plants need to be opened at the company in a separate room, and an adequate treatment with a biocide has to be applied to kill Ae. albopictus adults that might be present inside the containers and boxes. Second, during the period that Lucky Bamboo is present in the greenhouse, water used for the plants has to be treated with a biocide to eliminate mosquito larvae. Similarly, used tire companies and the Government of the Netherlands signed a covenant for implementation of measures to prevent or limit repeated introductions of IMS at these companies (Staatsblad van het Koninkrijk der Nederlanden 2013, 2016). These measures include (i) the handling and separate storage of tires from 'risk regions' (as defined by ECDC mosquito maps (ECDC 2017)), (ii) identification of these tires so they can be tracked, (iii) dry import and dry storage of all imported tires from risk regions, and (iv) accelerating the processing or disposal of these tires at the company.

Here, we present the results of descriptive studies on a risk-based and adaptive national surveillance of IMS implemented in Lucky bamboo greenhouses and used tires companies in the Netherlands, with a focus on the effectiveness of the management and the mosquito monitoring and control operations for the years 2010-2016. In addition, we present a revised methodology to assign used tire companies to different risk categories for the import of IMS. Finally, we report findings of accidental introductions of IMS reported by citizens in urban areas in 2016. 


\section{Methods}

\section{Identification of risk locations}

Based on the European Centre for Disease Prevention and Control guidelines (ECDC 2012), IMS surveillance has been carried out annually in the Netherlands from 2010 onwards at potential risk locations. Two potential risk locations were identified: Lucky bamboo greenhouses and used tire companies.

Lucky bamboo greenhouses: Phytosanitary inspection of imported plants is one of the activities of the Netherlands Food and Consumer Product Safety Authority (NVWA). NVWA was asked to provide the locations where Lucky bamboo was imported, processed and/or maintained. All traders importing Lucky bamboo of Asian origin were considered potential locations for introduction of IMS and were included in the survey.

Used tire companies: In cooperation with representatives of the tire industry VACO (VACO Bedrijfstakorganisatie voor de banden- en wielenbranche 2016), the risk of used tire companies for mosquito introduction was categorized, and locations of companies were provided. Additional information of used tire companies (not member of VACO) was searched by systematically identifying tire traders via the internet. A questionnaire, including information on the potential risk of IMS and why these are likely to be introduced with used tires, was sent to companies that were registered as trading in used tires. The aim was to acquire information regarding risk factors of imported tires. Companies that did not respond to the questionnaire were contacted by telephone and asked the same questions as in the questionnaire. From 2010 onwards, a quantitative risk assessment was performed every year for each company to assess the risk of introduction of IMS. All approached companies that imported used tires were included in the categorization. For this assessment, the following risk factors were taken into account (see Table 1 and Figure 1): (1) the type of tires that were imported, (2) the country of origin of the tires, (3) the method of storage, (4) results of findings of IMS during previous surveillance activities and (5) risk assessment by the inspectors. The fifth risk factor assessed the perception of the inspector for risk of IMS introduction after visiting the used tires facilities (e.g. 'low risk' perceived in case domestic agricultural tires have been stored outdoors for several years). Companies were visited by the inspectors before the start of the surveillance to verify the data received in the questionnaire, to make appointments with the responsible employees for further monitoring activities conducted by the inspectors, and to define the area on the property that was used to store used 
tires. This area was verified using a recent aerial picture of the location. The scores are relative values used to quantify the risk of importing IMS for the different used tire companies. Table 1 is updated from the table presented in Beeuwkes et al. (2011).

Table 1. Risk factors and their relative scores, as used to categorize the risk of introduction of IMS at used tire companies in Table 3. The total score of an used tire company was the sum of the individual scores of the five risk factors. The risk factors 'Previous results' and 'Risk assessm ent inspectors' were only applied from 2011 onwards.

\begin{tabular}{llc}
\hline \hline Risk factor & Description & Relative score \\
\hline 1. Type of tires & Car/van & 0 \\
& Truck & 1 \\
& Airplane & 1 \\
& Agricultural vehicles & 1 \\
2. Origin & A combination of tire types above & 1 \\
& From EU country regions without reports of exotic mosquitoes & 0 \\
& From EU country regions reported as positive & 2 \\
& From Dutch company reported as positive & 2 \\
3. Storage method & From non-European country & 2 \\
& A combination of origins above & 2 \\
& Indoors (dry) & 0 \\
4. Previous results & Outdoors (exposed to rain) & 2 \\
5.Risk assessment inspectors & Inspector observations indicate a low risk & 2 \\
& A combination of storage methods above & 5 \\
\hline
\end{tabular}

\section{Mosquito surveillance}

Inside all Lucky bamboo greenhouses, one BG-Sentinel trap (BGS, Biogents AG, Regensburg, Germany) and one Mosquito Magnet Liberty Plus trap (MM-trap, $\mathrm{CO}_{2}$ baited, Woodstream Corporation, Lilitz, PA, USA) ran continuously during the entire year (1 January - 31 December). Both traps were placed next to each other. BGS was used with a specific dispenser (BG-Lure cartridge), which contains a lure that is a combination of volatile compounds that are also present on human skin (ammonia, lactic acid and caproic acid). MMtraps operate by the release of $\mathrm{CO}_{2}$ and heat as mosquito attractant (Guerenstein and Hildebrand 2008). These traps are advised for routine surveillance of Ae. albopictus elsewhere (Hoel et al. 2009). In addition, from May to October, a minimum of three oviposition traps (ovitraps) were also deployed immediately outside the greenhouses. This was done to detect Aedes IMS that may have left the indoor environment of the greenhouse and forage for suitable oviposition sites outdoors. Ovitraps were small black plastic buckets, 
filled with hay-infusion and a piece of polystyrene as oviposition support (Velo et al. 2016). Samples from all traps used were collected every two weeks. If during the course of the study IMS were detected in any of the deployed traps, larval and adult searches were performed at the greenhouse using fine mesh aquarium nets for larvae and aspirators for adults. These samples did not follow a regular scheme and were taken on a voluntary basis by the inspectors.

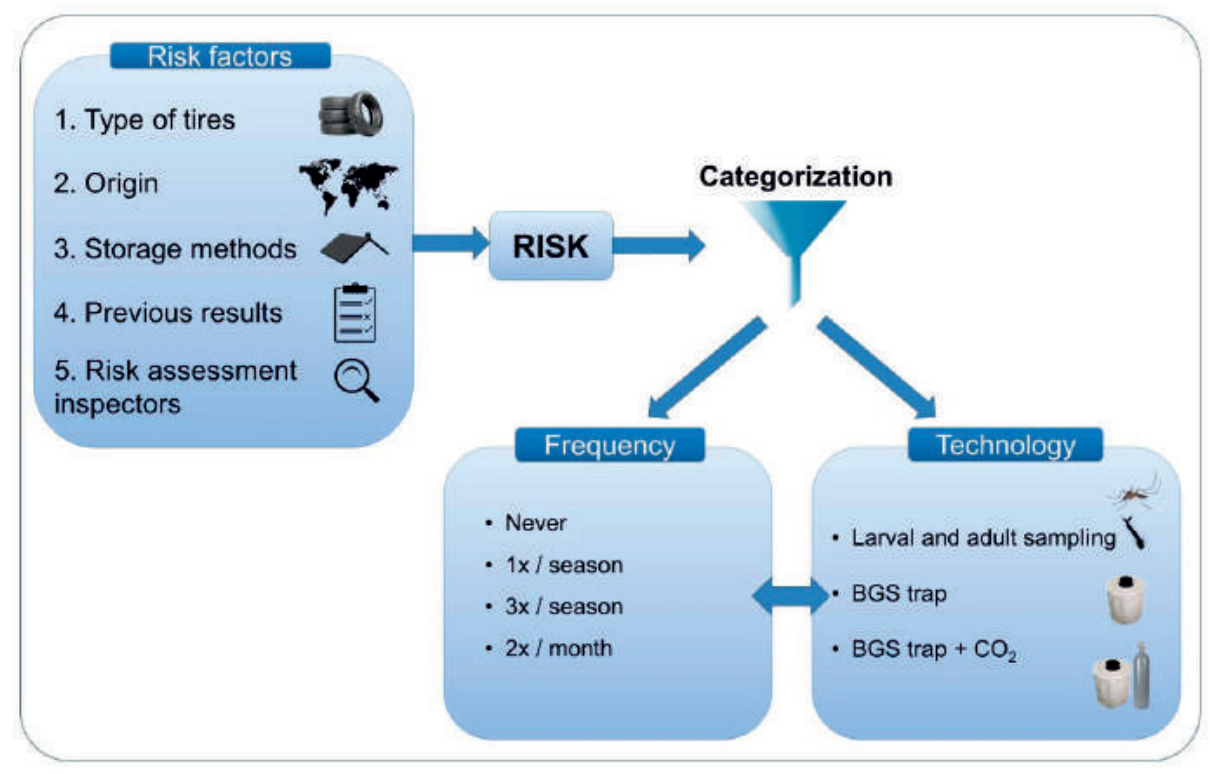

Figure 1. Flowchart of risk based and adaptive surveillance for used tire companies.

At used tire locations, manual larval and adult searches, and mosquito trap sampling were carried out every two weeks during the period in which mosquitoes in the Netherlands are most active, i.e. from mid-April to mid-October. For larval sampling, between 50 and 100 used tires, accessible for the inspector and distributed over the property of the company, were inspected for the presence of mosquito larvae during inspection. When adults of IMS were detected during larval sampling, these were collected using sweep nets and aspirators. Surveillance for IMS at used tire companies was done with BGS traps, lured with BG-Lure and $\mathrm{CO}_{2}$ (http://www.bg-sentinel.com). Sampling method and frequency of inspections performed each year are summarized in Table 3. The surveillance strategy was adapted over the course of the study, mostly informed by findings of IMS from the previous years and by the advances in IMS detecting methods. For example, less labour intensive and more 
sensitive collection methods, such as BGS traps with $\mathrm{CO}_{2}$, replaced the visual inspection of tires for larval sampling as a main method for detecting IMS at the high risk companies from 2013 onwards.

Following our adaptive approach, if an IMS was detected during the surveillance within the used tires company limits, more intensive inspections were initiated here, as well as within a predefined perimeter of $500 \mathrm{~m}$ from the limits of the company, where used tires can be stored (indoors or outdoors, see Figure 2). This consisted of increased frequency of deployment of traps (from every two weeks to every week), increased number of traps (minimum of three traps outside the limits of the company), as well as sampling and subsequent removal of potential larval habitats for container-breeding Aedes. If an IMS was detected during the surveillance within the $500 \mathrm{~m}$ perimeter, but outside the company limits, the sampling area was increased by including a new circular search area of 500m radius from the new finding site (see Figure 2). Two BGS traps were deployed, and sampling and subsequent removal of potential larval habitats for container-breeding Aedes was performed in the increased area. The increase of the sampling area at a location stopped when no more IMS specimens were detected outside the company. Due to the fact that many potential breeding sites were available outside the company limits, oviposition traps were not used. Rather, larval sampling of potential breeding sites complemented with BGS trap sampling were implemented.

\section{Sample identification}

Samples were labelled in the field with a unique code, sealed, and sent to the laboratory of the Centre for Monitoring of Vectors of the National Reference Centre (CMV-NRC-NWVA) for species identification. Mosquitoes were counted and morphologically identified using the morphological electronic key of Schaffner (Schaffner et al. 2001), and the morphological key of Becker (Becker et al. 2010). Samples containing only indigenous mosquito species were considered as 'negative samples' and samples containing IMS were considered as 'positive samples'. The abundance of indigenous mosquitoes was recorded from 2013 onwards. Each mosquito that was morphologically identified as an IMS was double-checked by a different mosquito specialist. From 2014 onwards, IMS identifications were also validated by using a real-time PCR specifically developed for the surveillance for detecting Ae. albopictus, Ae. aegypti, Ae. atropalpus, and Ae. japonicus (van de Vossenberg et al. 2015). 


\section{IMS control}

As stipulated in the Lucky Bamboo commodity act (Staatsblad van het Koninkrijk der Nederlanden 2011), importers need to apply mosquito larvicide to the water used to grow plants during the time that the plants are kept in the greenhouse. Additionally, if during surveillance an IMS was found in any of the traps, the importer was notified by the NVWA and was advised to apply adulticide in the greenhouse.

In case an IMS was detected at a used tire company, the site was treated by spraying Bacillus thuringiensis israelensis (Bti) (dilution rate of 1,5ml/100ml water) (VectoBac WG, Valent BioSciences LLC, Libertyville, IL 60048, USA) against larvae inside the tire stacks and deltamethrin (dilution rate of 2,5ml/100ml water) (aqua K-Othrine, Bayer Environmental Sciences, 51373 Leverkusen, Germany) against adult mosquitoes. Larval control of the surrounding area (predefined perimeter of $500 \mathrm{~m}$ ) consisted of removal of potential larval breeding sites for container-breeding Aedes spp., or treatment with either Bti space spray (VectoBac WG, Valent BioSciences), or with Bti/Bacillus sphaericus (Bs) granules (Vectomax FG, Valent BioSciences). One week later, adulticiding was repeated once. Larviciding was repeated every two to three weeks until the end of October, when ambient conditions for IMS became generally unfavorable.
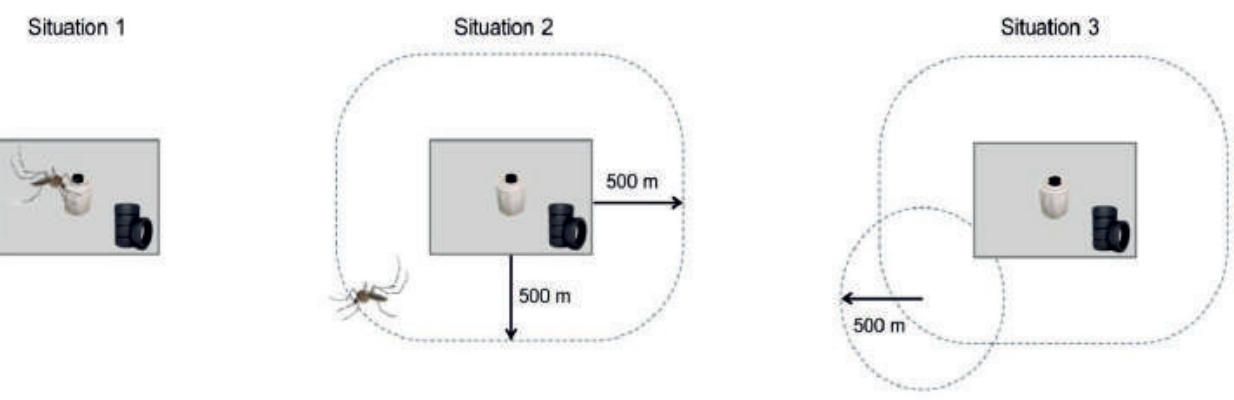

Figure 2. Spatial sampling scheme of adaptive surveillance of used tired companies. Situation 1: mosquito sampling inside the used tires company property; Situation 2: mosquito sampling outside the property of used tires companies after finding and IMS inside the company property; Situation 3: extension of surveillance area in case of detection of an IMS within the initial $500 \mathrm{~m}$ zone. 


\section{Data analyses}

All data from each sampling location were included in VecBase (a tailor-made Oracle database application), with details on $\mathrm{X}-\mathrm{Y}$ coordinates, unique barcoded sample number, field inspector name, sampling period, species captured, number of individuals, sex (male, female), etc. VecBase provides options to manage the stored data and to make specific queries (ECDC 2012). Data on the annual numbers of imported used tires from 2010 until 2016 were provided by the CBS (CBS-Statistics-Netherlands 2017). Data on the annual numbers of imported Lucky bamboo plants from 2010 up to 2016 was provided by the phytosanitary authorities of the Netherlands (NVWA).

In order evaluate the effect of the adaptive surveillance strategy in changes in the number of IMS positive samples at the used tire companies over time, a subset of the collected data was further analyzed. This subset consisted of samples taken inside the used tires companies that were categorized as 'high risk' for introductions and that used the same sampling methodology (BGS-trap with $\mathrm{CO}_{2}$ ) from 2013 to 2016. We calculated the annual proportion of the number of samples containing IMS (from the total number of samples taken) for these years of the study. A binomial test was carried out in SPSS to study the diferences between the years (SPSS Inc. Released 2008. SPSS Statistics for Windows, Version 17.0. Chicago: SPSS Inc). This test was also used to evaluate to study the diferences between the years on the number of IMS positive samples at the Lucky bamboo greenhouses. In this case, the proportion of the number of samples containing IMS (from the total number of samples) using only BGS-trap and MM-traps was used.

\section{Results}

Surveillance in Lucky bamboo greenhouses

A total of 13 unique locations where Lucky bamboo is imported were identified in the Netherlands between 2010 and 2016. Apart from one location in the province of NoordBrabant, all the other locations are situated in the West of the Netherlands in the provinces of Noord-Holland and Zuid-Holland. From 2010 to 2016, 3,022 samples were taken at Lucky bamboo greenhouses (Table 2). These mostly consisted of adult captures from the BGS and MM-traps, and eggs and larvae in the ovitraps. Invasive Aedes species detected during the surveillance period were Ae. albopictus and Ae. japonicus. Since 2010, interceptions of Ae. albopictus occurred every year, with 2010 and 2012 being the years with most locations 
found positive for this species $(n=6)$, and 2015 the year with the highest percentage of positive samples (4.1\%) (see Table 2 and Figure 3). Annually, the number of Ae. albopictus captured varied strongly (e.g. 82 captured in 2010, and two specimens captured in 2014). At the same time, the total number of imported plants as reported to the phytosanitary authorities strongly decreased from 2011 onwards (see Figure 1). However, this did not seem to coincide with a decline in the number of positive samples containing Ae. albopictus intercepted every year. A binomial test indicated that the proportion of positive samples obtained using only BGS-traps and MM-traps in 2013 (0.93\%) and 2014 (0.75\%) was significant lower than in 2010 (2.31\%), 2011 (3.11\%), 2012 (3.69\%) and 2015 (4.09\%), p<0.01 (1-sided).

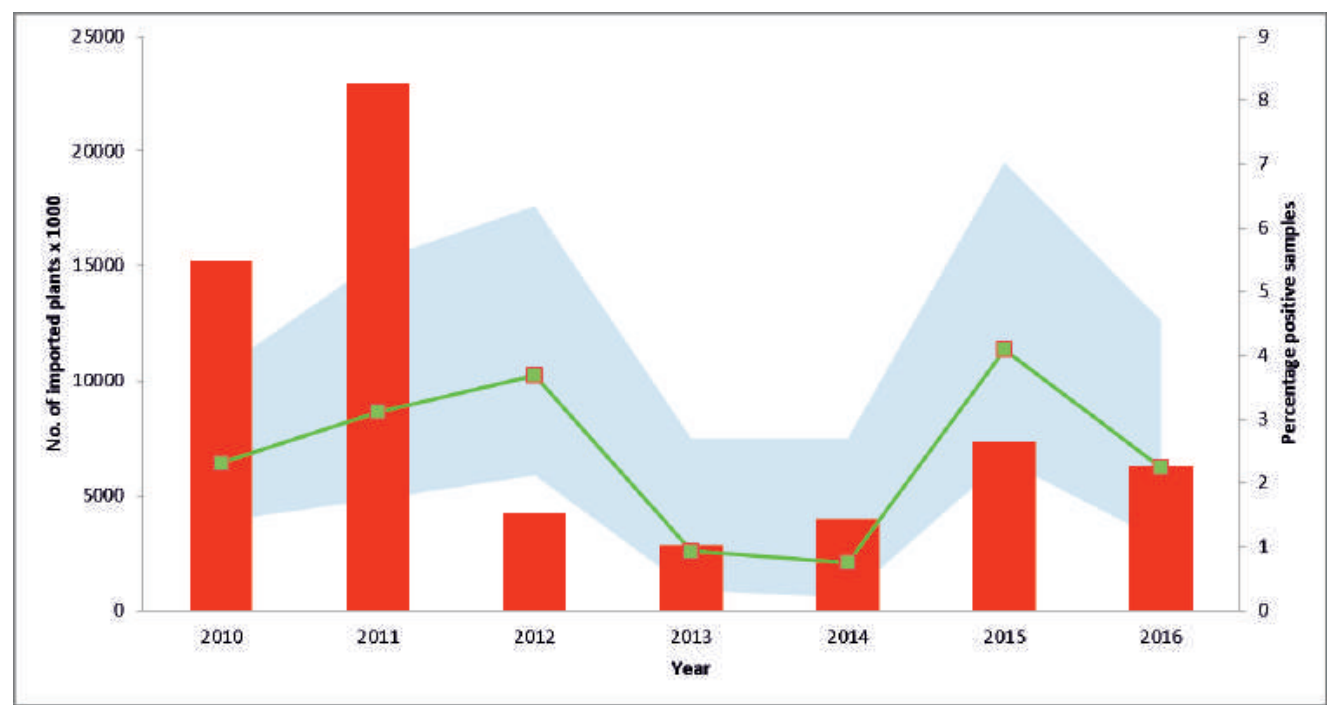

Figure 3. Number of imported Lucky bamboo plants in the Netherlands 2010-2016 (red bars, Source NVWA), and percentage of positive samples using BGS and MM traps (green line). Blue area represents $95 \%$ confidence interval of the percentage of positive samples.

Table 2. Results of the Lucky bamboo monitoring in the Netherlands from 2010-2016.

\begin{tabular}{ccccc}
\hline Year & Nr. locations & $\begin{array}{c}\text { Nr. locations } \\
\text { positive }\end{array}$ & $\begin{array}{c}\text { Nr. samples total using } \\
\text { BGS and MM-traps }\end{array}$ & $\begin{array}{c}\text { Nr. samples with IMS using } \\
\text { BGS and MM-traps }\end{array}$ \\
\hline 2010 & 11 & 6 & 649 & 15 \\
2011 & 7 & 3 & 353 & 11 \\
2012 & 7 & 6 & 325 & 12 \\
2013 & 6 & 2 & 321 & 3 \\
2014 & 5 & 1 & 266 & 2 \\
2015 & 5 & 4 & 293 & 7 \\
2016 & 6 & 3 & 312 & 7 \\
\hline
\end{tabular}


A total of 200 specimens of Ae. albopictus were captured, mostly by BGS-traps $(\mathrm{n}=148)$ and MM-traps ( $\mathrm{n}=48)$. Two adult specimens were captured using aspirators (one in 2010 and one in 2016). Two larvae were captured in a container with water and Lucky bamboo at arrival at the company in 2010. Results show that both trap types capture Ae. albopictus inside Lucky bamboo greenhouses, but that BG-Sentinel traps placed next to the MM-traps outperform MM-traps in detecting Ae. albopictus. No Ae. albopictus or other IMS were found in ovitraps placed in the immediate surroundings of the greenhouses. In December 2013, one mosquito collected with a MM-trap in a Lucky bamboo greenhouse was morphologically identified as an adult female Ae. japonicus; and the identification was confirmed using real-time PCR.

\section{Surveillance at used tire companies}

The quantitative risk assessment of used tire companies assigned these locations as 'high', 'medium' or 'low risk' for introduction of IMS. Based on the outcome of previous years, the relative scores associated with the risk categories for the used tires companies were adapted each year (Table 3). Between 2010 and 2016, 43 companies that trade in used tires were identified. Results of the evaluation for years 2010-2016 are shown in Table 4. The total number of companies evaluated every year fluctuates as a result of establishment of new companies, companies changing locations or companies going out of business.

Sampling methodology and frequency of sampling at the different risk levels was adapted every year and depended on the risk category (see Figure 1). In 2010, monitoring for IMS at the tire companies was only performed by inspectors sampling for larvae inside the tires and looking for adults in the immediate vicinity of these tires. Six used tire companies were positive in 2010 for IMS, and after the findings, BGS traps and MM-traps were also used at the positive locations for further detection of IMS. In 2011 and 2012, it was decided to continue larval sampling, but in order to improve the likelihood for detecting IMS, one BGS trap was placed at the companies found positive the year before. We hypothesized that data from the BGS traps would help to promptly detect IMS in known positive used tire companies in case no IMS specimens would be found during larval sampling (e.g. inside large piles of tires that are m ore difficult to sam ple). In 2013, BGS traps placed at 'high risk' locations were upgraded with a device providing $\mathrm{CO}_{2}$ in order to improve the attractiveness of the trap for capturing of IMS (Roiz et al. 2016). In 2014, it was decided to evaluate all tire companies $(n=43)$ that had traded used tires in the Netherlands, even if the risk was 
considered very low or the number of tires and origin was not indicating any risk. This served as a starting point for the surveillance after the signing of the covenant.

Table 3. Risk category assignment for used tire companies in the Netherlands from 2010-2016, and the sampling method/frequency applied based on this assignment. The relative score is the sum of the individual scores of the five risk factors in Table 1.

\begin{tabular}{llll}
\hline \hline Year & Relative Score & Risk category & Sampling method/frequency inspections \\
\hline 2010 & $>4$ & High & Larval and adult sampling / 2x month \\
& 3 & Medium & Larval and adult sampling / 3x season \\
& $0-2$ & Low & Larval and adult sampling / 1x season \\
\hline $2011-2012$ & $>5$ & High Category 1 & BG Sentinel + Larval and adult sampling / 2x month \\
& 5 & High Category 2 & Larval and adult sampling / 2x month \\
& $3-4$ & Medium & Larval and adult sampling / 3x season \\
& $0-2$ & Low & Larval and adult sampling / 1x season \\
\hline 2013 & $>=5$ & High & BG Sentinel + CO2 / 2x month \\
& $2-4$ & Medium & Larval and adult sampling / 1x season \\
& $0-1$ & Low & No monitoring \\
\hline $2014-2016$ & $>=5$ & High & BG Sentinel + CO2 / 2x month \\
& $3-4$ & Medium & BG Sentinel / 2x month \\
& $0-2$ & Low & No monitoring \\
\hline
\end{tabular}

From 2010 to 2016 a total of 7,104 samples were taken at the used tire companies and more than 260,000 mosquito specimens were collected (see Table 5). Invasive mosquito species were found at 12 of the 43 locations (28\%). At nine locations (21\%), IMS were also found in the $500 \mathrm{~m}$ buffer zone immediately outside the premises of the company. Except for 2012, positive samples were found outside the premises of the tire companies every year. Invasive mosquito species found were Ae. albopictus, Ae. atropalpus, Ae. aegypti and Ae.japonicus, of which Ae. albopictus has been routinely found every year since 2010. Aedes aegypti was only found in 2010 and Ae. atropalpus in 2010 and 2011. Both species were no longer detected after mosquito control was applied at the locations. Aedes japonicus was found during surveillance outside a company that was located in the municipality of Lelystad, 
where an established population of this species was detected in 2013 (Ibañez-Justicia et al. 2014), and also at two used tire companies in 2014 and 2015.

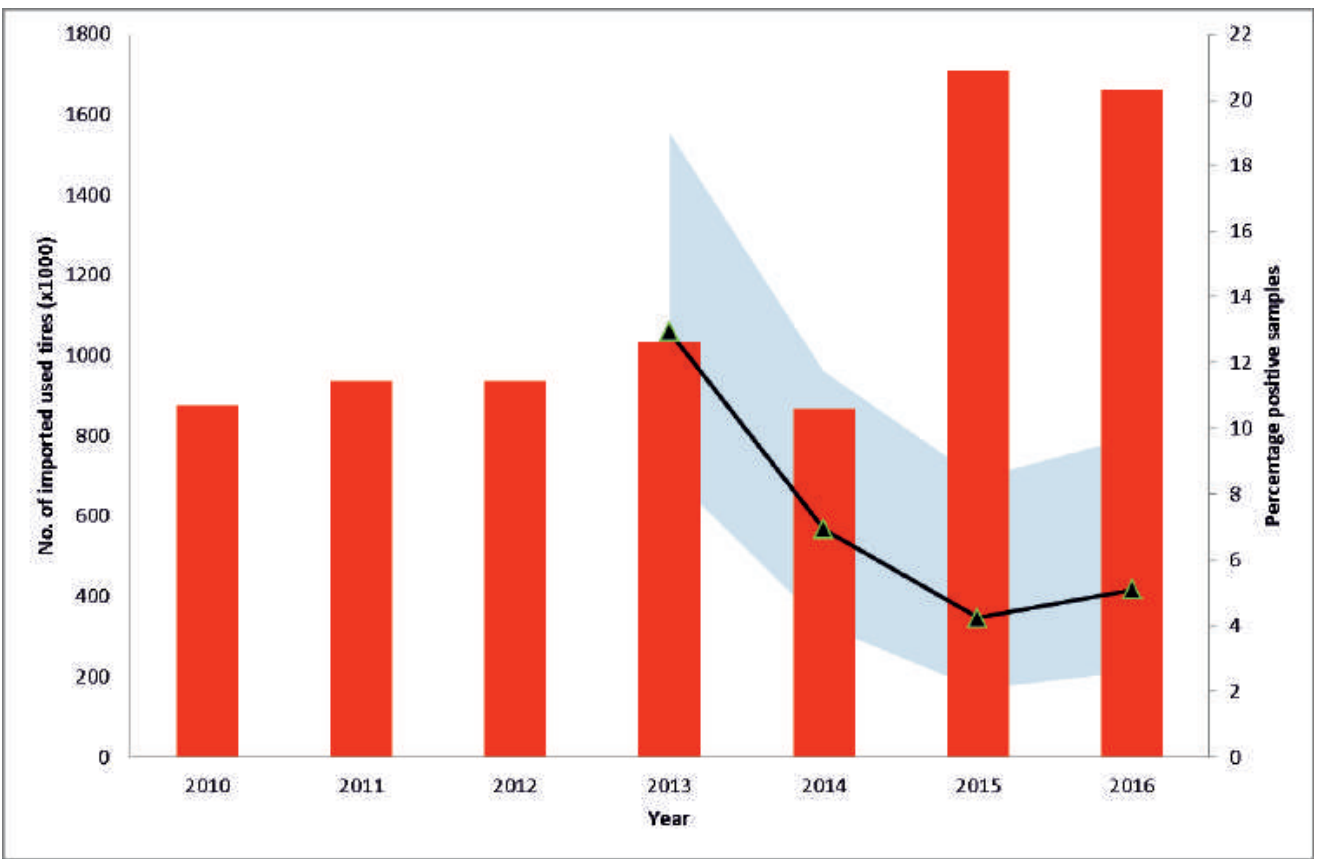

Figure 4. Number of imported used tires at the used tires companies in the Netherlands (2010-2016) (red bards, Source: $\mathrm{CBS}$ ), and percentage of positive samples using BGS-traps with $\mathrm{CO}_{2}$ (black line). Blue area represents $95 \%$ confidence interval of the percentage of positive samples.

Table 4. Risk categories of used tire companies in the Netherlands 2010-2016

\begin{tabular}{ccccc}
\hline \hline Year & Total companies evaluated & $\begin{array}{c}\text { High risk } \\
\text { (number) }\end{array}$ & $\begin{array}{c}\text { Medium risk } \\
\text { (number) }\end{array}$ & $\begin{array}{c}\text { Low risk } \\
\text { (number) }\end{array}$ \\
\hline 2010 & 34 & 14 & 3 & 17 \\
2011 & 36 & $6($ Category 1) \\
& $8($ Category 2$)$ & 4 & 18 \\
2012 & 32 & 6 (Category 1) & 6 & 10 \\
2013 & 10 (Category 2) & 11 & 6 \\
2014 & 32 & 15 & 14 & 10 \\
2015 & 43 & 13 & 7 & 1 \\
2016 & 16 & 10 & 6 & \\
\hline
\end{tabular}


In order to evaluate the impact of the adaptive surveillance strategy over time, we compared the annual proportions of samples containing IMS collected by BGS-trap with $\mathrm{CO}_{2}$ at used tire locations considered as 'high risk'. This proportion was significantly higher before the application of the covenant in 2013 (12.96\%) than in the successive three years (2014 (6.93\%), 2015 (4.24\%), 2016 (5.09\%), 1-sided binomial test, $p<0.01)$, see Figure 4.

Table 5. Number of samples processed, number of mosquito specimens processed, species and number of specimens of IMS captured at used tire companies in the Netherlands from 2010-2016. A= Adults; $P=$ Pupae; L= Larvae; $\mathrm{E}=$ eggs.

\begin{tabular}{|c|c|c|c|c|c|c|}
\hline Year & $\begin{array}{c}\text { Nr. Samples } \\
\text { (nr. samples } \\
\text { positive) }\end{array}$ & $\begin{array}{l}\text { Nr. Mosquito } \\
\text { specimens } \\
\text { (Total/A/L/P/E) }\end{array}$ & $\begin{array}{c}\text { Nr. locations } \\
\text { positive (nr. } \\
\text { positive outdoor) }\end{array}$ & $\begin{array}{c}\text { Nr. samples } \\
\text { positive inside } \\
\text { company using } \\
\text { standardized BGS- } \\
\text { trap }+\mathrm{CO}_{2}\end{array}$ & IMS name & Number $(\mathbf{A} / \mathbf{P} / \mathbf{L})$ \\
\hline 2010 & $1,116(43)$ & $\begin{array}{l}3,393(915 \mathrm{~A}, \\
2,442 \mathrm{~L}, 226 \mathrm{P}, 10 \mathrm{E})\end{array}$ & $6(3)$ & Not applicable & $\begin{array}{l}\text { Ae. atropalpus } \\
\text { Ae. aegypti } \\
\text { Ae. albopictus }\end{array}$ & $\begin{array}{l}325(121 \mathrm{~A}, 203 \mathrm{~L}, 1 \mathrm{P}) \\
13(13 \mathrm{~A}) \\
23(15 \mathrm{~A}, 8 \mathrm{~L}) \\
\text { Total 361 }\end{array}$ \\
\hline 2011 & $1,466(14)$ & 864 (52A, 809L, 3P) & $4(2)$ & Not applicable & $\begin{array}{l}\text { Ae. atropalpus } \\
\text { Ae. albopictus }\end{array}$ & $\begin{array}{l}22(21 \mathrm{~A}, 1 \mathrm{~L}) \\
30(22 \mathrm{~A}, 5 \mathrm{~L}, 3 \mathrm{P}) \\
\text { Total 52 }\end{array}$ \\
\hline 2012 & $752(10)$ & $\begin{array}{l}7,738(5103 \mathrm{~A}, \\
2638 \mathrm{~L}, 3 \mathrm{E})\end{array}$ & $5(0)$ & Not applicable & Ae. albopictus & $\begin{array}{l}27(27 \mathrm{~A}) \\
\text { Total } 27\end{array}$ \\
\hline 2013 & $1,137(31)$ & $\begin{array}{l}24,657(12415 \mathrm{~A}, \\
12238 \mathrm{~L}, 4 \mathrm{P})\end{array}$ & $9(7)$ & 21 & $\begin{array}{l}\text { Ae. albopictus } \\
\text { Ae. japonicus }\end{array}$ & $\begin{array}{l}74(46 \mathrm{~A}, 28 \mathrm{~L}) \\
1(1 \mathrm{~A}) \\
\text { Total } 75\end{array}$ \\
\hline 2014 & $1,150(18)$ & $\begin{array}{l}64,163(53,008 \mathrm{~A}, \\
11,047 \mathrm{~L}, 108 \mathrm{P})\end{array}$ & $6(1)$ & 12 & $\begin{array}{l}\text { Ae. albopictus } \\
\text { Ae. japonicus }\end{array}$ & $\begin{array}{l}31(31 \mathrm{~A}, 16 \mathrm{~L}) \\
1(1 \mathrm{~L}) \\
\text { Total } 48\end{array}$ \\
\hline 2015 & $863(9)$ & $\begin{array}{l}45,292(18,509 \mathrm{~A}, \\
26,472 \mathrm{~L}, 311 \mathrm{P})\end{array}$ & $6(1)$ & 7 & $\begin{array}{l}\text { Ae. albopictus } \\
\text { Ae. japonicus }\end{array}$ & $\begin{array}{l}11(11 \mathrm{~A}) \\
1(1 \mathrm{~A}) \\
\text { Total 12 } \\
\end{array}$ \\
\hline 2016 & $620(12)$ & $\begin{array}{l}118,364 \\
(109,403 \mathrm{~A}, 527 \mathrm{E}, \\
8,254 \mathrm{~L}, 180 \mathrm{P})\end{array}$ & $4(1)$ & 8 & Ae. albopictus & $\begin{array}{l}14(14 \mathrm{~A}) \\
\text { Total } 14\end{array}$ \\
\hline Total & 7,104 & 264,471 & $12(9)$ & & $\begin{array}{l}\text { Ae. aegypti } \\
\text { Ae. atropalpus } \\
\text { Ae. albopictus } \\
\text { Ae. japonicus }\end{array}$ & $\begin{array}{l}13 \text { (1 year) } \\
347 \text { (2 years) } \\
210 \text { ( } 7 \text { years) } \\
3 \text { (3 years) } \\
\text { Total }: 573\end{array}$ \\
\hline
\end{tabular}

Besides the detection of IMS at Lucky bamboo and used tire sites, there were two situations involving Ae. albopictus outside these locations in 2016 (see Table 7). First, at the end of June that year, nuisance from Ae. albopictus was reported at a used truck company in the municipality of Weert. Inspections and deployed traps discovered a population of Ae. albopictus inside and outside the property of the company. Adulticiding and larviciding was applied to control this focus. Its location is in the same industrial area of a used tire company (Weert), but no trading or other relations were identified between the two companies.

Furthermore, the used tire company in Weert has a BGS-trap with $\mathrm{CO}_{2}$, and the last positive sample in this trap was two years earlier (June 2014). In total, 18 Ae. albopictus specimens 
were found during surveillance (seven adults, eight larvae and three pupae). Later in August 2016, an adult female Ae. albopictus was reported by a citizen in an urban area in the municipality of Veenendaal. Immature stages of Ae. albopictus were found breeding in artificial containers present in the backyards of the houses. Mosquito control based on the use of larvicides (Vectomax FG) was immediately applied to eliminate this focus. In total, in the 545 samples taken in the area with BGS-traps and larval sampling, 74 Ae. albopictus specimens were found during this surveillance (26 adults, 44 larvae and 4 pupae).

Table 6. Results of monitoring and control of IMS in the Netherlands 2010-2016. (*) finding outdoor. L: Larval sampling; As: Aspirator; BGS: BG-Sentinel trap; $\mathrm{CO}_{2}$ : Carbon dioxide; MM: Mosquito Magnet trap; HL: Human landing collection.

\begin{tabular}{|c|c|c|c|c|c|c|c|}
\hline Year & $\begin{array}{c}\text { Nr. Positive } \\
\text { companies } \\
\text { indoor (outdoor) }\end{array}$ & Municipality & Risk category & $\begin{array}{l}\text { Date first } \\
\text { detection }\end{array}$ & $\begin{array}{l}\text { Detection method (L,As, } \\
\text { BG, MM, HI) (first) }\end{array}$ & $\begin{array}{c}\text { Date last } \\
\text { finding }\end{array}$ & $\begin{array}{c}\text { Company } \\
\text { positive next } \\
\text { year? Y/N }\end{array}$ \\
\hline \multirow[t]{6}{*}{2010} & $6(3)$ & Oss* & High & $26-07-2010$ & (As), L, BGS & $23-08-2010$ & $\mathrm{Y}$ \\
\hline & & Weert $1 *$ & High & $24-08-2010$ & (L), As, MM & $27-09-2010$ & $\mathrm{Y}$ \\
\hline & & Weert 2 & High & $07-09-2010$ & (L), As, MM & 21-09-2010 & $\mathrm{Y}$ \\
\hline & & Montfoort & High & 28-09-2010 & (L), As & $05-10-2010$ & $\mathrm{~N}$ \\
\hline & & Heijningen* & High & $21-07-2010$ & (L), As, BGS & 03-09-2010 & $\mathrm{N}$ \\
\hline & & Oosterhout & High & 26-07-2010 & (As) & 06-08-2010 & $\mathrm{Y}$ \\
\hline \multirow[t]{4}{*}{2011} & $4(2)$ & Oss* & High (Cat.1) & $19-07-2011$ & (As), BGS & 29-09-2011 & $\mathrm{Y}$ \\
\hline & & Weert 1 & High (Cat.1) & $13-07-2011$ & (BGS) & $25-07-2011$ & $\mathrm{Y}$ \\
\hline & & Weert 2 & High (Cat.1) & 28-07-2011 & (L), BGS & 03-08-2011 & $\mathrm{Y}$ \\
\hline & & Oosterhout* & High (Cat.1) & $15-08-2011$ & (L, As), BGS & 05-10-2011 & $\mathrm{N}$ \\
\hline \multirow[t]{5}{*}{2012} & $5(0)$ & Oss & High (Cat.1) & $02-08-2012$ & (BGS), As & $16-08-2012$ & $\mathrm{Y}$ \\
\hline & & Weert 1 & High (Cat.1) & $10-07-2012$ & (BGS), As & $20-07-2012$ & $\mathrm{Y}$ \\
\hline & & Weert 2 & High (Cat.1) & $20-07-2012$ & $(\mathrm{BGS}+\mathrm{CO} 2)$ & $20-07-2012$ & $\mathrm{Y}$ \\
\hline & & Montfoort & High (Cat.1) & $06-08-2012$ & (BGS+CO2) & $29-08-2012$ & $\mathrm{Y}$ \\
\hline & & Heijningen & High (Cat.1) & $05-07-2012$ & (As), BGS & $25-07-2012$ & $\mathrm{~N}$ \\
\hline \multirow[t]{9}{*}{2013} & $9(7)$ & Oss* & High & $08-07-2013$ & (BGS+CO2), BGS & $18-07-2013$ & $\mathrm{Y}$ \\
\hline & & Weert $1 *$ & High & $23-07-2013$ & $(\mathrm{BGS}+\mathrm{CO} 2), \mathrm{BGS}$ & 23-07-2013 & $\mathrm{Y}$ \\
\hline & & Weert 2 & High & $23-07-2013$ & $(\mathrm{BGS}+\mathrm{CO} 2)$ & $23-07-2013$ & $\mathrm{~N}$ \\
\hline & & Montfoort* & High & $23-07-2013$ & $(\mathrm{BGS}+\mathrm{CO} 2), \mathrm{BGS}$ & $27-08-2013$ & $\mathrm{~N}$ \\
\hline & & Assen* & High & 09-08-2013 & $(\mathrm{BGS}+\mathrm{CO} 2), \mathrm{BGS}$ & 03-09-2013 & $\mathrm{Y}$ \\
\hline & & Almere & High & $22-07-2013$ & $(\mathrm{BGS}+\mathrm{CO} 2)$ & 05-08-2013 & $\mathrm{N}$ \\
\hline & & Hardenberg* & High & $02-08-2013$ & $(\mathrm{BGS}+\mathrm{CO} 2), \mathrm{L}$ & $14-08-2013$ & Y \\
\hline & & Emmeloord* & High & 01-08-2013 & (BGS+CO2), L, BGS & 24-09-2013 & $\mathrm{Y}$ \\
\hline & & Lelystad* & High & 01-08-2013 & (BGS+CO2), BGS & 21-08-2013 & $\mathrm{Y}$ \\
\hline \multirow[t]{6}{*}{2014} & $6(1)$ & Oss & High & $17-06-2014$ & $(\mathrm{BGS}+\mathrm{CO} 2)$ & $13-08-2014$ & $\mathrm{Y}$ \\
\hline & & Weert 1 & High & $17-06-2014$ & $(\mathrm{BGS}+\mathrm{CO} 2)$ & $17-06-2014$ & $\mathrm{~N}$ \\
\hline & & Assen & High & $16-06-2014$ & $(\mathrm{BGS}+\mathrm{CO2}), \mathrm{L}$ & $17-09-2014$ & $\mathrm{Y}$ \\
\hline & & Hardenberg & High & 22-09-2014 & (HL), L & $22-09-2014$ & $\mathrm{~N}$ \\
\hline & & Emmeloord* & High & $11-06-2014$ & $(\mathrm{BGS}+\mathrm{CO} 2, \mathrm{HI}), \mathrm{BGS}$ & $14-07-2014$ & $\mathrm{Y}$ \\
\hline & & Lelystad & High & $23-09-2014$ & $(\mathrm{BGS}+\mathrm{CO} 2)$ & 23-09-2014 & $\mathrm{Y}$ \\
\hline \multirow[t]{6}{*}{2015} & $6(1)$ & Oss & High & $01-07-2015$ & (BGS+CO2) & $01-07-2015$ & $\mathrm{~N}$ \\
\hline & & Montfoort & High & $13-07-2015$ & $(\mathrm{BGS}+\mathrm{CO} 2)$ & $13-07-2015$ & $\mathrm{~N}$ \\
\hline & & Assen* & High & $10-08-2015$ & $(\mathrm{BGS}+\mathrm{CO} 2), \mathrm{As}, \mathrm{HL}$ & $20-08-2015$ & $\mathrm{Y}$ \\
\hline & & Emmeloord & High & $13-07-2015$ & $(\mathrm{BGS}+\mathrm{CO} 2)$ & $11-08-2015$ & $\mathrm{Y}$ \\
\hline & & Lelystad & High & $15-07-2015$ & $(\mathrm{BGS}+\mathrm{CO} 2)$ & $15-07-2015$ & $\mathrm{Y}$ \\
\hline & & Etten-Leur & High & $13-07-2015$ & (BGS+CO2) & $13-07-2016$ & $\mathrm{Y}$ \\
\hline \multirow[t]{4}{*}{2016} & $4(1)$ & Assen & High & 24-06-2016 & (BGS+CO2) & $07-07-2016$ & not applicable \\
\hline & & Etten-Leur & High & $02-08-2016$ & $(\mathrm{BGS}+\mathrm{CO} 2)$ & $13-09-2016$ & not applicable \\
\hline & & Emmeloord* & High & $22-06-2016$ & $(\mathrm{BGS}+\mathrm{CO} 2), \mathrm{BGS}$ & $12-09-2016$ & not applicable \\
\hline & & Lelystad & High & $23-06-2016$ & $(\mathrm{BGS}+\mathrm{CO} 2)$ & 06-09-2016 & not applicable \\
\hline
\end{tabular}

Table 7. Other IMS findings in 2016 in the Netherlands from 2010-2016. $A=$ Adults; $P=$ Pupae; $L=$ Larvae.

\begin{tabular}{lll}
\hline \hline Location & Species & Total \\
\hline Weert & Ae. albopictus & $18(7 \mathrm{~A}, 8 \mathrm{~L}, 3 \mathrm{P})$ \\
Veenendaal & Ae. albopictus & $74(26 \mathrm{~A}, 44 \mathrm{~L}, 4 \mathrm{P})$ \\
\hline
\end{tabular}




\section{Discussion}

The present investigation demonstrates that eggs of IMS are repeatedly introduced at specific locations under surveillance in the Netherlands. Under favourable conditions, these eggs hatch and develop into adults. The investigation also reports, for the first time, the association of Ae. japonicus with the import of Lucky bamboo. In addition, we demonstrate that the risk categorization of used tire locations has resulted in detection of IMS exclusively at 'high risk' locations. Furthermore, occasional introductions were effectively kept under control after application of interventions against the larval and adult stages of IMS. Finally, evaluation of the proportion of samples that contain IMS after the measures specified in the covenant, suggests that the introduction of IMS at used tire and Lucky bamboo companies has stabilized and this process is not expanding.

At the Lucky bamboo greenhouses surveyed, traps for monitoring were situated at compartments in the greenhouse where Lucky bamboo was being placed in water. This water provided to the plants is required to contain a biocide effective for killing mosquito larvae. Results show that, even with the preventive measures mentioned in de Lucky Bamboo Commodities act, Ae. albopictus eggs can still hatch and develop to the adult stage in the greenhouses in spite of the biocide treatment. However, the numbers of Ae. albopictus specimens intercepted from 2010 onwards were considerably lower than our earlier results starting in 2006, in which hundreds of Ae. albopictus were captured and caused nuisance to the nursery workers (Scholte et al. 2007, Scholte et al. 2008). Additionally, in comparison with 2006-2007 (Scholte et al. 2008), there was no evidence that Ae. albopictus had escaped from the greenhouses, as oviposition traps remained negative throughout the study. One factor that may explain the observed reduction of Ae. albopictus is the use of hygroscopic gel instead of water for transporting Lucky bamboo. However, this hygroscopic gel does not entirely eliminate the risk of importing Ae. albopictus. In a study in Belgium, a live third instar larva of Ae. albopictus was found in a shipment of Lucky bamboo transported on gel, and although no water was found in the gel-bags, the gel appeared to offer a suitable medium for the short-term survival of mosquito larvae, and possibly for the eclosion of mosquito larvae from the eggs during transport (Demeulemeester et al. 2014). After the Commodities act came into effect in 2012, we initially observed few positive findings at the greenhouses in 2013 and 2014. However, results of 2015 and 2016, with new cases of IMS at Lucky bamboo companies, demonstrate that the measures need to remain accurately implemented by the Lucky bamboo importers. 
Our finding of Ae. japonicus is the first to demonstrate that this species can be associated with the import of Lucky bamboo. Furthermore, except for the possible passive transport inside horse trailers in the USA (Gaspar et al. 2012), Ae. japonicus has never been associated with trade products other than used tires. Aedes japonicus, similar to Ae. albopictus, is native to southern China (Tanaka et al. 1979) and has a similar oviposition behaviour by laying eggs in bamboo stumps (Sota et al. 1994). This may explain the presence of Ae. japonicus eggs in the Lucky bamboo imported from southern China.

The sampling strategy for detecting IMS at the used tires companies is presented here as an 'adaptive sampling strategy'. This strategy is characterized by application of adjustments in the sampling methods, frequency of inspections, and sampling areas over the course of the study, mostly informed by findings of IMS at these locations, the change in the risk category of the locations for introduction of IMS, and by the advances in IMS detecting methods. Due to the heterogeneity of the collected data obtained from an adaptive strategy, a descriptive analysis has been performed for most of the data collected in the study. In 2010, first IMS findings were recorded using only larval sampling or aspirators (see Table 6). In 2011, first findings were also obtained using the same methodology, but for the first time a BGS trap detected an IMS at a location where larval sampling or adult collections remained negative. This underscores the added value of the deployment of traps in our surveillance strategy. Furthermore, in 2011, in all positive locations, at least one positive sample was obtained using BGS traps. In 2012, the situation changed when four of five positive locations were first detected using BGS traps rather than with larval sampling or aspirators. Based on the results from the first three surveillance years (2010-2012), it was decided to place one BGS trap (with $\mathrm{CO}_{2}$ as added stimulus) in all high risk locations from 2013 onwards. In several studies in Europe (Werner et al. 2012, Becker et al. 2013, Montarsi et al. 2015, Venturi et al. 2017), the BGS traps have demonstrated effective for capturing IMS. Although the use of larval sampling and aspirators can be effective for detecting IMS, the use of traps and especially the addition of $\mathrm{CO}_{2}$ is considered advantageous because (1) traps containing lures attract and capture IMS throughout the day in comparison with fortnightly larval sampling inspections, (2) the effectivity of the sampling of adult IMS using aspirators strongly depends on the choice of days with good weather for sampling (e.g. no rain, adequate temperature, no wind), (3) traps can detect IMS adults emerging from tire stacks that are not accessible for adequate larval sampling, and (4) the addition of $\mathrm{CO}_{2}$ increases the chance of detecting IMS that are less attracted to the BG-lure (such as Ae. japonicus). 
From 2010 to 2016, the earliest detection of an adult IMS was 11th June 2014 in Emmeloord. At other locations (e.g. Assen, Weert and Oss), IMS were also found later that same month. These early findings may be related to the exceptionally warm weather in the spring of 2014, as 2014 was recorded as the hottest year in three centuries (https:/www.knmi.nl/kennis-endatacentrum/achtergrond/2014-record-warm). Interestingly, the finding was reported by a company worker that suffered bites of one aggressive Ae. albopictus female at the company canteen. Samples of traps that were running at this company were also positive for $A e$. albopictus $(\mathrm{n}=6)$. In other words, passive detection by company workers and active sampling using traps seemed equally sensitive in detecting Ae. albopictus. This was not the case in Hardenberg. Here, a picture of a mosquito taken from this location was evaluated and confirmed as Ae. albopictus. It was then decided to intensively inspect the company property for the presence of larvae and adults, and placing extra BGS traps. However, Ae. albopictus was not found in the $\mathrm{CO}_{2}$-supplemented BGS-trap at the company, nor at traps placed elsewhere, nor during the larval sampling. Interestingly, during the intensive inspection at this company, one larva of Ae. japonicus was found in the tires stored at the company. This confirms, for the first time, the import of this species with imported tires in the Netherlands. Until 2014, Ae. japonicus had been only found in the municipality of Lelystad, where this IMS has established populations. The finding of Ae. japonicus in 2013 during the used tires surveillance in Lelystad (see Table 5 and Table 6) was recorded using a BGS-trap outside the company (Ibañez-Justicia et al. 2014). The finding of a larva in tires suggests that populations in Lelystad could have built up from successive introductions of infested tires at the used tires location located at the municipality, and before the surveillance activities were introduced in used tire companies in 2010. Later in 2015, another finding of an adult female of Ae. japonicus in a BGS-trap inside a used tire company in Oss further confirmed the import of this species with used tires.

From 2011 to 2016 (2010 not considered), 25 out of 34 introductions occurred at companies that were found positive the year before, suggesting that at these locations, new sets of used tires containing eggs of IMS were introduced, leading to new findings the following year. It is not known whether the IMS successfully overwintered at these companies during the sampling years, or if it was reintroduced each year through transport of infested tires. It is, therefore, recommended to study the lower temperature thresholds that 1) could limit the ability of Ae. albopictus to establish permanent populations over multiple years and 2) prevent population buildup during the warmer periods of the year. 
Two mosquito species found during the surveillance were introduced with used tires from North America (Scholte et al. 2009, Scholte et al. 2010a): Ae. aegypti and Ae. atropalpus. The first was no longer detected after the first finding and after subsequent mosquito control applied in 2010. Similarly, Ae. atropalpus was found in 2010 and 2011, and following mosquito control it was no longer detected at any of the sites. In comparison with the number of used tires imported from areas in southern Europe where Ae. albopictus has established, the number of used tire shipments from North America is considered small.

Data on used tire volumes imported into the Netherlands show that, except in 2014, the annual numbers of used tires imported are higher than in our first year of study (2010, see Figure 2). In 2015 and 2016 in particular, the number of imported used tires was double the number of the years before. After the application of the preventive measures laid down in the covenant, we expected a reduction in the risk for introduction of IMS at the used tire companies. Considering that the covenant was signed in June 2013, the specified measures could influence the results from 2014 onwards. Considering the proportion of positive samples during the study period, an increase in this proportion could be expected (more imported tires, more possibilities for introduction of infested tires from other countries). Rather, the proportion of positive samples remained significantly lower than the proportion in 2013, prior to the implementation of the covenant. Whether this is an effect that can be ascribed to the application of the covenant remains unknown. Although the application of the preventive measures has not completely eliminated the risk of introduction, it seems to have stabilized the situation and thus kept the introductions under control.

Overall, it can be considered that the yearly results of categorizing locations for used tires has resulted in detection of IMS in the country. Results show that all findings have been recorded at locations that were categorized as 'high' risk in our risk analysis. Sam pling at 'm edium ' or 'low' risk locations always rem ained negative for presence of IMS. In conclusion, data on introductions of IMS provided by surveillance are needed to evaluate the risk associated with potential introduction locations, as well as to assess the effectiveness of surveillance and control measures. In the Netherlands, until 2018, IMS control was a task of the local government and the implementation of preventive measures by tire traders could not be enforced by law under the covenant. Change of this legislation in 2018 will make the Minister of Health, Welfare and Sport, instead of the municipalities, responsible for both preventive measures and control, and it will also allow the NVWA to enforce implementation of these measures. However, as experienced in 2016, new IMS introductions can occur giving the 
invasive species the opportunity to proliferate and establish populations at locations outside our risk-based surveillance locations. Prompt communication on the presence of IMS to the NVWA is crucial because of the possible increase of these introductions facilitated by the ability of IMS to travel with human passive transport (e.g. cars, trucks, trains), and the presence of established populations in neighbouring countries.

\section{Acknowledgments}

We thank Mr. Anton van der Sommen for the critical review of the manuscript. We also thank Mr. Roel Potting for providing information of import of Lucky bamboo plants, and Mr. Arie Verhoef for providing information of used tire companies used for the categorisation. NVWA colleagues are especially thanked for their assistance in the lab and in the field. 



\title{
Chapter 7
}

\section{The first detected airline introductions of yellow fever mosquitoes (Aedes aegypti) to Europe, at Schiphol International airport, the Netherlands}

\author{
Adolfo Ibáñez-Justicia ${ }^{1}$, Andrea Gloria-Soria ${ }^{2}$, Wietse den $\operatorname{Hartog}^{1}$, Marian Dik ${ }^{1}$, Frans \\ Jacobs $^{1}$ and Arjan Stroo ${ }^{1}$
}

1. Centre for Monitoring of Vectors (CMV), Netherlands Food and Consumer Product Safety Authority (NVWA), Wageningen, The Netherlands

2. Department of Ecology and Evolutionary Biology, Yale University, New Haven, USA

Published in Parasites \& Vectors (2017) 10(1):603, doi: 10.1186/s13071-017-2555-0. 


\section{Abstract}

Background: Air-borne introduction of exotic mosquitoes to Schiphol airport in the Netherlands has been considered plausible based upon findings of mosquitoes in aircraft cabins during 2008, 2010 and 2011. Beginning in 2013, surveillance efforts at Schiphol had focused on promptly detecting accidental introductions at the airport facilities in order to quickly react and avoid temporary proliferation or establishment of mosquito populations, identify the origin of the introductions, and avoid potential transmission of vector-borne diseases. Methods: BG-Mosquitaire mosquito traps were set at the most likely locations for arrival of the invasive Aedes mosquitoes as part of the mosquito monitoring program at Schiphol airport. Samples were collected bi-weekly. Upon detection of exotic specimens, information about the origin of the flights arriving to the particular location at the airport where specimens were captured was requested from airport authorities. The GIS tool Intersect was then used to identify airports of origin common to positive trapping locations during the specific trapping period. Captured Aedes aegypti mosquitoes were subsequently genotyped at 12 highly polymorphic microsatellite markers and compared to a reference database of 79 populations around the world to further narrow down their location of origin. Results: In 2016, six adult yellow fever mosquitoes were captured indoors and outdoors at the airport of Schiphol in the Netherlands confirming, for the first time, air-borne transport of this mosquito vector species into Europe. Mosquitoes were captured during three time periods: June, September and October. Containers carried by aircrafts are considered the most likely pathway for this introduction. GIS analysis and genetic assignment tests on these mosquitoes point to North America or the Middle East as possible origins, but the small sample size prevents us from reliably identifying the geographic origin of this introduction.

Conclusions: The arrival of Ae. aegypti mosquitoes to Schiphol airport from flights arriving from overseas, demonstrates the potential risk of international flights to public health as carriers of arthropod vectors of disease. The results strongly suggest that disinsection of containers and their storage compartments inside the aircrafts could contribute to preventing future introductions of mosquito vectors. Invasive mosquito species introduced by aircrafts from overseas could become seasonally established during the warmer months in Europe, or permanently in certain climatically suitable areas for the species, with major consequences for human health.

Keywords: Aedes aegypti, airport introduction, mosquito surveillance, population genetics, microsatellite markers 


\section{Background}

Global spread of the yellow fever mosquito, Aedes aegypti, has historically been related to human trade and transport. In this way, the species has successfully colonized suitable locations in countries of all continents, except Antarctica. The northernmost documented occurrences in Europe (i.e. Bordeaux and Saint Nazaire, France; Swansea and Southampton, UK) arose from introductions via ships, and no evidence was found on the spread or establishment of the species at these places (Schaffner and Mathis 2014). Historically, the species also displayed a much larger distribution in Europe compared to its current one (Schaffner and Mathis 2014).

In the 19th century, Ae. aegypti-driven outbreaks of yellow fever took place in port cities in northerly areas of Europe (e.g. Saint-Nazaire, France; and Swansea, UK). The 20th century also witnessed one of the largest outbreaks of dengue recorded globally in Athens and neighboring areas of Greece in 1927-1928 (Schaffner and Mathis 2014). Urban yellow fever was eliminated from many countries after the Second World War, by energetic campaigns to eliminate Ae. aegypti breeding sites through application of the pesticide dichlorodiphenyltrichloroethane (DDT) to infested containers and their surroundings (Reiter 2010).

In more recent times, globalization of trade and travel has facilitated the geographical spread of vectors and vector-borne diseases (Gratz 1999, Ahmed et al. 2009), with air travel playing a major role in long-distance dispersal (Gezairy 2003, Tatem et al. 2006). In areas of high mosquito densities, mosquitoes can follow their human hosts unnoticed and enter aircrafts at the airports (Gratz et al. 2000). These aircrafts can then transfer mosquitoes from one location to another relatively rapidly, thus increasing their chance of mosquitoes surviving the trip and reaching the destination. Upon arrival in another country, mosquitoes may colonize new areas or infect people locally (Gratz et al. 2000, Bataille et al. 2009). For example, a mosquito which alighted from Bali with a military aircraft was considered the most likely source of a case of "airport dengue" in Australia in 2010 (Whelan et al. 2012).

In 2008, the Netherlands reported live mosquitoes on a flight from Dar es Salaam, Tanzania, to Schiphol airport in Amsterdam, with several passengers complaining of being bitten on board. Mosquitoes collected by the flight attendants were identified as Culex quinquefasciatus Say (Scholte et al. 2010b). A follow-up study in 2010 and 2011 focusing on answering basic questions as to the mosquito species, frequency, and number of mosquitoes 
on board of aircrafts that land at Schiphol airport, showed that exotic mosquito vectors of diseases (Cx. quinquefasciatus, Culex antennatus and Aedes mcintoshi) were transported on 10 of the 38 aircrafts inspected (Scholte et al. 2014). Air-borne movement of mosquitoes has thus proven to be possible based upon these findings. Given the high volume of air transport movements per year between Schiphol and vector-borne disease endemic regions in the world, establishment of mosquito vectors after introduction via aircraft should be considered a potential health risk.

Since 2013, surveillance efforts at Schiphol have focused on promptly detecting accidental introductions at the airport facilities, with the goal of eliciting a quick reaction to avoid temporary proliferation, identifying the origin of the introductions, and avoiding transmission of vector-borne diseases. Here we describe the methodology used for surveillance of mosquitoes at the airport of Schiphol and report the findings of yellow fever mosquitoes ( $A e$. aegypti) at the airport facilities in 2016. We also detail the vector-control response that followed these events. Efforts to identify the origin of the introductions combined data on flight departure locations provided by the airport authorities, the use of suitability maps, and genetic assignment tests of the specimens captured to a reference database of world populations based on 12 highly polymorphic microsatellite markers (Gloria-Soria et al. 2016). Identifying the origin of introduced invasive mosquito species is important to aid public health efforts to prevent the introduction and spread of these vectors of humandiseases.

\section{Methods}

\section{Mosquito collection and identification}

Since 2013, mosquito monitoring at Schiphol airport has mainly consisted of placing mosquito traps at the most likely locations for the arrival of invasive Aedes mosquitoes. Examples of such locations are: platforms of arrival gates with high volumes of international flights landings from overseas, platforms of gates for European flights arriving from areas where invasive mosquitoes are present (e.g. Italy, southern France), indoor locations where suitcases are unloaded, temporary storage locations for arriving cargo, imported fruits and vegetables, or animals.

The traps used at Schiphol airport are BG Mosquitaire traps (Biogents AG, Regensburg, Germany). These traps have been specifically developed for capturing Aedes mosquitoes (Ae. 
albopictus, Ae. aegypti and related species) and use a patented mix of artificial skin emanations (BG-Sweetscent), in combination with air convection and light-and-dark contrasts. Carbon dioxide is not used at the airport. Lure used in the traps has proven to be efficient for capturing adult Ae. albopictus and Ae. aegypti (both males and females) in the field (de Azara et al. 2013, Degener et al. 2014, Unlu and Farajollahi 2014). Traps were continuously operated (24/7) and placed indoors and outdoors in shaded, wind-protected moist areas.

Mosquito samples were collected bi-weekly. Trapping nets from the BG trap with collected mosquitoes and a data form were sent together inside a sealed plastic bag to the laboratory of the National Reference Centre from the Food and Consumer Product Safety Authority (NVWA/NRC) for morphological identification. All data from each sampling location were submitted into VecBase (database of Centre for Monitoring of Vectors - CMV). In the laboratory, mosquitoes were counted and morphologically identified by specialists using the keys of Schaffner et al. (2001) and Becker et al. (2010). Exotic Aedes species diagnosis was validated via a real-time PCR assay within the NVWA/NRC, specially developed for the CMV surveillance for the detection of Ae. albopictus, Ae. aegypti, Ae. atropalpus and Ae. japonicus (van de Vossenberg et al. 2015). In 2016, a maximum of ten mosquito traps were continuously functioning at the airport and routinely checked every two weeks. The action plan in place to respond to an invasive mosquito species introduction (IMS) is as follows: (i) Both the number of traps and their monitoring frequency are increased 2-fold at the airport to check for additional locations hosting these mosquitoes. (ii) Oviposition traps, black plastic containers filled with hay infusion and a piece of Styrofoam serving as the oviposition substrate, are deployed in the surroundings of the first findings to detect a possible establishment of the introduced species. At the airport, the top of the oviposition traps is covered with stainless steel bird netting to prevent the Styrofoam from been blown away by the aircraft engines. (iii) The search for IMS larvae is performed in potential breeding sites at the airport, prior to implementation of mosquito control interventions. Upon detection of $A e$. aegypti in 2016, this plan was implemented as described.

\section{Flight origin data}

Information about the origin of the flights arriving at each particular location (or gate) during the trapping period when exotic specimens were captured was requested from the airport 
authorities. A file was provided with the following information: date, flight number, estimated arrival time, origin (IATA Code), arrival platform and luggage handling area. The file was compared with the database Openflights.org (2016) to find the name of the airport of origin, the city name, country, and geographical coordinates. Coordinates of the selected airports were input into ArcGIS 10.3 to generate a map indicating the candidate airports for bringing the exotic specimens during each positive trapping period. Using the intersect tool of ArcGIS we identified the airport origins common to several trapping location inputs during the desired period (that is, they intersect). Combining this map with suitability maps for $A e$. aegypti mosquitos based on (Kraemer et al. 2015a), we generated a new map identifying the airports around the world situated in areas were the target species could be present. Airports were considered as "non-suspected" of introducing the species when the species was absent and/or the probability of a mosquito population in the area was considered low.

\section{DNA extraction and genotyping}

Total genomic DNA from Ae. aegypti adult legs was extracted using either High Pure PCR Template Preparation Kit (Roche, Basel, Switzerland), or the DNeasy Blood and Tissue kit (Qiagen, Venlo, the Netherlands), according to the manufacturer's instructions, with an additional RNAse A (Qiagen) step. Samples were stored at $-20{ }^{\circ} \mathrm{C}$ until further analysis. Individual mosquito material was genotyped as described in (Brown et al. 2011b). The microsatellite loci analyzed were: A1, B2, B3, A9 (tri-nucleotide repeats) and AC2, CT2, AG2, AC4, AC1, AC5, AG1 and AG4 (di-nucleotide repeats) (Slotman et al. 2007). Polymerase chain reactions were conducted as $10 \mu \mathrm{l}$ reactions using the Type-it Microsatellite PCR Master Mix (Qiagen), $25 \mathrm{nM}$ of each forward primer, $250 \mathrm{nM}$ of each reverse primer, and $500 \mathrm{nM}$ of a fluorescently labeled M13 primer. Thermocycler conditions were: $94{ }^{\circ} \mathrm{C}$ for $10 \mathrm{~min}, 35 \times\left(94^{\circ} \mathrm{C}\right.$ for $30 \mathrm{~s}, 54{ }^{\circ} \mathrm{C}$ for $30 \mathrm{~s}, 72^{\circ} \mathrm{C}$ for $\left.30 \mathrm{~s}\right)$, and $72{ }^{\circ} \mathrm{C}$ for 5 min. The resulting products were processed for fragment analysis at the DNA Analysis Facility at Science Hill at Yale University, USA, using GS 500 Rox internal size standard (Applied Biosystems, Foster City, USA). Microsatellite alleles were scored using GeneMapper v4.0 (Applied Biosystems). Raw allele frequencies of the reference panel are available at VectorBase.org. Raw microsatellite allele calls for the mosquitoes captured at Schiphol can be found in Additional file 1: Table S1. 


\section{Population genetic analysis}

Genetic assignment tests were performed in GENECLASS2 (Piry et al. 2004), using the Bayesian criteria for likelihood estimation with a threshold value of 0.05 (Rannala and Mountain 1997). The reference population panel included the 79 populations $(n=3632$ individuals) from the six continents around the world where Ae. aegypti is present (GloriaSoria et al. 2016), and includes members from the subspecies Ae. aegypti aegypti as well as Ae. aegypti formosus. To estimate the accuracy of this individual assignment method using our reference panel, we selected 100 random individuals from the same panel (with at least one representative of each population) and re-assigned them to the reference panel without replacement (as if they were newly acquired samples). During this exercise, $76.2 \%$ of the individuals were correctly assigned back to their population of origin, and for $93 \%$ of the samples the correct population of origin was ranked among the top 5 assignment scores. It is worth noting that the individuals that were not accurately assigned to their original population were assigned to a population with a similar genetic signature and in close geographic proximity (e.g. Conch Key, FL rather than Miami, FL, USA). In addition to the individual assignm ent tests, the Netherlands' individuals collectedin the same period at the airport were also treated as belonging to the same population and a group genetic assignment was performed following the same criteria.

\section{Results}

\section{Mosquito collection}

The BG traps monitored every two weeks at the airport, only captured indigenous mosquitoes (at low abundance) in 2016 until June. These included: Anopheles maculipennis (sensu lato) $(n=17)$, Culiseta annulata $(n=15)$, and Culex pipiens/torrentium $(n=1316)$. Three adult Ae. aegypti were captured at two airport locations in June 2016 (see Table 1). One specimen was captured in a trap situated outdoors (Platform E), next to the arrival platform of intercontinental flights, and the other two specimens were captured indoors next to a mechanical unloading system named MUM (MUM 2016), an innovative mechanical system that automatically unloads baggage/luggage containers, the type of containers used by airlines to transport suitcases. All three specimens were males, and all three were identified as $A e$. aegypti using real-time PCR (van de Vossenberg et al. 2015). As a result of increasing the number of traps and trapping locations upon the first finding, one female Ae. aegypti was 
identified on September 13th, captured outdoors in a BG-trap at a different gate (Platform G), and on September 29th a second adult Ae. aegypti female was captured at a BG-trap at the MUM. The third and last finding (one female) was recorded in an indoor BG-trap placed in a baggage handling area of the airport (between platforms $F$ and $G$ ) at the end of October (Table 1). The finding at this location was a consequence of the intensive surveillance implemented as part of the vector-control response. In total, six Ae. aegypti specimens were intercepted in 2016, both indoors and outdoors. These events can be considered as a minimum of a single introduction that persisted over the months, or as many introductions as the number of individual mosquitoes detected $(n=6)$. However, based on the distribution of the trapping periods and because none of the 27 infusion-baited oviposition traps placed in the surroundings of the first finding in June (Platform E, and MUM) were positive for exotic mosquitoes prior to and after the detection, we suspect three detected introduction events to Schiphol, in June (3 specimens), in September ( 2 specimens) and in October (1 specimen).

Table 1. Aedes aegypti findings in 2016 at Schiphol airport, the Netherlands. ${ }^{a}$ Dates are reported in a daymonth-year format

\begin{tabular}{lllll}
\hline $\begin{array}{l}\text { Date of } \\
\text { placing }\end{array}$ & $\begin{array}{l}\text { Date of } \\
\text { sampling }\end{array}$ & Location & No./Sex & Sample ID \\
\hline $24-05-2016$ & $09-06-2016$ & Platform E (outdoors) & $1 /$ Male & Neth16_802 \\
24-05-2016 & $09-06-2016$ & Mechanical unloading system (indoors) & $1 /$ Male & Neth16_845 \\
09-06-2016 & $15-06-2016$ & Mechanical unloading system (indoors) & $1 /$ Male & Neth16_853 \\
29-08-2016 & $12-09-2016$ & Platform G (outdoors) & $1 /$ Female & Neth16_6637071 \\
12-09-2016 & $26-09-2016$ & Mechanical unloading system (indoors) & $1 /$ Female & Neth16_6813416-1-85 \\
$17-10-2016$ & $31-10-2016$ & Baggage handling (indoors) & $1 /$ Female & Neth16_4388663 \\
\hline
\end{tabular}

Larval sampling for IMS in all potential breeding sites at the airport was put in place prior to the mosquito control response. A total of 16 samples were collected containing exclusively indigenous mosquitoes of the genus Culex (pupae $(n=40)$, larvae $(n=946))$. A population of autogenous $C x$. pipiens biotype molestus breeds in stagnant water in the basements of Schiphol airport. Samples were taken at those breeding sites, but only this species was present.

\section{Tracing the origin of aircraft flights}

During the trapping-period when Ae. aegypti specimens were captured, flights from 206 airports around the globe landed at Schiphol airport (Table 2). 
Assuming that all Ae. aegypti specimens intercepted at the airport in June (indoors in the MUM, and outdoors) have the same origin, 36 possible airport origins were identified after combining the origin data from flights arriving at both sites during the catching period. Among others, flights from airports in North America, Middle East and Asia arrived to Schiphol during the findings in June at both locations (see Fig. 1).

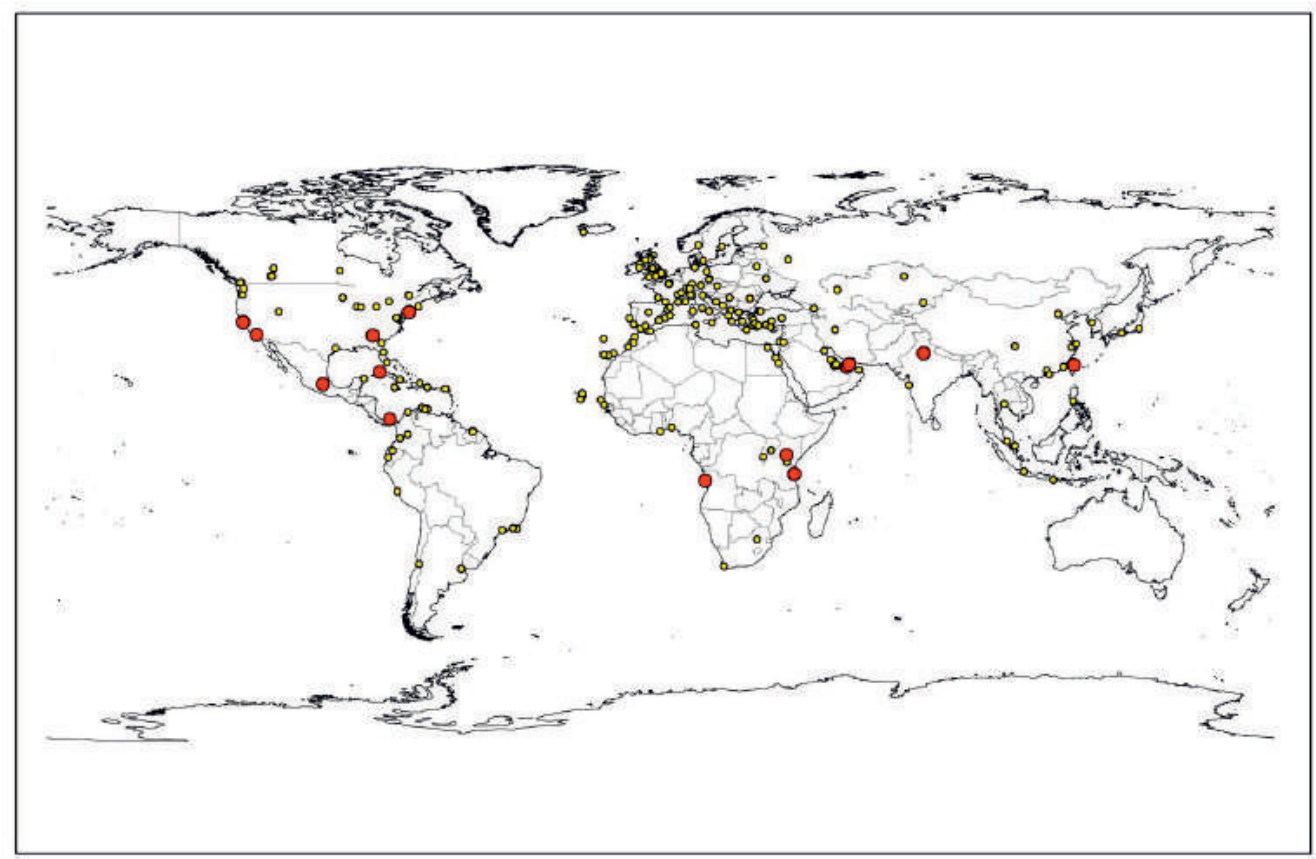

Fig. 1 Origin of flights arriving at locations where Ae. aegypti has been found in Schiphol airport. All origins in June, September and October $(n=206$; yellow dots). Intersect (common) origins June, September and October $(n=14$; red dots)

Intersecting the data of airport origins from flights that arrived to the locations where the specimens were trapped in September (outdoors and indoors), we identified 26 potential origins that included airports in North America (including Florida), Middle East (Saudi Arabia) and Asia (see Fig. 1). 
Table 2. Number of airport origins identified arriving at the trapping locations. ${ }^{\mathrm{a}}$ Dates are reported in a day-month-year format.

\begin{tabular}{cclc}
\hline $\begin{array}{r}\text { Date of } \\
\text { placing }^{\mathbf{a}}\end{array}$ & $\begin{array}{c}\text { Date of } \\
\text { sampling }^{\mathbf{a}}\end{array}$ & Location & $\begin{array}{c}\text { No. of airport origins } \\
\text { identified arriving at the } \\
\text { trapping locations }\end{array}$ \\
\hline $24-05-2016$ & $09-06-2016$ & Platform E (outdoors) & 65 \\
$24-05-2016$ & $15-06-2016$ & Mechanical unloading system (indoors) & 58 \\
$24-05-2016$ & $15-06-2016$ & Intersect (June) & 36 \\
$29-08-2016$ & $12-09-2016$ & Platform G (outdoors) & 79 \\
$12-09-2016$ & $26-09-2016$ & Mechanical unloading system (indoors) & 61 \\
$29-08-2016$ & $26-09-2016$ & Intersect (September) & 26 \\
$17-10-2016$ & $31-10-2016$ & Baggage handling (indoors) & 179 \\
$24-05-2016$ & $31-10-2016$ & Intersect (June-September-October) & 14 \\
$24-05-2016$ & $31-10-2016$ & All origins merged & 206 \\
\hline
\end{tabular}

Data provided by the Schiphol authorities for the October findings provided 179 possible origins because this baggage location processes multiple arrivals. At this location, several baggage-handling companies work with a variety of container types or unload directly from the cargo space of the airliner to lorries in open air.

Assuming that all Ae. aegypti specimens intercepted at the airport originated from the same introduction, or that all specimens captured were carried in aircrafts proceeding from the same airport origin, we have identified 14 airports that originated flights arriving at all sites during the three catching periods. These airports are located in: Angola, Tanzania, Kenya, Panama, Mexico, Cuba, United States of America, United Arab Emirates, Taiwan and India (see Fig. 1).

\section{Population genetic analysis}

The results from the genetic assignment analysis performed with GeneClass2 (Piry et al. 2004) on the Ae. aegypti specimens sent to Yale University to identify the likely origin of the introduction in Schiphol are shown in Table 3. Except for the last sample collected in October, all of the other five samples were successfully genotyped.

When each individual was treated as an independent introduction, the analysis identified four potential sources, two from Asia (Saudi Arabia and Philippines) and two from North America (Miami and Palm Beach in Florida, USA) (see Table 3). When individuals were grouped by the month in which they were captured and treated as two introductions: June (3 individuals) and September (2 individuals), the analysis identified two potential sources, Miami and Saudi Arabia with a $99.95 \%$ and $95.47 \%$ assignment probability, respectively (see Table 3). 
However, when the individuals are all considered part of the same population, either as a result of the introduction of multiple individuals at once, a population already established at the airport, or multiple introductions carried by different aircrafts originating at the same foreign airport, the highest assignment score indicates Saudi Arabia as the most likely source, followed by the Philippines (Table 3 ).

Table 3. Genetic assignment results from the Aedes aegypti specimens found at Schiphol airport, the Netherlands. ${ }^{\mathrm{a} D}$ Dates are reported in a day-month-year format. ${ }^{\mathrm{b}}$ Individual frequency assignments; populations assigned the highest assignment score $(0-100 \%)$. ${ }^{\circ}$ Group assignment test; populations assigned the highest assignment score [0-100\%]. The three locations with the highest assignment score are shown. Abbreviation: na, not available.

\begin{tabular}{|c|c|c|c|c|}
\hline Period $^{\mathrm{a}}$ & Sample ID & Individual assignment $^{b}$ & Group $A$ assignment $^{\mathrm{c}}$ & $\begin{array}{l}\text { Group B } \\
\text { assignment }^{\mathrm{c}}\end{array}$ \\
\hline \multirow{3}{*}{$\begin{array}{l}24-05-2016 \\
16-06-2016\end{array}$} & Neth16_802 & Saudi Arabia $(87.350 \%)$ & Miami, FL, USA & \multirow{5}{*}{$\begin{array}{l}\text { Saudi Arabia } \\
(79.85 \%) \text {; the } \\
\text { Philippines } \\
(19.33 \%) \text {; New } \\
\text { Orleans, USA } \\
(0.78 \%)\end{array}$} \\
\hline & Neth16_845 & Miami $(68.590 \%)$ & (99.95\%); Rio, FL, & \\
\hline & Neth16_853 & Palm Beach (32.990\%) & $\begin{array}{l}\text { USA }(0.04 \%) \text {; } \\
\text { VacaKeys,FL, USA } \\
(0.01 \%)\end{array}$ & \\
\hline \multirow{2}{*}{$\begin{array}{l}29-08-2016 \\
26-09-2016\end{array}$} & Neth16_6637071 & The Philippines (53.557\%) & \multirow{2}{*}{$\begin{array}{l}\text { Saudi Arabia (95.47\%); } \\
\text { Philippines (4.40\%); } \\
\text { New Orleans, USA } \\
(0.07 \%)\end{array}$} & \\
\hline & Neth16_6813416-1-85 & Saudi Arabia (82.855\%) & & \\
\hline $\begin{array}{l}17-10-2016 \\
31-10-2016\end{array}$ & Neth16_4388663 & na & na & na \\
\hline
\end{tabular}

\section{Discussion}

\section{Detection of first Ae. aegypti in Schiphol airport}

The spread of Ae. aegypti around the world is most commonly associated with the transport of artificial containers infested by eggs or immatures (Eisen and Moore 2013). However, the current study focuses on the movement of yet another developmental stage of great epidemiological importance: the adult mosquito. Schiphol airport in the Netherlands intercepted six adult yellow fever mosquitoes in 2016, confirming the possibility of air-borne introduction of this mosquito vector species to Europe, and the first finding of this species within a European airport facility. Aircraft arriving to Schiphol airport from areas hosting a large population of Ae. aegypti could introduce mosquitoes rapidly from abroad, thus increasing their chance of survival in receptive areas (Gratz et al. 2000). Aedes aegypti mosquitoes intercepted in June and in September at Schiphol airport were captured in outdoor and indoor traps. The trapping location choices, devices and lures used to detect this IMS (BG-mosquitaire with BG-Lure) proved to be effective for both male and female Ae. aegypti. Capture of male specimens is not unexpected, since Ae. aegypti males mate near their host 
(Hartberg 1971). The intercepted mosquitoes presumably traveled from North America or Asia, thus surviving a minimum of seven hours inside an aircraft during the flight. These mosquitoes then left the aircraft (or the containers) when doors opened and eventually flew to the traps. Finding of the specimens indoors, next to locations where sealed containers carrying personal baggage are opened for first time after arrival from other countries, suggests that Ae. aegypti could have either followed a cargo-handler inside the container and was trapped inside, or that the mosquito was attracted by the human odours emanating from the suitcases/baggage at the airport of origin. If the latter were true, cargo planes containing goods would probably be less attractive to this species than planes transporting humans and their personal belongings.

\section{Public health implications}

Introductions of Ae. aegypti from endemic countries at this busy airport imply a major public health risk. The presence on board of mosquitoes from areas with endemic arthropodtransmitted diseases (e.g. malaria endemic areas) put passengers at immediate health risk through biting during the flight (Mangili and Gendreau 2005). Upon arrival in another country, infected mosquitoes could transmit the pathogen locally in and around the airport, as is the case of "airport m alaria" (Gratz et al. 2000, Bataille et al. 2009). Aedes aegypti transmits dengue virus, yellow fever virus, chikungunya virus and Zika virus, among other arboviruses (Marchette et al. 1969, Fontenille et al. 1997, de Lamballerie et al. 2008, Ramchurn et al. 2009). Because of the short interval of time required for completing the journey, air transport has been associated with a higher risk of introducing a live infected mosquito compared to, for example, sea or road transport (Whelan et al. 2012).

Suitability maps suggest that the climate in the Netherlands is not favorable for the survival and establishment of Ae. aegypti (Kraemer et al. 2015a). Unlike Ae. albopictus eggs, Ae. aegypti eggs cannot diapause, thus could not survive the outdoor Dutch winter conditions. Nevertheless, evidence suggests that Ae. aegypti could overwinter within human microhabitats in regions where the low temperatures would otherwise not allow them to survive (Lima et al. 2016). Therefore, constant vector surveillance to prevent the introduction of invasive mosquito species beyond its native range is imperative to prevent transmission of pathogens transmitted by these mosquitoes. 


\section{Vector control response}

Aedes aegypti establishment strongly depends on the availability of artificial water-holding containers for oviposition and larval development. The presence of water-holding containers at Schiphol airport, prior to the Ae. aegypti findings, was considered low. However, as part of the vector-control response, operators of Schiphol facility services applied hot water pressure cleaning machines to the drainage water system and rain gutters at all terminals in order to eliminate any larvae/pupae that could be present and avoid water stagnation. Water containers found (stagnant water) were sampled for mosquito immatures and later preventively treated or eliminated. Wells with stagnant water, holes, and open PVC cable tubes containing water were also closed with insulation foam or concrete. Paraffin oil was added to stagnant water locations inside the buildings at the underground level. These strategies allow us to rule out an established population of this mosquito in the airport.

\section{Origin of Ae. aegypti introduced to Schiphol}

Genetic assignment tests allow us to broadly identify the region of origin of Ae. aegypti intercepted at Schiphol. Similar analysis was performed on Ae. aegypti mosquitoes imported into the Netherlands in 2010, successfully tracking the origin of these mosquitoes to a tire shipment from Miami, Florida, USA (Brown et al. 2011a). However, in this introduction at the airport, the specific location could not be further narrowed down due to the small number of specimens collected and the uncertainty of whether they belonged to the same source population or multiple sources. Furthermore, the ability of the genetic tests to identify the source of the introduction is limited by the representation of the source area in the genetic reference dataset. Nevertheless, populations of Ae. aegypti display a strong hierarchical geographic signature (Scholte et al. 2014). Thus, even if the source population was absent from the dataset, the genetic assignment test will point to a geographic population represented in the dataset that shares a genetic signature with the source location, a geographically close location. One should also bear in mind that a portion of containers arriving to the airport could have been transferred from other routes at one or multiple airports prior to the final arrival and information of prior transfers was not available from the airport authorities.

Since the mosquitoes were not trapped inside the aircrafts but at the airport facilities, one should consider the possibility that Ae. aegypti was already present on the ground. The mosquitoes captured could be remnants of the earlier introduction to the Netherlands in 2010 
(Brown et al. 2011a). However, we consider this unlikely because the Ae. aegypti individuals intercepted in 2010 were breeding in tire yards, that were treated with adulticides and larvicides at the time and the follow up on these vector control measures determined that the elimination was successful (Scholte et al. 2010a). The tire yard and the airport are separated by more than $70 \mathrm{~km}$ and there has not been any report of Ae. aegypti in the time interval between the 2010 and 2016 .

\section{Future recommendations}

Identifying the origin of introduced invasive mosquito species is relevant to aid public health efforts to prevent the introduction and spread of disease vectors. In the case of Schiphol airport, upon knowing the exact origin of the flights transporting the species and the companies involved, the following measures could be put in place: (i) require the involved airline agencies to execute special disinsection measures at the airport of origin inside the containers; (ii) inform airport authorities so they can handle the containers from infested origins in quarantine areas designed to prevent possible introductions; (iii) inform the European invasive mosquito surveillance teams about the findings, especially from European regions with a climate suitable for the establishment of invasive mosquitoes in Europe (Turkey, Spain, Italy, etc.).

The current recommendations of the WHO for aircraft disinsection with insecticides (WHO 1995), include specifications for aerosols and approved insecticidal formulations, and include three preferred methods: disinsection before take-off ("blocks-away" disinsection), disinsection top-of-descent (spraying of the cabin is done immediately after the aircraft started its descent to destination), and disinsection with residual insecticide. Disinsection on the ground ("on-arrival" disinsection) may be retained as an acceptable back-up method if an aircraft, coming from areas of threat, has not been adequately disinsected by any of the preferred methods (WHO 1995). The finding of specimens at the baggage handling area suggest that disinsection did not take place inside the transported containers, nor within the baggage compartment of the aircraft. Alternatively, it may have been applied and was not efficient, and therefore could lead to subsequent introductions of invasive mosquitoes. Based upon the evidence presented above, the authors consider that for a thorough disinsection, it is of crucial importance to include the containers and the container compartments of the aircrafts. 
Additionally, sample degradation during trapping or handling of specimens, or a limited amount of biological material for genetic analysis, can compromise subsequent genetic work. Thus, if the genetic analysis were to be incorporated as part of the vector control program, it is necessary to establish protocols aimed at maximizing the information obtained from the specimens collected, and to promote close communication between the vector control agencies and the genetic test facilities. For example, the number of mosquito traps (lured and ovitraps) could be increased at the airport to increase the detection power and subsequently, obtain more information about the introductions and provide more material for the genetic analysis. It is also recommended to increase the sampling frequency to be performed weekly instead of fortnightly at the airport. Additionally, increasing the representation of candidate populations in the genetic reference panel would significantly improve the resolution of the genetic assignment method for future analysis.

\section{Conclusions}

The presence of Ae. aegypti mosquitoes at Schiphol airport from flights arriving to the Netherlands, demonstrates the potential risk of international flights for public health. Identifying the origin of introduced invasive mosquito species is relevant to aid public health efforts to prevent the introduction and spread of these vectors of human-diseases. Collaborations between vector control agencies, airport authorities and genetic laboratories can facilitate tracking the origin of novel introductions. Furthermore, baggage compartments in aircrafts should be considered as potential pathways for the introduction of mosquitoes and, if disinsection is put in place, should also include these compartments in the aircraft.

\section{Acknowledgments}

We would like to thank Dr. Jeff R. Powell and Mr. Anton van der Sommen for their valuable suggestions and for critically reviewing the first drafts of the manuscript. 


\section{Additional file}

Additional file 1: Table S1. Genotypes of the five Aedes aegypti specimens captured at Schiphol, the Netherlands (2016) at 12 microsatellite loci. Loci AC1, AC2, AC4, AC5, CT2, AG1, AG2, and AG5 are dinucleotide repeats; loci A1, A9, B2, B3 are trinucleotide repeats as described in Gloria-Soria et al. 2016.

$\begin{array}{lrrrrrr}\text { Individual ID } & \text { AC1 } & \text { AC1 } & \text { AC2 } & \text { AC2 } & \text { AC4 } & \text { AC4 } \\ 802 \_2 & 197 & 197 & 184 & 188 & 128 & 130 \\ 845 \_2 & 195 & 209 & 186 & 188 & 130 & 130 \\ 853 & 209 & 209 & 184 & 188 & 128 & 128 \\ 6637071 & 209 & 197 & 186 & 184 & 128 & 128 \\ 6813416 & 195 & 197 & 186 & 188 & 128 & 130 \\ & & & & & & \\ \text { Individual ID } & \text { AC5 } & \text { AC5 } & \text { CT2 } & \text { CT2 } & \text { AG1 } & \text { AG1 } \\ 802 \_2 & 156 & 156 & 188 & 188 & 119 & 121 \\ 845 \_2 & 157 & 158 & 188 & 188 & 117 & 121 \\ 853 & 158 & 158 & 188 & 188 & 119 & 121 \\ 6637071 & 159 & 156 & 188 & 184 & 121 & 115 \\ 6813416 & 154 & 155 & 184 & 188 & 115 & 117 \\ & & & & & & \\ & & & & & & \\ \text { Individual ID } & \text { AG2 } & \text { AG2 } & \text { AG5 } & \text { AG5 } & \text { A1 } & \text { A1 } \\ 802 \_2 & 115 & 151 & 170 & 178 & 162 & 168 \\ 845 \_2 & 139 & 151 & 170 & 170 & 158 & 168 \\ 853 & 115 & 119 & 174 & 176 & 162 & 168 \\ 6637071 & 153 & 117 & 180 & 178 & 168 & 168 \\ 6813416 & 117 & 117 & 168 & 178 & 168 & 168 \\ & & & & & & \\ \text { Individual ID } & \text { A9 } & \text { A9 } & \text { B2 } & \text { B2 } & \text { B3 } & \text { B3 } \\ 802 \_2 & 188 & 188 & 113 & 113 & 166 & 166 \\ 845 \_2 & 182 & 191 & 95 & 95 & 172 & 172 \\ 853 & 188 & 191 & 113 & 113 & 175 & 175 \\ 6637071 & 191 & 191 & 113 & 113 & 166 & 166 \\ 6813416 & 191 & 191 & 107 & 113 & 166 & 166\end{array}$





\section{Chapter 8}

The effectiveness of Asian bush mosquito (Aedes japonicus japonicus) control actions in colonised peri-urban areas in the Netherlands

Adolfo Ibáñez-Justicia, Steffanie Teekema, Wietse den Hartog, Frans Jacobs, Marian Dik \& Arjan Stroo

Centre for Monitoring of Vectors (CMV), Netherlands Food and Consumer Product Safety Authority (NVWA), Wageningen, The Netherlands

Published in Journal of Medical Entomology (2018) 55(3):673-680, doi: 10.1093/jme/tjy002. 


\begin{abstract}
The Asian bush mosquito (Aedes japonicus japonicus (Theobald)) is an invasive mosquito species in Europe. In 2012, it was for the first time detected in the Netherlands, in the municipality of Lelystad. After further research, thousands of specimens were found in the surrounding peri-urban areas of the city. A targeted mosquito control campaign began in 2015 with the objective of reducing populations in locations with the highest concentrations of Ae. japonicus breeding sites: allotment garden complexes. Mosquito control consisted of source reduction combined with application of the larvicide Vectomax ${ }^{\mathrm{TM}}$ in breeding sites. At eight complexes, mosquito control effectiveness has been systematically measured by sampling larvae from breeding sites. Six measurements were performed between 2015 and 2016. Results show that the effectiveness of mosquito control actions was similar in all treated allotment gardens, and resulted in a significant reduction in Ae. japonicus larval abundance. Rain barrels at the allotments represent the most frequent breeding site in Lelystad, but every water filled artificial container is a potential breeding site for the species. Aedes japonicus was not found in the samples taken in other allotment gardens in the province of Flevoland; however, the collection methodology used proven to be effective in detecting this species when it has newly colonised surrounding areas. Targeted mosquito control actions at the breeding sites are crucial for successful reduction of populations of an invasive mosquito species, and systematic measurements of the effectiveness, is in this case, the base to understand the dynamics of Ae. japonicus populations after mosquito control.
\end{abstract}

Keywords: Mosquito, Aedes japonicus, vector, control, VectoMax. 


\section{Introduction}

The Asian bush mosquito Aedes japonicus japonicus (Theobald, 1901) is an invasive mosquito species (IMS) that originates from Japan, Korea and Southern China (Tanaka et al. 1979) and has also been found in south-eastern Russia (Gutsevich and Dubitskyi 1987). The first interception of the species in Europe occurred in France in year 2000 (Schaffner et al. 2003). Afterwards, established populations of this species have been found in Belgium (Versteirt et al. 2009), Switzerland (Schaffner et al. 2009), Germany (Becker et al. 2011, Kampen et al. 2012) (Werner and Kampen 2013), Austria and Slovenia (Seidel et al. 2012). Since the first interceptions in the 1990s Ae. japonicus has also successfully colonized numerous states of the USA, and by 2000 it was already reported from south-eastern Canada (Kampen and Werner 2014).

Under laboratory conditions, this species has been shown to be a competent vector of Japanese encephalitis virus (Takashima and Rosen 1989), West Nile virus (Sardelis and Turell 2001), La Crosse virus (Sardelis et al. 2002a), Eastern equine encephalitis virus (Sardelis et al. 2002b), St. Louis encephalitis virus (Sardelis et al. 2003), chikungunya virus and dengue virus (Schaffner et al. 2011). It has never been proven that Ae. japonicus is an efficient vector of pathogens in the field, but recent investigations in the United States have found La Crosse virus in field infected Ae. japonicus (Harris et al. 2015, Westby et al. 2015).

Extensive mosquito surveillance in 2013 confirmed the existence of a widely established population of Ae. japonicus in the municipality of Lelystad (Province of Flevoland, the Netherlands) (Ibañez-Justicia et al. 2014). This population seemed to be thriving in allotment gardens and in the surrounding patches of forest. Within the allotment gardens, Ae. japonicus larvae and pupae were found in multiple types of artificial containers. In 2014, a specific mosquito monitoring program in the province of Flevoland was implemented with the purpose of detecting populations of this invasive mosquito species in municipalities adjacent to Lelystad. Results showed that populations of Ae. japonicus in the province, seem to be confined to the municipality of Lelystad.

With the aim of preventing further spread of the population in the Netherlands, in 2015, the Ministry of Public Health decided to implement mosquito control actions in Lelystad (source reduction and control of larvae using larvicides) to reduce the population hotspots in allotment gardens. In combination with mosquito surveillance at the province of Flevoland to detect spread of the species, this control strategy was considered to be adequate for 
maintaining the populations of Ae. japonicus at low levels in order to prevent further spread. The ultimate goal of this control action is to achieve a medium/long term reduction of the populations in order to reduce the possible risk of vector-borne diseases outbreaks.

To achieve the goal of reducing the population of Ae. japonicus in Lelystad, Integrated Mosquito Management (IMM) is used. An IMM uses a combination of methods such as: mosquito surveillance, source reduction, control of larvae and pupae, and control of adults, to prevent and control mosquito vectors of diseases. Application of a targeted IMM at the locality of Natoye in Belgium (source reduction and larviciding) eliminated the existing population of Ae. japonicus between 2013 and 2015 (Direction Générale Opérationnelle Agriculture Ressources naturelles et Environnement 2017).

The aim of the study is 1) to evaluate the effectiveness of the mosquito control actions conducted in 2015 and 2016 in reducing the populations of Ae. japonicus at the mosquito hotspots in Lelystad, 2) to identify the most preferred breeding site in the allotment gardens, 3 ) to evaluate the reduction of the mosquito populations the Lelystad allotments compared to the populations in allotments from adjacent municipalities in the province, and 4) to study the population dynamics of Ae. japonicus through longitudinal mosquito surveillance at the infested locations.

\section{Material and methods}

\section{Study area}

The study area for evaluating the effectiveness of the control actions against Ae. japonicus consisted of allotment garden complexes $(n=8)$ totalling 22.68 hectares (Figure 1) in Lelystad (Table 1). Allotments in other municipalities within the province of Flevoland $(n=31)$, were also included in the study with the main objective of promptly detecting new populations of this IMS.

\section{Sampling methodology in Lelystad}

At eight allotments, mosquito control effectiveness was systematically measured. Using ArcMap 10.2.2 (ESRI, Redlands, USA), a grid of squares of 20x20 meters (400 square meters) was created for each allotment. Each square of the grid received a unique number. For each allotment, $30 \%$ of the total of squares were randomly chosen for study. At each random square, all potential breeding sites (containers) were sampled for presence and 
abundance of mosquito larvae. Six measurements were performed between 2015 and 2016, and at every measurement, sample squares were randomly selected from the grid. Measurements were performed between the months of March and November. The first larval measurement before the control actions started, the so called "zero measurement", was done in September 2015.

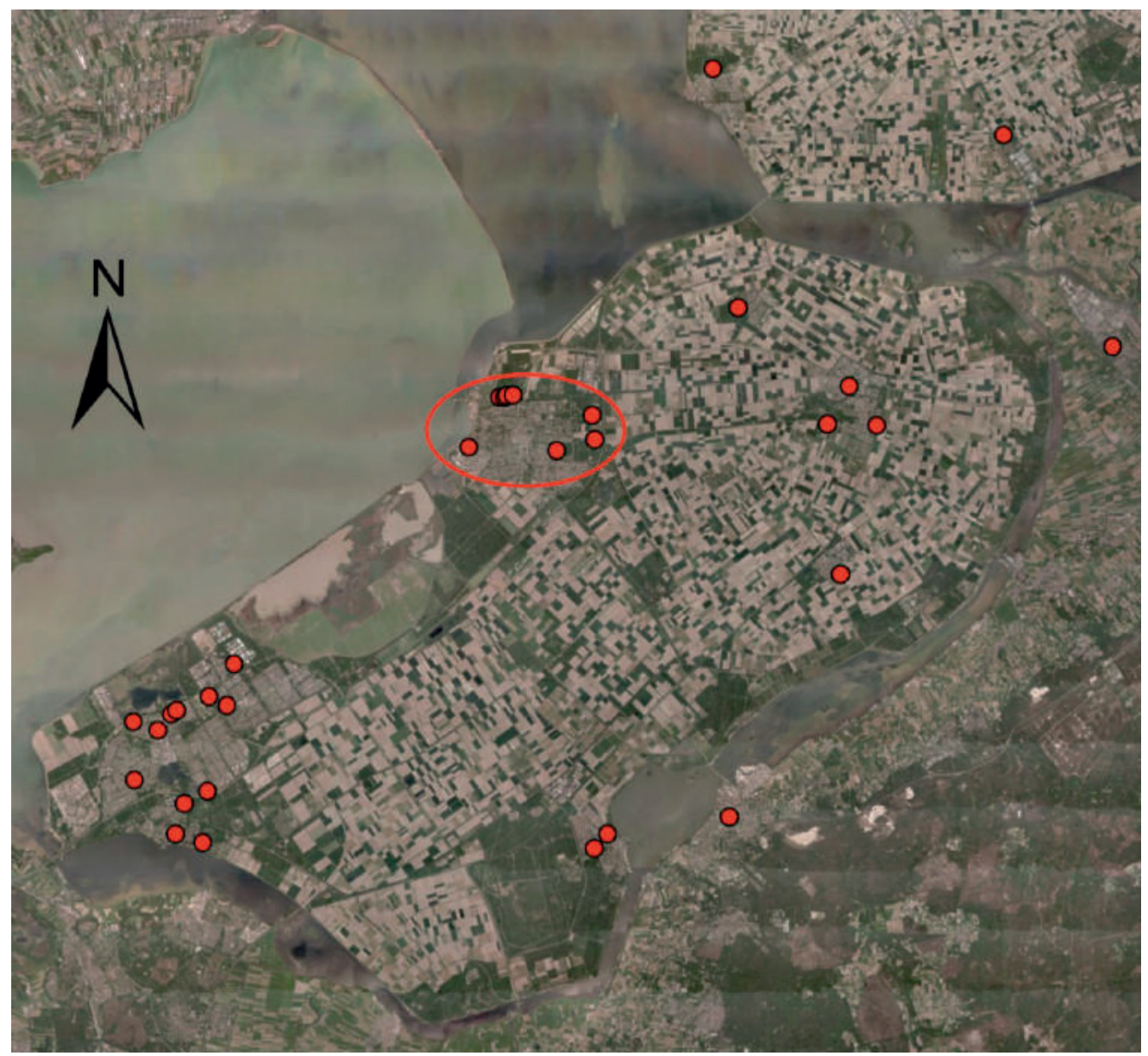

Figure 1. Location of the 31 monitored allotments in the Province of Flevoland and surroundings 20152016 (red points: allotments, red circle: Lelystad municipality).

At the chosen squares, the field inspector sampled all potential breeding sites (containers). At every container, and using aquarium fish nets (with fine mesh), the inspector collected all living invertebrates present. These were transferred to a white plastic container, and using a plastic pipette, all mosquito immatures (larvae and pupae) were collected and transferred to a plastic vial containing 70\% alcohol. Each vial was labelled with a barcoded sample number 
and samples were transferred to the laboratory. Each sample was accompanied by a paper form containing basic information about the sample including: date, location, square number, container type (rain barrel, bucket, litter, etc.), XY coordinates, and inspector name. Information about the total number and types of container in the measured square was also recorded.

Table 1. Study areas (allotments) measured to analyse the effectiveness of control measures in the municipality of Lelystad.

\begin{tabular}{lcccccc}
\hline Allotment name & $\begin{array}{c}\text { Area } \\
\text { (hectare) }\end{array}$ & $\begin{array}{c}\text { Total } \\
\text { number } \\
\mathbf{2 0 x 2 0 m} \text { grid } \\
\text { squares }\end{array}$ & $\begin{array}{c}\text { Area } \\
\mathbf{2 0 x 2 0 m} \\
\text { squares } \\
\text { (hectare) }\end{array}$ & $\begin{array}{c}\text { Number } \\
\text { squares } \\
\text { measured }\end{array}$ & $\begin{array}{c}\text { \% of } \\
\text { total } \\
\text { squares }\end{array}$ & $\begin{array}{c}\text { Area } \\
\text { measured } \\
\text { (hectare) }\end{array}$ \\
\hline Bosweg & 0.9 & 21 & 0.8 & 6 & $29 \%$ & 0.2 \\
Gelderse hout & 4.4 & 113 & 4.5 & 34 & $30 \%$ & 14 \\
Karveel & 1.9 & 46 & 1.8 & 14 & $30 \%$ & 0.6 \\
Ons Genoegen & 3.8 & 93 & 3.7 & 28 & $30 \%$ & 1,1 \\
Runderweg & 3.6 & 90 & 3.6 & 27 & $30 \%$ & 1.1 \\
Milieuvriendelijk tuinieren & 3.0 & 77 & 3.1 & 23 & $30 \%$ & 0.9 \\
Visarend & 2.6 & 64 & 2.6 & 19 & $30 \%$ & 0.8 \\
Zuigerplas & 2.4 & 60 & 2.4 & 18 & $30 \%$ & 0.7 \\
\hline Total & $\mathbf{2 2 . 7}$ & $\mathbf{5 6 4}$ & $\mathbf{2 2 . 6}$ & $\mathbf{1 6 9}$ & & $\mathbf{6 . 8}$ \\
\hline
\end{tabular}

\section{Sampling methodology in other allotment complexes in the Flevoland province}

To be able to detect Ae. japonicus in other allotments in the province of Flevoland, another monitoring programme was set up in 31 allotment complexes. (Figure 1). In these allotments, visual inspection of breeding sites was carried out. Among all mosquito breeding sites present at each allotment, rain barrels situated along the border of the allotment in proximity to a forested area were preferred for sampling. At each allotment, five open blue rain barrels containing water were selected and sampled for mosquito larvae. Sampling for mosquito immature stages was carried out following the same methodology mentioned before.

\section{Longitudinal adult sampling}

In 2016, longitudinal adult sampling of Ae. japonicus was carried out between April and November at two allotments. Two $\mathrm{CO}_{2}$-baited Mosquito Magnet Liberty Plus traps (Woodstream ${ }^{\circledR}$, Lititz, USA; hereafter MM trap) were checked weekly at each allotment. Traps were situated in similar conditions at each allotment, but separated at least $100 \mathrm{~m}$ to avoid trap interference. Trapping nets from the MM trap with collected mosquitoes were sent 
to the laboratory for morphological identification and were stored at $-20{ }^{\circ} \mathrm{C}$ until analysis. Field sampling information including: inspector name, site number, trap start date, trap end date, and XY coordinates recorded with a Global Positioning System (GPS) were documented and sent together with the sample.

\section{Mosquito diagnostics}

Field collected samples were sent to the National Reference Centre (NRC) laboratory of the Netherlands Food and Consumer Product Safety Authority (NVWA) for diagnostics. In the laboratory, mosquitoes were counted and morphologically identified by specialists using the electronic identification key of Schaffner (Schaffner et al. 2001), the key of Becker (Becker et al. 2010), and the key of Haren\&Verdonschot (Haren and Verdonschot 1995). Except for the target species (Aedes japonicus), larvae and pupae were identified to genus level (e.g. Culex sp., Culiseta sp., etc). Adults captured with the MM traps were identified to species level.

\section{Mosquito control}

In 2015 two actions were planned (in September and in October). In 2016, the first control action was conducted in April, and repetitions of the control actions were implemented every 4-5 weeks. Mosquito control consisted of elimination and treating of breeding sites with a larvicide (VectoMax ${ }^{\mathrm{TM}}$ granules FG, Libertyville, USA). If a breeding site could not be removed, granules were added to the water using a handheld dispenser. A standard dose of approximately 2 grams was applied to each container in the allotment. The dose was increased 2-3 fold for large containers (e.g. water tanks).

\section{Data analysis}

The general container index (GCI) and Ae. japonicus container index (JCI) were calculated at the eight Lelystad allotments for each of the six data collection time points as:

$\mathrm{GCI}=$ (number of breeding sites containing larvae/ total number of breeding sites) $\mathrm{x} 100$

$\mathrm{JCI}=$ (number of breeding sites containing Ae. japonicus larvae/ total number of breeding sites) $\mathrm{x} 100$

To test the mosquito control effects on the mosquito larval populations at the allotments, an analysis of the variance (ANOVA), was carried out on the GCI and JCI indices. Analysis was performed using software Genstat (version 17.1, 64 bit). To test for differences in mean number of larvae between Lelystad and the province of Flevoland, a Mann-Whitney U test 
was carried out with SPSS (SPSS Inc. Released 2008. SPSS Statistics for Windows, Version 17.0. Chicago: SPSS Inc).

\section{Results}

\section{Effectiveness mosquito control in Lelystad allotment gardens}

The total area of the eight allotments included in the mosquito control effort represented 22.68 hectares (Table 1). At every measurement, 6.8 hectares (30\% of the squares) were sampled for mosquito breeding sites. During the study in 2015-2016, mosquito larval presence was measured at six time points, two in 2015 (September and October), and four in 2016 (April, June, August, and November). Two rounds of mosquito control (source reduction and larviciding) were conducted in 2015, and seven in 2016. The period between mosquito control actions was planned to be a maximum of five weeks, corresponding to the maximum period of VectoMax ${ }^{\mathrm{TM}}$ larvicidal activity. Except for the period between the $5^{\text {th }}$ and $6^{\text {th }}$ control action in 2016 that lasted for 6 weeks, mosquito control actions were performed as planned.

Table 2 shows that the number of breeding sites found during the measurements in September $2015(n=808)$ and April $2016(n=818)$ are more numerous than the measurements in October $2015(n=592)$, June $(n=627)$, August $(n=585)$ and November $(n=630) 2016$. A total of 4,069 potential breeding sites were inspected, of which 3,396 sites were negative for the presence of mosquito immatures (83.46\%) (Table 2). Of the 673 breeding sites where mosquito larvae were detected $(16.53 \%)$, Ae. japonicus was recovered from 353 sites $(52.45 \%)$.

Results of the six measurements for the presence of mosquito larvae and specimens of $A e$. japonicus at each allotment are presented in Table 3. At the zero measurement in September 2015, GCI varied from $1.53 \%$ to $100 \%$ in the allotments. It can be noticed that Ae. japonicus was absent at the "Visarend" allotment at each measurement in the study. The maximum values of JCI at the zero measurement were recorded in the "Zuigerplas" $(61.73 \%)$ and "Bosweg" (60.87\%) allotments. Maximum values of GCI after the start of the mosquito control actions were recorded in August 2016 (32.43\% in "Zuigerplas" allotment and 32.35\% in "Bosweg" allotment). At the last measurement in November 2016, all allotment complexes 
were negative for the presence of Ae. japonicus mosquito immatures except in the "Runderweg" allotm ent (JCI 1.33\%).

Table 2 . Breeding site measurements and container indices at the allotments of Lelystad. na $=$ not applicable.

\begin{tabular}{lccccccc}
\hline \hline All allotments & Sep-15 & Oct-15 & Apr-16 & Jun-16 & Aug-16 & Nov-16 & Total \\
\hline No. potential breeding sites inspected & 808 & 592 & 818 & 627 & 585 & 639 & 4,069 \\
No. breeding sites larvae absent & 410 & 576 & 780 & 542 & 455 & 633 & 3,396 \\
No. breeding sites with larvae & 398 & 16 & 38 & 85 & 130 & 6 & 673 \\
No. breeding sites with Ae. japonicus & 242 & 10 & 36 & 7 & 57 & 1 & 353 \\
General container index \% (GCI) & 49.25 & 2.70 & 4.64 & 13.55 & 22.22 & 0.93 & na \\
Ae. japonicus container index \% (JCI) & 29.95 & 1.68 & 4.40 & 1.11 & 9.74 & 0.15 & na \\
\hline
\end{tabular}

In the 673 breeding sites containing larvae, a total of 44,461 mosquito larvae were identified. Larval identifications for each allotment are presented in Table 4. Culex sp. larvae were the most abundant in the containers in the allotments of Lelystad ( $\mathrm{n}=37,319,83.93 \%)$ followed by Ae. japonicus larvae $(\mathrm{n}=6,509,14.63 \%)$, and Culiseta sp. $(\mathrm{n}=586,1.31 \%)$. Anopheles sp. larvae and Aedes/Ochlerotatus larvae (other than Ae. japonicus) were also found in a lesser extent. Table 5 shows the total number of larvae mosquito specimens identified at each of the six measurements. During the zero measurement in September 2015, the highest number of larvae were identified ( $\mathrm{n}=18,738)$, including the highest number of Culex sp. $(\mathrm{n}=14,354)$, and Ae. japonicus $(\mathrm{n}=4,335)$. The last measurement in November 2016 provided the lowest results in collection of mosquito larvae $(n=225)$. At the measurement in April 2016, larvae of Culex sp. were absent in the samples and Ae. japonicus was present $(\mathrm{n}=862)$.

During the study period, Aedes japonicus larvae were found in a total of 353 breeding sites. Rain barrels at the allotments were the most frequent breeding site found for Ae. japonicus in Lelystad. As shown in figure 2, forty-seven percent $(n=166)$ of the containers where Ae. japonicus has been found were rain barrels. Other frequent breeding sites were buckets $(n=49)$ and small bins $(n=48)$ but it was also been found breeding in a variety of different containers. 
Table 3. Results of General Container Index (GCI \%) and Aedes japonicus Container Index (JCI \%) at the eight allotments included in mosquito control. Allotment names abbreviations: Bos= Bosweg; Ge= Gelderse Hout; Kar= Karveel; Ons= Ons genoegen; Mil= Milieuvriendelijk tuineren; Run= Runderweg; Vis= Visarend; Zui= Zuigerplas.

\begin{tabular}{lcccccccc}
\hline \hline & Bos & Bos & Gel & Gel & Kar & Kar & Ons & Ons \\
& GCI & JCI & GCI & JCI & GCI & JCI & GCI & JCI \\
\hline Sep-15 & 69.5 & 60.8 & 20.3 & 2.7 & 45.0 & 27.5 & 44.0 & 31.1 \\
Oct-15 & 4.5 & 3.0 & 0.0 & 0.0 & 2.2 & 0.0 & 0.0 & 0.0 \\
Apr-16 & 5.9 & 5.9 & 0.0 & 0.0 & 3.6 & 1.8 & 6.0 & 6.0 \\
Jun-16 & 12.9 & 3.2 & 17.3 & 0.0 & 21.4 & 0.0 & 17.2 & 2.1 \\
Aug-16 & 32.3 & 26.4 & 20.5 & 0.9 & 19.7 & 9.1 & 18.2 & 6.3 \\
Nov-16 & 2.0 & 0.0 & 0.0 & 0.0 & 1.8 & 0.0 & 0.0 & 0.0 \\
\hline
\end{tabular}

\begin{tabular}{lcccccccc}
\hline \hline & Mil & Mil & Run & Run & Vis & Vis & Zui & Zui \\
& GCI & JCI & GCI & JCI & GCI & JCI & GCI & JCI \\
\hline Sep-15 & 55.8 & 41.4 & 100.0 & 40.9 & 1.5 & 0.0 & 70.4 & 61.7 \\
Oct-15 & 9.5 & 6.7 & 2.7 & 1.4 & 0.0 & 0.0 & 0.0 & 0.0 \\
Apr-16 & 10.8 & 10.8 & 4.0 & 4.0 & 0.0 & 0.0 & 4.2 & 4.2 \\
Jun-16 & 11.5 & 0.0 & 12.4 & 2.5 & 0.0 & 0.0 & 10.5 & 0.0 \\
Aug-16 & 21.4 & 11.4 & 20.4 & 12.6 & 1.8 & 0.0 & 32.4 & 16.2 \\
Nov-16 & 0.0 & 0.0 & 1.3 & 1.3 & 0.0 & 0.0 & 6.1 & 0.0 \\
\hline
\end{tabular}

\section{Total breeding sites Ae. japonicus}

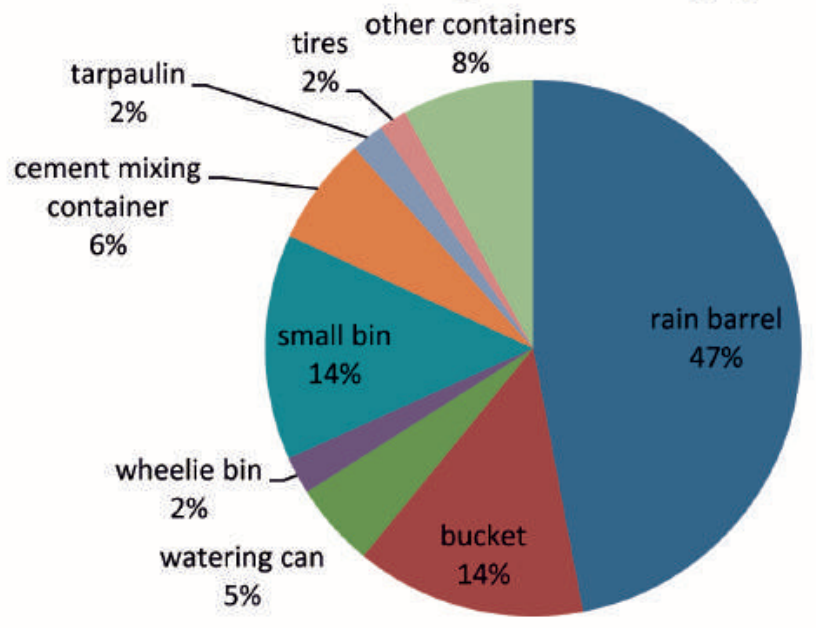

Figure 2. Chart showing proportion of breeding site types with Ae. japonicus larvae, at the monitored allotments in 2015 and 2016 in Lelystad. 
The ANOVA analysis to check the differences on GCI and on JCI at the eight allotments indicated that there were no significant differences in the calculated indices between the allotments (ANOVA GCI: $\mathrm{df}=6, \mathrm{~F}=1.14, \mathrm{P}=0.364$; ANOVA JCI: $\mathrm{df}=6, \mathrm{~F}=2.25, \mathrm{P}=$ 0.06). However, there is a significant difference $(\mathrm{P}<0.001)$ between the sampling dates. Mean percentage of GCI and JCI data, and least significant difference grouping is presented in Table 6. Statistically significant differences on GCI and on JCI were noted between the zero measurement and the rest of the sampling dates $(\mathrm{P}<0.001)$.

Table 4. Number of samples and number of larvae of Aedes japonicus and other mosquitoes at the eight allotments in Lelystad included in mosquito control in 2015 and 2016.

\begin{tabular}{|c|c|c|c|c|c|c|c|}
\hline $\begin{array}{l}\text { Allotment } \\
\text { complex name }\end{array}$ & $\begin{array}{c}\text { Number } \\
\text { of samples }\end{array}$ & $\begin{array}{l}\begin{array}{c}\text { Culex } \\
\text { sp. }\end{array} \\
\end{array}$ & $\begin{array}{c}\text { Aedes } \\
\text { japonicus }\end{array}$ & $\begin{array}{c}\text { Culiseta } \\
\text { sp. }\end{array}$ & $\begin{array}{c}\text { Other } \\
\text { Aedes sp. }\end{array}$ & $\begin{array}{c}\text { Anopheles } \\
\text { sp. }\end{array}$ & $\begin{array}{l}\text { Total } \\
\text { larvae }\end{array}$ \\
\hline Bosweg & 40 & 2,559 & 623 & 190 & 0 & 1 & 3,373 \\
\hline Gelderse Hout & 71 & 7,958 & 9 & 84 & 0 & 0 & 8,051 \\
\hline Karveel & 49 & 3,557 & 250 & 105 & 12 & 2 & 3,926 \\
\hline Ons Genoegen & 156 & 8,993 & 1,485 & 39 & 0 & 8 & 10,525 \\
\hline $\begin{array}{l}\text { Milieuvriendelijk } \\
\text { tuinieren }\end{array}$ & 106 & 3,395 & 1,918 & 157 & 0 & 14 & 5,484 \\
\hline Runderweg & 159 & 4,808 & 1362 & 8 & 4 & 4 & 6,186 \\
\hline Visarend & 2 & 28 & 0 & 0 & 0 & 0 & 28 \\
\hline Zuigerplas & 90 & 6,021 & 862 & 3 & 0 & 2 & 6,888 \\
\hline Total & 673 & 37,319 & 6,509 & 586 & 16 & 31 & 44,461 \\
\hline
\end{tabular}

Table 5. Number of Aedes japonicus larvae and larvae of other genera in the eight Lelystad allotments included in mosquito control in 2015 and 2016.

\begin{tabular}{lcccccc}
\hline $\begin{array}{l}\text { Measurement } \\
\text { moment }\end{array}$ & $\begin{array}{c}\text { Culex. } \\
\text { sp }\end{array}$ & $\begin{array}{c}\text { Aedes } \\
\text { japonicus }\end{array}$ & $\begin{array}{c}\text { Culiseta } \\
\text { sp. }\end{array}$ & $\begin{array}{c}\text { Other } \\
\text { Aedes sp. }\end{array}$ & $\begin{array}{c}\text { Anopheles } \\
\text { sp. }\end{array}$ & Total \\
\hline Sep-15 & 14,354 & 4,335 & 40 & 0 & 9 & 18,738 \\
Oct-15 & 220 & 233 & 1 & 0 & 2 & 456 \\
Apr-16 & 0 & 862 & 4 & 12 & 0 & 878 \\
Jun-16 & 11,436 & 92 & 50 & 0 & 2 & 11,580 \\
Aug-16 & 11,287 & 980 & 295 & 4 & 0 & 12,584 \\
Nov-16 & 22 & 7 & 196 & 0 & $\mathbf{3 1}$ & $\mathbf{4 4 , 4 6 1}$ \\
\hline Total & $\mathbf{3 7 , 3 1 9}$ & $\mathbf{6 , 5 0 9}$ & $\mathbf{5 8 6}$ & $\mathbf{1 6}$ & \\
\hline
\end{tabular}

\section{Comparison Lelystad with measured allotments in Flevoland}

In 2015 and 2016 in a maximum of 28 allotments in the province of Flevoland (including Lelystad), samples were taken that could be used for assessing the effect of the mosquito control in the municipality of Lelystad. A total number of 42,706 mosquito larvae were 
collected and identified in the samplings conducted in May and in September of the years 2015 and 2016. Measuring only five rain barrels in this survey in the allotments in Lelystad it was possible to detect Ae. japonicus in almost all samples. All samples taken in the allotments outside Lelystad were negative for the presence of Ae. japonicus. Results show a clear decrease in the average number of mosquito larvae in Lelystad allotment rain barrels from May 2015 onwards, ranging from a maximum of 205.19 larvae per rain barrel in May 2015 to a minimum of 6.99 in September 2016. In the allotments of Flevoland (outside Lelystad), the average number of larvae per rain barrel decreased from 165.42 in May 2015 to 20.79 in May 2016. However, in September 2016, unlike in Lelystad, the average number of larvae per rain barrel increased to 42.44 (Table 7). Only the data of Lelystad and the adjacent municipality of Almere were used in the analysis because the allotments in these municipalities were most extensively sampled. The data from other municipalities in the province of Flevoland appeared to be insufficient for the analysis. The Mann-Whitney U test revealed that the average number of larvae found in Lelystad (Mean Rank=4.75, $n=8$ ) was significantly lower than in Almere (Mean Rank=13.3, $\mathrm{n}=10$ )(Mann-Whitney $\mathrm{U}=2.00$, $\mathrm{p}=0.001$ and $\mathrm{p}<0.05$ ) only at the measurement in September 2016. All the other measurements (May and September 2015, and May 2016), showed no significant differences in the average number of larvae collected.

Table 6. Fisher's protected least significant difference test grouping results for G eneral Container Index (GCI) and Aedes japonicus Container Index (JCI) for the eight Lelystad allotments included in mosquito control in 2015 and 2016.

\begin{tabular}{llllll}
\hline \hline GCI & \multicolumn{5}{c}{ JCI } \\
\hline Moment & Mean & Group & Moment & Mean & Group \\
\hline November 2016 & 1.6 & $\mathrm{a}$ & November 2016 & 0.2 & $\mathrm{a}$ \\
October 2015 & 2.7 & $\mathrm{a}$ & October 2015 & 1.6 & $\mathrm{ab}$ \\
April 2016 & 4.9 & $\mathrm{ab}$ & June 2016 & 2.6 & $\mathrm{ab}$ \\
June 2016 & 15.0 & $\mathrm{bc}$ & April 2016 & 4.7 & $\mathrm{ab}$ \\
Augusts 2016 & 23.4 & $\mathrm{c}$ & Augusts 2016 & 10.4 & $\mathrm{~b}$ \\
September 2015 & 57.9 & $\mathrm{~d}$ & September 2015 & 38.0 & $\mathrm{c}$ \\
\hline
\end{tabular}

\section{Longitudinal data}

The first adults captured in the MM trap were recorded on 13th May 2016 (week 19). Since that collection, adults of Ae. japonicus were captured every week in the traps placed in Lelystad through 28 October 2016 (week 43). A total of 416 adults were captured in the four 
traps. The maximum number of Ae. japonicus (37) captured in a single trap was recorded between 14 July and 22 July, followed by a capture of 27 specimens between 15 th and 22 th of September (Figure 3).

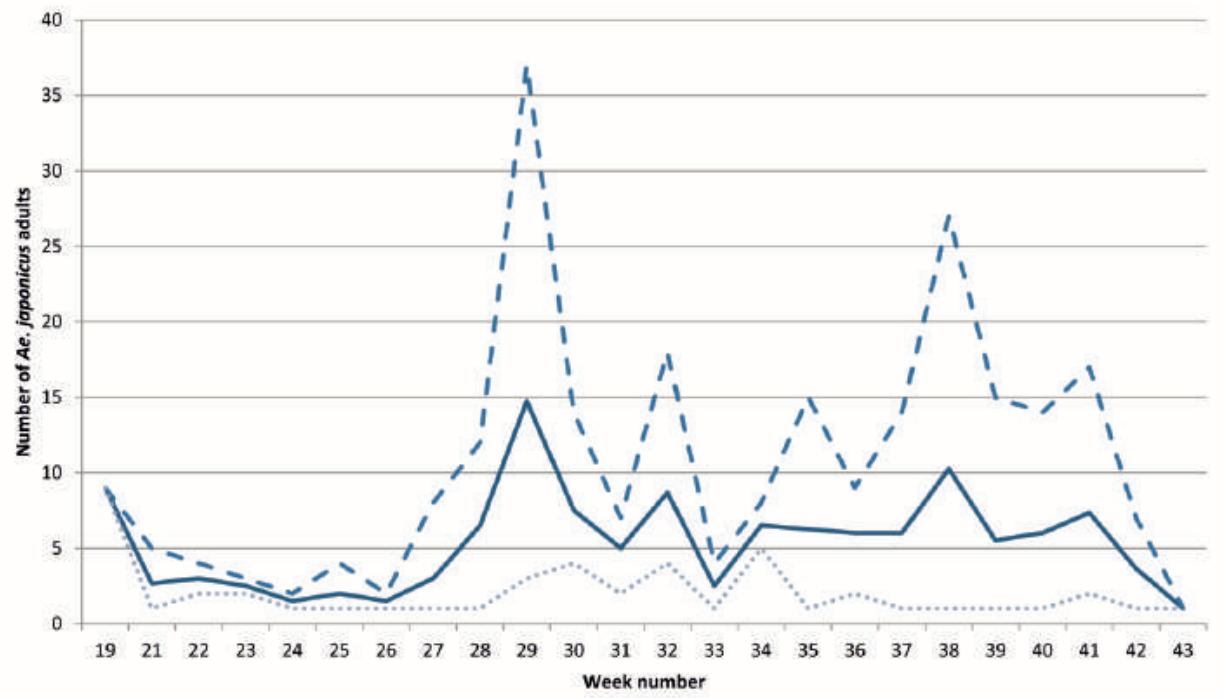

Figure 3. Minimum, maximum and average number of Aedes japonicus adults captured in longitudinal survey in Lelystad in 2016. Dotted line = minimum number; dashed line $=$ maximum number; solid line $=$ average number.

Table 7. Average number (and standard deviation) of mosquito larvae found in rain barrels in Lelystad and Flevoland surveillance for Aedes japonicus in 2015-2016.

\begin{tabular}{llclcc}
\hline Year & Location & $\begin{array}{c}\text { Number } \\
\text { allotments }\end{array}$ & Moment & $\begin{array}{c}\text { Average number of mosquito } \\
\text { larvae in rain barrel }\end{array}$ & $\begin{array}{c}\text { SD Standard } \\
\text { deviation }\end{array}$ \\
\hline 2015 & Flevoland & 21 & May & 165.4 & 165.6 \\
2015 & Flevoland & 19 & September & 91.1 & 74.3 \\
2016 & Flevoland & 20 & May & 20.8 & 27.9 \\
2016 & Flevoland & 20 & September & 42.4 & 41.8 \\
2015 & Lelystad & 7 & May & 205.2 & 150.0 \\
2015 & Lelystad & 7 & September & 48.4 & 30.3 \\
2016 & Lelystad & 8 & May & 24.4 & 29.1 \\
2016 & Lelystad & 8 & September & 7.0 & 10.9 \\
\hline
\end{tabular}

\section{Discussion}

In this article, we report the measured effectiveness of the largest active mosquito control action taken in the Netherlands since the vector control measures against malaria vectors (van 
Seventer 1969). To date, actions taken against Ae. japonicus have only been effective in Belgium, eliminating this mosquito vector species around the town of Natoye (Direction Générale Opérationnelle Agriculture Ressources naturelles et Environnement 2017). In an area of more than 22 hectares in Lelystad, source reduction combined with the application of larvicide was performed, and in more than 6 hectares, the effectiveness of these treatments was measured. For this task, a GIS based methodology was applied. We consider that the calculated larval indices at the allotment complexes during the different measurements have been proven to be effective for statistical comparison. In other parts of Europe, the number of mosquito pupae per hectare (PHI) has been applied as an index and could provide useful data (Petric et al. 2014). Pupal indices exploit the strong correlation between the number of pupae and the number of adults in a defined area, based on the low natural mortality of the pupae (Baldacchino et al. 2015). Due to the large amount of specimens collected in our study, it was decided to focus the efforts only on the larval indices.

An especially relevant measurement, was the measurement zero in September 2015, which showed the extent of the population. In some allotments Ae. japonicus larvae were present in more than $60 \%$ of the containers sampled. It can be noticed that at the "Visarend" allotm ent, that larval indices were always very low, and Ae. japonicus was absent at each measurement in the study. Larvae of Ae. japonicus were found in the allotment during the intensive surveillance of the municipality in 2013 (Ibañez-Justicia et al. 2014). The "Visarend" allotment is situated in the vicinity of a large used tire company that, since 2013, has been found positive for introductions of Ae. albopictus every year. Control measures applied in this location after finding an invasive mosquito include, among others, larviciding an area of 500 meters surrounding the site. As a result, this allotment has been treated since 2013 with larvicide, resulting in a reduction in mosquito populations breeding in the containers and in the absence of Ae.japonicus during this study.

Finding lower numbers of breeding sites present during the measurements after the first mosquito control actions in 2015 (September) and in 2016 (April), confirm the expected results of the source reduction at the allotments. However, following the measurement in June 2016, the number of potential breeding sites did not decrease any further. The use of rain barrels is the most common practice in the allotments to collect and store water. At least one or two rain barrels are encountered at each parcel and even in a mosquito control campaign to reduce populations of an invasive mosquito species, these potential breeding 
sites could not be easily removed. Since the number of rain barrels could not be further reduced, those remaining were treated with larvicide.

In comparison with other potential breeding sites, Ae. japonicus larvae were most frequently found in rain barrels. Other breeding sites where Ae. japonicus frequently occurred were buckets and small bins. In addition to these breeding sites, Ae. japonicus was also found in a variety of different containers such as watering cans, wheelie bins, cement mixing containers, tarpaulins, and used tires. In the measurements at the allotments, all water filled artificial containers were considered to be potential breeding sites for Ae.japonicus.

Culex sp. larvae were the most abundant in the containers at the allotments of Lelystad followed by Ae. japonicus larvae. It needs to be noticed that at the measurement in April 2016, larvae of Culex sp. were absent in all samples taken, and almost all larvae found were Ae. japonicus. These Ae. japonicus larvae were probably larvae from late 2015 that passed the winter in the containers. This information is important because, mosquito control (source reduction or larviciding) applied at this moment will only affect larvae of the target invasive mosquito species.

The effect of the mosquito control actions (source reduction and larviciding) on population reduction at the eight allotments in Lelystad has been proven for all larvae species (GCI) and for Ae. japonicus (JCI). Both larval indices were high at the zero measurement, and low in the remaining sampling periods. The effects of the treatments was proven to be similar at the eight locations. Lowest values of larval indices were found, and lowest amount of larvae were collected after the last treatment (November 2016). As published by (Schaffner et al. 2013), limiting the available sites for oviposition through source reduction also affects the distribution of native mosquitoes such as Culex sp. in a locality. According to the statistical analysis (Fisher's protected LSD test), the low value of JCI found at the last measurement in November $2016(0.190 \%)$ was not significantly different from the value of JCI in October 2015, June 2016 or April 2016. At these moments, values of JCI can be also considered low. The value of JCI found in August 2016 (10.441\%) is significantly higher than the value of JCI in November 2016 (0.190\%), but it not significantly different from the values of June and April 2016, and October 2015.

The methodology applied for sampling larvae at the allotments in Flevoland province proved to be appropriate to detect the presence of Ae. japonicus. Using this sampling methodology (larval sampling in five rain barrels containing larvae preferably nearby forested areas ) Ae. 
japonicus was detected in Lelystad even after the control actions when populations were lower. We consider that if Ae. japonicus was present at the other allotments in the province, it would have been detected during the study. Results from collections in Lelystad using this sampling methodology also show a clear decrease in the average number of mosquito larvae in the rain barrels in the allotments from May 2015 onwards. This decreasing larval density trend was not seen in the sampled allotments in province of Flevoland, where the average of larvae per rain barrel decreased from May 2015 to May 2016, but increased in September 2016. This indicates that the control actions taken in Lelystad do have an effect on the m osquito population at the 'hot spots'.

Longitudinal data of adult Ae. japonicus captured with MM traps revealed important biological facts for this species in the Netherlands. Traps were deployed at the end of April, but first captures were recorded after the second weekly control. We can consider that the first adults collected in these allotments originated from overwintering larvae that developed to adults when an appropriate temperature was reached to complete their life cycles. From that collection forward, adult specimens were captured in the traps every week with low numbers from May until early July and reaching a peak in the middle of July. Adults emerging in July could have originated from eggs laid by the adults that emerged in May, or remaining eggs from 2015 that hatched in 2016 when ecological conditions were appropriate for survival of the larvae (contact with water, appropriate temperature, and appropriate photoperiod). Two months later, in September, another peak in the population was recorded. In this case, the majority of the adults probably originated from eggs laid by adult females emerging in 2016.

For the first time, results of effectiveness of the mosquito control actions performed to reduce populations of Aedes japonicus are reported in Europe. Populations of the target species were considerably reduced after the applied mosquito control actions. As recommended by (Baldacchino et al. 2015), source reduction methods, should involve the public in a community-based approach. Effective source reduction, requires scrupulous and repeated cleaning or treatment of containers for everyday use, and so relies on extensive homeowner collaboration (Unlu et al. 2013). A community-based participation program, could significantly reduce the cost of control measures and contribute greatly to the decrease of the IMS populations in Lelystad in the future. However, it is not expected that source reduction in combination with larvicide intervention will completely eliminate the populations at the allotments, due the presence of untreated cryptic containers in the surrounding areas. 
Effective and targeted mosquito control actions at the breeding sites are crucial for successful reduction of populations of an IMS, and well-planned and systematic measurement of the effectiveness, is in this case, the base to understand the dynamics on the populations of $A e$. japonicus after mosquito control. For this reason, monitoring in allotments of the Flevoland province is recommended for promptly detecting the possible spread of the species from Lelystad, and if necessary, applying control measures.

\section{Acknowledgments}

We would like to thank Dr. Scott Gordon for reviewing the English in the manuscript, the colleagues of the Netherlands Food and Consumer Product Safety Authority (NVWA) for assistence in the field and laboratory work, and the allotment owners for giving us permission for sampling on their properties. 



\section{Chapter 9}

\section{Habitat suitability modelling to assess the introductions of Aedes albopictus (Diptera, Culicidae) in the Netherlands}

Adolfo Ibáñez-Justicia ${ }^{1}$, Ron van Lammeren ${ }^{2}$, Constantianus J.M. Koenraadt ${ }^{3}$, Aldo Bergsma $^{2}$, Luca Delucchi ${ }^{4}$, Annapaola Rizzoli ${ }^{4} \&$ Willem Takken ${ }^{3}$

1. Centre for Monitoring of Vectors (CMV), Netherlands Food and Consumer Product Safety Authority (NVWA), Wageningen, The Netherlands

2. Laboratory of Geo-information Science and Remote Sensing, Wageningen University \& Research, Wageningen, The Netherlands

3. Laboratory of Entomology, Wageningen University \& Research, Wageningen, The Netherlands

4. Research and Innovation Centre, Fondazione Edmund Mach, San Michele all'Adige, Italy 


\begin{abstract}
In the Netherlands, Aedes albopictus has been found each year since 2010 during routine exotic mosquito species surveillance at companies that import used tires. To investigate the potential risk of establishment and spread of this invasive species at these locations, habitat suitability models have been developed. Two methodologies have been used: first, a species distribution model based on the maximum entropy niche modelling approach (MAXENT) taking into consideration the most updated occurrence data of the species in Europe, and second, a spatial logic conditional model based on the temperature requirements of the species and using Land Surface Temperature data (LST-model). Results show differences in predicted habitat suitability values and patterns. The results of the MAXENT model are more restrictive for the species, the winter temperatures being the variable that contributed most to the performance of the model. The results of the LST-model show that 1) coastal areas are suitable for overwintering of eggs, 2) large areas in the northern part of the country have a low suitability for adult survival, and 3) the entire country is suitable for successful completion of the life cycle if the species is introduced after the winter months. Results of the LST-model reveal that temperatures in 2012 and 2014 did not limit the overwintering of eggs or survival of adults at the used tire companies where the species was found. By contrast, in 2010, 2011 and 2013, overwintering of eggs at these locations is not considered likely. The results obtained in our study aid on the design of efficient monitoring activities and risk assessments, and lead to more effective methods to control and prevent further spread of the species, if introduced in The Netherlands.
\end{abstract}

Keywords: invasive mosquitoes, habitat suitability models, land surface temperature, MAXENT, GIS 


\section{Introduction}

Aedes albopictus (Skuse, 1895) (Diptera: Culicidae) has its origin in South-East Asia, but due to global trade and transport the species has expanded its distribution range to all continents of the world except Antarctica (Benedict et al. 2007). Aedes albopictus was introduced into the United States in 1985, most likely via import of used tires from Japan (Sprenger and Wuithiranyagool 1986). The first sighting in Europe came from Albania in 1979, and probably originated from goods imported from China (Adhami and Reiter 1998). However, it was only when the species was introduced into Italy in 1990 through the import of used airplane tires from Atlanta (USA) (Dalla Pozza and Majori 1992), that the species became established throughout Italy. From the initial introduction location in Italy, the species gradually spread to many European countries (ECDC 2016). In its native range, Ae. albopictus was considered as a rural mosquito (Higa 2011), breeding preferably in natural habitats and being mainly present at the edge of forests. Outside its native range, the species has successfully adapted to local conditions and, in many of these areas, became established. The species is characterized by its ecological plasticity and has adapted to breed in various types of man-made waterfilled containers (tires, bins, rain barrels, etc.) in suburban and urban areas. Aedes albopictus is an undesirable invasive mosquito species (IMS), that causes considerable nuisance by its biting behaviour (Kamgang et al. 2012), and the species has been proven to transmit more than 22 different viruses under laboratory conditions (Gratz 2004). In the field, it is considered a competent vector of chikungunya and dengue viruses (Delatte et al. 2008). Furthermore, several other pathogens were isolated from specimens collected in the field such as West Nile virus, Eastern equine encephalitis virus (Mitchell et al. 1992) and LaCrosse virus (Gerhardt et al. 2001) in North America, and the heartworms Dirofilaria immitis and D. repens and Usutu virus in Italy (Cancrini et al. 2007, Puggioli et al. 2017). Since its emergence and establishment in southern Europe, Ae. albopictus has been associated with autochthonous cases of several diseases considered "tropical" in Europe, such as chikungunya in Italy (Rezza et al. 2007, Venturi et al. 2017), dengue and chikungunya fever in France (Gould et al. 2010, La Ruche et al. 2010, Calba et al. 2017) and dengue in Croatia (Gjenero-Margan et al. 2010).

As an ectothermic arthropod, the physiology of Ae. albopictus is determined by environmental variables, mainly temperature, and these will affect its geographical distribution. Data of the current geographical distribution and the ecological and physiological requirements of the species, in combination with geographic data of specific environmental variables, can be used to create habitat suitability maps to investigate the potential risk of 
establishment and spread of this invasive species. Considering its public health threat and invasiveness, several studies have already been conducted at continental scale using habitat suitability models driven by environmental variables (Benedict et al. 2007, ECDC 2009, Fischer et al. 2011, Caminade et al. 2012, Proestos et al. 2015, Kraemer et al. 2015a, Cunze et al. 2016a). Interestingly, the results of these studies at European scale show different prediction outcomes, especially for the northern distribution limits of the species (e.g. United Kingdom, the Netherlands, northern Germany). Given these differences between the published predictions so far, the probability of becoming established following accidental introduction remains unclear for the Netherlands. For example, the studies published for Ae. albopictus of Benedict et al. (2007), ECDC (2009), Kraemer et al. (2015a) show a low suitability for the species in the Netherlands compared to southern Europe and the Mediterranean basin. Other studies based on ecological niche models show a moderate suitability in the southern parts of the country (Fischer et al. 2011, Cunze et al. 2016a). In the habitat suitability study of Proestos et al. (2015) the predicted suitability is high for the Netherlands.

Habitat suitability models have also been applied at national and regional scale in Italy, Switzerland and Germany (Neteler et al. 2011, Roiz et al. 2011, Neteler et al. 2013, Koch et al. 2016). A study specifically focused on the Netherlands (Takumi et al. 2009), using the environmental variables temperature, precipitation and photoperiod, assessed the possibility for establishment following the accidental introductions in 2005 and 2006 at Lucky bamboo greenhouses (Scholte et al. 2007). Results of this study suggested that the winter conditions in the Netherlands could be permissive for the establishment of temperate strains of Ae albopictus. Since 2010, Ae. albopictus has been found each year during routine exotic mosquito species surveillance at companies that import used tires (See Chapter 6 in this thesis), and in several locations, the findings occurred at companies that were found positive the year before, suggesting that at these locations, new sets of used tires containing eggs of Ae. albopictus were introduced and stored outside, leading to new findings the following year. We would like to investigate whether the species could successfully overwinter at these locations, or whether the species was reintroduced each year again through import of infested tires. As mosquito control is routinely applied in The Netherlands after the findings, this information is important, because it is essential to know at which locations and in which year diapausing eggs could survive during the winter, and how suitable are these locations for the survival of adults if mosquito eggs are introduced after the winter months. 
To answer this question we created habitat suitability maps for Ae. albopictus in the Netherlands by using two methodologies. In the first methodology we constructed a suitability map using a species distribution model based on the maximum entropy niche modelling approach (we will refer to this model as the MAXENT model), taking into consideration the most updated occurrence data of the species in Europe (only considering confirmed overwintering of the species at the occurrence location). In the second, we created a habitat suitability map based on a spatial logic conditional model using the temperature requirements of the species measured in other studies, as well as Land Surface Temperature (LST) data (we will refer to this model as the LST-model). These suitability maps are then contrasted with the findings of Ae. albopictus during surveillance in used tire companies in the Netherlands, to establish relationships considering the possibility of overwintering of the species at these locations, or relating the findings to new introductions (e.g. with import of used tires). Exploring the habitat suitability of the Netherlands for sustaining populations of Ae. albopictus will help to understand the risk for vector-borne diseases associated with the establishment of populations of this species in the country. This may also aid the design of efficient monitoring activities and risk assessments, and lead to more effective methods to control and prevent further spread of the species, if introduced in The Netherlands.

\section{Materials and Methods}

\section{Study Area}

The spatial extent of the case study area contains the complete territory of the Netherlands: latitude $50.75 \mathrm{~N}-53.55 \mathrm{~N}$, longitude $3.35 \mathrm{~W}-7.22 \mathrm{E}$. The study area is characterized by a flat landscape with large areas occupied by lakes, rivers and canals. The overall study area accounts $34,933 \mathrm{~km}^{2}$ not including the inland waters. About $25 \%$ of the land is at, or below sea level. The climate is temperate, with mild winters, cool summers, and rainfall in every season. Urban and peri-urban areas represent approximately $20 \%$ of the country total area (Ibañez-Justicia et al. 2015). For the MAXENT model we originally used a larger extent (Europe): Latitude, $28.98 \mathrm{~N}-73.22 \mathrm{~N}$; Longitude, 16.30W - 48.25E. However, for comparison with the results of the LST-model we extracted the results of MAXENT model accordingly to the spatial extent of the Netherlands. 


\section{Environmental data}

For the LST-model, we used the data acquired from the MODIS sensor. The MODIS sensor (Moderate Resolution Imaging Spectroradiometer) is specifically designed to capture environmental variables on board of the satellites Terra and Aqua (https://modis.gsfc.nasa.gov/data/). The LST from MODIS is acquired with four records per day with a spatial resolution of $1,000 \mathrm{~m}$. In our LST-model we used the daily averaged LST data from 1/1/2009 until 31/12/2015 (see Table 1). The original pixel resolution of 1,000 m was increased to $250 \mathrm{~m}$. This data was provided and processed for the study area by the Edmund Mach Foundation (San Michele all'Adige, Italy). For further details of the processing of the daily averaged LST data see Metz et al. (2014).

Table 1. Data frame environmental variables used in the LST and MAXENT model.

\begin{tabular}{lll}
\hline \hline & LST-model & MAXENT model \\
\hline Source & MODIS sensor & WorldClim \\
Environmental variable(s) & Land Surface Temperature & Bioclimatic variables \\
Temporal frequency & Daily & Monthly \\
Temporal extent & $1-1-2009$ until $31-12-2015$ & $1-1-1970$ until 31-12-2000 \\
Spatial resolution & 250 meters & 1000 meters \\
Spatial extent & The Netherlands & Europe \\
& Latitude 50.75N - 53.55N, & Latitude 28.98 N- $73.22 \mathrm{~N} ;$ \\
& Longitude $3.35 \mathrm{~W}-7.22 \mathrm{E}$. & Longitude $16.30 \mathrm{~W}-48.25 \mathrm{E}$ \\
\hline
\end{tabular}

For our MAXENT model, bioclimatic data were used at a spatial resolution of 30 seconds $(\sim 1$ $\mathrm{km})$. Nineteen bioclimatic variables were downloaded from http:/www.worldclim.com. These variables are derived from monthly temperature and rainfall values in order to generate more biologically meaningful variables. In our study, we used six bioclimatic variables from the 2.0 Worldclim dataset (time series 1970-2000), being: BIO7 temperature annual range (maximal temperature of warmest month - minimal temperature of coldest month), BIO10 Mean Temperature of Warmest Quarter, BIO11 Mean Temperature of Coldest Quarter, BIO12 Annual Precipitation, BIO15 Precipitation Seasonality (Coefficient of Variation), and BIO18 Precipitation of Warmest Quarter. These variables were selected according to Cunze et al. (2016b). 


\section{Aedes albopictus occurrence}

Occurrence data for the MAXENT model was taken from Cunze et al. (2016a), and included occurrence locations of Ae. albopictus in Europe until 2015 from Kraemer et al. (2015b) and Koch et al. (2016). The 336 occurrence locations used in Cunze et al. (2016a) were verified and corrected according to the most recent occurrence knowledge (January 2018) on established populations in Europe from maps such as the ECDC map of Ae. albopictus (ECDC 2018c), EID Mediterranée maps of occurrence (www.moustiquetigre.org 2018), Collantes et al. (2015), and Prioteasa et al. (2015). Also new occurrence data was added using these maps. Data of interceptions of the species without confirmed establishment were excluded from the dataset. After verification, the occurrence data set accounted for 426 records in Europe.

For the LST-model, occurrence data of Ae. albopictus are not required. Instead, the suitability of the Netherlands is estimated based on temperature thresholds for Ae. albopictus survival and establishment.

\section{Land Surface Temperature model}

For this model we applied the workflow followed by Neteler et al. (2013) to turn the processed daily MODIS LST data into ecological indicators that can be used to assess the potential spatial distribution of Ae. albopictus. From this dataset, we derived the following predictors for the period between 2009 and 2015 in the Netherlands: mean temperature of January, the mean annual temperatures, and accumulated daily Growing Degree Days (GDD). These were first calculated per year. The overall probability averages were calculated based on the yearly probabilities between 2009 and 2015. The output indicators 1) probability of overwintering of eggs (POE), 2) probability of adult survival (PAS), and 3) probability of life cycle completion (PLC) were predicted. We adopted the methodology and the threshold values used by Neteler et al. (2013), based on the studies of Kobayashi et al. (2002), Roiz et al. (2011) and Caminade et al. (2012) as follow: (i) the threshold for the POE was set to $1^{0} \mathrm{C}$ for the mean January temperature with a margin of $2^{0} \mathrm{C}\left(-1^{0} \mathrm{C}-+3^{0} \mathrm{C}\right)$; (ii) the threshold for the PAS was set to $11{ }^{\circ} \mathrm{C}$ for the mean annual temperature with a margin of $2^{0} \mathrm{C}\left(+9^{0} \mathrm{C}-\right.$ $+13^{\circ} \mathrm{C}$ ); and (iii) the threshold for PLC based on 1,350 GDDs and $11^{\circ} \mathrm{C}$, was set to 1 st September, with a margin of one month. The GDDs are defined as the degrees exceeding a given threshold $\left(11^{0} \mathrm{C}\right.$ for Ae. albopictus) accumulated for all days in a given year (Kobayashi et al. 2002). Spatial data processing was performed with ArcGIS Pro. 


\section{Maximum entropy modelling: MAXENT}

The maximum entropy approach is one of the most commonly used prediction models to estimate potential spatial ranges of species, and it has recently been used for Aedes invasive species (Fischer et al. 2014, Melaun et al. 2015, Kraemer et al. 2015b, Koch et al. 2016, Cunze et al. 2016a, Cunze et al. 2016b). According to Baldwin (2009), the maximum entropy approach is relatively insensitive to spatial errors associated with location data, requires few locations to construct useful results, and performs better than other presence-only models. In our study, we used this approach implemented in the software MAXENT (v.3.4.1) (Phillips et al. 2004, Phillips et al. 2006) for the spatial extent of Europe (see Table 1). We used 50 replications for the model. From the 426 occurrence locations in Europe (see above), 419 were used (seven occurrence locations did not match with the environmental layers and were omitted).

The modelling performance was evaluated using the AUC-value (i.e. the area under the receiver operating characteristic curve value). This is a threshold independent measure that ranges between 0 (low performance) and 1 (high performance). The relative contributions of the six environmental variables to the MAXENT model were estimated using permutation importance estimation.

\section{Model validation}

The suitability maps obtained with MAXENT and LST-models were finally contrasted with the findings of Ae. albopictus during surveillance in used tire companies (2010 - 2015) to establish relationships considering the possibility of overwintering and survival of the species at these locations. The modelling results on predicted habitat suitability in both methodologies (value between 0 -1) were extracted for 11 occurrence locations at the used tire companies by a raster to point operation (QGIS v.2.18.14). Georeferenced data from surveillance of invasive mosquito species collected by the Centre for Monitoring of Vectors (CMV) of the Netherlands Food and Consumer Product Safety Authority (NVWA) was used for this assessment. This dataset included locations where the species had been found in used tire companies and where temperate strains of the species (the strain that could have potential for establishment in the Netherlands) were suspected to be introduced. Locations with interceptions of the species in greenhouses (indoors) with Lucky bamboo from southern China have not been used in the dataset. Due to the known origin of the imported plants, at these locations the tropical strain of Ae albopictus is suspected to be imported. This tropical 
strain does not produce diapausing eggs (Thomas et al. 2012), and would be unlikely to survive the cold winters outside the greenhouses (Takumi et al. 2009).

\section{Results and Discussion}

The findings of the invasive mosquito species Ae. albopictus in used tire companies in the Netherlands have demonstrated the long-distance human-aided dispersal capabilities of this species (Scholte et al. 2010a). Given its potential as a vector of infectious diseases, the question that arose was whether this exotic mosquito species could survive the climate conditions of the Netherlands and whether it would be able to build permanent populations in this country. The potential areas that may be suitable for Ae. albopictus populations in the Netherlands have been predicted in our study using two modelling approaches. For the LST-model, the habitat suitability in the Netherlands was estimated based on temperature thresholds for $A e$. albopictus survival and establishment. The MAXENT model instead predicted the habitat suitability in the Netherlands using current occurrence data of the species in Europe. We found marked differences in the predicted suitability values between the models.

For the LST-models, we used three variables (mean January temperature, mean annual temperature, and day of year when 1,350 GDDs are reached) as predictors to assess the habitat suitability of the Netherlands for Ae. albopictus. The overwintering ability of the diapausing eggs has been related to the January mean temperature in the study. Considering the averaged January mean temperature from 2009 to 2015, nearly all inland parts of the country express low suitability for diapausing eggs of Ae. albopictus (see Fig. 1-A, POE). The model predicts moderate suitability for egg overwintering in the areas along the major rivers in the Netherlands (Maas, Rijn, Ijssel, and Maas). The areas along the coast show a higher suitability for egg overwintering, especially the island of Texel and surroundings in the utmost north-western part of the country. In the model, adult survival is determined by mean annual temperatures, with $11^{\circ} \mathrm{C}$ as the lower limit for population establishment (Kobayashi et al. 2002). Results obtained with the temperatures from 2009 to 2015 show that the whole country is suitable for successful life cycle completion (see Fig. 1-C, PLC), and that suitable areas for adult survival are less restricted than the overwintering areas (see Fig.1-B, PAS). Given the predictor mean annual temperatures, highly suitable areas for survival of adult mosquitoes are the surroundings of the harbour of Rotterdam, the 
surroundings of Amsterdam, and large areas at the provinces of Limburg and Noord-Brabant, mostly associated to urban centres (Hertogenbosch, Eindhoven, Tilburg, or Maastricht).

Results obtained with the MAXENT model at European level accurately reflect the observed current distribution of the species. Modelling results show high probability of suitable conditions for Ae. albopictus in southern Europe, especially in the Mediterranean parts of Spain, France and Italy, and along the Adriatic shore (see Fig. 2). Our model depicts high suitability also in the western part of the Iberian Peninsula, the Rhone valley in France, and parts of the Rhine Valley in France, Switzerland and Germany. In the Netherlands, suitability values ranged from 0.06 in the western part of the country to 0.16 in the east. Compared to the predicted suitability in the areas in the Mediterranean coast (values of 1), the relative climatic suitability in the Netherlands can be considered to be very low. Using a replication of 50, the model accounted an AUC value of $0.947+/-0.02$ SD. According to the estimated permutation importance, our results show that winter temperature (temperature of the coldest quarter of a year) was the variable that contributed most to the model performance $(46.1 \%$; Table 2). The temperature annual range, and the precipitation and temperature in the summer also contributed to the performance of the model but to a lesser extent with $17.8 \%, 15.4 \%$, and $15.1 \%$ respectively (see Table 2 ).

Table 2. Permutation importance as a measure of relative contributions of the environmental variables to the MAXENT model for Aedes albopictus.

\begin{tabular}{llr}
\hline \hline Variable & & Permutation importance \\
\hline Bio11 & Mean Temperature of Coldest Quarter & 46.1 \\
Bio07 & Temperature Annual Range (BIO5-BIO6) & 17.8 \\
Bio18 & Precipitation of Warmest Quarter & 15.4 \\
Bio10 & Mean Temperature of Warmest Quarter & 15.1 \\
Bio15 & Precipitation Seasonality (Coefficient of Variation) & 3 \\
Bio12 & Annual Precipitation & 2.6 \\
\hline
\end{tabular}

\section{Model validation}

Suitability values calculated for the locations where Ae. albopictus has been observed are presented in Table 3 for the MAXENT model. Values at the Ae. albopictus findings in used tires companies in the Netherlands ranged from 0.07 in Moerdijk to 0.12 in Hardenberg. According to the MAXENT model results we assume that the winter temperatures in the Netherlands is the most limiting variable that constrains the survival of the diapausing eggs, thereby hampering establishment of the species. However, the species has been captured 
outdoors at used tire companies every year since 2010 at different locations across the country. These findings can be explained by the new introduction of used tires at the facilities containing Ae. albopictus diapausing eggs from areas where the species has established populations (e.g. southern France). If these tires are introduced after the coldest quarter of the year, summer temperatures will not limit egg hatching and larval development to adults.
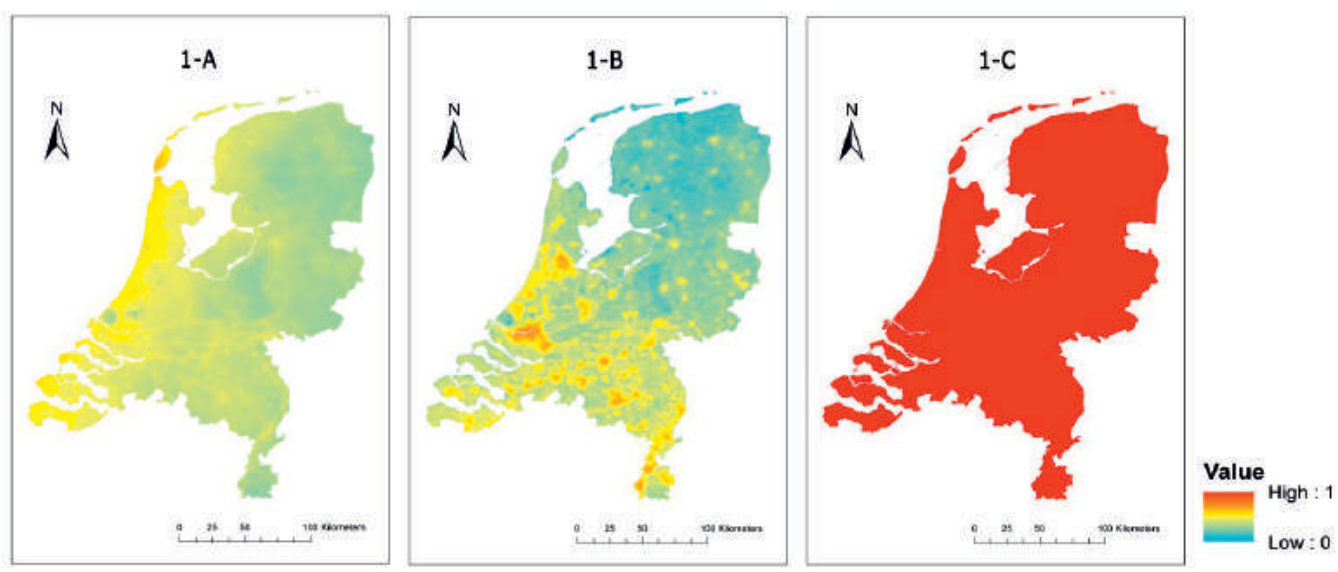

Fig 1. Results obtained with the LST-models for Aedes albopictus (years 2009-2015). 1-A. Probability Overwintering Eggs (POE); 1-B. Probability Adult Survival (PAS); 1-C. Probability life cycle completion (PLC). Map by authors.

Considering the average January temperature from 2009 to 2015 at the used tires locations in the LST model, Ae. albopictus does not encounter conditions that would completely limit the overwintering of diapausing eggs at these locations (see Table 3). For all locations where the species was found outdoors, the mean probability for overwintering of eggs ranged between $0.3-0.5$. Furthermore, when maps are considered per year rather than the 7-year average, January temperatures in 2012 and 2014 did not limit the overwintering of eggs at the locations where the species was found the previous year. On the contrary, overwintering of eggs is not considered likely in 2010, 2011 and 2013, and the presence of the species could be attributed to new introductions of specimens (eggs) at the tire importers in the next spring or summer. Furthermore, in 2014, annual temperatures also indicated a high probability for survival of adults at the positive locations (see Table 3). Considering the average temperature from 2009 to 2015, at the location Weert, the species also encounters suitable conditions for adult survival ( $\mathrm{P}=67 \%$, see Table 3). Using the same threshold, Ae. albopictus does not clearly encounter conditions for adult survival at several locations. Findings at locations in 
Emmeloord, Montfoort, Assen, Moerdijk, Lelystad and Almere accounted lower probability for adult survival $(\mathrm{P}<=40 \%)$. Furthermore, the annual temperatures in 2010, 2012 and 2013 also limited the conditions for survival of adults in several locations (see Table 3).

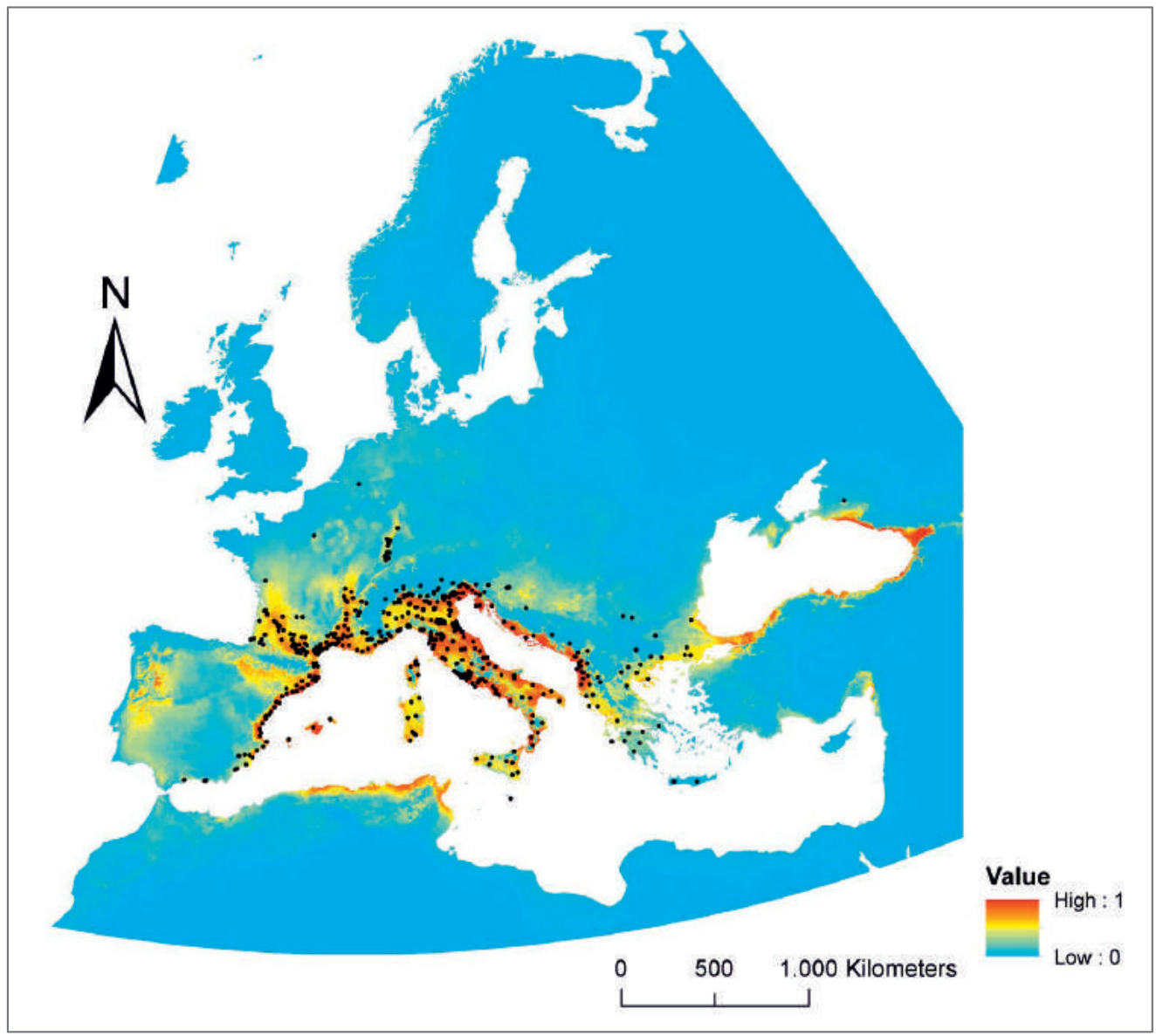

Fig 2. Observed distribution and modelled habitat suitability for Ae. albopictus with MAXENT. Black dots: occurrence records for Ae. albopictus used for the modelling. Map by authors.

\section{Implications for practice}

In the MAXENT model, the time period of the climatic data used for training (1970-2000) does not widely match the time period of the used occurrence data. First records of populations of the species in Europe (Italy) date back to the early 1990's, and for exam ple, records in southern Spain, or in northern locations in France were reported after the year 2000. Due to the proven invasiveness of Ae. albopictus, if the species would have been introduced before the year 2000 in these areas, we assume that it would have successfully 
become established. Unlike other similar models (Cunze et al. 2016a, Cunze et al. 2016b), our MAXENT model has only been fed with known current established populations. These studies, were performed using also occurrence data from interceptions without confirmation of establishment or overwintering (e.g. interceptions in Lucky bamboo greenhouses in the Netherlands). Results of these models could lead to an overestimation of the northern limits of the species in Europe.

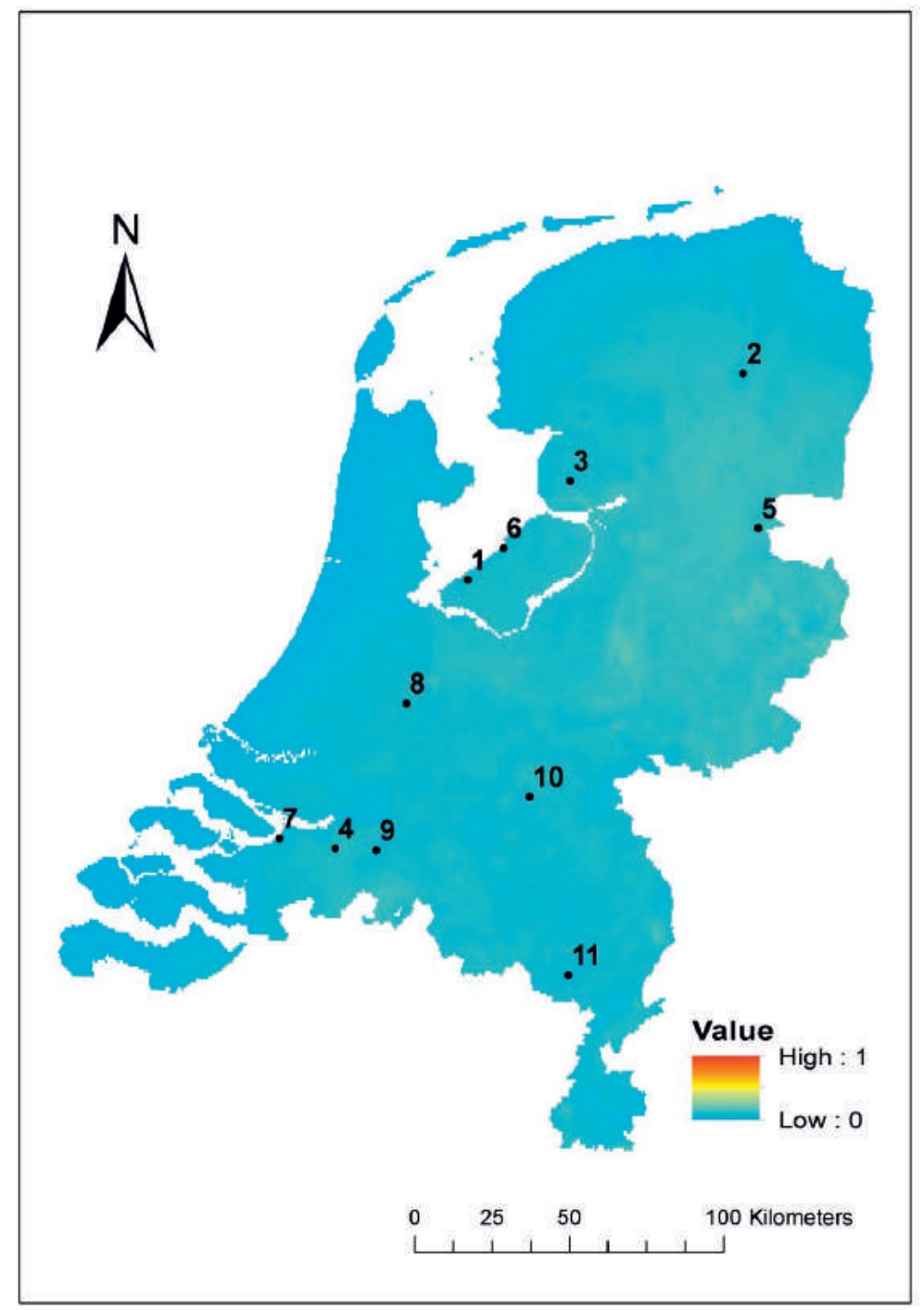

Fig 3. Habitat suitability for Ae. albopictus in the Netherlands with MAXENT model. Black dots: finding records of Ae. albopictus. Locations: 1. Almere, 2. Assen, 3. Emmeloord, 4. Etten-Leur, 5. Hardenberg, 6. Lelystad, 7. Moerdijk, 8. Montfoort, 9. Oosterhout, 10. Oss, 11. Weert. Map by authors. 
As observed in previous species distribution models (Caminade et al. 2012, Fischer et al. 2014, Cunze et al. 2016a), an expansion of climatically suitable habitats in western and central Europe is expected in the near future for Ae. albopictus. In our study, climate change scenarios have not been taken into account and only current climate data have been studied. As known from the climate scenarios developed by the Royal Netherlands Meteorological Institute (KNMI), even under conservative and optimistic scenarios, the ambient temperature in the Netherlands is expected to continue to rise, with an increase of values between $1^{0} \mathrm{C}$ and $2.3^{\circ} \mathrm{C}$ in 2050 , and $1.3^{\circ} \mathrm{C}$ and $3.7^{\circ} \mathrm{C}$ in 2085 (KNMI 2014). That means that climate change will undoubtedly increase the winter temperatures, leading to the increase of the probability for overwintering of eggs, and consequently increasing the risk of establishment of $A e$. albopictus in some parts of the Netherlands within 30 years.

The lowest suitability values of the LST-model corresponded to forested areas and greenhouse farming houses, and the highest probability values were found in urban areas (e.g. built-up areas, residential, industrial). Our findings reflect the results published by (Guo et al. 2012) who found that built-up areas with paved roads, residential, and factory buildings have a higher LST, and vegetated land covers have the lowest LST. Possible explanation for a low LST in vegetated areas (e.g. forests) is that the LST is collected from the forest canopy, which is influenced by the cooling effect of evaporation. The low suitability accounted in the greenhouse buildings situated in the western part of the country, is directly related to the highly reflective roofs of the greenhouses, an artefact which suggests that these locations are cool places.

The results of our study give a new perspective on the previous study of Takumi et al. (2009). The latter study was based on climatological information from weather stations (only from year 2006), and concluded that winter conditions in the Netherlands were permissive for the establishment of temperate strains of Ae. albopictus. In our study, two parameters that act against the establishment of Ae. albopictus in areas where diapausing eggs may overwinter, were also taken into account: the annual mean temperature $\left(11^{\circ} \mathrm{C}\right)$, and the day of year when 1,350 GDDs are reached. These parameters are considered important because they affect the time needed for Ae. albopictus larvae to mature into adult mosquitoes during the period of seasonal activity. It should be mentioned that in comparison with the study of Takumi et al. (2009), we did not use precipitation data in the model. For optimal development of $A e$. albopictus, the mosquito species is known to require at least $500 \mathrm{~mm}$ of annual rainfall (Medlock et al. 2015). The mean annual rainfall in the Netherlands ranges from about 700 to 
$900 \mathrm{~mm}$ (KNMI 2018), and is not considered a limiting factor for the species in the study area.

In our study, the results depend on the selected thresholds for the January temperature $\left(1^{0} \mathrm{C}\right)$, annual temperature $\left(11^{\circ} \mathrm{C}\right)$, and day of year when 1,350 GDDs are reached. These threshold values were based on Kobayashi et al. (2002), Roiz et al. (2011) and Caminade et al. (2012). Modifying these temperature thresholds with a few degrees could result in a substantial change in the suitable area for the species. For example, lowering the January and annual temperature thresholds a few degrees would increase the area regarded as suitable for $A e$. albopictus. In the same way, moving by several weeks before the threshold value when 1,350 GDDs are reached may result in a decrease in the area regarded as suitable for the Asian tiger mosquito.

In the Netherlands, after the implementation of a risk-based surveillance strategy on invasive mosquitoes, Ae. albopictus has been intercepted yearly at used tire companies or its surroundings (see Chapter 6 in this thesis). However, the species has not successfully established in these interception areas. Because surveillance and control on invasive mosquitoes started in 2010, and used tire companies had already imported tires stored outside before the start of the surveillance, Ae. albopictus may easily have been introduced many years before 2010. The question is, in the absence of surveillance and control measures, what factors could have worked against the successful establishment at these areas? Are low temperatures indeed lowering the probability of suitable conditions? The MAXENT results for example indicated low suitability for the species in the Netherlands, and only one location (Moerdijk) in the LST-model results showed moderately suitability for overwintering of eggs (value $<0.5$ ). The length of the reproductive season in the Netherlands could also contribute to lowering the probability of suitable conditions. This length is in fact considerably shorter in the Netherlands than at southern latitudes in Europe, due to differences in temperature mainly. That means that the temperature necessary to stimulate the hatching of diapausing eggs will occur later in the season in the Netherlands than in southern regions, and the temperature causing adult mortality will arrive early in the season. However, the Asian tiger mosquito is a species with a high ecological plasticity that can cope with a wide range of conditions, has competitive ability, and has demonstrated to have the potential to adapt fast to local habitat conditions during the invasion process (Paupy et al. 2009). All these facts might allow Ae. albopictus to adapt to colder temperatures in the new invaded locations, facilitating faster establishment in colder regions. 


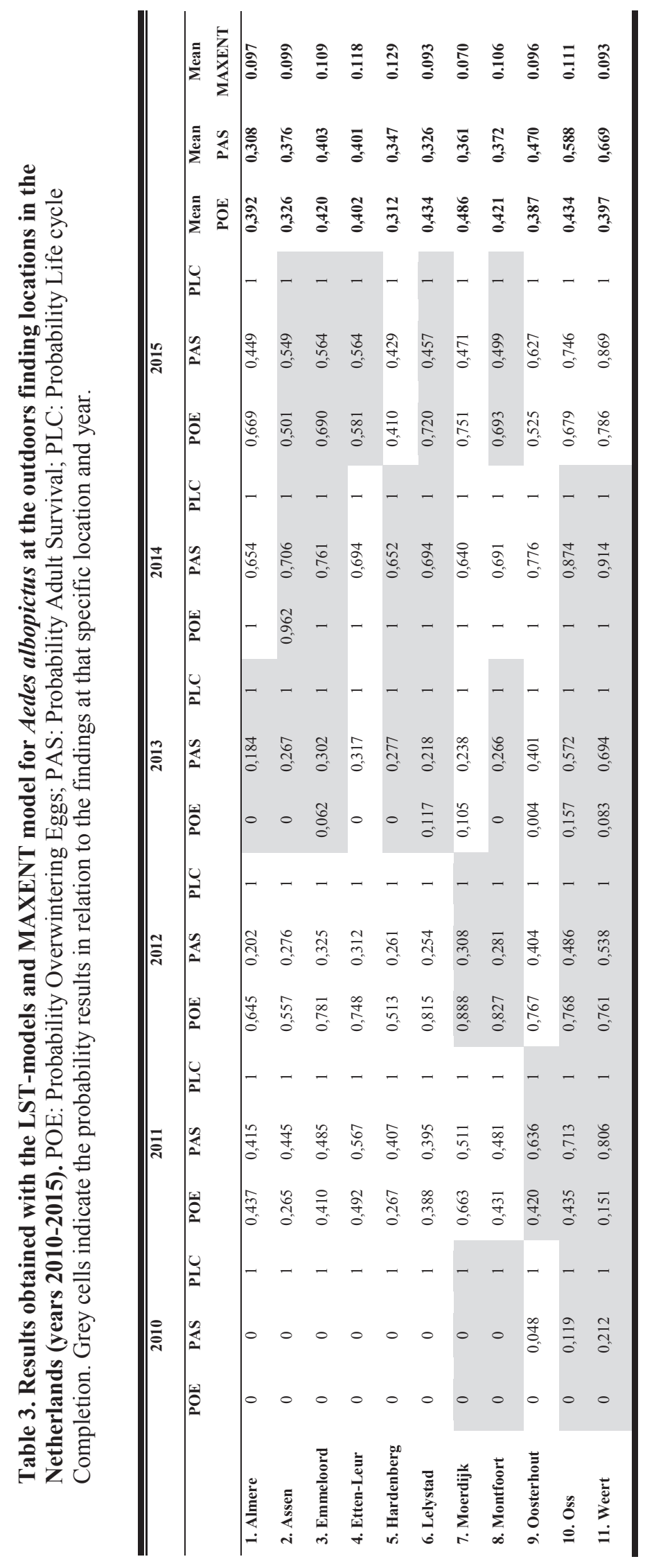




\section{Future recommendations}

Our study has been conducted using climatic variables exclusively as the main factors affecting the distribution of Ae. albopictus in the Netherlands. However, other factors such as modification of land cover/land use, habitat availability and microclimate can influence the success of the establishment in an area significantly. In the case of the Netherlands, where the low winter and annual temperatures are the limiting factors in the model, hibernation of diapausing eggs indoors, or in warm and protected areas in cities, could protect the mosquito from cold events and may be responsible for local establishments under these special conditions. Especially the effect on the microclimate created by the tires could not be assessed within the framework of this study but is potentially influential. As also shown in our results, especially around cities higher temperatures can be expected (heat island effect) (Zhao et al. 2014), and potential breeding sites such as urban catch basins can provide favourable microclimate conditions for overwintering of diapausing eggs compared to more cold-exposed sites (Ravasi et al. 2018). Unfortunately, these are factors that could not be considered in this study and are recommended for future investigations. Because the species is currently invading areas northwards in Europe (ECDC 2018c), regular updates of the modelling using updated occurrence and climate data are recommended.

The results obtained lead to a better understanding of the species' potential distribution, and identified areas in the Netherlands with a risk for the establishment of the species. However, the final results of the two modelling approaches differed considerably, and for the interpretation of these results, one should need to be aware of the limitations of both modelling approaches. The MAXENT model, based on the current occurrence of established populations in Europe, indicates that in comparison with areas in southern regions in Europe, the area of the Netherlands show low suitability for populations of Ae. albopictus. However, the LST-model, based on the temperature thresholds for the species, by using current LST data, shows that the species can always complete its life cycle successfully, and in years with mild winter conditions and warm summer, diapausing eggs can survive and develop to adults. The results obtained in the study seem to be appropriate to help to optimize the design of the monitoring and control activities with the final aim of preventing establishment and avoid further spread of this vector of human diseases. If the species is found introduced, special attention should also be taken in urban and peri-urban areas, where the species inhabit using artificial containers and catch basins as breeding sites. The variability of microclimate among sites in the complex urban conditions could provide a more favourable microclimate 
conditions for the species, and thus those areas should be placed under intensive surveillance. Experiences from the invasion in Europe tell us that once this species has colonized an area, eradication might be difficult or impossible to achieve (Paupy et al. 2009, Baldacchino et al. 2015).

\section{Conclusions}

This study provides suitability maps for Ae. albopictus with particular reference to the Netherlands using the state-of-the-art on occurrence and environmental data. Results obtained using LST and MAXENT models for predicting the suitability of the Netherlands for the Asian tiger mosquito are different, with the MAXENT model the more restrictive for the species. Based on the LST-model, the current climatic conditions hamper the successful overwintering of eggs of Ae. albopictus and their survival as adults in many areas of the country, but in warm years with mild winters, several parts of the Netherlands offer climatic conditions suitable for developing populations. Our results for the year 2014 suggest that such situations do occur, including at locations of used tire companies where the species has been found outdoors. For this reason, risk-based surveillance is crucial for promptly detecting Ae. albopictus at risk locations and engage mosquito control actions.

\section{Acknowledgements}

We would like to thank Dr. Nienke Hartemink, Mr. Arjan Stroo and Mr. Anton van der Sommen for critically reviewing the manuscript and the colleagues of the Netherlands Food and Consumer Product Safety Authority (NVWA) for assistance collecting the data on which the model validation is based.

\section{Abbreviations:}

CMV: Centre for Monitoring of Vectors

IMS: Invasive Mosquito Species

LST: Land Surface Temperature

MAXENT: Maximum Entropy approach 
MODIS: Moderate Resolution Imaging Spectroradiometer

SDM: Spatial Distribution Models

VBD: Vector-Borne Diseases 

Chapter 10

General discussion 
This thesis examined the spatial distribution of mosquitoes that can be vectors of infectious disease in the Netherlands, as well as the risk of introduction and establishment of invasive mosquito species in the country. In the recent decade, several exotic mosquito species have become established in European countries, and further expansion of their distribution into the Netherlands seemed likely. The reason for the study was the increasingly frequent reporting of invasive mosquito species (IMS) in the country, as well as the lack of detailed knowledge on the spatio-temporal distribution of the mosquito fauna. In this thesis I analyzed and evaluated results of a surveillance of mosquito species in the Netherlands, conducted from 2010 by the Centre for Monitoring of Vectors (CMV) of the Netherlands Food and Consumer Product Safety Authority (NVWA). Central to my work are the investigation of the current and potential spatial distribution of indigenous and exotic mosquito species, and a critical evaluation of the surveillance and control methodologies applied. Accurate information on the mosquito species responsible for transmission of vector-borne diseases (VBD) and their spatial distribution is essential for identification of locations at risk for VBD transmission.

At the start of the study, a survey was conducted to establish a baseline for the spatiotemporal distribution of the indigenous mosquito fauna present in the Netherlands (Chapter 2). This survey, conducted from 2010-2013, provided occurrence maps for 26 indigenous species, and one invasive mosquito species (Aedes japonicus). Furthermore, the habitat preferences, diversity and phenology of the species were also analyzed in the study. Using the data on occurrence and abundance from Chapter 2, a special study on the potential spatial distribution and population density of Anopheles plumbeus, a native nuisance mosquito species, was done to confirm reports that this species was increasing in density as well as in geographic distribution (Chapter 3). I found a high environmental suitability and potential abundance of this species in the south-eastern provinces of Limburg and North Brabant. These areas, mostly associated with abandoned farm buildings, coincide with the areas where in recent years, most nuisance has been reported. Where An. plumbeus is found, it can be a serious nuisance through its aggressive human biting behaviour. In Chapter 4, the current knowledge of pathways associated with the introduction and dispersal of IMS in Europe was reviewed. Four main routes for IMS introduction and dispersal were identified and discussed: the trade in used tires, the import of Lucky bamboo plants from Asia, the passive transport of IMS in vehicles (traffic by road, airplanes, and sea), and the natural dispersal of IMS. Here I found that the trade in used tires remains the main pathway for long distance transportation of IMS into and within Europe, and that passive transport in ground vehicles represented the 
major driving force for dispersion from already established IMS populations. Next, in Chapter 5, previously undocumented pathways of IMS introduction were investigated. This study was also intended to identify m osquito experts' perceptions regarding control m easures against IMS introduction via different pathways. Results showed that ground transportation (with cars, trucks, etc.), passive natural dispersal, and used-tire shipments are perceived as the most likely pathways for introduction and dispersal of IMS in Europe. I also demonstrated that the perceived efficacy of surveillance and control measures is key for the experts to overcome constraints and to support the implementation of actions against IMS introductions. In Chapter 6, I analyse the results of the risk-based and adaptive surveillance implemented at Lucky bamboo and used tire importers to prevent the establishment of IMS in the Netherlands. Results show that imported Lucky bamboo shipments carried Ae. albopictus every year since 2010, and for the first time, Ae. japonicus was associated with the import of this plant species as well. At used tire companies, IMS identified were Ae. albopictus, Ae. atropalpus, Ae. aegypti, and Ae. japonicus, of which Ae. albopictus has been found each year since 2010. The study concludes that implemented risk-based and adaptive surveillance is an effective methodology for the detection of IMS, and the application of governmental management measures in combination with mosquito control has kept introductions under control at these locations in the Netherlands. In Chapter 7, results of surveillance conducted at the international airport of Schiphol are presented. This surveillance focused on promptly detecting accidental introductions of IMS at the airport facilities in order to quickly react and avoid temporary proliferation or establishment of mosquito populations, identify the origin of the introductions, and prevent potential transmission of vector-borne diseases. In the study, I found Ae. aegypti mosquitoes at different locations at the airport, presumably transported in containers transported within aircraft storage compartments. GIS analysis and genetic assignment tests on these mosquitoes pointed to North America or the Middle East as possible regions of origin. The effectiveness of mosquito control on reducing populations of an established IMS (Ae.japonicus) in the Netherlands was studied in Chapter 8. The study was designed to understand the dynamics of Ae. japonicus populations after the application of targeted mosquito control at breeding hotspots of the species: allotment gardens. Results show that mosquito control actions resulted in a significant reduction in Ae. japonicus larval abundance. Results also show that rain barrels are the most frequent breeding sites of this species at the allotments. Due to frequent findings of Ae. albopictus during routine exotic mosquito species surveillance at companies that import used tires, the potential risk for establishment at these locations in the Netherlands was studied using two habitat suitability 
modeling methodologies (Chapter 9). First, a species distribution model based on the maximum entropy niche modelling approach (MAXENT) taking into consideration the most updated occurrence data of the species in Europe, and second, a spatial logic conditional model based on the temperature requirements of the species and using Land Surface Temperature data (LST-model). Results obtained showed differences in the predicted suitability of the Netherlands for the species, with the MAXENT model predicting a more restrictive distribution for the species. Instead, based on the LST-model, the current average climatic conditions limit the overwintering of Ae. albopictus eggs and adult survival in only selected inland areas of the country, and the entire country is suitable for successful life cycle completion if the species is introduced after the winter months.

The methods and approach used for obtaining the knowledge described in this thesis have resulted in accurate occurrence maps of indigenous mosquito species and detections of IMS introductions at high risk locations. This is pivotal for the evaluation of intervention strategies for the prevention of mosquito-borne disease outbreaks. In this General Discussion, I will discuss the implications of my findings, putting them in perspective for future challenges on acquiring knowledge about the spatial distribution of mosquito vectors of infectious diseases.

\section{Surveillance of mosquito species}

Spatial distribution of mosquito vectors of diseases tends to be heterogeneous, thus not uniformly distributed within their potential range. Populations of mosquitoes are usually patchwise due to factors such as habitat characteristics, environmental factors or the presence of hosts. Understanding the spatial preferences of mosquito species contributes to the identification of locations at risk of mosquito-borne diseases. As the presence of the mosquito vector is a basic requirement for pathogen transmission to occur, if environmental conditions for transmission are suitable, the higher the abundance of pathogen-infected vectors, the higher the risk of disease transmission. Surveillance is defined by the European Centre for Disease Prevention (ECDC) as a set of procedures implemented in response to a recognized risk (ECDC 2012). The implementation of surveillance for the presence of native and invasive mosquitoes is crucial to gain information on the spatial distribution of mosquito species in a given area. Therefore knowledge on the presence and distribution of mosquitoes needs to be regularly updated by implementing systematic and harmonized surveillance procedures across Europe (ECDC 2014a). The implementation of a surveillance of native 
mosquito species in Chapter 2 depicts for the first time the spatio-temporal distribution of the mosquito fauna in the Netherlands. Except for the studies in the MODIRISK project (Versteirt et al. 2009), prior information about mosquito species distribution was missing, and only species composition and phenology traits were available for a limited number of sites (Verdonschot 2002). Mapping the distribution of mosquito species using the strategy applied in Chapter 2 is strongly recommended as a routine surveillance for native mosquitoes. The number of species found and expected, information on mosquito diversity, and the geographical distribution of the species obtained by using this method, suggest that this survey approach is efficient. Further longitudinal surveys (a design that involves repeated sampling over periods of time) as carried out by CMV, will also provide more insight in the abundance over time of the different mosquito species at specific locations. Occurrence and abundance data obtained using this surveillance strategy are also useful in spatial distribution models (SDM) (Cianci et al. 2015), as shown in Chapter 3 for the nuisance and potential malaria vector An. plumbeus. Furthermore, in addition to the indigenous species captured during the survey, individuals of the IMS Ae. japonicus were detected using this strategy (Ibañez-Justicia et al. 2014). This strategy is well suited to not only better understand the distribution of the indigenous mosquito fauna but also as a passive technique for detection of hitherto unknown populations of established IMS. Due to expected changes in climate and its influence on ectothermic arthropods such as mosquitoes, and possible changes in land use and socioeconomic conditions, continuation of these extensive studies is recommended to determine temporal differences in occurrence of abundance.

In order to obtain additional information to update knowledge on the occurrence and distribution of mosquito species, citizen science projects can be implemented (Kampen et al. 2015). Citizen science has recently been used as an important data collection source in many scientific disciplines (Gura 2013) and is based on the enrollment of interested citizens to obtain scientific data at low costs. As active mosquito collection is usually cost-, time- and labor-intensive and can cover only small parts of a country, passive data collection approaches are gradually being integrated into monitoring programmes in several EU member states (Kampen et al. 2015, Vogels et al. 2015). In Germany, the citizen science project 'Mueckenatlas', conducted since 2012 and financed by the German Federal Ministry of Food and Agriculture (BMEI), is considered as an efficient alternative to routine active mosquito surveillance, covering a similar species spectrum and distribution as in trapping. In addition, this methodology has been proven effective to detect incursions of IMS in Germany 
such as Ae. aegypti, Ae. albopictus, Ae. japonicus and Ae. koreicus (Kampen et al. 2012, Werner and Kampen 2015, Kampen et al. 2016, Werner et al. 2016). While passive surveillance has been proven to provide occurrence data over large areas, active surveillance by trapping provides higher quality data, such as presence and absence data, abundance, seasonal activity, and samples for pathogen screening (Kampen et al. 2015, Jordan et al. 2017).

The probability for early detection of an IMS or for rapid interruption of transmission once an outbreak of a mosquito-borne disease occurs, is directly related to an adequate surveillance system (Vazquez-Prokopec et al. 2010). Apart from gaining information on the spatial distribution of mosquito species in a given area, risk-based surveillance of IMS is key to promptly detect introductions at risk locations (e.g. Lucky bamboo greenhouses, used-tire traders, or points of entry such as airports), allowing appropriate and rapid implementation of control measures. In order to be effective in the detection of IMS, risk-based surveillance needs to consider all known likely introduction pathways for IMS. As shown in Chapter 5, information over the main pathways of introduction is well documented in the literature, and known by the European mosquito experts. Furthermore, results of the risk-based surveillance implemented in Chapters 6, 7 and 8, confirm that the Netherlands is exposed to introduction of IMS. The main findings of the IMS surveillance are: i) since 2010 Ae. albopictus has been intercepted every year in both used-tire and Lucky bamboo companies, ii) discovery of an established population of the IMS species Ae. japonicus in the municipality of Lelystad, iii) the first evidence of Ae. japonicus imported with used tires in the Netherlands and in Lucky bamboo elsewhere, and iv) the first evidence in Europe of airline transport of Ae. aegypti. The fact that IMS are introduced and are able to build up populations, together with the global emergence of MBD, emphasizes the necessity for implementation of IMS surveillance to detect introductions and avoid their establishment. As shown in the results of Chapter 6, early stage detections of IMS introductions will allow effective implementation of control measures, and consequently contribute to preventing MBD.

The surveillance results presented in this thesis have been obtained from the implementation of a defined strategy (e.g. trap type, frequency sampling, etc). The election of a different strategy, as for example, use of other trap types, would probably have affected the obtained results in terms of occurrence of mosquito species and/or the measured abundance. As recommended by the World Health Organisation (WHO), collaboration between mosquito monitoring teams in Europe needs to be strengthened with the aim of standardizing the 
collection of mosquito surveillance data (WHO 2017). This standardization will contribute to the harmonization of data and allow for comparison of results at the European level.

\section{Current and future climate affecting spatial distribution of mosquito} species

As ectothermic arthropods, mosquitoes are unable to regulate their body temperature. As a consequence, thermal conditions directly regulate important processes such as the rate of immature development, host biting rate, blood digestion, and egg production. In the same way, as an obligate aquatic insect at the immature stadium, the survival and abundance of immatures depend on the availability of water. The alteration of the environmental conditions due to climate change will undoubtedly have consequences for mosquito populations, and as a consequence, public health will probably be adversely impacted (Patz et al. 2014, Semenza and Suk 2018).

Future climate predictions for the Netherlands indicate an increase in precipitation in all seasons except the summer, with an increased likelihood of extreme rain showers, thunderstorms and hail in summertime (KNMI 2014). The latter events will affect the mosquito populations by increasing the availability of breeding sites. Man-made container breeding mosquito species, and especially IMS, will benefit from these breeding sites. Even under optimistic and conservative climate change scenarios, future projections indicate an increase in air surface temperatures and changes in the hydrological cycle that may affect the geographical distribution and breeding seasons of the insect vectors that transmit vectorborne diseases (Proestos et al. 2015). In the Netherlands, the ambient temperature is expected to continue to rise, with increases between $1{ }^{\circ} \mathrm{C}$ and $2.3^{\circ} \mathrm{C}$ in 2050 , and $1.3^{\circ} \mathrm{C}$ and $3.7^{\circ} \mathrm{C}$ in 2085, depending on the scenario (KNMI 2014). The number of cold winter days will decrease, and the number of warm summer days will increase, as will the likelihood of heat waves. These climatic changes may support a geographic shift of native mosquito species populations, a change in their seasonal dynamics, and a further increase in the probability of regional establishment or spread of IMS beyond the current boundaries (Schaffner et al. 2013).

As temperature-dependent species, the predicted general increase of temperatures will affect seasonal dynamics by lengthening the activity period of mosquitoes. Adults will appear earlier in the spring and they will disappear later in the autumn. With fewer cold winter days, 
the activity of mosquitoes that hibernate as adults will increase. Species as Cx. pipiens, which hibernate as adult in the Netherlands, could be more active during the winter months causing nuisance (Vogels et al. 2015). With the improved overwintering success due to increasing temperatures, the probability of VBD transmission will increase consequently. Overwintering behaviour is a vital aspect of disease transmission because diapausing mosquitoes may act as a pathogen reservoir between seasons when reservoir hosts are no longer infectious (Swellengrebel et al. 1938, Medlock and Vaux 2015). Fewer cold winter days will also have a direct effect on IMS's such as Ae. albopictus. Fewer cold days will increase the probability of egg overwintering (see Chapter 9), thus increasing the chance of establishment of this species in the country. The increase of warm summer days and the expected heat waves will probably have an important effect on mosquito abundance and survival. An increase of temperatures in the summers will increase peak mosquito abundance (Ewing et al. 2016). This will be observed especially in late summers, when temperatures peak in mid-to-late August and lead to a higher mosquito abundance compared to early summers when the mosquito population increases more slowly (Ewing et al. 2016). This increase in vector abundance in short periods due to climate change will potentially increase mosquito nuisance and the risk of VBD transmission in the Netherlands. Furthermore, an increase in the number of warm summer days will increase the number of generations that important vectors such as Ae. albopictus or Ae. aegypti can complete, and increase the risk of VBD outbreaks transmitted by these species.

\section{Effect of socioeconomic and human changes on the distribution of mosquitoes}

Distribution and abundance of mosquito vectors of disease can be affected by changes in socioeconomic and human activities. Land-cover change, such as deforestation, reforestation, or fragmentation of habitats can affect the mosquito populations. The landscape structure of the Netherlands is characterized by high fragmentation and even in small areas, urban and rural habitats are adjacent and connected to natural habitats. Such fragmentation puts vectors, wildlife, domestic animals, humans, and pathogens in contact with each other. Special attention is required in areas where host species arrive from areas where VBDs are endemic (e.g. migrating birds from WNV endemic areas). Therefore, these areas should be of prime concern in disease risk assessment. Furthermore, an increase of natural areas with water 
bodies (e.g. wetlands, flooding areas of rivers) can influence the presence and abundance of mosquito vectors. These changes can alter the occurrence of mosquito breeding sites, mosquito shelter, and the interactions and abundance of wildlife and domestic hosts. The change of landscape features can also affect the presence and abundance of vectors. As an example, the diminishing of populations of the malaria vector Anopheles atroparvus and the eradication of malaria in northwestern Europe was related to hydrological measures such as heightened dikes, larger canals and more effective sluices, that reduced salt-water leakage and inundation risks, and therefore eliminated the natural habitat of the species (Knottnerus 2002).

Economic conditions may also affect mosquito presence and abundance. Poverty in city suburbs or long-term uncontrolled settlements of refugees could affect the presence of breeding sites and proliferation of mosquito vector populations due to the uncontrolled accumulation of water. This fact in combination with the possible introduction of diseases from endemic areas can pose a risk in these areas. On the other hand, wealth can also affect the presence and abundance of mosquitoes. Wealth is often associated with an increase in outdoor recreational activities. As demonstrated in the Netherlands in Chapter 8 in this thesis, and in Germany (Becker et al. 2017), recreational allotment gardens in peri-urban areas can be prolific for indigenous and invasive mosquitoes due to the abundance of manmade breeding sites. Furthermore, the increase of people moving permanently to rural areas (restoration of old farms for permanent residence) and the corresponding increase in outdoor recreational activities associated with a wealthy lifestyle increases exposure to mosquito bites, and the risk of contact with zoonotic pathogens. Increase in wealth also makes travel more accessible to people, and consequently increases the frequency of disease vector movement inside vehicles, as demonstrated in aircrafts in the Netherlands (Scholte et al. 2014) (Chapter 7). In addition, it would increase the likelihood of people bringing foreign pathogens that can then be transmitted locally. In the same way, the improvement in quality of European highways and the increased autonomy of new private vehicles can nowadays increase long distance dispersal of IMS from infested areas. Traveling long distances by car with the air conditioning on and car windows closed, together with extensive holiday travel in motorhomes can contribute to increase of hitchhiking IMS in Europe.

Changes in economic activities can also affect the presence and abundance of disease vectors. The abandonment of activities such as pig farming, leaving the pig stables empty, has been related to the local proliferation of the potential malaria vector An. plumbeus due to the 
presence of breeding sites associated with water filled manure pits (Schaffner et al. 2012, Heym et al. 2017). This situation causes nuisance to the humans living in the surroundings, and eventually could lead to autochthonous malaria cases (Kruger et al. 2001), created by the chance of putting people carrying malaria parasites into contact with a high density of this malaria vector. Therefore, surveillance and implementation of control measures should be considered in disused farms if public health issues appear related to An. plumbeus.

Market prices and future market trends also can alter the pattern of IMS introduction, especially with IMS transported from abroad. As an example, the increase of prices for new tires can increase the volume of accumulated used tires at importing companies due to the expected demand. This can lead to the increase of the risk of movement of IMS from abroad. The trade in Lucky bamboo plants from Asia has introduced the Asian tiger mosquito in the USA (Madon et al. 2002) and in Europe (Scholte et al. 2007, Demeulemeester et al. 2014). Convincing people to choose a non-egg-carrying alternative to Lucky bamboo or sourcing the plant from areas free of Ae. albopictus could drastically reduce introductions of this species into Europe. In addition, a factor that may affect the market for these products is the occurence of a financial crisis. In this case, reduction of economic resources due to budget cutting measures, can compromise the prevention, monitoring and control actions on vectors of diseases.

\section{Accuracy of habitat suitability models}

Knowledge about the potential spatial distribution of mosquito species can be acquired using models. Species distribution models estimate the suitability of the environment in unsampled areas for a species, based on the known occurrence of the species and related to environmental variables. For this reason, long term data collection (over several successive years) in a study area is important for obtaining occurrence data for the mosquito species used in the models. Data collection conducted over a short term period, even if conducted extensively thoughout the entire study area, can lead to false conclusions regarding the presence of a species. This is especially important if the mosquito sampling period does not correspond to the season when the species normally occurs, or if the presence of a mosquito species is closely tied to following special environmental events such as river flooding or a temporal increase of saline or brackish coastal water bodies. In these cases, these events will provide larval temporary development sites for floodwater and salinity-tolerant mosquito 
species not otherwise available (Ramasamy and Surendran 2012). As conducted in this thesis, sampling the study area during several seasons within in one year provided accurate information about the spatiotemporal occurrence of the mosquito species and, at the same time, prevented false species absence records.

One of the factors that affect the performance of the presence/absence SDM is the sampling methodology chosen for obtaining the occurrence of the species studied (e.g. larval/pupal sampling, adult trapping, etc). Georeferenced data of species presence in the surroundings of the natural breeding sites would ideally provide the best information to correlate the existing environmental characteristics with potential occurrence. As shown in Chapter 3 for the indigenous mosquito species An. plumbeus, high quality data obtained from mosquito surveillance was required to estimate the potential occurrence and abundance of the species. In this case, the occurrence of the species was highly correlated with the selected environmental variables. However, for other mosquito species of importance in VBD transmission, even with high quality data obtained from targeted and unbiased mosquito surveillance as used in Chapter 2, the potential occurrence and abundance is unlikely to be estimated with certainty as species ubiquity can be a factor influencing the final prediction of the models. For ubiquitous mosquito species that can occupy different types of habitats and breeding sites, presence/absence based models may be less useful for predicting areas unsuitable for the species. In other words, the specificity (ability to predict absence sites) will be compromised for ubiquitous species. For mosquito species such as $C x$. pipiens, which is the most ubiquitous mosquito species in temperate regions occurring in rural and domestic environments (Farajollahi et al. 2011), models predicting their presence or absence are relatively unreliable as conditions for its absence are very few. Problems with modelling can also be identified for mosquito species with a long flight capacity. In this case, host-seeking females can be found using $\mathrm{CO}_{2}$ baited mosquito traps over long distances in any direction. For mosquito species such as Ae. vexans, a species recently found to be a competent laboratory vector of Zika (O'Donnell et al. 2017) and Rift Valley fever (Turell et al. 2010), data obtained by trapping may not be appropriate due to the large distances that the hostseeking females can fly on their way to find a host (Briegel et al. 2001). In this case, hostseeking behaviour can confound possible correlations with the environmental variables, and suggest a distribution biased towards the randomized availability of blood hosts.

Data obtained with the sampling methodology described in Chapter 2, has proven to be useful for verification of spatial distribution models that predict the probability of occurrence 
of mosquito species based on presence/absence data (see Chapter 3). Nevertheless, prediction of abundance of these species using this data is far from accurate. Due to the limitations of the applied mosquito sampling methodology (only one sample collected at each sampling site in a period of one week), the maximum number of individuals that potentially could be present at sampling sites, and their temporal variation, could not be accurately assessed as shown in Chapter 3. Without accurate predictions of the vector density, calculations of the basic reproduction number of the disease (R0), which describes the ability of a disease to persist in a susceptible population of hosts, will be subject to considerable error (Ewing et al. 2016). The probability of occurrence of a species in a study area can be a good indicator of the potential relative abundance of the species, but in case of MBD outbreaks, accurate values regarding the abundance of vector species are essential for risk assessments. In this case, longitudinal mosquito sampling, obtaining frequent records (weekly, bi-weekly) of the number of individuals at several locations and across several seasons, can provide this information. For example, by analyzing a highly detailed longitudinal dataset of the abundance and spatial distribution of WNV vectors, accurate predictions have been made regarding their spatial occurrence, abundance peaks, and potential areas suitable for the introduction and amplification of WNV in northern Italy (Bisanzio et al. 2011). Generating quality data for abundance modelling is one of the largest challenges for mosquito surveillance. A consensus for a sampling methodology allowing combining and comparing results among European teams (Mohlmann et al. 2017) is therefore highly recommended.

Results obtained with SDM help to understand the potential distribution range of the species studied in a defined area. However, the spatial resolution of large-scale distribution models may not be sufficient to detect particular climatic conditions existing for example in urban settings. The special climatic conditions in urban centres can create favourable conditions for the possibility of establishment of IMS. Especially around large cities higher temperatures are expected due to a heat island effect (Zhao et al. 2014), and urban breeding sites can provide favourable microclimate conditions for overwintering of IMS diapausing eggs compared to more cold-exposed sites. As shown in Switzerland (Ravasi et al. 2018), large-scale models of climatic suitability for Ae. albopictus do not take into account conditions of urban microhabitats, such as catch basins, that can provide unforeseen conditions for the survival of eggs of Ae. albopictus. The findings of the Swiss study confirmed previous conclusions (Vallorani et al. 2015) that winter temperatures in catch basins are higher and more stable 
compared to external temperatures. This fact illustrates the importance of using high resolution climate data in models to predict potential distribution of IMS. It is therefore recommended to use finer-scales and more realistic estimations of the suitable areas for IMS, with the aim of implementing appropriate surveillance and control measures.

\section{Management and control of mosquitoes}

In addition to nuisance, mosquitoes can pose a serious risk to public health (ECDC 2010). In Europe, autochthonous mosquito-borne outbreaks of dengue and chikungunya transmitted by recently established populations of IMS have occurred (Rezza et al. 2007, Gjenero-Margan et al. 2010, Gould et al. 2010, La Ruche et al. 2010, Sousa et al. 2012, Calba et al. 2017, Venturi et al. 2017). In addition, transmissions of malaria, West Nile disease or Usutu have also been associated with the presence of native mosquito species (Danis et al. 2011, Papa 2013, Sambri et al. 2013, Sieg et al. 2017). To control mosquitoes and the public health hazards they present, many European countries have established mosquito control programmes. Professional 'm osquito fighters' and m osquito experts in Europeform the core of the European Mosquito Control Association, EMCA, whose aim is to strengthen cooperation in technical and operational aspects of mosquito control (ECDC 2010).

Experience learnt in Europe (and other parts of the globe) confirms that efforts to control established populations of IMS or eradicate them are considered extremely difficult if not impossible (Paupy et al. 2009, Baldacchino et al. 2015). Therefore, timing of control actions is crucial for the success of managing introductions at an early stage. The policy of the Netherlands is to prevent the establishment of IMS in the country as long as possible. If an IMS foci is detected at an early stage in the invasion process, the policy is to enable control actions to prevent establishment. These control actions need to be proportional to the goal achieved in terms of sustainability, and the actions are analyzed for effectiveness before they are continued to be implemented. As discussed in Chapters 6, 7 and 8, after confirmed findings of IMS, evaluation of the proposed control measures is routinely conducted. Depending on different factors (species, location, vector status), management and control are applied at different scales. A combination of measures such as source reduction, larviciding (control of larvae), adulticiding (control of adults), can be applied in an integrated mosquito management (IMM) program in order to reduce the populations of mosquitoes in an area. For this task, permission for the use of the necessary biocides is often required. To date, biocides 
such as larvicides based on Bacillus thuringiensis israelensis (Bti) and Bacillus sphaericus (Bs), or adulticides based on deltamethrin, are still not fully authorized for use in mosquito control in the Netherlands.

In the Netherlands, except for the widely established population of Ae. japonicus in Lelystad, which was detected through indigenous mosquito surveillance, all other introductions detected since 2010 have been eradicated or are subjected to measures for it. Special attention is given to the control measures applied after the detection of Ae. albopictus through citizen nuisance notifications. In this case, breeding populations of this IMS were found in localized urban areas in several municipalities in the Netherlands (Veenendaal (2016), Weert (2016), and Aalten (2017), A. Ibañez-Justicia, unpublished data). Results of the intensive monitoring and control measures indicate that the populations in Veenendaal have been eradicated (unpublished data). In the locations Weert and Aalten, intensive monitoring has shown overwintering of the species (presence of the species after the winter months in breeding sites). Results of current surveillance and applied mosquito control in Weert and Aalten shows that the populations have been reduced and these incursions are under control (unpublished data). Considering the large number of detected introductions (see Chapter 6), the control implemented is considered successful and prevented future population buildup of IMS in the country. Therefore, the strategy, methodology and the implementation of control measures applied seem to be adequate for control in case of outbreaks of VBD. Furthermore, the reduction of Ae. japonicus populations after the IMM measures were applied at the allotments, has been shown them to be effective at these prolific sites (see Chapter 8). However, due to the number of other cryptic breeding sites in the area, eradication of $A e$. japonicus has not been considered realistic. If the control methods currently in use will be sufficient, the eradication of newly established populations of invasive mosquito species in the future will depend on the scale of the distribution area, the timing of the control and the season.

The use of synthetic chemicals such as pesticides in nature remains a taboo for ethical and ecological reasons (Anderberg 1998). For this reason, to reduce the use of biocides against larvae and adults novel biological alternatives, combined with effective source reduction, are suggested for IMM in case of VBD outbreaks. Examples of these alternatives are the use of predatory copepod species to control mosquito larvae (Marten et al. 1994, Dieng et al. 2002, Veronesi et al. 2015), the use of Wolbachia-infected mosquitoes to induce cytoplasmic incompatibility (Calvitti et al. 2012, Mains et al. 2016), the release of large numbers of 
sterilized mosquito males using the sterile insect technique (SIT) (Bellini et al. 2013b, Bellini et al. 2013a), or the use of amplified ultrasonic sound waves to kill mosquito larvae (Fredregill et al. 2015, Britch et al. 2016). Other tools such as entomopathogenic fungi, mass trapping and toxic sugar baits are not species specific, and hence less useful for control of invasive species.

\section{Concluding remarks}

The main pillars of this thesis are: 1) the development of surveillance strategies to obtain spatio-temporal data on mosquito species, 2) the study of the habitat suitability in the Netherlands for sustaining populations of endemic and exotic mosquito species using spatial distribution models, 3) the identification of the existing pathways for introduction and dispersal of invasive mosquito species, and 4) the analysis and evaluation of results of the implementation of risk-based and adaptive surveillance for IMS and mosquito control actions. My thesis has clearly shown that the geospatial occurrence data obtained through active surveillance is useful to study habitat suitability in the country for populations of $A n$. plumbeus, a potential malaria vector whose occurrence is highly correlated to environmental variables. In my opinion, without the countrywide active surveillance system as used for my thesis, the established populations of the IMS Ae. japonicus would probably have passed undetected by the general public, and would have been discovered accidently by amateur entomologists, or never discovered. Furthermore, the risk-based and adaptive surveillance of IMS described in my study revealed the annual introductions of Ae. albopictus since 2010 at used tire companies and Lucky bamboo greenhouses, sporadic findings of Ae. japonicus associated with used tires trade, the first aircraft associated import of Ae. aegypti in Europe, and the first associated Ae. japonicus import with Lucky bamboo plants elsewhere in the world. Control of these IMS after their detection has proven effective and avoided proliferation at these locations and their surroundings. However, if these IMS would be introduced via pathways outside the CMV risk-based surveillance (e.g. with private vehicles), the effectiveness of applied mosquito control measures could be compromised. In fact, introductions of IMS via passive transportation in ground vehicles have been highlighted in this thesis as one of the main dispersal pathways of Ae. albopictus within Europe, and these introductions can be expected in the future in the Netherlands as well. As mentioned in the previous section, populations of Ae. albopictus were found in urban areas outside the surveillance, and were notified to the NVWA-CMV by citizens using the NVWA website. 
Besides the active surveillance programme as currently conducted in the Netherlands, it is advised to supplement this programme with an improved passive surveillance system in order to provide additional scientific data and involve citizen collaboration on mosquito research. This will be of special interest when, besides reporting data on indigenous species, it could directly inform the NVWA-CMV of IMS findings at locations outside our surveillance coverage, using mobile phone applications (Palmer et al. 2017). Notifications of Ae. albopictus are expected to be reported, because the presence of this day biting species will be noticed (Lines 2007). Results of my research show that the current average climatic conditions can limit the overwintering of eggs of Ae. albopictus and their survival as adults in many inland areas of the country. However, due to the expected increase of the temperatures in the next decades as a result of climate change, these parts of the Netherlands may in future offer climatic conditions suitable for sustaining populations of this species. Due to the adaptability of Ae. albopictus to new environmental conditions, mosquito control actions applied to this IMS in order to eradicate early stage introductions are crucial for avoiding adaptation of the species to northern latitudes and preventing establishment. Even in the scenario that the species would establish in areas in the country, contrary to the tropics, mosquito adults will not survive the winter in the Netherlands. This fact can have important epidemiological consequences, because in case of VBD outbreaks, it might not allow viruses to survive the winter inside mosquitoes and to reappear in the spring. Thus, the work presented in my thesis provides essential insights into the presence and abundance of both indigenous and exotic mosquito species. This is important for identifying locations at risk of vector-borne disease transmission, and for designing targeted control of newly introduced exotic mosquito species in the Netherlands.

\section{Acknowledgements}

I thank Dr. Scott Gordon for improving the English on the first draft of this document. 



\section{References}


Adhami, J., and P. Reiter. 1998. Introduction and establishment of Aedes (Stegomyia) albopictus skuse (Diptera: Culicidae) in Albania. Journal of the American Mosquito Control Association 14: 340-343.

Ahmed J., M. Bouloy, O. Ergonul, A. Fooks, J. Paweska, V. Chevalier, C. Drosten, R. Moormann, N. Tordo, Z. Vatansever, P. Calistri, A. Estrada-Pena, A. Mirazimi, H. Unger, H. Yin, and U. Seitzer. 2009. International network for capacity building for the control of emerging viral vector-borne zoonotic diseases: ARBO-ZOONET. Eurosurveillance 14.

Akiner, M. M., B. Demirci, G. Babuadze, V. Robert, and F. Schaffner. 2016. Spread of the Invasive Mosquitoes Aedes aegypti and Aedes albopictus in the Black Sea Region Increases Risk of Chikungunya, Dengue, and Zika Outbreaks in Europe. PLoS Neglected Tropical Disesases 10: e0004664.

Anderberg, S. 1998. Industrial metabolism and the linkages between economics, ethics and the environment. Ecological Economics 24: 311-320.

Andriopoulos, P., A. Economopoulou, G. Spanakos, and G. Assimakopoulos. 2013. A local outbreak of autochthonous Plasmodium vivax malaria in Laconia, Greece--a reemerging infection in the southern borders of Europe? International Journal of Infectious Diseases 17: e125-128.

Aranda, C., R. Eritja, and D. Roiz. 2006. First record and establishment of the mosquito Aedes albopictus in Spain. Medical and Veterinary Entomology 20: 150-152.

Avia-GIS. 2012. Abundance modeling of mosquito and biting midge species in the Netherlands.

Avia-GIS. 2014. VecmapTM, A one-stop-shop for vector mapping, Zoersel, Belgium. http://www.avia-gis.com/vecmap. .

Baldacchino, F., B. Caputo, F. Chandre, A. Drago, A. della Torre, F. Montarsi, and A. Rizzoli. 2015. Control methods against invasive Aedes mosquitoes in Europe: a review. Pest Management Science 71: 1471-1485.

Baldwin, R. 2009. Use of Maximum Entropy Modeling in Wildlife Research. Entropy 11: 854.

Balenghien, T., M. Vazeille, M. Grandadam, F. Schaffner, H. Zeller, P. Reiter, P. Sabatier, F. Fouque, and D. J. Bicout. 2008. Vector competence of some French Culex and Aedes mosquitoes for West Nile virus. Vector Borne Zoonotic Diseases 8: 589-595.

Bara, J. J., A. T. Parker, and E. J. Muturi. 2016. Comparative Susceptibility of Ochlerotatus japonicus, Ochlerotatus triseriatus, Aedes albopictus, and Aedes aegypti (Diptera: Culicidae) to La Crosse Virus. Journal of Medical Entomology 53: 14151421.

Barceló, C., M. Bengoa, M. Monerris, R. Molina, S. Delacour-Estrella, and J. Lucientes. 2015. First record of Aedes albopictus (Skuse, 1894) (Diptera; Culicidae) from Ibiza (Balearic Islands; Spain). Journal of the European Mosquito Control Association 33: 14.

Baron, R. M., and D. A. Kenny. 1986. The moderator-mediator variable distinction in social psychological research: conceptual, strategic, and statistical considerations. Journal of Personality and Social Psychology 51: 1173-1182.

Bataille, A., A. A. Cunningham, V. Cedeno, M. Cruz, G. Eastwood, D. M. Fonseca, C. E. Causton, R. Azuero, J. Loayza, J. D. Martinez, and S. J. Goodman. 2009. Evidence for regular ongoing introductions of mosquito disease vectors into the Galapagos Islands. Proceedings of the Royal Society - biological sciences 276: 3769-3775. 
Becker, N., D. Petric, M. Zgomba, C. Boase, M. Madon,C. Dahl, and A. Kaiser. 2010. Mosquitoes and their control, Kluwer Academic/Plenum Publishers, Heidelberg, Dordrecht, London, New York.

Becker, N., H. Jost, U. Ziegler, M. Eiden, D. Hoper, P. Emmerich, E. Fichet-Calvet, D. U. Ehichioya, C. Czajka, M. Gabriel, B. Hoffmann, M. Beer, K. Tenner-Racz, P. Racz, S. Gunther, M. Wink, S. Bosch, A. Konrad, M. Pfeffer, M. H. Groschup, and J. Schmidt-Chanasit. 2012. Epizootic emergence of Usutu virus in wild and captive birds in Germany. PLoS One 7: e32604.

Becker, N., K. Huber, B. Pluskota, and A. Kaiser. 2011. Ochlerotatus japonicus japonicus - a neozoon in Germany and a revised list of the German mosquito fauna. European Mosquito Bulletin 9: 88-102.

Becker, N., M. Geier, C. Balczun, U. Bradersen, K. Huber, E. Kiel, A. Kruger, R. Luhken, C. Orendt, A. Plenge-Bonig, A. Rose, G. A. Schaub, and E. Tannich. 2013. Repeated introduction of Aedes albopictus into Germany, July to October 2012. Parasitology Research 112: 1787-1790.

Becker, N., S. Schon, A. M. Klein, I. Ferstl, A. Kizgin, E. Tannich, C. Kuhn, B. Pluskota, and A. Jost. 2017. First mass development of Aedes albopictus (Diptera: Culicidae)-its surveillance and control in Germany. Parasitology Research 116: 847858.

Beeuwkes, J., W. den Hartog, M. Dik, and E. Scholte. 2011. Surveillance and findings of exotic mosquitoes in used tires in The Netherlands: a methodological approach. Proceedings of the Netherlands Entomological Society Meeting 22.

Bellini, R., A. Medici, A. Puggioli, F. Balestrino, and M. Carrieri. 2013a. Pilot field trials with Aedes albopictus irradiated sterile males in Italian urban areas. Journal of Medical Entomology 50: 317-325.

Bellini, R., F. Balestrino, A. Medici, G. Gentile, R. Veronesi, and M. Carrieri. 2013b. Mating competitiveness of Aedes albopictus radio-sterilized males in large enclosures exposed to natural conditions. Journal of Medical Entomology 50: 94-102.

Benallal, K. E., A. Allal-Ikhlef, K. Benhamouda, F. Schaffner, and Z. Harrat. 2016. First report of Aedes (Stegomyia) albopictus (Diptera: Culicidae) in Oran, West of Algeria. Acta Tropica 164: 411-413.

Benedict, M.Q., R.S. Levine, W.A. Hawley, and L.P. Lounibos. 2007. Spread of the tiger: global risk of invasion by the mosquito Aedes albopictus. Vector Borne Zoonotic Diseases 7: 76-85.

Bengoa, M., S. Delacour-Estrella, C. Barceló, C. Paredes-Esquivel, M. Leza, J. Lucientes, R. Molina, and M. A. Miranda. 2016. First record of Aedes albopictus (Skuse, 1894) (Diptera; Culicidae) from Minorca (Balearic Islands, Spain). Journal of the European Mosquito Control Association 34: 5-9.

Bevins, S. N. 2007. Establishment and abundance of a recently introduced mosquito species Ochlerotatus japonicus (Diptera: Culicidae) in the Southern Appalachians, USA. Journal of Medical Entomology 44: 945-952.

Bezzhonova, O.V., I.V. Patraman, L.A. Ganushkina, O.I. Vyshemirskii, and V.P. Sergiev. 2014. The first finding of invasive species Aedes (Finlaya) koreicus (Edwards, 1917) in European Russia. Meditsinskaia parazitologiia i parazitarnye bolezni 16-19.

Bisanzio, D., M. Giacobini, L. Bertolotti, A. Mosca, L. Balbo, U. Kitron, and G. M. Vazquez-Prokopec. 2011. Spatio-temporal patterns of distribution of West Nile virus vectors in eastern Piedmont Region, Italy. Parasites \& Vectors 4: 230.

Boer, H., and E. R. Seydel. 1996. Protection motivation theory. In M. Conner, \& P. Norman (Eds.), Predicting Health Behaviour: Research and Practice with Social Cognition 
Models. Eds. Mark Conner, Paul Norman. Buckingham: Open University Press. (pp. 95-120).

Boyce, M.S., P.R. Vernier, S.E. Nielsen, and F.K. Schmiegelow. 2002. Evaluating resource selection functions. Ecological modelling 157: 281-300.

Bradshaw, W.E., and C.M. Holzapfel. 1991. Fitness and habitat segregation of British treehole mosquitoes. Ecological Entomology 16: 133-144.

Breiman, L. 2001. Random Forests. Machine Learning 45: 5-32.

Breiman, L., J.H. Friedman, R.A. Olshen, and C.J. Stone. 1984. Classification and regression trees. Wadsworth international group, Belmont, California, USA.

Briegel, H., A. Waltert, and A.R. Kuhn. 2001. Reproductive physiology of Aedes (Aedimorphus) vexans (Diptera: Culicidae) in relation to flight potential. Journal of Medical Entomology 38: 557-565.

Britch, S.C., H. Nyberg, R.L. Aldridge, T. Swan, and K.J. Linthicum. 2016. Acoustic control of mosquito larvae in artificial drinking water containers. Journal of the Americal Mosquito Control Association 32: 341-344.

Brown, J.E., B.R. Evans, W. Zheng, V. Obas, L. Barrera-Martinez, A. Egizi, H. Zhao, A. Caccone, and J.R. Powell. 2014. Human impacts have shaped historical and recent evolution in Aedes aegypti, the dengue and yellow fever mosquito. Evolution 68: 514525.

Brown, J.E., E.J. Scholte, M. Dik, W. Den Hartog, J. Beeuwkes, and J.R. Powell. 2011 a. Aedes aegypti mosquitoes imported into the Netherlands, 2010. Emerging Infectious Diseases 17: 2335-2337.

Brown, J.E., C.S. McBride, P. Johnson, S. Ritchie, C. Paupy, H. Bossin, J. Lutomiah, I. Fernandez-Salas, A. Ponlawat, A.J. Cornel, W.C.T. Black, N. GorrochoteguiEscalante, L. Urdaneta-Marquez, M. Sylla, M. Slotman, K.O. Murray, C. Walker, J.R. Powell. 2011b. Worldwide patterns of genetic differentiation imply multiple 'domestications' of Aedes aegypti, a major vector of human diseases. Proceedings of the Royal Society B: Biological Sciences 278: 2446-2454.

Bueno-Mari, R., and R. Jimenez-Peydro. 2012. Study of the malariogenic potential of Eastern Spain. Tropical biomedicine 29: 39-50.

Buhagiar, J. 2009. A second record of Aedes (Stegomyia) albopictus (Diptera: Culicidae) in Malta. European Mosquito Bulletin 27: 65-67.

Calba, C., M. Guerbois-Galla, F. Franke, C. Jeannin, M. Auzet-Caillaud, G. Grard, L. Pigaglio, A. Decoppet, J. Weicherding, M.C. Savaill, M. Munoz-Riviero, P. Chaud, B. Cadiou, L. Ramalli, P. Fournier, H. Noël, X. De Lamballerie, M.C. Paty, and I. Leparc-Goffart. 2017. Preliminary report of an autochthonous chikungunya outbreak in France, July to September 2017. Eurosurveillance 22: 17-00647.

Calvete, C., R. Estrada, M.A. Miranda, D. Borras, J.H. Calvo, and J. Lucientes. 2008. Modelling the distributions and spatial coincidence of bluetongue vectors Culicoides imicola and the Culicoides obsoletus group throughout the Iberian peninsula. Medical and Veterinary Entomololy 22: 124-134.

Calvitti, M., R. Moretti, A.R. Skidmore, and S.L. Dobson. 2012. Wolbachia strain wPip yields a pattern of cytoplasmic incompatibility enhancing a Wolbachia-based suppression strategy against the disease vector Aedes albopictus. Parasites \& Vectors 5: 254.

Calzolari, M., P. Bonilauri, R. Bellini, A. Albieri, F. Defilippo, G. Maioli, G. Galletti, A. Gelati, I. Barbieri, M. Tamba, D. Lelli, E. Carra, P. Cordioli, P. Angelini, and M. Dottori. 2010. Evidence of simultaneous circulation of West Nile and Usutu viruses in mosquitoes sampled in Emilia-Romagna region (Italy) in 2009. PLoS One 5: e14324. 
Caminade, C., J.M. Medlock, E. Ducheyne, K.M. McIntyre, S. Leach, M. Baylis, and A. P. Morse. 2012. Suitability of European climate for the Asian tiger mosquito Aedes albopictus: recent trends and future scenarios. Journal of The Royal Society Interface 9: 2708-2717.

Cancrini, G., P. Scaramozzino, S. Gabrielli, M. Di Paolo, L. Toma, and R. Romi. 2007. Aedes albopictus and Culex pipiens implicated as natural vectors of Dirofilaria repens in central Italy. Journal of Medical Entomology 44: 1064-1066.

Capelli, G., A. Drago, S. Martini, F. Montarsi, M. Soppelsa, N. Delai, S. Ravagnan, L. Mazzon, F. Schaffner, A. Mathis, M. Di Luca, R. Romi, and F. Russo. 2011. First report in Italy of the exotic mosquito species Aedes (Finlaya) koreicus, a potential vector of arboviruses and filariae. Parasites \& Vectors 4: 188.

CBS-Statistics-Netherlands. 2017. https://www.cbs.nl/en-gb.

CDC. 2015. Malaria transmission in the United States. https://www.cdc.gov/malaria/about/us_transmission.html.

Chefaoui, R.M., J. Hortal, and J.M. Lobo. 2005. Potential distribution modelling, niche characterization and conservation status assessment using GIS tools: a case study of Iberian Copris species. Biological Conservation 122: 327-338.

Cianci, D., N. Hartemink, and A. Ibañez-Justicia. 2015. Modelling the potential spatial distribution of mosquito species using three different techniques. International Journal of Health Geographics 14: 1.

Collantes, F., S. Delacour, P.M. Alarcon-Elbal, I. Ruiz-Arrondo, J.A. Delgado, A. Torrell-Sorio, M. Bengoa, R. Eritja, M.A. Miranda, R. Molina, and J. Lucientes. 2015. Review of ten-years presence of Aedes albopictus in Spain 2004-2014: known distribution and public health concerns. Parasites \& Vectors 8: 655.

Connelly, C.R., and D.B. Carlson. 2009. Florida Mosquito Control: The state of the mission as defined by mosquito controllers, regulators, and environmental managers. University of Florida, Institute of Food and Agricultural Sciences, Florida Medical Entomology Laboratory, Florida USA.

Cronbach, L.J. 1951. Coefficient alpha and the internal structure of tests. Psychometrika 16: 297-334.

Cunze, S., L.K. Koch, J. Kochmann, and S. Klimpel. 2016a. Aedes albopictus and Aedes japonicus - two invasive mosquito species with different temperature niches in Europe. Parasites \& Vectors 9: 573.

Cunze, S., J. Kochmann, L.K. Koch, and S. Klimpel. 2016b. Aedes albopictus and its environmental limits in Europe. PLoS One 11: e0162116.

Cutler, D.R., T.C. Edwards, Jr., K.H. Beard, A. Cutler, K.T. Hess, J. Gibson, and J.J. Lawler. 2007. Random forests for classification in ecology. Ecology 88: 2783-2792.

Dalla Pozza, G., and G. Majori. 1992. First record of Aedes albopictus establishment in Italy. Journal of the American Mosquito Control Association 8: 318-320.

Damiens, D., A. Ayrinhac, W. Van Bortel, V. Versteirt, W. Dekoninck, and T. Hance. 2014. Invasive process and repeated cross-sectional surveys of the mosquito Aedes japonicus japonicus establishment in Belgium. PLoS ONE 9(4):e89358.

Danis, K., A. Baka, A. Lenglet, W. Van Bortel, I. Terzaki, M. Tseroni, M. Detsis, E. Papanikolaou, A. Balaska, S. Gewehr, G. Dougas, T. Sideroglou, A. Economopoulou, N. Vakalis, S. Tsiodras, S. Bonovas, and J. Kremastinou. 2011. Autochthonous Plasmodium vivax malaria in Greece, 2011. Eurosurveillance 16: 19993

de Azara T.M., C.M. Degener, R.A. Roque, J.J. Ohly, M. Geier, A.E. Eiras. 2013. The impact of $\mathrm{CO} 2$ on collection of Aedes aegypti (Linnaeus) and Culex quinquefasciatus Say by BG-Sentinel traps in Manaus, Brazil. Memórias do Instituto Oswaldo Cruz 108: 229-232. 
de Lamballerie, X., E. Leroy, R.N. Charrel, K. Ttsetsarkin, S. Higgs, E.A. Gould. 2008. Chikungunya virus adapts to tiger mosquito via evolutionary convergence: a sign of things to come? Virology Journal 5: 33.

Degener, C.M., T.M. Azara, R.A. Roque, C.T. Codeco, A.A. Nobre, J.J. Ohly, M. Geier, A.E. Eiras. 2014. Temporal abundance of Aedes aegypti in Manaus, Brazil, measured by two trap types for adult mosquitoes. Memórias do Instituto Oswaldo Cruz 109: 1030-1040.

Dekoninck, W., F. Hendrickx, W. Van Bortel, V. Versteirt, M. Coosemans, D. Damiens, T. Hance, E.M. De Clercq, G. Hendrickx, F. Schaffner, and P. Grootaert. 2011. Human-induced expanded distribution of Anopheles plumbeus, experimental vector of West Nile virus and a potential vector of human malaria in Belgium. Journal of Medical Entomology 48: 924-928.

Delacour, S., J.F. Barandika, A.L. García-Pérez, F. Collantes, I. Ruiz-Arrondo, P.M. Alarcón-Elbal, M. Bengoa, J.A. Delgado, R.A. Juste, R. Molina, and J. Lucientes. 2015. Detección temprana del mosquito tigre, Aedes albopictus (Skuse, 1894), en el País Vasco (España). Anales de Biología 37: 25-30.

Delatte, H., C. Paupy, J.S. Dehecq, J. Thiria, A.B. Failloux, and D. Fontenille. 2008. Aedes albopictus, vector of chikungunya and dengue viruses in Reunion island: biology and control. Parasite 15: 3-13.

Demeulemeester, J., I. Deblauwe, J. De Witte, F. Jansen, A. Hendy, and M. Madder. 2014. First interception of Aedes (Stegomyia) albopictus in Lucky bamboo shipments in Belgium. Journal of the European Mosquito Control Association 32: 14-16.

Dennett, J.A., N.Y. Vessey, and R.E. Parsons. 2004. A comparison of seven traps used for collection of Aedes albopictus and Aedes aegypti originating from a large tire repository in Harris County (Houston), Texas. Journal of the American Mosquito Control Association 20: 342-349.

Derraik, J.G. 2004. Exotic mosquitoes in New Zealand: a review of species intercepted, their pathways and ports of entry. Australian and New Zealand Journal of Public Health 28: 433-444.

Di Luca, M., L. Toma, F. Severini, D. Boccolini, S. D'Avola, D. Todaro, A. Stancanelli, F. Antoci, F. La Russa, S. Casano, S.D. Sotera, E. Carraffa, V. Versteirt, F. Schaffner, R. Romi, and A. Torina. 2017. First record of the invasive mosquito species Aedes (Stegomyia) albopictus (Diptera: Culicidae) on the southernmost Mediterranean islands of Italy and Europe. Parasites \& Vectors 10: 543.

Dieng, H., M. Boots, N. Tuno, Y. Tsuda, and M. Takagi. 2002. A laboratory and field evaluation of Macrocyclops distinctus, Megacyclops viridis and Mesocyclops pehpeiensis as control agents of the dengue vector Aedes albopictus in a peridomestic area in Nagasaki, Japan. Medical and Veterinary Entomology 16: 285-291.

Direction Générale Opérationnelle Agriculture Ressources naturelles et Environnement. 2017. Le moustique japonais en Wallonie. http://biodiversite.wallonie.be/fr/le-moustique-japonais.html?IDC=6000

ECDC \& EFSA. 2018. Surveillance and disease data for disease vectors. https://ecdc.europa.eu/en/disease-vectors/surveillance-and-disease-data.

ECDC. 2009. Development of Aedes albopictus risk maps. https://ecdc.europa.eu/sites/portal/files/media/en/publications/Publications/0905_TER_ Development_of_Aedes_Albopictus_Risk_Maps.pdf

ECDC. 2010. Mosquito control methods. https://ecdc.europa.eu/en/news-events/mosquitocontrol-methods-europe. 
ECDC. 2012. Guidelines for the surveillance of invasive mosquitoes in Europe. https://ecdc.europa.eu/sites/portal/files/media/en/publications/Publications/TERMosquito-surveillance-guidelines.pdf.

ECDC. 2013. Environmental risk mapping: Aedes albopictus in Europe. http://www.ecdc.europa.eu/en/publications/Publications/climate-change-environmentalrisk-mapping-aedes.pdf.

ECDC. 2014a. Guidelines for the surveillance of native mosquitoes in Europe. https://ecdc.europa.eu/sites/portal/files/media/en/publications/Publications/surveillanceof $\% 20$ native-mosquitoes $\% 20$-guidelines.pdf.

ECDC. 2014b. Aedes triseriatus - Factsheet for experts. https://ecdc.europa.eu/en/diseasevectors/facts/mosquito-factsheets/aedes-triseriatus

ECDC. 2014c. Aedes atropalpus - Factsheet for experts. https://ecdc.europa.eu/en/diseasevectors/facts/mosquito-factsheets/aedes-atropalpus.

ECDC. 2016. Aedes albopictus - Factsheet for experts. https://ecdc.europa.eu/en/diseasevectors/facts/mosquito-factsheets/aedes-albopictus.

ECDC. 2017. Mosquito factsheets. https://ecdc.europa.eu/en/disease-vectors/facts/mosquitofactsheets.

ECDC. 2018a. Vector-borne diseases. https:/ecdc.europa.eu/en/climate-change/climatechange-europe/vector-borne-diseases.

ECDC. 2018b. New settlements of Aedes aegypti raising concerns for continental EU. https://ecdc.europa.eu/en/news-events/new-settlements-aedes-aegypti-raising-concernscontinental-eu.

ECDC. 2018c. Aedes albopictus - current known distribution: January 2018. https://ecdc.europa.eu/en/publications-data/aedes-albopictus-current-knowndistribution-january-2018.

Egizi, A., J. Kiser, C. Abadam, and D.M. Fonseca. 2016. The hitchhiker's guide to becoming invasive: Exotic mosquitoes spread across a US state by human transport not autonomous flight. Molecular Ecology 25(13):3033-47

Eisen, L., and C.G. Moore. 2013. Aedes (Stegomyia) aegypti in the continental United States: a vector at the cool margin of its geographic range. Journal of Medical Entomology 50: 467-478.

Elith, J., and J.R. Leathwick. 2009. Species distribution models: Ecological explanation and prediction across space and time. Annual Review of Ecology, Evolution, and Systematics 40: 677-697.

Elith, J., C.H. Graham, R.P. Anderson, M. Dudík, S. Ferrier, A. Guisan, R.J. Hijmans, F. Huettmann, J.R. Leathwick, A. Lehmann, J. Li, L.G. Lohmann, B.A. Loiselle, G. Manion, C. Moritz, M. Nakamura, Y. Nakazawa, J. McC. M. Overton, A. Townsend Peterson, S.J. Phillips, K. Richardson, R. Scachetti-Pereira, R.E. Schapire, J. Soberón, S. Williams, M.S. Wisz, and N.E. Zimmermann. 2006. Novel $\mathrm{m}$ ethods im prove prediction of species' distributions from occurrence data. Ecography 29: $129-151$.

EMCA. 2017. Abstract book VIIIth Conference 12-16 March 2017 Bečići Montenegro: Mosquito Control in a Changing Environment. p.128.

Enserink, M. 2010. Infectious Diseases. Yellow fever mosquito shows up in Northern Europe. Science 329: 736.

Eritja, R., J.R.B. Palmer, D. Roiz, I. Sanpera-Calbet, and F. Bartumeus. 2017. Direct evidence of adult Aedes albopictus dispersal by car. Science Reports 7: 14399.

European Environment Agency. www.eea.europa.eu. 
Ewing, D. A., C.A. Cobbold, B.V. Purse, M.A. Nunn, and S.M. White. 2016. Modelling the effect of temperature on the seasonal population dynamics of temperate mosquitoes. Journal of Theoretical Biology 400: 65-79.

Farajollahi, A., D.M. Fonseca, L.D. Kramer, and A. Marm Kilpatrick. 2011. "Bird biting" mosquitoes and human disease: a review of the role of Culex pipiens complex mosquitoes in epidemiology. Infection, Genetics and Evolution 11: 1577-1585.

Fielding, A.H., and J.F. Bell. 1997. A review of methods for the assessment of prediction errors in conservation presence/absence models. Environmental conservation 24: 38-49.

Fischer, D., S.M. Thomas, F. Niemitz, B. Reineking, and C. Beierkuhnlein. 2011. Projection of climatic suitability for Aedes albopictus Skuse (Culicidae) in Europe under climate change conditions. Global and Planetary Change 78: 54-64.

Fischer, D., S.M. Thomas, M. Neteler, N.B. Tjaden, and C. Beierkuhnlein. 2014. Climatic suitability of Aedes albopictus in Europe referring to climate change projections: comparison of mechanistic and correlative niche modelling approaches. Eurosurveillance 19: 20696

Fischer, E.A., G.J. Boender, G. Nodelijk, A.A. de Koeijer, and H.J. van Roermund. 2013. The transmission potential of Rift Valley fever virus among livestock in the Netherlands: a modelling study. Veterinary Research 44: 58.

Flacio, E., L. Engeler, M. Tonolla, and P. Muller. 2016. Spread and establishment of Aedes albopictus in southern Switzerland between 2003 and 2014: an analysis of oviposition data and weather conditions. Parasites \& Vectors 9: 304.

Floyd, D.L., S. Prentice-Dunn, and R.W. Rogers. 2000. A meta-analysis of research on protection motivation theory. Journal of applied social psychology 30: 407-429.

Fontenille, D., M. Diallo, M. Mondo, M. Ndiaye, and J. Thonnon. 1997. First evidence of natural vertical transmission of yellow fever virus in Aedes aegypti, its epidemic vector. Transactions of the Royal Society of Tropical Medicine and Hygiene 91: 533-535.

Fredregill, C.L., G.C. Motl, J.A. Dennett, R. Bueno, Jr., and M. Debboun. 2015. Efficacy of two larvasonic units against Culex larvae and effects on common aquatic nontarget organisms in Harris County, Texas. Journal of the American Mosquito Control Association 31: 366-370.

Gaspar, J.P., T. McKay, and M.J. Huss. 2012. First report of Aedes japonicus in natural and artificial habitats in Northeastern Arkansas. Journal of the American Mosquito Control Association 28: 38-42.

Generalitat de Catalunya. 2008. Caracterització de la població del mosquit tigre asiàtic (Aedes albopictus) a Catalunya 2008. Departament de Medi Ambient i Habitatge, Direcció General del Medi Natural. http://premsa.gencat.cat/pres_fsvp/docs/2010/10/29/13/29/3a2c0857-53da-4e38-a060102e63e56544.pdf.

Gerhardt, R.R., K.L. Gottfried, C.S. Apperson, B.S. Davis, P.C. Erwin, A.B. Smith, N. A. Panella, E.E. Powell, and R.S. Nasci. 2001. First isolation of La Crosse virus from naturally infected Aedes albopictus. Emerging Infectious Diseases 7: 807-811.

Gezairy, H.A. 2003. Travel epidemiology: WHO perspective. International Journal of Antimicrobial Agents 21: 86-88.

Giacomini, T., J. Mouchet, P. Mathieu, and J.C. Petithory. 1995. Study on six cases of malaria contracted near Roissy-Charles de Gaulle in 1994. Preventive measures necessary in airports. Bulletin de l'Académie Nationale de Médecine (France) 179: 335-353.

Gibson, L., B. Wilson, D. Cahill, and J. Hill. 2004. Spatial prediction of rufous bristlebird habitat in a coastal heathland: a GIS-based approach. Journal of applied ecology 41: 213-223. 
Gjenero-Margan, I., B. Aleraj, D. Krajcar, V. Lesnikar, A. Klobucar, I. Pem-Novosel, S. Kurecic-Filipovic, S. Komparak, R. Martic, S. Duricic, L. Betica-Radic, J. Okmadzic, T. Vilibic-Cavlek, A. Babic-Erceg, B. Turkovic, T. Avsic-Zupanc, I. Radic, M. Ljubic, K. Sarac, N. Benic, and G. Mlinaric-Galinovic. 2010. Autochthonous dengue fever in Croatia, August-September 2010. Eurosurveillance 16: 19805

Gloria-Soria, A., D. Ayala, A. Bheecarry, O. Calderon-Arguedas, D.D. Chadee, M. Chiappero, M. Coetzee, K.B. Elahee, I. Fernandez-Salas, H.A. Kamal, B. Kamgang, E.I. Khater, L.D. Kramer, V. Kramer, A. Lopez-Solis, J. Lutomiah, A. Martins, Jr., M.V. Micieli, C. Paupy, A. Ponlawat, N. Rahola, S.B. Rasheed, J.B. Richardson, A.A. Saleh, R.M. Sanchez-Casas, G. Seixas, C.A. Sousa, W.J. Tabachnick, A. Troyo, J.R. Powell. 2016. Global genetic diversity of Aedes aegypti. Molecular Ecology 25: 5377-5395.

Gould, E.A., P. Gallian, X. De Lamballerie, and R.N. Charrel. 2010. First cases of autochthonous dengue fever and chikungunya fever in France: from bad dream to reality! Clinical Microbiology and Infection 16: 1702-1704.

Gratz, N.G. 1999. Emerging and resurging vector-borne diseases. Annual Review of Entomology 44: 51-75.

Gratz, N.G. 2004. Critical review of the vector status of Aedes albopictus. Medical Veterinary Entomology 18: 215-227.

Gratz, N.G., R. Steffen, and W. Cocksedge. 2000. Why aircraft disinsection? Bulletin World Health Organisation 78: 995-1004.

Guerenstein, P.G., and J.G. Hildebrand. 2008. Roles and effects of environmental carbon dioxide in insect life. Annual Review of Entomology 53: 161-178.

Guo, Z., S.D. Wang, M.M. Cheng, and Y. Shu. 2012. Assess the effect of different degrees of urbanization on land surface temperature using remote sensing images. Procedia Environmental Sciences 13: 935-942.

Gura, T. 2013. Citizen science: amateur experts. Nature 496: 259-261.

Gutsevich, A.V. and A.M. Dubitskyi. 1987. New species of mosquitoes in the fauna of the USSR. Mosquito Systematics 19: 1-92.

Harris, M.C., E.J. Dotseth, B.T. Jackson, S.D. Zink, P.E. Marek, L.D. Kramer, S.L. Paulson, and D.M. Hawley. 2015. La Crosse Virus in Aedes japonicus japonicus mosquitoes in the Appalachian Region, United States. Emerging Infectious Diseases 21: 646-649.

Hartberg, W.K. 1971. Observations on the mating behaviour of Aedes aegypti in nature. Bulletin World Health Organisation 45: 847-850.

Hartemink, N., S.O. Vanwambeke, H. Heesterbeek, D. Rogers, D. Morley, B. Pesson, C. Davies, S. Mahamdallie, and P. Ready. 2011. Integrated mapping of establishment risk for emerging vector-borne infections: a case study of canine leishmaniasis in southwest France. PLoS One 6: e20817.

Hartemink, N.A., B.V. Purse, R. Meiswinkel, H.E. Brown, A. de Koeijer, A.R. Elbers, G.J. Boender, D.J. Rogers, and J.A. Heesterbeek. 2009. Mapping the basic reproduction number $(\mathrm{R}(0))$ for vector-borne diseases: a case study on bluetongue virus. Epidemics 1: 153-161.

Hawley, W.A. 1988. The biology of Aedes albopictus. Journal of the American Mosquito Control Association Suppl 1: 1-39.

Hawley, W.A., P. Reiter, R.S. Copeland, C.B. Pumpuni, and G.B. Craig, Jr. 1987. Aedes albopictus in North America: probable introduction in used tires from northern Asia. Science 236: 1114-1116. 
Hesson, J.C., O. Ostman, M. Schafer, and J.O. Lundstrom. 2011. Geographic distribution and relative abundance of the sibling vector species Culex torrentium and Culex pipiens in Sweden. Vector Borne Zoonotic Dis 11: 1383-1389.

Heym, E.C., J. Schroder, H. Kampen, and D. Walther. 2017. The nuisance mosquito Anopheles plumbeus (Stephens, 1828) in Germany - A questionnaire survey may help support surveillance and control. Frontiers Public Health 5: 278.

Higa, Y. 2011. Dengue Vectors and their Spatial Distribution. Tropical Medicine and Health 39: 17-27.

Hodge, V.J., and J. Austin. 2004. A survey of outlier detection methodologies. Artificial Intelligence Review 22: 85-126.

Hoel, D.F., D.L. Kline, and S.A. Allan. 2009. Evaluation of six mosquito traps for collection of Aedes albopictus and associated mosquito species in a suburban setting in north central Florida. Journal of the American Mosquito Control Association 25: 47-57.

Hofhuis, A., J. Reimerink, C. Reusken, E.J. Scholte, A. Boer, W. Takken, and M. Koopmans. 2009. The hidden passenger of lucky bamboo: do imported Aedes albopictus mosquitoes cause dengue virus transmission in the Netherlands? Vector Borne Zoonotic Dis 9: 217-220.

Honorio, N.A., C. Silva Wda, P.J. Leite, J.M. Goncalves, L.P. Lounibos, and R. Lourenco-de-Oliveira. 2003. Dispersal of Aedes aegypti and Aedes albopictus (Diptera: Culicidae) in an urban endemic dengue area in the State of Rio de Janeiro, Brazil. Mem Inst Oswaldo Cruz 98: 191-198.

Hubalek, Z., I. Rudolf, T. Bakonyi, K. Kazdova, J. Halouzka, O. Sebesta, S. Sikutova, Z. Juricova, and N. Nowotny. 2010. Mosquito (Diptera: Culicidae) surveillance for arboviruses in an area endemic for West Nile (Lineage Rabensburg) and Tahyna viruses in Central Europe. Journal of Medical Entomology 47: 466-472.

Hughes, J.H. 1961. Mosquito interceptions and related problems in aerial traffic arriving in the United States. Mosquito News: 093-100.

Ibañez-Justicia, A., A. Gloria-Soria, W. den Hartog, M. Dik, F. Jacobs, and A. Stroo. 2017. The first detected airline introductions of yellow fever mosquitoes (Aedes aegypti) to Europe, at Schiphol International airport, the Netherlands. Parasites \& Vectors 10: 603.

Ibañez-Justicia, A., A. Stroo, M. Dik, J. Beeuwkes, and E. J. Scholte. 2015. National Mosquito (Diptera: Culicidae) Survey in The Netherlands 2010-2013. Journal of Medical Entomology 52: 185-198.

Ibañez-Justicia, A., H. Kampen, M. Braks, F. Schaffner, M. Steeghs, D. Werner, D. Zielke, W. den Hartog, M. Brooks, M. Dik, B. van de Vossenberg, and E.J. Scholte. 2014. First report of Aedes japonicus japonicus (Theobald, 1901) (Diptera, Culicidae) population in The Netherlands. Journal of the European Mosquito Control Association 32: 9-13.

Ibañez-Justicia, A., S. Teekema, W. den Hartog, F. Jacobs, M. Dik, and A. Stroo. 2018 a. The Effectiveness of Asian Bush Mosquito (Aedes japonicus japonicus) Control Actions in Colonised Peri-urban Areas in the Netherlands. Journal of Medical Entomology 55: 673-680.

Ibañez-Justicia, A., C.J.M. Koenraadt, A. Stroo, R. van Lammeren, and W. Takken. 2018b. Risk-based and adaptive surveillance at Lucky bamboo and used tire importers to prevent the establishment of invasive mosquitoes in the Netherlands. In this thesis.

Janz, N.K., and M.H. Becker. 1984. The Health Belief Model: a decade later. Health education quarterly 11: 1-47.

Jordan, R.C., A.E. Sorensen, and S. Ladeau. 2017. Citizen Science as a tool for mosquito control. Journal of the American Mosquito Control Association 33: 241-245. 
Jupille, H., G. Seixas, L. Mousson, C.A. Sousa, and A.B. Failloux. 2016. Zika Virus, a New Threat for Europe? PLoS Neglected Tropical Diseases 10: e0004901.

Justice, C., J. Townshend, E. Vermote, R. Sohlberg, J. Descloitres, D. Roy, D. Hall, V. Salomonson, G. Riggs, A. Huerte, K. Didan, T. Miura, W. Zhengming, A. Strahler, C. Schaaf, R. Myneni, S. Running, J. Glassy, R. Nemani, N.E. Saleous, and R. Wolfe. 2000. Preliminary land surface products from the NASA Moderate Resolution Imaging Spectroradiometer (MODIS), pp. 1157-1162 vol.1153. In, IGARSS 2000. IEEE 2000 International Geoscience and Remote Sensing Symposium. Taking the Pulse of the Planet: The Role of Remote Sensing in Managing the Environment. Proceedings (Cat. No.00CH37120).

K alan, K ., V. Buzan, and V. Ivović. 2014.Distribution of two invasive mosquito species in Slovenia in 2013. Parasites \& Vectors 7: P9.

Kalan, K., V. Ivovic, P. Glasnovic, and E. Buzan. 2017. Presence and potential distribution of Aedes albopictus and Aedes japonicus japonicus (Diptera: Culicidae) in Slovenia. Journal of Medical Entomology 54: 1510-1518.

Kamgang, B., E. Nchoutpouen, F. Simard, and C. Paupy. 2012. Notes on the bloodfeeding behavior of Aedes albopictus (Diptera: Culicidae) in Cameroon. Parasites \& Vectors 5: 57.

Kampen, H., and D. Werner. 2014. Out of the bush: the Asian bush mosquito Aedes japonicus japonicus (Theobald, 1901) (Diptera, Culicidae) becomes invasive. Parasites \& Vectors 7: 59.

Kampen, H., C. Kuhlisch, A. Fröhlich, D.E. Scheuch, and D. Walther. 2016. Occurrence and spread of the invasive asian bush mosquito Aedes japonicus japonicus (Diptera: Culicidae) in west and north Germany since detection in 2012 and 2013, respectively. PLoS ONE 11.

Kampen, H., D. Zielke, and D. Werner. 2012. A new focus of Aedes japonicus japonicus (Theobald, 1901) (Diptera, Culicidae) distribution in Western Germany: rapid spread or a further introduction event? Parasites \& Vectors 5: 284.

Kampen, H., J.M. Medlock, A.G. Vaux, C.J. Koenraadt, A.J. van Vliet, F. Bartumeus, A. Oltra, C.A. Sousa, S. Chouin, and D. Werner. 2015. Approaches to passive mosquito surveillance in the EU. Parasites \& Vectors 8: 9.

Kampen, H., M. Kronefeld, D. Zielke, and D. Werner. 2013. Further specimens of the Asian tiger mosquito Aedes albopictus (Diptera, Culicidae) trapped in southwest Germany. Parasitology Research 112: 905-907.

Kampen, H., S. Jansen, J. Schmidt-Chanasit, and D. Walther. 2016. Indoor development of Aedes aegypti in Germany, 2016. Eurosurveillance 21: 30407

Kaufman, M.G., and D.M. Fonseca. 2014. Invasion biology of Aedes japonicus japonicus (Diptera: Culicidae). Annual Review Entomology 59: 31-49.

Kilpatrick, A.M., and S.E. Randolph. 2012. Drivers, dynamics, and control of emerging vector-borne zoonotic diseases. Lancet 380: 1946-1955.

Kilpatrick, A.M., P. Daszak, S.J. Goodman, H. Rogg, L.D. Kramer, V. Cedeno, and A. A. Cunningham. 2006. Predicting pathogen introduction: West Nile virus spread to Galaipagos. Conservation Biology 20: 1224-1231.

Klobucar, A., I. Lipovac, N. Zagar, S. Mitrovic-Hamzic, V. Tesic, T. Vilibic-Cavlek, and E. Merdic. 2018. First record and spreading of the invasive mosquito Aedes japonicus japonicus (Theobald, 1901) in Croatia. Medical Veterinary Entomology doi: $10.1111 /$ mve. 12337

KNMI. 2014. KNMI'14: Clim ate Change scenarios for the 21st Century- A Netherlands perspective. http://www.klimaatscenarios.nl/brochures/images/KNMI_WR_201401_version26May2014.pdf. 
KNMI. 2018. Regional differences in the extreme rainfall climatology in the Netherlands. https://www.knmi.nl/kennis-en-datacentrum/achtergrond/regional-differences-in-theextreme-rainfall-climatology-in-the-netherlands.

Knottnerus, O.S. 2002. Malaria Around the North Sea: A Survey, in: Gerold Wefer, Wolfgang H. Berger, Karl-Ernst Behre, Eynstein Jansen (ed.), Climatic Development and History of the North Atlantic Realm: Hanse Conference Report. , Berlin, Germany.

Knudsen, A.B., R. Romi, and G. Majori. 1996. Occurrence and spread in Italy of Aedes albopictus, with implications for its introduction into other parts of Europe. Journal American Mosquito Control Association 12: 177-183.

Kobayashi, M., N. Nihei, and T. Kurihara. 2002. Analysis of northern distribution of Aedes albopictus (Diptera: Culicidae) in Japan by geographical information system. Journal Medical Entomology 39: 4-11.

Koch, L.K., S. Cunze, A. Werblow, J. Kochmann, D.D. Dorge, H. Mehlhorn, and S. Klimpel. 2016. Modeling the habitat suitability for the arbovirus vector Aedes albopictus (Diptera: Culicidae) in Germany. Parasitology Research 115: 957-964.

Kraemer, M.U., M.E. Sinka, K.A. Duda, A.Q. Mylne, F.M. Shearer, C.M. Barker, C.G. Moore, R.G. Carvalho, G.E. Coelho, W. Van Bortel, G. Hendrickx, F. Schaffner, I. R. Elyazar, H.J. Teng, O.J. Brady, J.P. Messina, D.M. Pigott, T.W. Scott, D.L. Smith, G.R. Wint, N. Golding, and S. I. Hay. 2015a. The global distribution of the arbovirus vectors Aedes aegypti and Ae. albopictus. Elife 4: e08347.

Kraemer, M.U., M.E. Sinka, K.A. Duda, A. Mylne, F.M. Shearer, O.J. Brady, J.P. Messina, C.M. Barker, C.G. Moore, R.G. Carvalho, G.E. Coelho, W. Van Bortel, G. Hendrickx, F. Schaffner, G.R. Wint, I.R. Elyazar, H.J. Teng, and S.I. Hay. 2015b. The global compendium of Aedes aegypti and Ae. albopictus occurrence. Science Data 2: 150035.

Krebs, C.J. 1989. Ecological methodology. Harper and Row Publishers, New York, NY.

Kruger, A., A. Rech, X.Z. Su, and E. Tannich. 2001. Two cases of autochthonous Plasmodium falciparum malaria in Germany with evidence for local transmission by indigenous Anopheles plumbeus. Tropical Medicine \& International Health 6: 983-985.

Kurucz, K., V. Kiss, B. Zana, V. Schmieder, A. Kepner, F. Jakab, and G. Kemenesi. 2016. Emergence of Aedes koreicus (Diptera: Culicidae) in an urban area, Hungary, 2016. Parasitology Research 115: 4687-4689.

La Ruche, G., Y. Souares, A. Armengaud, F. Peloux-Petiot, P. Delaunay, P. Despres, A. Lenglet, F. Jourdain, I. Leparc-Goffart, F. Charlet, L. Ollier, K. Mantey, T. Mollet, J.P. Fournier, R. Torrents, K. Leitmeyer, P. Hilairet, H. Zeller, W. Van Bortel, D. Dejour-Salamanca, M. Grandadam, and M. Gastellu-Etchegorry. 2010. First two autochthonous dengue virus infections in metropolitan France, September 2010. Eurosurveillance 15: 19676.

Liew, C., and C.F. Curtis. 2004. Horizontal and vertical dispersal of dengue vector mosquitoes, Aedes aegypti and Aedes albopictus, in Singapore. Medical Veterinary Entomology 18: 351-360.

Lima, A., D.D. Lovin, P.V. Hickner, and D.W. Severson. 2016. Evidence for an overwintering population of Aedes aegypti in Capitol Hill Neighborhood, Washington, DC. The american journal of tropical medicine and hygiene 94: 231-235.

Lines, J. 2007. Chikungunya in Italy. BMJ 335: 576.

Linthicum, K.J., V.L. Kramer, M.B. Madon, K. Fujioka, and Team Surveillance \& Control. 2003. Introduction and potential establishment of Aedes albopictus in California in 2001. Journal of American mosquito control association 19: 301-308.

Lobo, J.M., A. Jiménez-Valverde, and J. Hortal. 2010. The uncertain nature of absences and their importance in species distribution modelling. Ecography (Cop.) 33: 103-114. 
Lounibos, L.P. 2002. Invasions by insect vectors of human disease. Annual Review Entomology 47: 233-266.

Lundstrom, J. O. 1999. Mosquito-borne viruses in western Europe: a review. Journal Vector Ecology 24: 1-39.

Madon, M.B., J.E. Hazelrigg, M.W. Shaw, S. Kluh, and M.S. Mulla. 2003. Has Aedes albopictus established in California? Journal of American mosquito control association 19: 297-300.

Madon, M.B., M.S. Mulla, M.W. Shaw, S. Kluh, and J.E. Hazelrigg. 2002. Introduction of Aedes albopictus (Skuse) in southern California and potential for its establishment. Journal of Vector Ecology 27: 149-154.

Mains, J.W., C.L. Brelsfoard, R.I. Rose, and S.L. Dobson. 2016. Female adult Aedes albopictus suppression by Wolbachia-infected male mosquitoes. Science Reports 6:33846.

Mangili, A., and M.A. Gendreau. 2005. Transmission of infectious diseases during commercial air travel. The Lancet 365: 989-996.

Marcantonio, M., M. Metz, F. Baldacchino, D. Arnoldi, F. Montarsi, G. Capelli, S. Carlin, M. Neteler, and A. Rizzoli. 2016. First assessment of potential distribution and dispersal capacity of the emerging invasive mosquito Aedes koreicus in Northeast Italy. Parasites \& Vectors 9: 63.

Marchette, N.J., R. Garcia, and A. Rudnick. 1969. Isolation of Zika virus from Aedes aegypti mosquitoes in Malaysia. The American Journal of Tropical Medicine and Hygiene 18: 411-415.

Marshall, J. F. 1938. The British mosquitoes. Brit. Mus. (Nat. Hist.), London. 341.

Marten, G.G., G. Borjas, M. Cush, E. Fernandez, and J.W. Reid. 1994. Control of larval Aedes aegypti (Diptera: Culicidae) by cyclopoid copepods in peridomestic breeding containers. Journal of Medical Entomology 31: 36-44.

McCullagh, P., and J.A. Nelder. 1989. Generalized linear models. CRC press vol. 37.

McKenzie, K.E., and S.D. Bedard. 2004. A comparison of the efficacy of several models of Mosquito Magnet ${ }^{\circledR}$ traps baited with Lurex3 versus commercially available traps supplied with octenol on the collection of mosquitoes in Oahu, Hawaii, Primarily Aedes albopictus, September 2004. http://www.mosquitomagnet.com/resource/documents/ MM_Research/HawaiiTestingSummaryrev2.pdf.

Medlock, J.M., A.G.C. Vaux, B. Cull, F. Schaffner, E. Gillingham, V. Pfluger, and S. Leach. 2017. Detection of the invasive mosquito species Aedes albopictus in southern England. The Lancet Infectious Diseases 17: 140.

Medlock, J.M., and A.G. Vaux. 2015. Impacts of the creation, expansion and management of English wetlands on mosquito presence and abundance - developing strategies for future disease mitigation. Parasites \& Vectors 8: 142.

Medlock, J.M., D. Avenell, I. Barrass, and S. Leach. 2006. Analysis of the potential for survival and seasonal activity of Aedes albopictus (Diptera: Culicidae) in the United Kingdom. Journal of Vector Ecology 31: 292-304.

Medlock, J.M., K.M. Hansford, F. Schaffner, V. Versteirt, G. Hendrickx, H. Zeller, and W. Van Bortel. 2012. A review of the invasive mosquitoes in Europe: ecology, public health risks, and control options. Vector Borne and Zoonotic Diseases 12:435-447.

Medlock, J.M., K.M. Hansford, V. Versteirt, B. Cull, H. Kampen, D. Fontenille, G. Hendrickx, H. Zeller, W. Van Bortel, and F. Schaffner. 2015. An entomological review of invasive mosquitoes in Europe. Bulletin of Entomological Research 105:637663.

Melaun, C., A. Werblow, S. Cunze, S. Zotzmann, L.K. Koch, H. Mehlhorn, D.D. Dorge, K. Huber, O. Tackenberg, and S. Klimpel. 2015. Modeling of the putative 
distribution of the arbovirus vector Ochlerotatus japonicus japonicus (Diptera: Culicidae) in Germany. Parasitology Research 114: 1051-1061.

Metz, M., D. Rocchini, and M. Neteler. 2014. Surface temperatures at the continental scale: tracking changes with remote sensing at unprecedented detail. Remote Sensing 6:3822.

Miller, J. 2010. Species distribution modeling. Geography Compass 4:490-509.

Miquel, M., R. Del Río, D. Borrás, C. Barceló, C. Paredes-Esquivel, J. Lucientes, and M.A. Miranda. 2013. First detection of Aedes albopictus (Diptera: Culicidae) in the Balearic Islands (Spain). Journal of the European Mosquito Control Association 31:811.

Mitchell, C.J., M.L. Niebylski, G.C. Smith, N. Karabatsos, D. Martin, J.P. Mutebi, G.B. Craig, Jr., and M.J. Mahler. 1992. Isolation of eastern equine encephalitis virus from Aedes albopictus in Florida. Science 257: 526-527.

MODIS. 2012. www.modis.gsfc.nasa.gov.

Mohlmann, T.W.R., U. Wennergren, M. Talle, G. Favia, C. Damiani, L. Bracchetti, and C.J.M. Koenraadt. 2017. Community analysis of the abundance and diversity of mosquito species (Diptera: Culicidae) in three European countries at different latitudes. Parasites \& Vectors 10:510.

Montarsi, F., S. Martini, M. Dal Pont, N. Delai, N. Ferro Milone, M. Mazzucato, F. Soppelsa, L. Cazzola, S. Cazzin, S. Ravagnan, S. Ciocchetta, F. Russo, and G. Capelli. 2013. Distribution and habitat characterization of the recently introduced invasive mosquito Aedes koreicus [Hulecoeteomyia koreica], a new potential vector and pest in north-eastern Italy. Parasites \& Vectors 6:292.

Moustiquetigre.org. 2018. Bilan des communes colonisées par Aedes albopictus France métropolitaine - Janvier 2017. http://www.moustiquetigre.org/images/cartes/ National_Communes_2017.jpg.

MUM. 2016. MUM, Mechanical Unload System. http://www.schiphol.nl/SchipholGroup1/ NieuwsPers/Persbericht/SchipholIntroducesInnovativeUnloadingSystemForBaggageCo ntainers.html.

National Weather Service Climate Prediction Center. www.cpc.ncep.noaa.gov.

Neteler, M., D. Roiz, D. Rocchini, C. Castellani, and A. Rizzoli. 2011. Terra and Aqua satellites track tiger mosquito invasion: modelling the potential distribution of Aedes albopictus in north-eastern Italy. International Journal of Health Geographics 10: 49.

Neteler, M., M. Metz, D. Rocchini, A. Rizzoli, E. Flacio, L. Engeler, V. Guidi, P. Luthy, and M. Tonolla. 2013. Is Switzerland suitable for the invasion of Aedes albopictus? PLoS One 8: e82090.

Niebylski, M.L., and G.B. Craig, Jr. 1994. Dispersal and survival of Aedes albopictus at a scrap tire yard in Missouri. Journal of the American Mosquito Control Association 10: 339-343.

NVWA. 2016. Archief vondsten https://www.nvwa.nl/onderwerpen/muggen-knutten-enteken/archief-vondsten.

NVWA. 2017. NVWA treft tijgermug aan op bloemenveiling naaldwijk. https://www.nvwa.nl/nieuws-en-media/nieuws/2017/06/07/nvwa-treft-tijgermug-aanop-bloemenveiling-naaldwijk 2017.

NVWA. 2018. Archief vondsten exotische muggen. https://www.nvwa.nl/onderwerpen/ muggen-knutten-en-teken/archief-vondsten.

O'Donnell, K.L., M.A. Bixby, K.J. Morin, D.S. Bradley, and J.A. Vaughan. 2017. Potential of a northern population of Aedes vexans (Diptera: Culicidae) to transmit Zika Virus. Journal of Medical Entomology 54: 1354-1359.

Openflights.org. 2016. Openflights.org database. http://openflights.org/data.html.

Oracle Corporation. 2009. Oracle application express (APEX). http://apex.oracle.com. 
Osborne, P., J. Alonso, and R. Bryant. 2001. Modelling landscape-scale habitat use using GIS and remote sensing: a case study with great bustards. Journal of applied ecology 38: 458-471.

Osorio, H.C., L. Ze-Ze, M. Neto, S. Silva, F. Marques, A.S. Silva, and M.J. Alves. 2018. Detection of the invasive mosquito species Aedes (Stegomyia) albopictus (Diptera: Culicidae) in Portugal. International Journal of Environmental Research and Public Health 15: E820.

Palmer, J.R.B., A. Oltra, F. Collantes, J.A. Delgado, J. Lucientes, S. Delacour, M. Bengoa, R. Eritja, and F. Bartumeus. 2017. Citizen science provides a reliable and scalable tool to track disease-carrying mosquitoes. Nature Communications 8:916.

Papa, A. 2013. West Nile virus infections in humans - Focus on Greece. Journal of Clinical Virology 58:351-353.

Patz, J.A., H. Frumkin, T. Holloway, D.J. Vimont, and A. Haines. 2014. Climate change: challenges and opportunities for global health. Journal of the American Medical Association 312:1565-1580.

Paupy, C., H. Delatte, L. Bagny, V. Corbel, and D. Fontenille. 2009. Aedes albopictus, an arbovirus vector: from the darkness to the light. Microbes and Infection 11:1177-1185.

Peters, J., B.D. Baets, N.E.C. Verhoest, R. Samson, S. Degroeve, P.D. Becker, and W. Huybrechts. 2007. Random forests as a tool for ecohydrological distribution modelling. Ecological Modelling 207: 304-318.

Petrić, D., I. Pajović, A. Ignjatović Ćupina, and M. Zgomba. 2001. Aedes albopictus (Skuse, 1894) new mosquito species (Diptera, Culicidae) in entomofauna of Yugoslavia. Abstract of Sym posia of the entom ologists of Serbia, Goč, p.2627.

Petric, D., R. Bellini, E.J. Scholte, L.M. Rakotoarivony, and F. Schaffner. 2014. Monitoring population and environmental parameters of invasive mosquito species in Europe. Parasites \& Vectors 7:187.

Phillips, S.J., M. Dud, and R.E. Schapire. 2004. A maximum entropy approach to species distribution modeling. Proceedings of the twenty-first international conference on Machine learning. ACM, Banff, Alberta, Canada pp. 83.

Phillips, S.J., R.P. Anderson, and R.E. Schapire. 2006. Maximum entropy modeling of species geographic distributions. Ecological Modelling 190:231-259.

Piry, S., A. Alapetite, J.M. Cornuet, D. Paetkau, L. Baudouin, and A. Estoup. 2004. GENECLASS2: a software for genetic assignment and first-generation migrant detection. Journal of Heredity 95:536-539.

Pluskota, B., V. Storch, T. Braunbeck, M. Beck, and N. Becker. 2008. First record of Stegomyia albopicta (Skuse) (Diptera: Culicidae) in Germany. European Mosquito Bulletin 26:1-5.

Preacher, K.J., and A.F. Hayes. 2004. SPSS and SAS procedures for estimating indirect effects in simple mediation models. Behavior Research Methods, Instruments, \& Computers 36: 717-731.

Prioteasa, L.F., S. Dinu, E. Falcuta, and C.S. Ceianu. 2015. Established population of the invasive mosquito species Aedes albopictus in Romania, 2012-14. Journal of the American Mosquito Control Association 31:177-181.

Proestos, Y., G.K. Christophides, K. Erguler, M. Tanarhte, J. Waldock, and J. Lelieveld. 2015. Present and future projections of habitat suitability of the Asian tiger mosquito, a vector of viral pathogens, from global climate simulation. Philosophical transactions of the Royal Society of London. Series B, Biological sciences 370.

Puggioli, A., P. Bonilauri, M. Calzolari, D. Lelli, M. Carrieri, S. Urbanelli, D. Pudar, and R. Bellini. 2017. Does Aedes albopictus (Diptera: Culicidae) play any role in 
Usutu virus transmission in Northern Italy? Experimental oral infection and field evidences. Acta Tropica 172:192-196.

QGIS Development Team. 2012. QGIS Geographic Information System. Open Source Geospatial Foundation Project. http://qgis.osgeo.org.

Ramasamy, R., and S.N. Surendran. 2012. Global climate change and its potential impact on disease transmission by salinity-tolerant mosquito vectors in coastal zones. Frontiers Physiology 3: 198.

Ramchurn, S.K., K. Moheeput, and S.S. Goorah. 2009. An analysis of a short-lived outbreak of dengue fever in Mauritius. Eurosurveillance 14:19314.

Randolph, S.E., and D.J. Rogers. 2010. The arrival, establishment and spread of exotic diseases: patterns and predictions. Nature Reviews Microbiology 8:361-371.

Rannala, B., and J.L. Mountain. 1997. Detecting immigration by using multilocus genotypes. Proceedings of the National Academy of Sciences of the United States of America 94: 9197-9201.

Ravanini, P., E. Huhtamo, V. Ilaria, M.G. Crobu, A. M. Nicosia, L. Servino, F. Rivasi, S. Allegrini, U. Miglio, A. Magri, R. Minisini, O. Vapalahti, and R. Boldorini. 2012. Japanese encephalitis virus RNA detected in Culex pipiens mosquitoes in Italy. Eurosurveillance 17:20221.

Ravasi, D., V. Guidi, E. Flacio, P. Luthy, K. Perron, S. Ludin, and M. Tonolla. 2018. Investigation of temperature conditions in Swiss urban and suburban microhabitats for the overwintering suitability of diapausing Aedes albopictus eggs. Parasites \& Vectors $11: 212$.

Reiter, P. 2010. Yellow fever and dengue: a threat to Europe? Eurosurveillance 15:19509.

Reiter, P., and D. Sprenger. 1987. The used tire trade: a mechanism for the worldwide dispersal of container breeding mosquitoes. Journal of the American Mosquito Control Association 3:494-501.

Rezza, G., L. Nicoletti, R. Angelini, R. Romi, A.C. Finarelli, M. Panning, P. Cordioli, C. Fortuna, S. Boros, F. Magurano, G. Silvi, P. Angelini, M. Dottori, M.G. Ciufolini, G.C. Majori, and A. Cassone. 2007. Infection with chikungunya virus in Italy: an outbreak in a temperate region. The Lancet 370: 1840-1846.

Rich, K.M., and F. Wanyoike. 2010. An assessment of the regional and national socioeconomic impacts of the 2007 Rift Valley fever outbreak in Kenya. The American Journal of Tropical Medicine and Hygiene 83:52-57.

Rijks, J.M., M. Kik, M.G. Koene, M.Y. Engelsma, P. van Tulden, M.G. Montizaan, T. Oomen, M.A. Spierenburg, J. Ijzer, J.W. van der Giessen, A. Grone, and H.J. Roest. 2013. Tularaemia in a brown hare (Lepus europaeus) in 2013: first case in the Netherlands in 60 years. Eurosurveillance 18:20655.

Rijks, J.M., M.L. Kik, R. Slaterus, R. Foppen, A. Stroo, J. Ijzer, J. Stahl, A. Grone, M. Koopmans, H.P. van der Jeugd, and C. Reusken. 2016. Widespread Usutu virus outbreak in birds in the Netherlands, 2016. Eurosurveillance 21:30391.

Rogers, D.J. 2000. Satellites, space, time and the African trypanosomiases. Advances in Parasitology 47:129-171.

Rogers, D.J. 2006. Models for vectors and vector-borne diseases. Advances in Parasitology 62:1-35.

Rogers, D.J., and B.G. Williams. 1994. Tsetse distribution in Africa: seeing the wood and the trees. In: Edwards, P.J., R.M. May, and N. Webb (Eds.), Large -Scale Ecology and Conservation Biology. Oxford, UK: Blackwell Scientific 247-271.

Rogers, D.J., and S.E. Randolph. 2006. Climate change and vector-borne diseases. Advances in Parasitology 62:345-381. 
Roiz, D., M. Neteler, C. Castellani, D. Arnoldi, and A. Rizzoli. 2011. Climatic factors driving invasion of the tiger mosquito (Aedes albopictus) into new areas of Trentino, northern Italy. PLoS One 6:e14800.

Roiz, D., R. Eritja, R. Melero-Alcibar, R. Molina, E. Marquès, S. Ruiz, R. Escosa, C. Aranda, and J. Lucientes. 2007. Distribución de Aedes (Stegomyia) albopictus (Skuse, 1894) (Diptera: Culicidae) en España. Boletín de la SEA - Sociedad Entomológica Aragonesa 40:523-526.

Roiz, D., S. Duperier, M. Roussel, P. Bousses, D. Fontenille, F. Simard, and C. Paupy. 2016. Trapping the tiger: efficacy of the novel BG-Sentinel 2 with several attractants and carbon dioxide for collecting Aedes albopictus (Diptera: Culicidae) in Southern France. Journal of Medical Entomology 53:460-465.

Romi, R., G. Sabatinelli, L.G. Savelli, M. Raris, M. Zago, and R. Malatesta. 1997. Identification of a North American mosquito species, Aedes atropalpus (Diptera: Culicidae), in Italy. Journal of the American Mosquito Control Association 13: 245246.

Rudolf, I., H. Blazejova, P. Strakova, O. Sebesta, J. Pesko, J. Mendel, S. Sikutova, Z. Hubalek, H. Kampen, and F. Schaffner. 2018. The invasive Asian tiger mosquito Aedes albopictus (Diptera: Culicidae) in the Czech Republic: Repetitive introduction events highlight the need for extended entomological surveillance. Acta Tropica 185: 239-241.

Rudolf, M., C. Czajka, J. Borstler, C. Melaun, H. Jost, H. von Thien, M. Badusche, N. Becker, J. Schmidt-Chanasit, A. Kruger, E. Tannich, and S. Becker. 2013. First nationwide surveillance of Culex pipiens complex and Culex torrentium mosquitoes demonstrated the presence of Culex pipiens biotype pipiens/molestus hybrids in Germany. PLoS One 8:e71832.

Sabatini, A., V. Raineri, G. Trovato, and M. Coluzzi. 1990. Aedes albopictus in Italy and possible diffusion of the species into the Mediterranean area. Parassitologia 32: 301304.

Samanidou-Voyadjoglou, A., E. Patsoula, G. Spanakos, and N.C. Vakalis. 2005. Confirmation of Aedes albopictus (Skuse) (Diptera: Culicidae) in Greece. European Mosquito Bulletin 19: 10-12.

Sambri, V., M. Capobianchi, R. Charrel, M. Fyodorova, P. Gaibani, E. Gould, M. Niedrig, A. Papa, A. Pierro, G. Rossini, S. Varani, C. Vocale, and M.P. Landini. 2013. West Nile virus in Europe: emergence, epidemiology, diagnosis, treatment, and prevention. Clinical Microbiology and Infection 19: 699-704.

Sardelis, M.R., and M.J. Turell. 2001. Ochlerotatus j. japonicus in Frederick County, Maryland: discovery, distribution, and vector competence for West Nile virus. Journal of the American Mosquito Control Association 17:137-141.

Sardelis, M.R., M.J. Turell, and R.G. Andre. 2002a. Laboratory transmission of La Crosse virus by Ochlerotatus j. japonicus (Diptera: Culicidae). Journal of Medical Entomology 39:635-639.

Sardelis, M.R., D.J. Dohm, B. Pagac, R.G. Andre, and M.J. Turell. 2002b. Experimental transmission of eastern equine encephalitis virus by Ochlerotatus j. japonicus (Diptera: Culicidae). Journal of Medical Entomology 39:480-484.

Sardelis, M.R., M.J. Turell, and R.G. Andre. 2003. Experimental transmission of St. Louis encephalitis virus by Ochlerotatus j. japonicus. Journal of the American Mosquito Control Association 19:159-162.

Schaffner, F., and A. Mathis. 2014. Dengue and dengue vectors in the WHO European region: past, present, and scenarios for the future. The Lancet Infectious Diseases 14:1271-1280. 
Schaffner, F., and S. Karch. 2000. First report of Aedes albopictus (Skuse, 1984) in metropolitan France. Comptes Rendus de l'Académie des Sciences - Series III 323:373375.

Schaffner, F., C. Kaufmann, D. Hegglin, and A. Mathis. 2009. The invasive mosquito Aedes japonicus in Central Europe. Medical and Veterinary Entomology 23: 448-451.

Schaffner, F., G. Angel, B. Geoffroy, J.P. Hervy, A. Rhaiem, and J. Brunhes. 2001. The mosquitoes of Europe: An identification and training programme. IRD Editions \& EID Méditerranée CD-ROM, Montpellier, France.

Schaffner, F., I. Thiery, C. Kaufmann, A. Zettor, C. Lengeler, A. Mathis, and C. Bourgouin. 2012. Anopheles plumbeus (Diptera: Culicidae) in Europe: a mere nuisance mosquito or potential malaria vector? Malaria Journal 11: 393.

Schaffner, F., J.M. Medlock, and W. Van Bortel. 2013. Public health significance of invasive mosquitoes in Europe. Clinical Microbiology and Infection 19:685-692.

Schaffner, F., M. Marquine, N. Pasteur, and M. Raymond. 2003. Genetic differentiation of Anopheles claviger s.s. in Europe. Journal of Medical Entomology 40:865-875.

Schaffner, F., M. Vazeille, C. Kaufmann, A.B. Failloux, and A. Mathis. 2011. Vector competence of Aedes japonicus for chikungunya and dengue viruses. European Mosquito Bulletin 29: 141-142.

Schaffner, F., R. Bellini, D. Petric, E.J. Scholte, H. Zeller, and L.M. Rakotoarivony. 2013. Development of guidelines for the surveillance of invasive mosquitoes in Europe. Parasites \& Vectors 6: 209.

Schaffner, F., S. Chouin, and J. Guilloteau. 2003. First record of Ochlerotatus (Finlaya) japonicus japonicus (Theobald, 1901) in metropolitan France. Journal of the American Mosquito Control Association 19:1-5.

Schaffner, F., W. Van Bortel, and M. Coosemans. 2004. First record of Aedes (Stegomyia) albopictus in Belgium. Journal of the American Mosquito Control Association 20: 201203.

Scharlemann, J.P., D. Benz, S.I. Hay, B.V. Purse, A.J. Tatem, G.R. Wint, and D.J. Rogers. 2008. Global data for ecology and epidemiology: a novel algorithm for temporal Fourier processing MODIS data. PLoS One 3: e1408.

Scholte, E.J., and F. Schaffner. 2007. Waiting for the tiger: establishment and spread of the Aedes albopictus mosquito in Europe. In: Takken, W., and B.G.J. Knols (Eds.) Emerging pests and vector-borne diseases in Europe. Wageningen, The Netherlands: 241-260.

Scholte, E.J., E. Dijkstra, H. Blok, A. De Vries, W. Takken, A. Hofhuis, M. Koopmans, A. De Boer, and C.B. Reusken. 2008. Accidental importation of the mosquito Aedes albopictus into the Netherlands: a survey of mosquito distribution and the presence of dengue virus. Medical and Veterinary Entomology 22: 352-358.

Scholte, E.J., F. Jacobs, Y.M. Linton, E. Dijkstra, J. Fransen, and W. Takken. 2007. First record of Aedes (Stegomyia) albopictus in the Netherlands. European Mosquito Bulletin 22:5-9.

Scholte, E.J., W. Den Hartog, M. Braks, C. Reusken, M. Dik, and A. Hessels. 2009. First report of a North American invasive mosquito species Ochlerotatus atropalpus (Coquillett) in the Netherlands, 2009. Eurosurveillance 14: 19400.

Scholte, E.J., W. Den Hartog, M. Dik, B. Schoelitsz, M. Brooks, F. Schaffner, R. Foussadier, M. Braks, and J. Beeuwkes. 2010a. Introduction and control of three invasive mosquito species in the Netherlands, July-October 2010. Eurosurveillance 15: 19710.

Scholte, E.J., M. Braks, and F. Schaffner. 2010b. Aircraft-mediated transport of Culex quinquefasciatus: a case report. European Mosquito Bulletin 28: 208-212. 
Scholte, E.J., W. den Hartog, and C. Reusken. 2011. A report of Anopheles algeriensis (Diptera: Culicidae) from The Netherlands. Entomologische berichten 71:39-42.

Scholte, E.J., M. Dik, A. Ibañez Justicia, W. Den Hartog, B. Schoelitsz, M. Brooks, M. Braks, and M. Steeghs. 2012. Findings and control of two invasive exotic mosquito species, Aedes albopictus and Ae. atropalpus (Diptera: Culicidae) in the Netherlands, 2011. European Mosquito Bulletin 30:1-14.

Scholte, E.J., M.H. Mars, M. Braks, W. Den Hartog, A. Ibañez-Justicia, M. Koopmans, C.J. Koenraadt, A. De Vries, and C. Reusken. 2013. No evidence for the persistence of Schmallenberg virus in overwintering mosquitoes. Medical and Veterinary Entomology 28:110-115.

Scholte, E.J., A. Ibañez-Justicia, A. Stroo, J. De Zeeuw, W. den Hartog, and C. Reusken. 2014. Mosquito collections on incoming intercontinental flights at Schiphol International airport, the Netherlands, 2010-2011. Journal of the European Mosquito Control Association 32:17-21.

Sebesta, O., I. Rudolf, L. Betasova, J. Pesko, and Z. Hubalek. 2012. An invasive mosquito species Aedes albopictus found in the Czech Republic, 2012. Eurosurveillance 17:20301.

Seidel, B., D. Duh, N. Nowotny, and F. Allerberger. 2012. Erstnachweis der Stechmücken Aedes (Ochlerotatus) japonicus japonicus (Theobald, 1901) in Österreich und Slowenien in 2011 und für Aedes (Stegomyia) albopictus (Skuse, 1895) in Österreich 2012 (Diptera: Culicidae). Entomologische Zeitschrift 122:223-226.

Seidel, B., F. Montarsi, H.P. Huemer, A. Indra, G. Capelli, F. Allerberger, and N. Nowotny. 2016b. First record of the Asian bush mosquito, Aedes japonicus japonicus, in Italy: invasion from an established Austrian population. Parasites \& Vectors 9:284.

Seidel, B., N. Nowotny, T. Bakonyi, F. Allerberger, and F. Schaffner. 2016a. Spread of Aedes japonicus japonicus (Theobald, 1901) in Austria, 2011-2015, and first records of the subspecies for Hungary, 2012, and the principality of Liechtenstein, 2015. Parasites \& Vectors 9.

Semenza, J.C., and J.E. Suk. 2018. Vector-borne diseases and climate change: a European perspective. FEMS Microbiology Letters 365, doi: 10.1093/femsle/fnx244.

Shrout, P.E., and N. Bolger. 2002. Mediation in experimental and nonexperimental studies: new procedures and recommendations. Psychological Methods 7:422-445.

Shute, P.G., and M. Maryon. 1974. Malaria in England past, present and future. Royal Society of Health Journal 94:23-29.

Sieg, M., V. Schmidt, U. Ziegler, M. Keller, D. Hoper, K. Heenemann, A. Ruckner, H. Nieper, A. Muluneh, M.H. Groschup, and T.W. Vahlenkamp. 2017. Outbreak and co-circulation of three different Usutu virus strains in Eastern Germany. Vector Borne and Zoonotic Diseases 17:662-664.

Slotman, M.A., N.B. Kelly, L.C. Harrington, S. Kitthawee, J.W. Jones, T.W. Scott, A. Caccone, and J.R. Powell. 2007. Polymorphic microsatellite markers for studies of Aedes aegypti (Diptera: Culicidae), the vector of dengue and yellow fever. Molecular Ecology Notes 7:168-171.

Snow, K.R. 1990. Naturalists'Handbooks 14: mosquitoes. Richmont Publishing Co. Ltd. Slough.

Socioeconomic Data and Applications Center. www.sedac.ciesin.columbia.edu.

Sokolova, M.I., and K.R. Snow. 2002. Malaria vectors in European Russia. European Mosquito Bulletin 12:1-6.

Sota, T., M. Mogi, and E. Hayamizu. 1994. Habitat stability and the larval mosquito community in treeholes and other containers on a temperate Island. Researches on Population Ecology 36:93-104. 
Sousa, C.A., M. Clairouin, G. Seixas, B. Viveiros, M.T. Novo, A.C. Silva, M.T. Escoval, and A. Economopoulou. 2012. Ongoing outbreak of dengue type 1 in the Autonomous Region of Madeira, Portugal: preliminary report. Eurosurveillance 17: 20333.

Spiteri, G., B. Sudre, A. Septfons, J. Beaute, and The European Zika Surveillance Network. 2017. Surveillance of Zika virus infection in the EU/EEA, June 2015 to January 2017. Eurosurveillance 22: 17-00254.

Sprenger, D., and T. Wuithiranyagool. 1986. The discovery and distribution of Aedes albopictus in Harris County, Texas. Journal of the American Mosquito Control Association 2: 217-219.

Staatsblad van het Koninkrijk der Nederlanden. 2011. Besluit van 11 april 2011, houdende regels met betrekking tot productvoorschriften voor Lucky Bamboo (Warenwetbesluit Lucky Bamboo). Staatsblad van het Koninkrijk der Nederlanden. http://wetten.overheid.nl/BWBR0029929/2016-02-19.

Staatsblad van het Koninkrijk der Nederlanden. 2013. Convenant Voorkomen of beperken van herhaalde insleep van exotische muggen bij bedrijven die handelen in gebruikte banden. https://zoek.officielebekendmakingen.nl/stcrt-2013-18303.html.

Staatsblad van het Koninkrijk der Nederlanden. 2014. Besluit van 5 september 2014, houdende wijziging van het Warenwetbesluit Lucky Bamboo in verband met een wijziging van de definitie van Lucky Bamboo en de uitbreiding van de reikwijdte van het besluit tot invoer uit EU-landen. https://zoek.officielebekendmakingen.nl/stb-2014338.html.

Staatsblad van het Koninkrijk der Nederlanden. 2016. Convenant Voorkomen of beperken van insleep van exotische muggen bij bedrijven die handelen in gebruikte banden. https://zoek.officielebekendmakingen.nl/stcrt-2016-35197.html.

Suter, T., E. Flacio, B. F. Farina, L. Engeler, M. Tonolla, and P. Muller. 2015. First report of the invasive mosquito species Aedes koreicus in the Swiss-Italian border region. Parasit Vectors 8: 402.

Swellengrebel, N.H., A. de Buck, and H. Kraan. 1938. Mechanism of malaria transmission in the province of North Holland. Journal of Hygiene 38:62-74.

Tabachnick, W.J. 1991. Evolutionary genetics and arthropod-borne disease: The yellow fever mosquito. American Entomologist 37:14-26.

Takashima, I., and L. Rosen. 1989. Horizontal and vertical transmission of Japanese encephalitis virus by Aedes japonicus (Diptera: Culicidae). Journal of Medical Entomology 26:454-458.

Takken, W., P.A. Kager, and J.P. Verhave. 2007. Will malaria return to North-West Europe?, pp. 23-24. In W. Takken and B.G.J. Knols (Eds). Emerging pests and vectorborne diseases in Europe. Wageningen, The Netherlands.

Takken, W., R. Geene, W. Adam, T.H. Jetten, and J.A. van der Velden. 2002. Distribution and dynamics of larval populations of Anopheles messeae and $A$. atroparvus in the delta of the rivers Rhine and Meuse, The Netherlands. Ambio 31: 212-218.

Takumi, K., E.J. Scholte, M. Braks, C. Reusken, D. Avenell, and J.M. Medlock. 2009. Introduction, scenarios for establishment and seasonal activity of Aedes albopictus in The Netherlands. Vector Borne and Zoonotic Diseases 9:191-196.

Tanaka, K., K. Mizusawa, and E.S. Saugstad. 1979. A revision of the adult and larval mosquitoes of Japan (including the Ryukyu archipelago and the Ogasawara islands) and Korea (Diptera: Culicidae). Contributions of the American Entomological Institute 16:1-987.

Tarantola, A., F. Goutard, P. Newton, X. de Lamballerie, O. Lortholary, J. Cappelle, and P. Buchy. 2014. Estimating the burden of Japanese encephalitis virus and other 
encephalitides in countries of the mekong region. PLoS Neglected Tropical Diseases 8:e2533.

Tatem, A.J., D.J. Rogers, and S.I. Hay. 2006. Estimating the malaria risk of African mosquito movement by air travel. Malaria Journal 5:57.

Tatem, A.J., S.I. Hay, and D.J. Rogers. 2006. Global traffic and disease vector dispersal. Proceedings of the National Academy of Sciences of the United States of America 103:6242-6247.

Thomas, S.M., U. Obermayr, D. Fischer, J. Kreyling, and C. Beierkuhnlein. 2012. Lowtemperature threshold for egg survival of a post-diapause and non-diapause European aedine strain, Aedes albopictus (Diptera: Culicidae). Parasites \& Vectors 5:100.

Toma, L., F. Toma, G. Pampiglione, M. Goffredo, F. Severini, and M. Di Luca. 2017. First record of Aedes albopictus (Skuse, 1894) (Diptera; Culicidae) from three islands in the Tyrrhenian Sea (Italy). Journal of the European Mosquito Control Association 35:25-28.

Townroe, S., and A. Callaghan. 2014. British container breeding mosquitoes: the impact of urbanisation and climate change on community composition and phenology. PLoS One 9:e95325.

Turell, M.J., W.C. Wilson, and K.E. Bennett. 2010. Potential for North American mosquitoes (Diptera: Culicidae) to transmit rift valley fever virus. Journal of Medical Entomology 47:884-889.

Unlu, I., A. Farajollahi, D. Strickman, and D.M. Fonseca. 2013. Crouching tiger, hidden trouble: urban sources of Aedes albopictus (Diptera: Culicidae) refractory to sourcereduction. PLoS One 8:e77999.

Unlu, I., and A. Farajollahi. 2014. A multiyear surveillance for Aedes albopictus with Biogents Sentinel trap counts for males and species composition of other mosquito species. Journal of the American Mosquito Control Association 30:122-125.

VACO Bedrijfstakorganisatie voor de banden- en wielenbranche. 2016. http://www.vaco.nl/.

Vallorani, R., P. Angelini, R. Bellini, M. Carrieri, A. Crisci, S. Mascali Zeo, G. Messeri, and C. Venturelli. 2015. Temperature characterization of different urban microhabitats of Aedes albopictus (Diptera Culicidae) in Central-Northern Italy. Environmental Entomology 44:1182-1192.

van de Vossenberg, B.T., A. Ibañez-Justicia, E. Metz-Verschure, E.J. van Veen, M.L. Bruil-Dieters, and E.J. Scholte. 2015. Real-time PCR Tests in Dutch exotic mosquito surveys; Implementation of Aedes aegypti and Aedes albopictus identification tests, and the development of tests for the identification of Aedes atropalpus and Aedes japonicus japonicus (Diptera: Culicidae). Journal of Medical Entomology 52:336-350.

van Haren, J.C.M. and P.F.M. Verdonschot. 1995. Proeftabel Nederlandse Culicidae. Rapport van het Instituut voor Bos- en Natuurbeheer Wageningen 173:1-106: 106p.

van Seventer, H.A. 1969. The disappearance of malaria in the Netherlands. Nederlands Tijdschrift voor Geneeskunde 113:2055-2056.

Vazquez-Prokopec, G.M., L.F. Chaves, S.A. Ritchie, J. Davis, and U. Kitron. 2010. Unforeseen costs of cutting mosquito surveillance budgets. PLoS Neglected Tropical Diseases 4:e858.

Velo, E., P. Kadriaj, K. Mersini, A. Shukullari, B. Manxhari, A. Simaku, A. Hoxha, B. Caputo, L. Bolzoni, R. Rosa, S. Bino, P. Reiter, and A. della Torre. 2016. Enhancement of Aedes albopictus collections by ovitrap and sticky adult trap. Parasites \& Vectors 9:223.

Venturi, G., M. Di Luca, C. Fortuna, M.E. Remoli, F. Riccardo, F. Severini, L. Toma, M. Del Manso, E. Benedetti, M.G. Caporali, A. Amendola, C. Fiorentini, C. De 
Liberato, R. Giammattei, R. Romi, P. Pezzotti, G. Rezza, and C. Rizzo. 2017. Detection of a chikungunya outbreak in Central Italy, August to September 2017. Eurosurveillance 22: 17-00646.

Verdonschot, P.F.M. 2002. Culicidae. In: Beuk (2002) Checklist of the Diptera of the Netherlands. KNNV, Uitgeverij, Utrecht: 98-100.

Verdonschot, P.F.M., and A.A. Besse-Lototskaya. 2014. Flight distance of mosquitoes (Culicidae): A metadata analysis to support the management of barrier zones around rewetted and newly constructed wetlands. Limnologica - Ecology and Management of Inland Waters 45:69-79.

Veronesi, R., M. Carrieri, B. Maccagnani, S. Maini, and R. Bellini. 2015. Macrocyclops albidus (Copepoda: cyclopidae) for the Biocontrol of Aedes albopictus and Culex pipiens in Italy. Journal of the American Mosquito Control Association 31:32-43.

Versteirt, V., E. De Clercq, W. Dekoninck, D. Damiens, A. Ayrinhac, F. Jacobs, and W. Van Bortel. 2009. Mosquito vectors of disease: spatial biodiversity, drivers of change, and risk "MODIRISK" (Research Program m e Science for a Sustainable Developm ent). Final Report. Brussels: Belgian Science Policy 2009 - 152 p.

Versteirt, V., E.M. De Clercq, D.M. Fonseca, J. Pecor, F. Schaffner, M. Coosemans, and W. Van Bortel. 2012. Bionomics of the established exotic mosquito species Aedes koreicus in Belgium, Europe. Journal of Medical Entomology 49:1226-1232.

Versteirt, V., F. Schaffner, C. Garros, W. Dekoninck, M. Coosemans, and W. Van Bortel. 2009. Introduction and establishment of the exotic mosquito species Aedes japonicus japonicus (Diptera: Culicidae) in Belgium. Journal of Medical Entomology 46:1464-1467.

Versteirt, V., S. Boyer, D. Damiens, E.M. De Clercq, W. Dekoninck, E. Ducheyne, P. Grootaert, C. Garros, T. Hance, G. Hendrickx, M. Coosemans, and W. Van Bortel. 2013. Nationwide inventory of mosquito biodiversity (Diptera: Culicidae) in Belgium, Europe. Bulletin of entomological research 103: 193-203.

Vogels, C.B., L.J. van de Peppel, A.J. van Vliet, M. Westenberg, A. Ibañez-Justicia, A. Stroo, J.A. Buijs, T.M. Visser, and C.J. Koenraadt. 2015. Winter activity and aboveground hybridization between the two biotypes of the West Nile virus vector Culex pipiens. Vector Borne and Zoonotic Diseases 15:619-626.

Walther, D., D.E. Scheuch, and H. Kampen. 2017. The invasive Asian tiger mosquito Aedes albopictus (Diptera: Culicidae) in Germany: Local reproduction and overwintering. Acta Tropica 166:186-192.

Weissenbock, H., J. Kolodziejek, K. Fragner, R. Kuhn, M. Pfeffer, and N. Nowotny. 2003. Usutu virus activity in Austria, 2001-2002. Microbes and Infection 5: 1132-1136.

Werner, D., and H. Kampen. 2013. The further spread of Aedes japonicus japonicus (Diptera, Culicidae) towards northern Germany. Parasitology Research 112:3665-3668.

Werner, D., and H. Kampen. 2015. Aedes albopictus breeding in southern Germany, 2014. Parasitology Research 114:831-834.

Werner, D., D.E. Zielke, and H. Kampen. 2016. First record of Aedes koreicus (Diptera: Culicidae) in Germany. Parasitology Research 115: 1331-1334.

Werner, D., M. Kronefeld, F. Schaffner, and H. Kampen. 2012. Two invasive mosquito species, Aedes albopictus and Aedes japonicus japonicus, trapped in south-west Germany, July to August 2011. Eurosurveillance 17: 20067

Westby, K.M., C. Fritzen, D. Paulsen, S. Poindexter, and A.C. Moncayo. 2015. La Crosse encephalitis virus infection in field-collected Aedes albopictus, Aedes japonicus, and Aedes triseriatus in Tennessee. Journal of the American Mosquito Control Association 31:233-241. 
Westcott, R., K. Ronan, H. Bambrick, and M. Taylor. 2017. Expanding protection motivation theory: investigating an application to animal owners and emergency responders in bushfire emergencies. BMC Psychology 5:13.

Whelan, P., H. Nguyen, K. Hajkowicz, J. Davis, D. Smith, A. Pyke, V. Krause, and P. Markey. 2012. Evidence in Australia for a case of airport dengue. PLoS Neglected Tropical Diseases 6: e1619.

Whittaker, R.H. 1972. Evolution and measurement of species diversity. Taxon 21:213-251.

WHO. 1995. International Programme on Chemical Safety (1995) Report of the informal consultation on aircraft disinsection. World Health Organization. 57p.

WHO. 2017. Global vector control response 2017-2030, WHO World Health Organisation. http://www.who.int/vector-control/publications/global-control-response/en/.

WorldClim. 2018. www.worldclim.org.

Yee, D.A. 2008. Tires as habitats for mosquitoes: a review of studies within the eastern United States. Journal of Medical Entomology 45:581-593.

Zhao, L., X. Lee, R.B. Smith, and K. Oleson. 2014. Strong contributions of local background climate to urban heat islands. Nature 511:216-219.

Zielke, D.E., A. Ibañez-Justicia, K. Kalan, E. Merdic, H. Kampen, and D. Werner. 2015. Recently discovered Aedes japonicus japonicus (Diptera: Culicidae) populations in The Netherlands and northern Germany resulted from a new introduction event and from a split from an existing population. Parasites \& Vectors 8: 40. 

Summary 
The collection of spatiotemporal data on mosquito vectors is crucial to understand the risk of mosquito-borne diseases that an area can face. In case of an outbreak of a newly introduced mosquito-borne pathogen of medical or veterinary importance, a reliable and updated distribution map of established populations of all mosquito species (indigenous and exotic) that can serve as vectors of the pathogens is required. It is also needed to know when and roughly in which numbers, these vectors occur. As the presence of the mosquito vector is a basic requirement for pathogen transmission to occur, the higher the abundance of pathogeninfected vectors is, the higher will be the risk of disease transmission. Vector abundance is especially important in emerging epidemics because high vector populations (relative to host abundance) result in a high basic reproduction number $\left(\mathrm{R}_{0}\right)$ of the pathogen, and as consequence, a high $\mathrm{R}_{0}$ will result in a greater ability of a disease to persist in a susceptible host population. With the vector information, and information of other factors that can affect disease transmission (e.g. host abundance, environmental conditions), it could be possible to assess which areas could be at high or low risk for a specific disease. This should be the focus areas for the authorities charged with the prevention and control of infectious disease. Where necessary, preventive or control measures could and should be taken. This thesis focused on the development of methodologies for acquiring accurate information on the actual and potential spatio-temporal distribution of indigenous and exotic mosquito species in the Netherlands, and the evaluation of the surveillance and control methodologies applied. Results of a surveillance of mosquito species in the Netherlands, conducted from 2010 by the Centre for Monitoring of Vectors (CMV) of the National Reference Centre (NRC) of the Netherlands Food and Consumer Product Safety Authority (NVWA), were evaluated.

The rationale for the study was the increasingly frequent reporting of invasive mosquito species (IMS) in the Netherlands, as well as the lack of detailed knowledge on the spatiotemporal distribution of the indigenous mosquito fauna. In the recent decade, several exotic mosquito species have become established in European countries, and further expansion of their distribution into the Netherlands seemed likely. It was therefore decided to investigate the current situation of potential disease vectors that are indigenous, as well as the invasive threat posed by exotic mosquito-borne disease vectors. At the start of the study, a survey was conducted to establish a baseline for the spatio-temporal distribution of the indigenous mosquito fauna present in the Netherlands (Chapter 2). In the study, cross-sectional mosquito field surveys were carried out in more than 700 locations over a period of four years (2010-2013). The data obtained were analysed and presented in terms of species 
composition in three habitat types (urban, agricultural and natural), geographical distribution of the species over the country, and seasonality. Mosquito species diversity of the three habitats was calculated using richness and evenness indices. This survey provided occurrence maps for 26 indigenous mosquito species and one invasive species (Aedes japonicus). This strategy is well suited to not only better understand the distribution of the indigenous mosquito fauna but also as a technique for detection of hitherto unknown populations of established IMS. Mapping the distribution of mosquito species using this strategy is strongly recommended as a routine surveillance for native mosquitoes.

Using the data on occurrence and abundance obtained in Chapter 2, a study on the potential spatial distribution and population density of Anopheles plumbeus, a native nuisance mosquito species, was carried out to confirm reports that this species is increasing in density as well as geographic distribution (Chapter 3). Random forest models were used to link the occurrence and abundance of An. plumbeus with environmental features obtained from satellite imagery and weather. Spatio-temporal mosquito data were provided by the four-year cross-sectional study performed in Chapter 2. For the validation of the occurrence model, data on An. plumbeus presence from confirmed nuisance notifications and data from other mosquito surveys carried out by the CMV during the years 2010-2014 were used. I found a high environmental suitability and abundance of this species in the south-eastern provinces of Limburg and Brabant. These areas were mostly associated with abandoned pig farm buildings and coincide with the areas where in recent years most nuisance has been reported.

The identification of pathways for introduction of IMS is critical for monitoring teams and decision makers at national and regional level within Europe, to decide on surveillance strategies needed to reduce the risk of future introductions and/or potential outbreaks of mosquito-borne diseases. In Chapter 4, the current knowledge of pathways associated with the introduction and dispersal of IMS in Europe was reviewed. Four main routes for IMS introduction and dispersal were identified and discussed: the trade in used tires, the import of Lucky bamboo plants from Asia, the passive transport of IMS in vehicles (traffic by road, airplanes, and sea), and the natural dispersal of IMS. Here I found that the trade in used tires remains the main pathway for long distance transportation of IMS into and within Europe, and that passive transport in ground vehicles represented the major driving force for dispersion from already established IMS populations.

For effective detection of IMS, risk-based surveillance needs to consider all likely introduction pathways for IMS. The study presented in Chapter $\mathbf{5}$ was set out to identify the 
European m osquito experts' perception of risk for introduction of IMS via different known documented pathways (from Chapter 4), to investigate what drives experts' attitudes towards support of taking actions against introduction of IMS and to investigate if other, not previously documented introduction pathways are present. For this purpose, a questionnaire to collect expert data was distributed among participants of the EMCA (European Mosquito Control Association) conference in Montenegro in March 2017. This study shows that there were no pathways unknown to the European experts that could lead to cryptic introductions at the experts' countries. Furthermore, I demonstrated that the perceived efficacy of the surveillance and control is key for the experts to overcome the experienced constraints and to support the implementation of actions against introductions. Results showed that ground transportation (with cars, trucks, etc.), passive natural dispersal, and used-tire shipments are perceived as the most likely pathways for introduction and dispersal of IMS in Europe. Introduction via aircraft appeared less well known and was not perceived probable.

Apart from gaining information on the spatial distribution of mosquito species in a given area, risk-based surveillance of IMS is key for prompt detection of introductions at risk locations, allowing appropriate and rapid implementation of control measures. In the next three Chapters (6, 7 and 8) I analyzed the results of risk-based IMS surveillance, and their management and control in the Netherlands. The detection of Aedes albopictus in Lucky bamboo greenhouses in 2005 and Ae. atropalpus at used tire importers in 2009, illustrates that the Netherlands is exposed to the risk of introductions of IMS. Chapter 6 presents the results (2010-2016) of a risk-based and adaptive surveillance of IMS implemented in Lucky bamboo greenhouses and used tires companies, with a focus on the effectiveness of the surveillance methodology, management and control operations. In addition, a revised methodology to assign used tire companies to different risk categories for the import of IMS is presented. Results show that import of Lucky bamboo introduced Ae. albopictus every year since 2010, and for the first time, Ae. japonicus was associated with the import of this plant species as well. At used tire companies, IMS identified were Ae. albopictus, Ae. atropalpus, Ae. aegypti, and Ae. japonicus. The study concludes that risk-based and adaptive surveillance is an effective methodology for the detection of IMS, and the application of governmental management measures in combination with mosquito control has kept introductions under control at these locations.

Introduction of exotic mosquitoes at Schiphol airport in the Netherlands has been considered plausible based on earlier findings of mosquitoes in aircraft cabins during CMV surveillance. 
Current surveillance efforts at Schiphol have focused on prompt detection of accidental introductions at the airport facilities using mosquito traps deployed at the most likely locations for arrival of the invasive Aedes mosquitoes. The aim of this surveillance was to quickly react and avoid temporary proliferation or establishment of mosquito populations, identify the origin of the introductions, and prevent potential transmission of vector-borne diseases. In Chapter 7, the results of surveillance conducted at the international airport of Schiphol are presented. In the study, adult yellow fever mosquitoes (Ae. aegypti) were captured indoors and outdoors at the airport of Schiphol confirming, for the first time, transport of this mosquito vector species via aircrafts into Europe. Shipments of containers carried by aircrafts are considered the most likely pathway for this introduction. GIS analysis and genetic assignment tests of these mosquitoes point to North America or the Middle East as possible origins, but the small sample size prevented us from reliably identifying the geographic origin of this introduction.

The effectiveness of mosquito control on reducing populations of an established IMS (Ae. japonicus) in the Netherlands was studied in Chapter 8. A targeted mosquito control campaign began in 2015 in the municipality of Lelystad with the objective of reducing populations in locations with the highest concentrations of Ae. japonicus breeding sites: allotment garden complexes. Mosquito control consisted of source reduction combined with application of larvicides in breeding sites. At eight complexes, mosquito control effectiveness was systematically measured by sampling larvae from breeding sites. Six measurements were performed between 2015 and 2016. Results show that the effectiveness of mosquito control actions was similar in all treated allotment gardens, and resulted in a significant reduction in Ae. japonicus larval abundance. Rain barrels at the allotments represent the most frequent breeding site, but each water filled artificial container is a potential breeding site for the species. Aedes japonicus was not found in the samples taken in other allotment gardens outside the municipality; however, the collection methodology was effective in detecting this species when it has newly colonized surrounding areas.

In the Netherlands, Ae. albopictus has been found outdoors since 2010 during routine exotic mosquito species surveillance at companies that import used tires. To investigate the potential risk of establishment and spread of this invasive species at these locations, habitat suitability models were developed in Chapter 9. Two methodologies were used: first, a species distribution model based on the maximum entropy niche modelling approach (MAXENT) taking into consideration the most updated occurrence data of the species in Europe, and second, a spatial logic conditional model based on the temperature requirements of the species 
and using Land Surface Temperature data (LST-model). Results show differences in predicted habitat suitability values and patterns. The results of the MAXENT model are more restrictive for the species, the winter temperatures being the variable that contributed most to the performance of the model. The results of the LST-model show that 1) coastal areas are suitable for overwintering of eggs, 2) large areas in the northern part of the country have a low suitability for adult survival, and 3) the entire country is suitable for successful completion of the life cycle if the species is introduced after the winter months. Results of the LST-model reveals that temperatures in 2012 and 2014 did not limit the overwintering of eggs or survival of adults at the used tire companies where the species was found. By contrast, in 2010, 2011 and 2013, overwintering of eggs at these locations is not considered likely.

Finally, the main findings are discussed and placed into perspective in Chapter 10, by evaluating the factors that influence the acquired knowledge about the spatiotemporal distribution of mosquito species. Nationwide active surveillance of mosquitoes is pivotal to gain detailed information on the spatio-temporal distribution and abundance of mosquito species in the country. The geospatial occurrence data obtained through this surveillance have also shown useful for studying habitat suitability for populations of An. plumbeus, a potential malaria vector whose occurrence strongly correlates to environmental variables. Furthermore, this thesis highlighted the main pathways for introduction and dispersal of IMS taking into account current knowledge and expert opinion. The risk-based and adaptive surveillance of IMS described in my study revealed the annual introductions of Ae. albopictus since 2010 at used tire companies and Lucky bamboo greenhouses, sporadic findings of Ae. japonicus associated with used tire trade, the first aircraft associated import of Ae. aegypti in Europe, and the first associated import of Ae. japonicus with Lucky bamboo plants elsewhere in the world. The control of these IMS implemented after detection has proven effective to avoid proliferation at these locations and their immediate surroundings. Results of my research show that the current average climatic conditions limit the overwintering of eggs of $A e$. albopictus and their survival as adults in many areas of the country. However, due to the expected increase of temperature in the next decades as a result of climate change, several parts of the Netherlands may offer climatic conditions that are suitable to sustain populations of this species. Due to the adaptability of Ae. albopictus to new environmental conditions, mosquito control actions applied to this IMS in order to eradicate early stage introductions are crucial for avoiding establishment of the species at more northern latitudes. 



\section{Acknowledgements}


The older you get, the more experience you accumulate, the more people you meet, and the more people you are grateful to for their contribution to your life. I'm above the average age of students finishing a $\mathrm{PhD}$, but instead of presenting you with a very long acknowledgement section, you are probably facing one of the shortest acknowledgements ever written in a thesis at Wageningen University. If you are trying to find your name in this section, please stop, you will not find it. If you know m e, you will know that I'm agrateful person, and I will have thanked you in person for your help or collaboration, in the thesis Chapters or as coauthorship. If you are my family or one of my friends, I don't need to include you here because you already know how much I love you and I thank you every day for being in my life. However, due to their support in realizing this thesis, I especially thank the Netherlands Food and Consumer Product Safety Authority (NVWA), my promotor and co-promotors, and my dear National Reference Centre (NRC) and One Health Entomology (OHE) colleagues.

The only name that will be mentioned in this acknowledgements section is Ernst-Jan Scholte. In fact, this complete thesis is dedicated to him. This complete book is a legacy of his vision and work at the Centre for Monitoring of Vectors. During the writing of the proposal, the design of the experiments, the field work, the analysis of the results, and all other activities, I was guided by the answer to the question "What would Ernst-Jan advise me?". I always imagined him smiling, and with clear instructions, helping me through the most difficult issues. I admired him as a colleague and a friend, and I see Ernst-Jan as an example to follow in personal and professional matters. Wherever you are my friend, you are still in our minds. We won't forget you.

"Colui il cui nome è sempre pronunciato resta in vita” (G. Rodari) 



\section{Curriculum vitae}




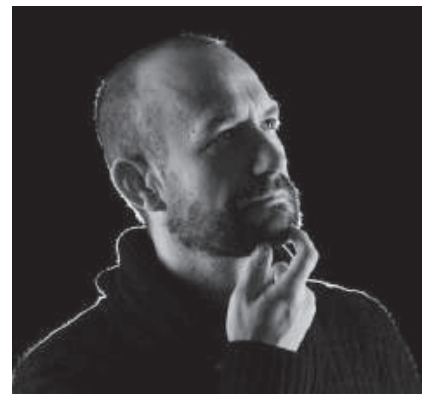

Adolfo Ibáñez-Justicia was born on 30 October 1976 in Valencia (Spain). After attending secondary school at Benlliure high school in Valencia, he started his bachelor degree studies in Forest Engineering at the Polytechnic University of Valencia, followed by a master degree at the University of Lleida. During his MSc thesis performed in 2000 in collaboration with the École d'Ingénieurs de PURPAN (Toulouse, France), he developed an interest in entomology and trapping methods for collecting insects. In 2001 he started to work at PYG Estructuras Ambientales SL. (Valencia, Spain). He was in charge of the monitoring of forest pests, the planning and implementation of integrated forest pests control, and the identification of insects. Between 2003 and 2005 he also followed a post-graduate degree as specialist on agricultural phytopathology and entomology at Polytechnic University of Valencia. In 2006 he started to work in the laboratory of forest entomology in the Centre per a la Investigació i Experimentació Forestal (CIEF, Valencia). He was then in charge of the morphological identification of insects, planning of monitoring and control of forest pests, and analysis and publication of the results. At the end of 2007, motivated in finding new challenges, he moved to the Netherlands. In 2010, after an adaptation period, learning the Dutch language, some temporary jobs, and building a professional network, he started to work as entomologist at the National Reference Centre (NRC) of the Netherlands Food and Consumer Safety Authority (NVWA). In this position he worked on contingency plans, he conducted quick scans for new introduced insects, and he worked on pest risk assessments as for example for the longhorn beetle Apriona sp. and the Asian hornet Vespa velutina nigrithorax. For these species he also studied the suitability of the climate of the Netherlands using models. In 2011 he started to work as researcher at the recently founded Centre for Monitoring of Vectors (CMV) of the NVWA. Since then he work collecting, analyzing and publishing data on vector species, making habitat suitability models, and advising on monitoring and control actions against invasive mosquito species. In 2015 he started his $\mathrm{PhD}$ at Wageningen University \& Research under the supervision of prof. Willem Takken and dr. Sander Koenraadt of the Laboratory of Entomology, and dr. Ron van Lammeren of the Laboratory of Geo-information Science and Remote Sensing. Within this project he developed methodologies for knowledge acquisition of the actual and potential distribution of indigenous and exotic mosquito species in the Netherlands. Furthermore, his thesis highlighted the main pathways for introduction and dispersal of invasive mosquito species (IMS), designed a risk-based surveillance of IMS, and evaluated the surveillance and control measures applied in the Netherlands against IMS introductions. Because in the near future, new challenges are expected in vector research, and introduction of invasive mosquito species is expected in the Netherlands, after finishing his $\mathrm{PhD}$, Adolfo aims to help the CMV and the Dutch authorities collecting, analyzing and disseminating data on vectors of diseases. 



\section{List of publications}




\section{Published papers}

Ibañez-Justicia, A., Poortvliet, P.M., Koenraadt, C.J.M. 2018. Evaluating perceptions of risk in mosquito experts and identifying undocumented pathways for the introduction of invasive mosquito species into Europe. Medical and Veterinary Entomology: MVE12344, DOI: $10.1111 /$ mve.12344. (Chapter 5 in this thesis)

Ibañez-Justicia, A., Teekema, S., den Hartog, W., Jacobs, F., Dik, M., Stroo, A. 2018. The effectiveness of Asian Bush Mosquito (Aedes japonicus japonicus) control actions in colonised peri-urban areas in the Netherlands. Journal of Medical Entomology 55(3):673-680. (Chapter 8 in this thesis)

Ibañez-Justicia, A., Gloria-Soria, A., den Hartog, W., Dik, M., Jacobs, F., Stroo, A. 2017. The first detected airline introductions of yellow fever mosquitoes (Aedes aegypti) to Europe, at Schiphol International airport, the Netherlands. Parasites\&Vectors 10:603. (Chapter 7 in this thesis)

van de Vossenberg, B.T., Ibañez-Justicia, A., Metz-Verschure, E., van Veen, E.J., BruilDieters, M.L., Scholte, E.J. 2015. Real-time PCR tests in Dutch exotic mosquito surveys; optimization of Aedes aegypti and Aedes albopictus identification tests, and the development of tests for the identification of Aedes atropalpus and Aedes japonicus japonicus (Diptera: Culicidae). Journal of Medical Entomology 52:336350.

Ibañez-Justicia, A., Cianci, D. 2015. Modelling the spatial distribution of the nuisance mosquito species Anopheles plumbeus (Diptera: Culicidae) in the Netherlands. Parasites\&Vectors 8:258 (Chapter 3 in this thesis)

Cianci, D., Hartemink, N., Ibañez-Justicia A. 2015. Modelling the potential spatial distribution of mosquito species in the Netherlands using three different modelling techniques. International Journal of Health Geographics 14:10

Vogels, C.B., van de Peppel, L.J., van Vliet, A.J., Westenberg, M., Ibañez-Justicia, A., Stroo, A., Buijs, J.A., Visser, T.M., Koenraadt, C.J. 2015. Winter Activity and Aboveground Hybridization Between the Two Biotypes of the West Nile Virus Vector Culex pipiens. Vector Borne Zoonotic Diseases 15:619-626

Zielke, D.E., Ibañez-Justicia, A., Kalan, K., Merdić, E., Kam pen, H., Werner, D. 2015. Recently discovered Aedes japonicus japonicus (Diptera: Culicidae) populations in The Netherlands and northern Germany resulted from a new introduction event and from a split from an existing population. Parasites\&Vectors 8:40

Ibañez-Justicia, A., Stroo, A., Dik, M., Beeuwkes, J., Scholte, E.J. 2015. National Mosquito (Diptera, Culicidae) Survey in the Netherlands 2010-2013. Journal of Medical Entomology 52:185-198 (Chapter 2 in this thesis)

Swart, A., Ibañez-Justicia, A., Buijs, J., van Wieren, S.E., Hofmeester, T.R., Sprong, H., Takumi, K. 2014. Predicting tick presence by environmental risk mapping. Frontiers in Public Health 2:238.

Scholte, E.J., Ibañez-Justicia, A., Stroo, A., De Zeeuw, J., den Hartog, W., Reusken, C. 2014. Mosquito collections on incoming intercontinental flights at Schiphol International airport, the Netherlands, 2010-2011. Journal of the European Mosquito Control Association 32: 17-21.

Ibañez-Justicia, A., Kampen, H., Braks, M., Schaffner, F., Steeghs, M., Werner, D., Zielke, D., den Hartog, W., Brooks, M., Dik, M., van de Vossenberg, B., Scholte, E.J. 2014. 
First report of Aedes japonicus japonicus (Theobald, 1901) (Diptera, Culicidae) population in The Netherlands. Journal of the European Mosquito Control Association. 32:9-13.

Koenraadt, C.J., Balenghien, T., Carpenter, S., Ducheyne, E., Elbers, A.R., Fife, M., Garros, C., Ibañez-Justicia, A., Kampen, H., Kormelink, R.J., Losson, B., van der Poel, W.H., De Regge, N., van Rijn, P.A., Sanders, C., Schaffner, F., Sloet van Oldruitenborgh-Oosterbaan, M.M., Takken, W., Werner, D., Seelig, F. 2014. Buetongue, Schmallenberg - what is next? Culicoides-borne viral diseases in the 21st Century. BMC Veterinary Research 10:77.

Scholte, E.J., Mars, M.H., Braks, M., den Hartog, W., Ibañez-Justicia, A., Koopmans, M., Koenraadt, C.J., De Vries, A., Reusken, C. 2012. No evidence for the persistence of Schmallenberg virus in overwintering mosquitoes (Diptera: Culicidae) in the Netherlands. Medical and Veterinary Entomology. 28:110-115.

Scholte, E.J., Dik, M., Ibañez-Justicia, A., den Hartog, W., Schoelitsz, B., Brooks, M., Braks, M., Steeghs, M. 2012. Findings and control of two invasive exotic mosquito species, Aedes albopictus and Ae. atropalpus (Diptera: Culicidae) in the Netherlands, 2011. Journal of the European Mosquito Control Association 30: 1-14.

Ibañez-Justicia, A., Soto, A., Martínez Gonzalvo, M., Pérez-Laorga Arias, E. 2008. Análisis de las defoliaciones y muestreo con feronoma sexual de Lymantria dispar (L.) (Lepidoptera: Lymantriidae) en las principales masas de Quercus de la Comunitat Valenciana. Años 2000-2006. Cuadernos de la Sociedad Española de Ciencias Forestales 26: 73-78.

Ibañez-Justicia, A., Martínez Gonzalvo, M., Pérez-Laorga Arias, E., Soto, A. 2007. Distribución y abundancia de Lymantria dispar (Linnaeus, 1758) (Lepidoptera: Lymantriidae) en las principales masas de carrasca Quercus ilex (L.) subsp. rotundifolia (Lam.) y alcornoque Quercus suber (L.) de la Comunitat Valenciana. Boletín de sanidad vegetal - Plagas 33: 491-502.

\section{Submitted papers}

Ibañez-Justicia, A., van Lammeren, R., Koenraadt, C.J.M., Bergsma, A., Delucchi, L., Rizzoli, A., Takken, W. Habitat suitability modelling to assess the introductions of Aedes albopictus (Diptera, Culicidae) in the Netherlands. Submitted. (Chapter 9 in this thesis)

\section{Other publications}

Stroo, A., Ibañez-Justicia, A., Braks, M. 2018. Towards a policy decision on Aedes japonicus - Risk assessment of Aedes japonicus in the Netherlands. RIVM Letter report 2018-0091. DOI 10.21945/RIVM-2018-0091

Ibañez-Justicia, A., Dik, M., den Hartog, W., van den Biggelaar, R., Stroo, A. 2014. Aedes j. japonicus mosquito surveillance in the province of Flevoland (The Netherlands). Proceedings of the Netherlands Entomological Society Meeting, volume 26: 33-42.

Ibañez-Justicia, A., Scholte, E.J., Reusken, C., de Vries, A., Dik, M., Braks, M. 2011. A new, additional approach for arbovirus surveillance in The Netherlands: the use of 
honey-baited cards to detect circulating mosquito-borne viruses. Proceedings of the Netherlands Entomological Society Meeting, volume 23: 9-17

Ibañez-Justicia, A., Loomans, A. 2011. Mapping the potential occurrence of an invasive species by using CLIMEX: case of the Asian hornet (Vespa velutina nigrithorax) in The Netherlands. Proceedings of the Netherlands Entomological Society Meeting, volume 22: 39-46.

Ibañez-Justicia, A., Potting, R. 2010. Pest Risk Assessment Apriona species (A. germari, A. japonica and $A$. cinerea). NPPO of The Netherlands, Wageningen (NL), 27 pp. 

Training and education statement 


\section{PE\&RC Training and Education Statement}

With the training and education activities listed below the $\mathrm{PhD}$ candidate has complied with the requirements set by the C.T. de Wit Graduate School for Production Ecology and Resource Conservation (PE\&RC) which comprises of a minimum total of 32 ECTS ( $=22$ weeks of activities)

\section{Review of literature (6 ECTS)}

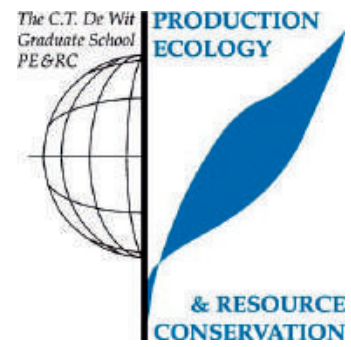

- An updated and comprehensive review of the pathways for introduction and spread of invasive mosquito species in Europe

\section{Writing of project proposal (4.5 ECTS)}

- Geospatial modelling of mosquito borne diseases risk in the Netherlands

\section{Post-graduate courses (12.4 ECTS)}

- Second FP7 VECTORIE training course: anti-vector, anti-virus, pro-one health: tackling emerging viral vector-borne diseases in Europe; WUR, Wageningen, NL (2014)

- International SBV/Culicoides workshop; WUR, Wageningen, NL (2013)

- Vector-borne surveillance projects VICE project, CoVetLab project workshop; VICE/CoVetLab, Schiphol, NL (2013)

- Pest risk assessment workshop; EPPO/PRATTIQUE, Hammamet, TN (2010)

- Workshop CLIMEX v3 training; EPPO, Angers, FR (2010)

- Series of courses on plant production and agro-forestry ecosystems: specialization plant health (university degree); Polytechnic University of Valencia, ES (2003-2005)

Laboratory training and working visits (5.4 ECTS)

- Modeling spatial distribution mosquitoes; Faculty of Veterinary, Medicine, Utrecht University, NL (2014-2015)

- VecMap Workshop for project users; AVIA-GIS, Antwerp, BE (2013)

- Mosquito species collection; Naturalis, Leiden, NL (2013)

- Training in risk mapping using eRiskMapper; ERGO Ltd., Oxford University, UK (2012)

- Vector competence Rift Valley fever collaboration; Central Veterinary Institute, Lelystad, NL (2011)

\section{Invited review of (unpublished) journal manuscript (5 ECTS)}

- Frontiers in Microbiology, section Infectious Diseases: modelling for determining areas with the possibility of presence of dengue and zika vector Aedes albopictus, across Iran's borders with Pakistan (2017)

- Acta tropica: the invasive Asian tiger mosquito Aedes albopictus (Diptera: Culicidae) in Germany: local reproduction and overwintering raise concern for soon establishment (2016)

- Parasites \& Vectors: modelling spatial distribution vectors of RVF Senegal (2015)

- Medical and Veterinary Entomology: Aedes japonicus traps evaluation Switzerland (2015)

- Parasites \& Vectors: West Nile virus surveillance in the North Kent marshes, UK (2014)

\section{Deficiency, Refresh, Brush-up courses (7.8 ECTS)}

- VecMap on tour; AVIA-GIS, WUR, Wageningen, NL (2017)

- EndNote course; NVWA, WUR, Wageningen, NL (2017)

- Training in sampling and identification ticks; E-SOVE/EMCA, Lisbon, PT (2016)

- Training identification European Culicoides; NVWA-Academie, Wageningen, NL (2016)

- Cursus toegepaste statistiek; WUR, Wageningen, NL (2015)

- Training identification European mosquitoes; AVIA-GIS, Wageningen, NL (2015) 
- Training in sampling and identification sandflies; E-SOVE/EMCA, Thessaloniki, GR (2014)

- Course Arc-GIS spatial analyst; Nieuwland, Wageningen, NL (2012)

- Training identification European mosquitoes; AVIA-GIS, Brussels, BE (2011)

Competence strengthening / skills courses (5.1 ECTS)

- Research integrity; WUR, Wageningen, NL (2017)

- Didactics and communication skills for ELE teachers; Cervantes Institute \& University of Valencia, ES (2006)

- Course of pedagogical aptitude; Instituto Ciencias de la Educación, Valencia, ES (2001-2002)

PE\&RC Annual meetings, seminars and the PE\&RC weekend (1.5 ECTS)

- PE\&RC Last years weekend (2018)

- PE\&RC First years weekend (2017)

\section{Discussion groups / local seminars / other scientific meetings (7.5 ECTS)}

- Copernicus European health workshop (2018)

- VectorNet IT meeting; ECDC-EFSA, Antwerp, Belgium (2017)

- MEMO Project supervisory committee; Brussel, Belgium (2017)

- Vector / one health group; WUR (2016-2018)

- Start symposium molecular and evolutionary ecology course; WUR (2016)

- $\quad$ Dutch ARboviral Network (DARN) workshop; WUR (2016)

- Vector modelling meetings (VBD and vectors); RIVM \& University of Utrecht (20142015)

- Dutch symposium on emerging Bunyaviruses; Central Veterinary Institute (CVI) (2014)

- Kenniskring medische milieukunde; GGD\&RIVM, Bilhoven (2013)

- $\quad$ Ticks monitoring results; WUR (2013)

- $\quad$ Netherlands Entomological Society day (2010-2015)

- 10 th IUFRO Working party 7.03.10, workshop on biotic risks and climate change in forests; Freiburg, Germany (2010)

\section{International symposia, workshops and conferences (20.8 ECTS)}

- European Mosquito Control Association workshop; Speyer, Germany; oral presentation (2018)

- $7^{\text {th }}$ International Congress of the Society for Vector Ecology; Palma de Mallorca, Spain; poster presentation (2017)

- VIII ${ }^{\text {th }}$ European Mosquito Control Association conference; Becici, Montenegro; oral presentation (2017)

- $20^{\text {th }}$ European Society of Vector Ecology conference, Lisbon, Portugal; oral presentation (2016)

- VII ${ }^{\text {th }}$ European Mosquito Control Association conference; Valencia, Spain; oral presentation (2015)

- Vector-Borne Diseases in the UK Biennial Conference; Liverpool, England; oral presentation (2014)

- $19^{\text {th }}$ European Society of Vector Ecology conference, Thessaloniki, Greece; poster presentation and oral presentation (2014)

- The Life Conops project: systems for the surveillance of invasive mosquito species workshop; Bologna, Italy; oral presentation (2014)

- Vbornet-EMCA joint meeting; Antwerp, Belgium; oral presentation (2013)

- European Mosquito Control Association workshop; Speyer, Germany; oral presentation (2013)

- $\quad 18^{\text {th }}$ European Society of Vector Ecology conference; Montpellier, France (2012)

- WHO/EMCA Conference on vector-related risk of introduction of Chikungunya and Dengue fever and spread of Ae. albopictus and Ae. japonicus within Europe; Speyer, Germany (2011) 
Thesis lay-out: Adolfo Ibáñez-Justicia

Cover design: Elfi Voermans (http://www.11i.nl)

Photograph back cover: Pau Ferrer i Poblete

Printed by Digiforce $\|$ ProefschriftMaken 



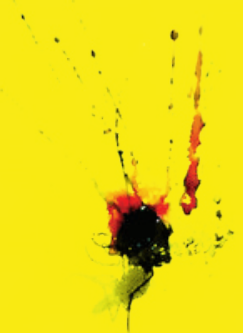

